Aus der Klinik für Kardiologie und Pneumologie

(Prof. Dr. med. G. Hasenfuß)

der Medizinischen Fakultät der Universität Göttingen

\title{
Akuter ST-Strecken-Hebungsinfarkt \\ (STEMI) bei Patientinnen und Patienten \\ mit zusätzlich chronischem Verschluss \\ in einem Nicht-Infarkt-Gefäß: klinische \\ Relevanz und prognostische Bedeutung
}

\author{
INAUGURAL-DISSERTATION \\ zur Erlangung des Doktorgrades \\ der Medizinischen Fakultät der \\ Georg-August-Universität zu Göttingen
}

vorgelegt von

Matthias Scholz

aus

Hildesheim

Göttingen 2017 
Dekan:

Referent:

Ko-Referentin:
Prof. Dr. rer. nat. H. K. Kroemer

Prof. Dr. L. S. Maier

PD Dr. H. Schneider

Datum der mündlichen Prüfung: 24.04.2018 
Hiermit erkläre ich, die Dissertation mit dem Titel "Akuter STStrecken-Hebungsinfarkt (STEMI) bei Patientinnen und Patienten mit zusätzlich chronischem Verschluss in einem Nicht-Infarkt-Gefäß: klinische Relevanz und prognostische Bedeutung" eigenständig angefertigt und keine anderen als die von mir angegebenen Quellen und Hilfsmittel verwendet zu haben.

Göttingen, den 


\section{Inhaltsverzeichnis}

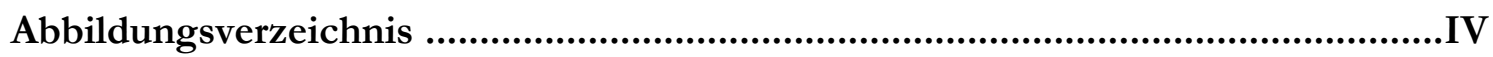

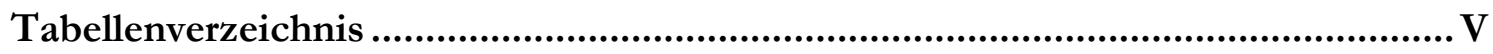

Abkürzungsverzeichnis....................................................................................... VII

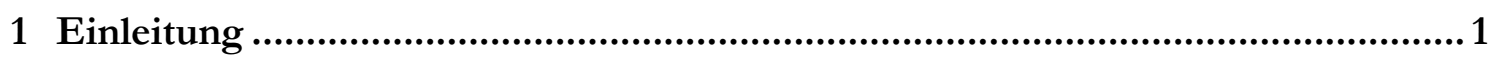

1.1 Epidemiologie der koronaren Herzkrankheit............................................................................

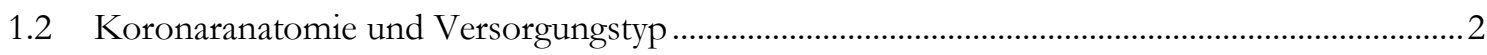

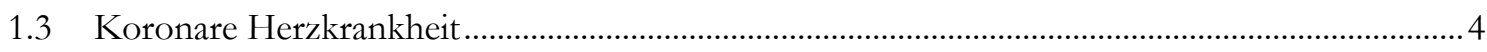

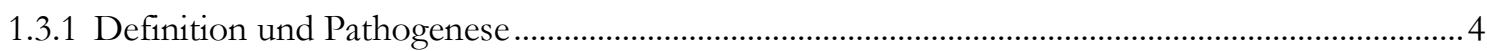

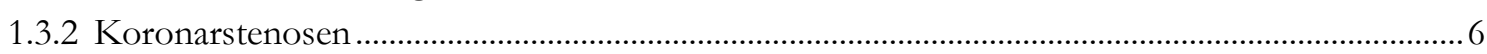

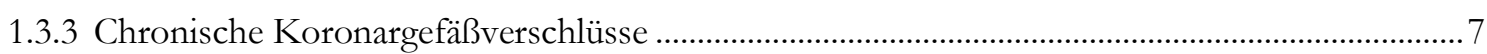

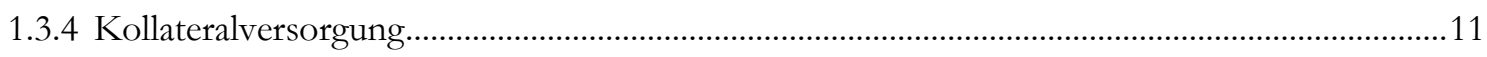

1.4 Akutes Koronarsyndrom und ST-Strecken-Hebungsinfarkt ......................................................13

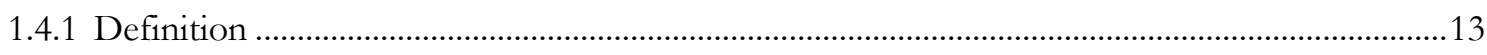

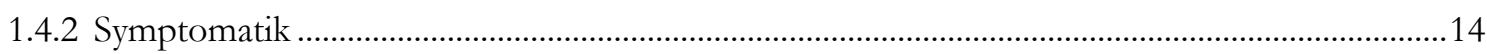

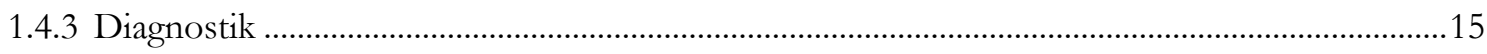

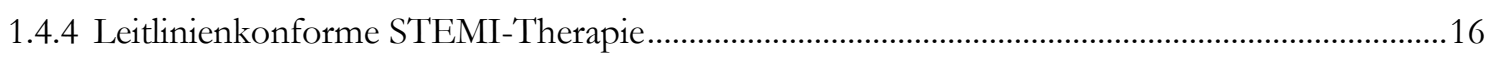

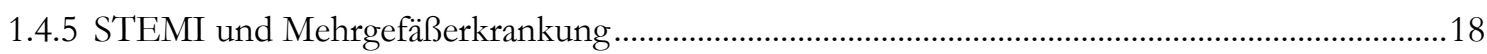

1.4.6 STEMI mit zusätzlich chronisch verschlossenem Nicht-Infarkt-Gefäß....................................18

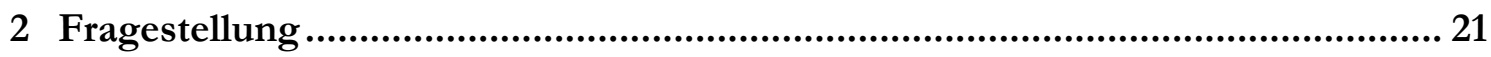

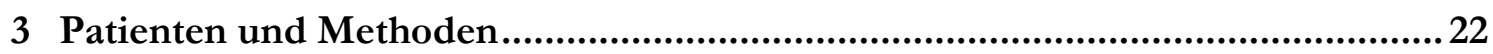

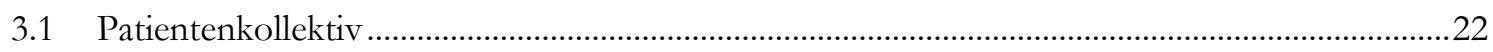

3.2 Aus der Analyse ausgeschlossene Patienten ...........................................................................22

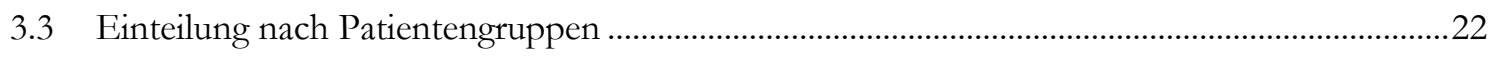

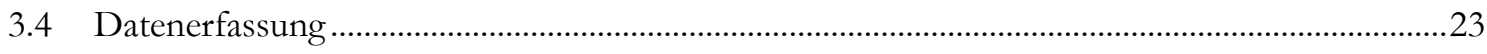

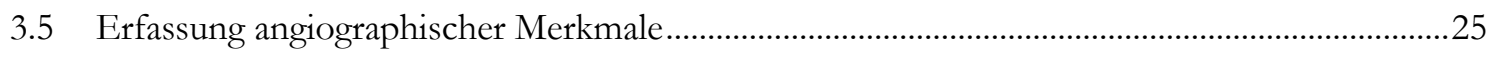

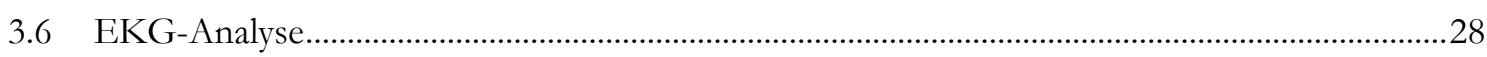

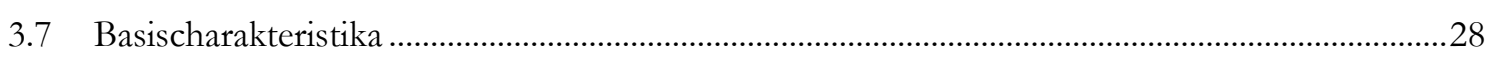

3.8 Behandlungszeiten und rettungstechnische Daten .....................................................................

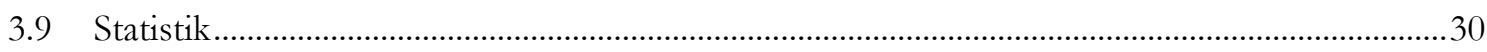

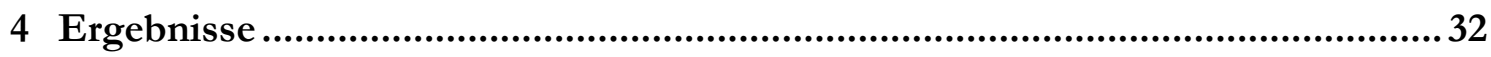

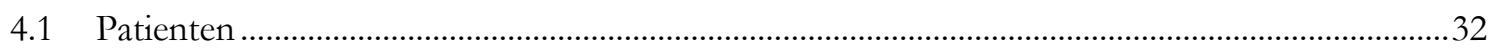

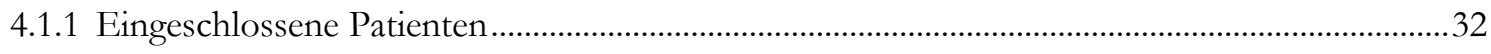

4.1.2 Transportart und Patientenherkunft ........................................................................................... 34

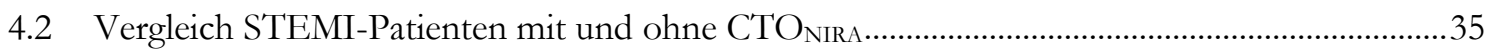

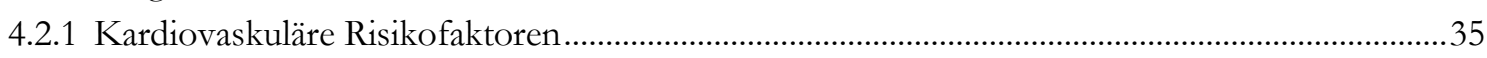

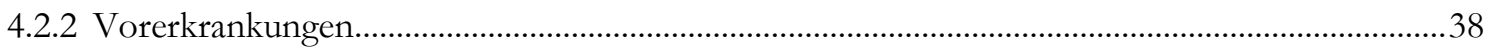

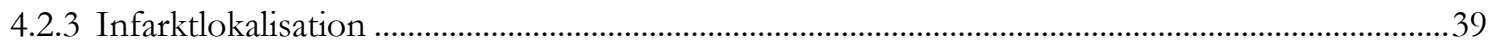


4.2.4 LV-Funktion

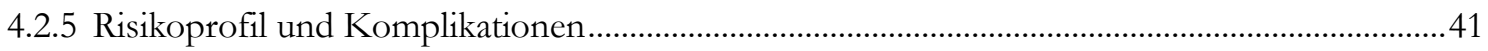

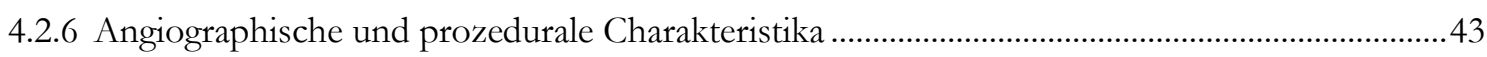

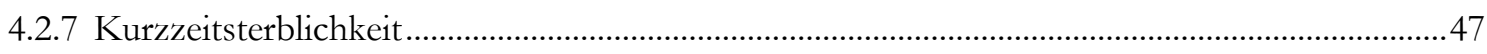

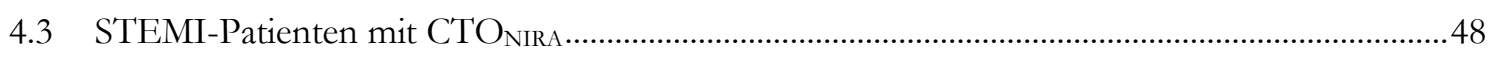

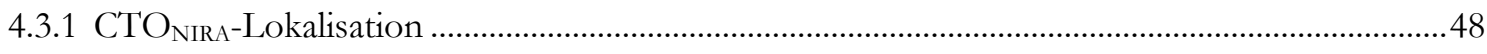

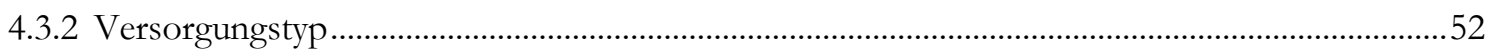

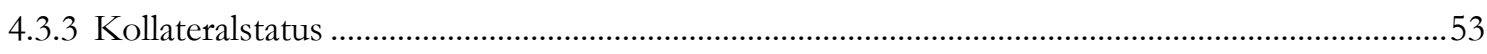

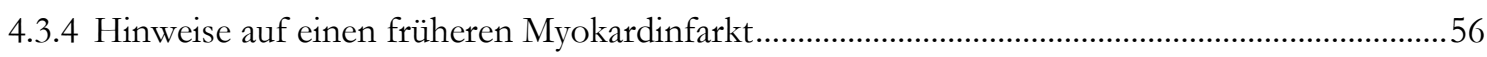

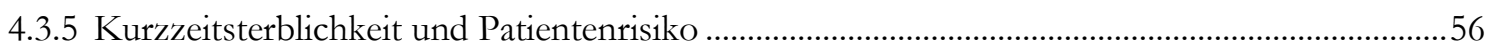

4.3.5.1 Kurzzeitsterblichkeit und TIMI Risk Score abhängig vom betroffenen CTO-Gefäß ............57

4.3.5.2 Kurzzeitsterblichkeit abhängig vom akut betroffenen STEMI-Gefäß …………………….......58

4.3.5.3 Kurzzeitsterblichkeit unter Berücksichtigung von CTO $_{\text {NIRA }}$-und STEMIGefäßlokalisation

4.3.5.4 Kurzzeitsterblichkeit und TIMI Risk Score abhängig vom CTO-Gefäßabschnitt.

4.3.5.5 Kurzzeitsterblichkeit abhängig von der CTO-Segmentlokalisation und dem Koronarversorgungstyp.

4.3.5.6 Kurzzeitsterblichkeit abhängig von der Kollateralversorgung.

4.3.5.7 Kurzzeitsterblichkeit bei Patienten mit und ohne Hinweis auf einen früheren Myokardinfarkt.....

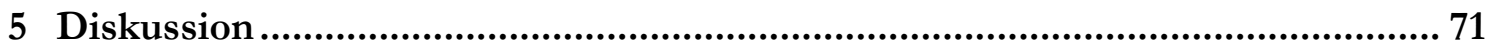

5.1 STEMI-Patienten mit und ohne zusätzlich chronischen Verschluss.............................................72

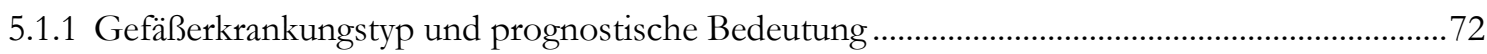

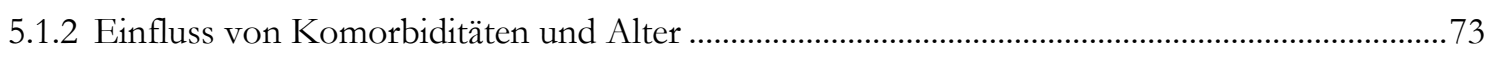

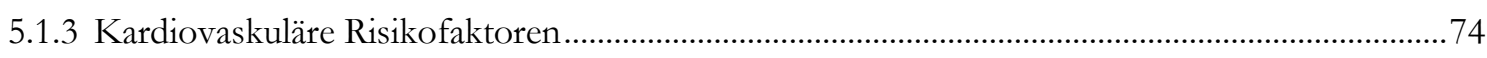

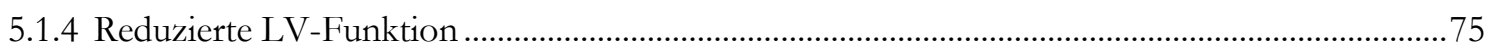

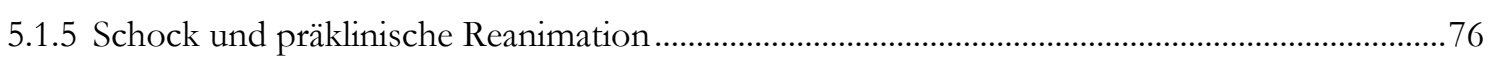

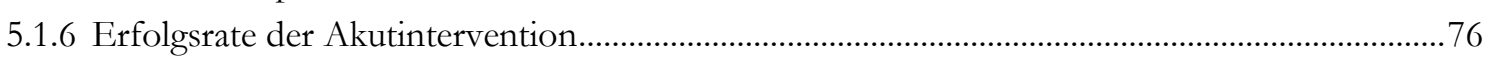

5.2 STEMI-Patienten mit zusätzlich chronischem Verschluss: Bedeutung von

Koronarstatus, Kollateralversorgung und früherem Infarkt ......................................................77

5.2.1 Vorkommen eines CTO NIRA $_{\text {in }}$ den unterschiedlichen Koronargefäßen.......................................77

5.2.2 Prognostische Bedeutung der Lokalisation des CTO $_{\text {NIRA }}$ in den unterschiedlichen Koronargefäßen

5.2.3 Prognostische Bedeutung der Lokalisation des CTO $_{\text {NIRA }}$ in unterschiedlichen Koronargefäßsegmenten.

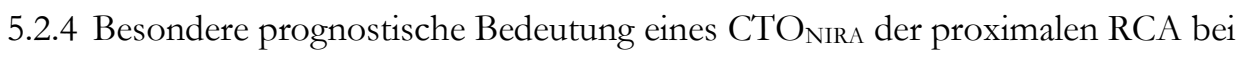

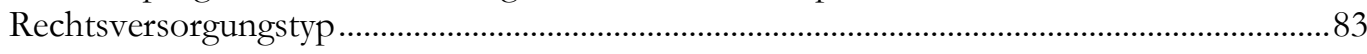

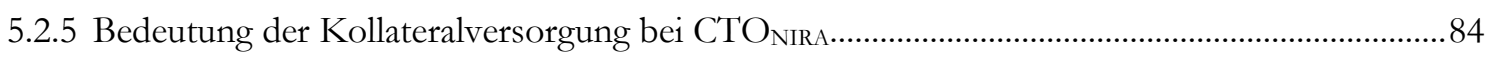

5.2.6 Besondere Bedeutung einer Kollateralisierung über das Infarktgefäß als Donor-Arterie..........85

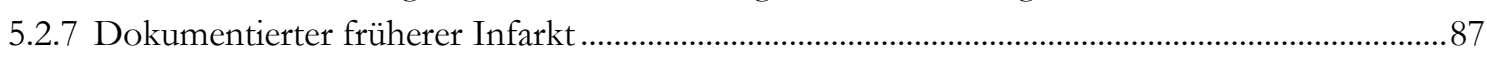

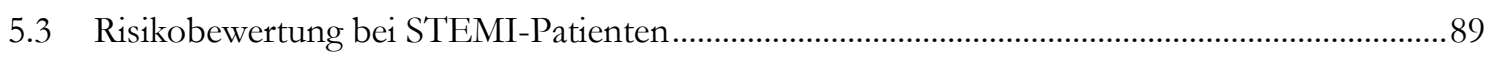

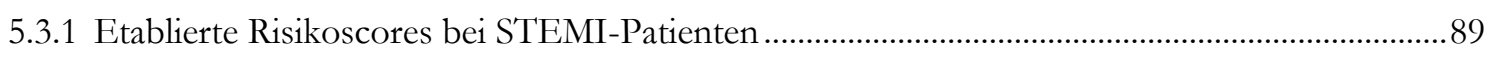

5.3.2 Risikobewertung im Rahmen der Qualitätssicherung ……………............................................ 91

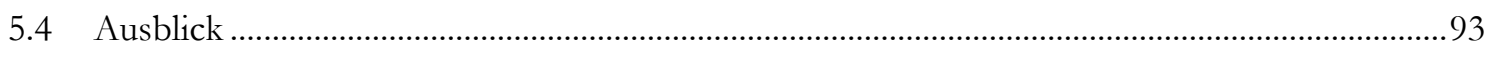

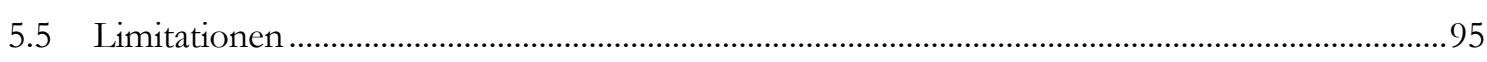

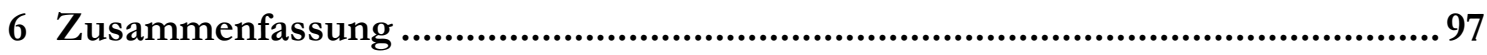




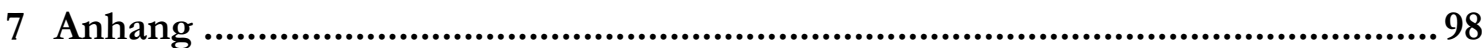

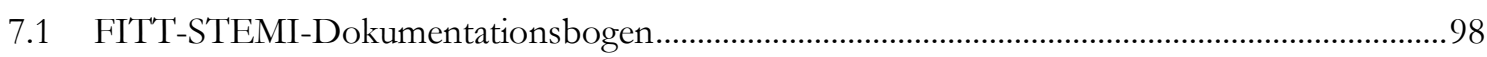

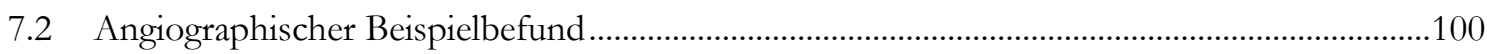

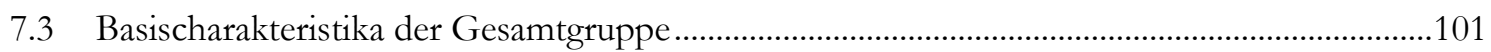

7.4 Basischarakteristika nach CTO-Gefäßlokalisation...................................................................102

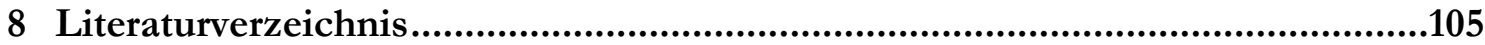




\section{Abbildungsverzeichnis}

Abbildung 1: Segmentdefinition nach: The SYNTAX Score (Sianos et al. 2005) ..................................27

Abbildung 2: Flussdiagramm Patienteneinschluss ................................................................................

Abbildung 3: Anteil des Gefäßerkrankungstyps der Patienten in \%....................................................33

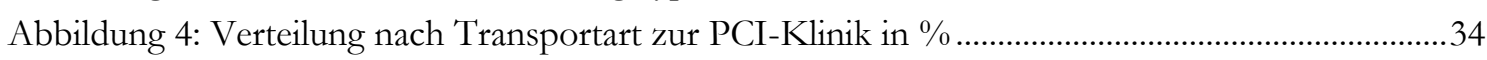

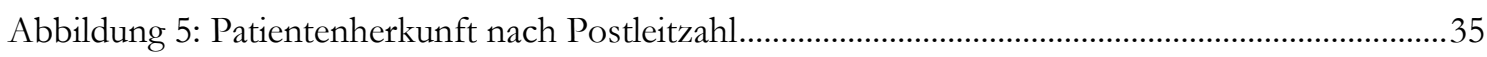

Abbildung 6: Geschlechterverteilung in der Gesamtgruppe und bei Ein- und Mehrgefäß-KHK

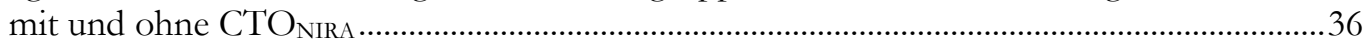

Abbildung 7: Durchschnittliches Patientenalter in der Gesamtgruppe und bei Ein- und Mehrgefäß-KHK mit und ohne CTO

Abbildung 8: Anteil der Patienten mit Diabetes mellitus in der Gesamtgruppe und bei Ein-

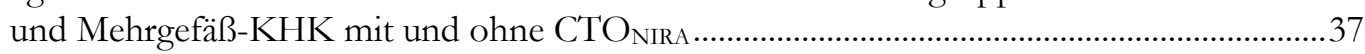

Abbildung 9: Anteil der Raucher in der Gesamtgruppe und bei Ein- und Mehrgefäß-KHK mit

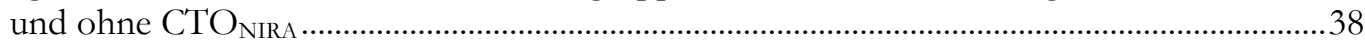

Abbildung 10: Anteil Patienten mit Myokardinfarkt in der Vorgeschichte in der Gesamtgruppe und bei Ein- und Mehrgefäß-KHK mit und ohne CTO

Abbildung 11: Anteil Patienten mit Apoplex in der Vorgeschichte in der Gesamtgruppe und

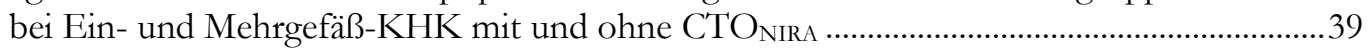

Abbildung 12: Infarktlokalisation im EKG

Abbildung 13: Anteil der LV-Ejektionsfraktionsbereiche in der Gesamtgruppe und bei Einund Mehrgefäß-KHK mit und ohne CTO

Abbildung 14: Anteil TIMI Risk Score-Gruppen in der Gesamtgruppe und bei Ein- und Mehrgefäß-KHK mit und ohne CTONIRA

Abbildung 15: Anteil Killip-Klassifikation in der Gesamtgruppe und bei Ein- und MehrgefäßKHK mit und ohne CTO $\mathrm{NIRA}_{\text {. }}$

Abbildung 16: Anteil der Patienten mit präklinischer mechanischer Reanimation in der Gesamtgruppe und bei Ein- und Mehrgefäß-KHK mit und ohne CTO $\mathrm{O}_{\text {NIRA }} \ldots \ldots \ldots \ldots \ldots \ldots \ldots \ldots . . . . . . . . .43$

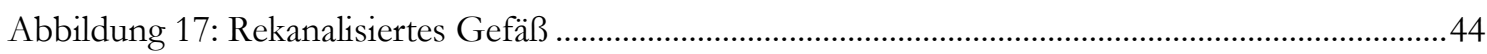

Abbildung 18: Anteil TIMI Grade Flow Vor-PCI in \% in der Gesamtgruppe und bei Ein- und Mehrgefäß-KHK mit und ohne CTO

Abbildung 19: Anteil TIMI Grade Flow Nach-PCI in \% in der Gesamtgruppe und bei Ein-

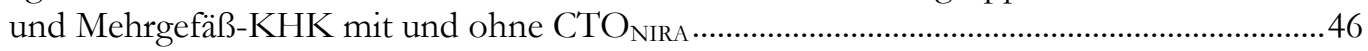

Abbildung 20: C2B-Zeiten im Median in der Gesamtgruppe und bei Ein- und Mehrgefäß-

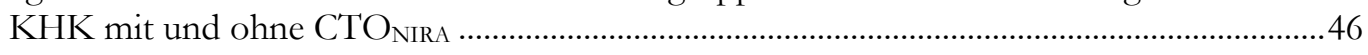

Abbildung 21: Krankenhaussterblichkeit in der Gesamtgruppe und bei Ein- und Mehrgefäß-

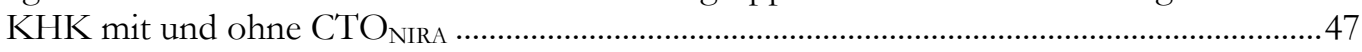

Abbildung 22: Krankenhaussterblichkeit bei Patienten mit Schock in der Gesamtgruppe und bei Ein- und Mehrgefäß-KHK mit und ohne CTO $_{\text {NIRA }}$..............................................................4

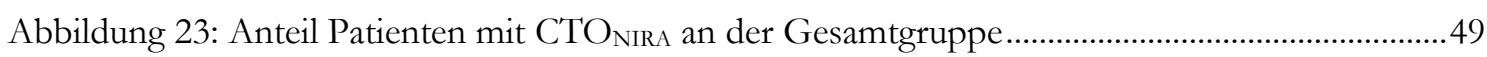

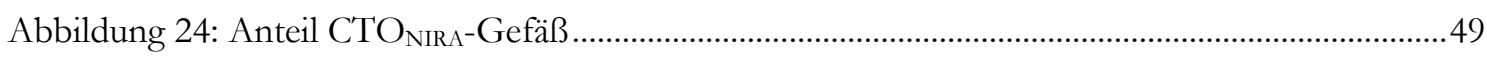

Abbildung 25: Anteil der CTO-Segmentlokalisation in der RCA ……………......................................50

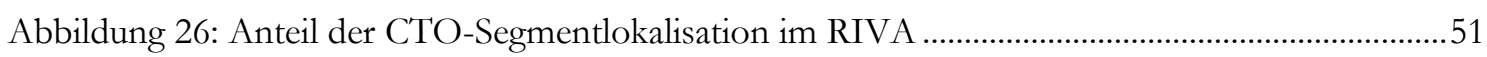

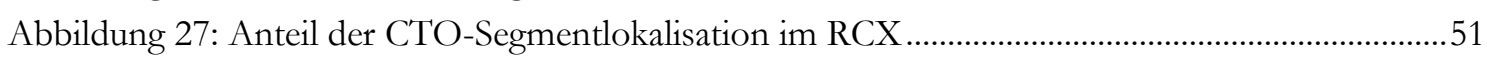

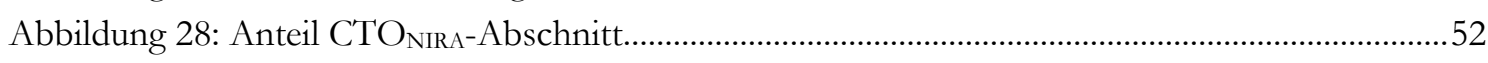

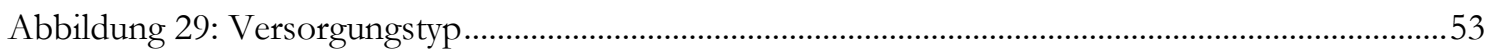

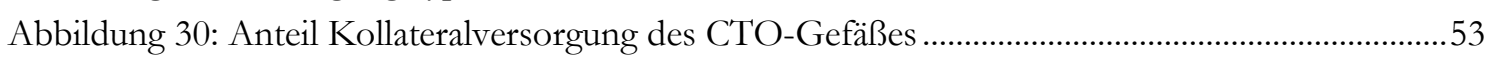

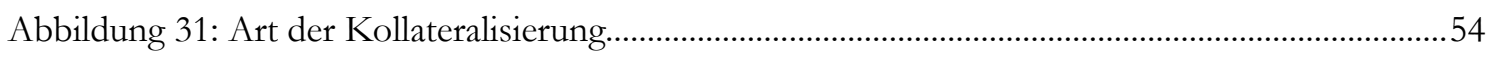

Abbildung 32: Herkunft der interkoronaren Kollaterale .....................................................................

Abbildung 33: Kollateralabgang in Bezug zum akuten Verschluss ......................................................55 
Abbildung 34: Krankenhaussterblichkeit (\%) in Abhängigkeit vom CTO-Gefäß...................................57

Abbildung 35: Anteil der Patienten mit Schock (\%) in Abhängigkeit vom CTO-Gefäß ......................58

Abbildung 36: Krankenhaussterblichkeit (\%) in Abhängigkeit vom CTO-Gefäß bei Patienten mit Schock und 1 CTO NIRA $_{\text {. }}$

Abbildung 37: Krankenhaussterblichkeit (\%) in Abhängigkeit vom akuten Infarktgefäß (culprit lesion) bei STEMI-Patienten mit PCI und 1CTO

Abbildung 38: Krankenhaussterblichkeit (\%) in Abhängigkeit vom CTO-Gefäßabschnitt .

Abbildung 39: Anteil der Patienten mit Schock (\%) in Abhängigkeit vom CTO-Gefäßabschnitt....62

Abbildung 40: Krankenhaussterblichkeit (\%) nach CTO-Gefäßsegmenten in der RCA in Abhängigkeit vom Versorgungstyp.

Abbildung 41: Krankenhaussterblichkeit (\%) nach CTO-Gefäßsegmenten im RIVA in Abhängigkeit vom Versorgungstyp

Abbildung 42: Krankenhaussterblichkeit (\%) nach CTO-Gefäßsegmenten im RCX in Abhängigkeit vom Versorgungstyp.....................................................................................66

Abbildung 43: Krankenhaussterblichkeit (\%) in Abhängigkeit von der Kollateralversorgung...........67

Abbildung 44: Krankenhaussterblichkeit (\%) nach Herkunft der Kollaterale .......................................68

Abbildung 45: Krankenhaussterblichkeit (\%) nach Ursprungsort der IRA-Kollaterale .......................68

Abbildung 46: Aktueller FITT-STEMI-Dokumentationsbogen (Stand 10/2016, Seite 1/2; (Scholz et al. 2012)) .98

Abbildung 47: Aktueller FITT-STEMI-Dokumentationsbogen (Stand 10/2016, Seite 2/2; (Scholz et al. 2012))

Abbildung 48: CTO in Segment 1 der RCA mit Brückenkollateralen...................................................100

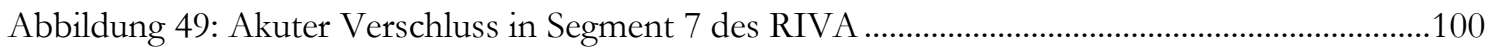

Abbildung 50: Akuter Verschluss in Segment 7 des RIVA nach Draht-Rekanalisation.....................100

Abbildung 51: Interkoronare Kollaterale via vormals verschlossenem RIVA nach

Rekanalisation mit Darstellung der peripheren RCA.

\section{Tabellenverzeichnis}

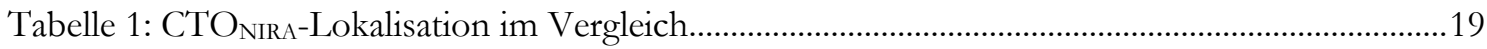

Tabelle 2: Im Dokumentationsbogen erfasste angiographische Merkmale ..........................................25

Tabelle 3: Im Dokumentationsbogen erfasste Patientencharakteristika..............................................29

Tabelle 4: Gründe für fehlende Angabe des Gefäßerkrankungstyps .......................................................

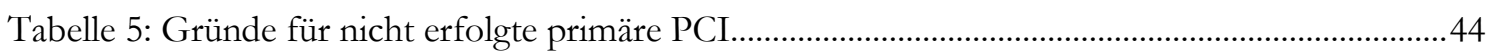

Tabelle 6: Anteil der Patienten mit Hinweis auf einen abgelaufenen, früheren Infarkt........................56

Tabelle 7: Zusammenhang zwischen CTO- und Infarktgefäß mit Blick auf das Patienten-

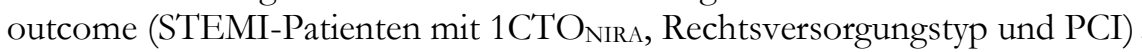

Tabelle 8: Zusammenhang zwischen CTO- und Infarktgefäß mit Blick auf das Patientenoutcome (STEMI-Patienten mit 1CTONIRA, Linksversorgungstyp und PCI)

Tabelle 9: Krankenhaussterblichkeit und unabhängige Prädiktoren bei STEMI-Patienten mit 1CTO ${ }_{\text {NIRA }}$; Multivariates Logit-Modell nach Vortest

Tabelle 10: Unabhängige Prädiktoren der Krankenhaussterblichkeit bei STEMI-Patienten mit 1 CTO $_{\text {NIRA; }}$ Multivariates Logit-Modell nach backward stepwise-Methode

Tabelle 11: Zusammenhang zwischen CTO- und Infarktgefäß mit Blick auf das Patienten-

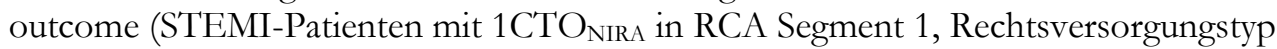
und PCI)

Tabelle 12: Kollateralisierung bei STEMI-Patienten mit 1CTO $\mathrm{NIRA}_{\text {in }}$ in $\mathrm{RCA}$.................................69

Tabelle 13: Kollateralisierung bei STEMI-Patienten mit 1CTO NIRA $_{\text {im RIVA }}$ 


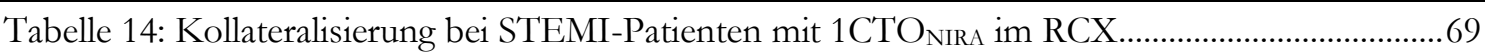

Tabelle 15: Krankenhaussterblichkeit bei Patienten mit und ohne dokumentierten früheren Infarkt

Tabelle 16: Basischarakteristika der Gesamtgruppe und bei Ein- und Mehrgefäß-KHK mit und ohne CTONIRA

Tabelle 17: Basischarakteristika aller STEMI-Patienten mit einem CTO $_{\text {NIRA }}$ in der Gesamtgruppe und nach CTO-Gefäßlokalisation

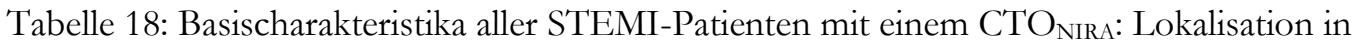
Segment 1 der RCA bei Rechtsversorgungstyp vs. alle anderen STEMI-Patienten mit einem CTO $\mathrm{NIRA}_{\mathrm{N}}$

Tabelle 19: Endgültiges Ergebnis der backward stepwise-Modell-Konstruktion nach 19 Schritten; Übersicht über alle in diesem multivariaten Modell berücksichtigten Variablen 


\section{Abkürzungsverzeichnis}

ACC

ACS

AHA

AP

BMI

C2B-Zeit

CABG

CC grade

CTO

CTO $_{\text {NIRA }}$

$\mathrm{DM}$

EKG

ESC

et al.

FITT-STEMI
American College of Cardiology

Acute coronary syndrome (akutes Koronarsyndrom)

American Heart Association

Angina Pectoris

Body Mass Index

Contact-to-ballon-Zeit

Coronary artery bypass graft (Koronararterienbypass)

Collateral connection grade (Kollateralverbindungs-Klassifizierung)

Chronic total occlusion (chronischer Totalverschluss)

Chronic total occlusion in a noninfarct-related artery (chronischer Totalverschluss in einem Nicht-Infarkt-Gefäß)

Diabetes mellitus

Elektrokardiogramm

European Society of Cardiology

et alii

Feedback-Intervention and Treatment-Times in ST-Elevation Myocardial

Infarction

$\mathrm{IIH}$

Infarkt Intrahospital

IRA

KHK

KHS

LCA

LMCA

LV

LVEF

MACCE

MVD

NSTEMI

PCI

PLA

RCA

RCX

RIVA

RIVP

SVD
Infarct-related artery (vom akuten Infarkt betroffene Koronararterie)

Koronare Herzkrankheit

Krankenhaus

Left coronary artery (linke Koronararterie)

Left main coronary artery (Hauptstamm der linken Koronararterie)

Linker Ventrikel

Linksventrikuläre Ejektionsfraktion

Major adverse cardiac and cerebrovascular events (schwere kardiale und zerebrovaskuläre Ereignisse: Schlaganfall, Herzinfarkt, Tod)

Multivessel disease (Mehrgefäßerkrankung)

Non ST-segment elevation myocardial infarction (Nicht-ST-Strecken-

Hebungsinfarkt)

Percutaneous coronary intervention (perkutane Koronarintervention)

Posterolateralast

Right coronary artery (rechte Koronararterie)

Ramus circumflexus

Ramus interventricularis anterior

Ramus interventricularis posterior

Singlevessel disease (Eingefäßerkrankung) 
STEMI ST-segment elevation myocardial infarction (ST-Strecken-Hebungsinfarkt)

TIMI

Thrombolysis in Myocardial Infarction

TRS TIMI Risk Score

UMG Universitätsmedizin Göttingen

WHO World Health Organization

ZVD Zerovessel disease (Nullgefäßerkrankung) 


\section{Einleitung}

\subsection{Epidemiologie der koronaren Herzkrankheit}

Ein Blick in die regelmäßig veröffentlichte Gesundheitsberichterstattung des Bundes genügt, um sich der Bedeutung der koronaren Herzkrankheit (KHK) für die Gesundheit in der Gesellschaft bewusst zu werden. Nach Angaben des Statistischen Bundesamtes und des Robert Koch-Institutes stehen in der Bundesrepublik Deutschland mit 39,7 \% aller Todesfälle auch weiterhin die Erkrankungen des Kreislaufsystems an der Spitze der Todesursachen. Sowohl bei den Frauen (13,3\% aller Todesfälle) als auch bei den Männern (15,6\% aller Todesfälle) führen die ischämischen Herzkrankheiten die Todesursachenstatistiken an. Das Hauptaugenmerk liegt dabei auf dem akuten Myokardinfarkt, der bei Frauen für 5,2 \% und bei Männern für 7,1 \% aller Todesfälle in Deutschland im Jahr 2013 verantwortlich war (Robert Koch-Institut 2015). Betrachtet man darüber hinaus die HerzKreislauf-Erkrankungen im Licht des immer stärker zunehmenden Kostendrucks im Gesundheitswesen, so zeigt sich, dass diese auch hier mit einem Anteil von 14,5\% an den direkten Krankheitskosten führend waren (Robert Koch-Institut 2015).

Aufgrund dieses hohen Stellenwertes der ischämischen Herzkrankheiten wird viel Aufmerksamkeit auf die Optimierung von Prävention, Diagnostik und Therapie gerichtet. Durch gezielte Maßnahmen in diesen Bereichen im Sinne einer leitlinienkonformen Diagnostik und Therapie, basierend auf Empfehlungen der European Society of Cardiology (ESC) (Steg et al. 2012), ist die Sterberate des Myokardinfarkts seit Jahren rückläufig. Sie fiel im Zeitraum von 1998 bis 2013 um ca. 50 \% (Robert Koch-Institut 2015). Ein großer Teil dieser Patientinnen und Patienten (zur besseren Lesbarkeit wird im Folgenden überwiegend die männliche Sprachform verwendet, soweit diese verwendet wird, gilt sie auch für die weibliche) verstirbt allerdings bereits, bevor überhaupt das Krankenhaus (KHS) erreicht wird. In dieser prähospitalen Phase liegt daher das größte Potenzial für mögliche Letalitätssenkungen (Hamm 2004b).

Nach Daten aus der gesetzlichen Qualitätssicherung lag die Rate von intra- oder postprozedural schweren kardialen und zerebrovaskulären Ereignissen (MACCE $=$ major adverse cardiac and cerebrovascular events) bei Patienten mit akutem ST-Strecken-Hebungsinfarkt $($ STEMI $=$ ST-segment elevation myocardial infarction), die im Rahmen einer perkutanen Koronarintervention $(\mathrm{PCI}=$ percutaneous coronary intervention) behandelt wurden, im Jahr 2015 in Deutschland bei 10,01\% (IQTIG - Institut für Qualitätssicherung und Transparenz im Gesundheitswesen 2016). Diese Rate an MACCE, bei der vor allem die Krankenhaussterblichkeit den Großteil der Ereignisse ausmacht, ist allerdings in den letzten Jahren kontinuierlich angestiegen (AQUA - Institut für angewandte 
Qualitätsförderung und Forschung im Gesundheitswesen GmbH 2014). Hierzu trägt unter anderem die vermehrt invasive Behandlung zunehmend älterer und kränkerer STEMIPatienten bei. Vor diesem Hintergrund kommt im Rahmen der STEMI-Versorgung der Bewertung des individuellen Patientenrisikos eine immer größere Bedeutung zu. Mit Hilfe von Risiko-Scores ist es dabei möglich, das individuelle Risiko zu definieren und auch in der Behandlung risikoadjustiert vorzugehen. Nach Angaben aus der Literatur haben STEMI-Patienten mit einer koronaren Mehrgefäßerkrankung eine erhöhte Sterblichkeit (Sorajja et al. 2007). Bisher ist allerdings nur wenig darüber bekannt, ob, und inwieweit die Prognose weiter verschlechtert wird, wenn bei diesen STEMI-Patienten zusätzlich ein bereits vorhandener chronischer Verschluss in einem Nicht-Infarkt-Gefäß vorliegt. Hierauf soll in der folgenden Arbeit der Fokus gelegt werden.

\subsection{Koronaranatomie und Versorgungstyp}

Als Vasa privata des Herzens haben die Koronargefäße die Aufgabe, den Herzmuskel mit ausreichend Blut und somit Sauerstoff zu versorgen, um die Pumpfunktion des Herzens aufrechtzuerhalten. Als funktionelle Endarterie kommt hierbei jedem einzelnen Gefäß eine besondere Rolle zu, da normalerweise kaum ausgeprägte Kollateralkreisläufe existieren und es im Falle eines Gefäßverschlusses schnell zu einer Unterversorgung des Myokards und somit zum Untergang von Herzmuskelzellen kommen kann (Drenckhahn 2004).

Das Koronarsystem gliedert sich in drei Hauptäste: den Ramus interventricularis anterior (RIVA) und den Ramus circumflexus (RCX) der linken Koronararterie (LCA = left coronary artery) sowie die rechte Koronararterie $(\mathrm{RCA}=$ right coronary artery). Die rechte und linke Koronararterie entspringen direkt der Aorta ascendens knapp oberhalb der Aortenklappe. Sie verlaufen epikardial und versorgen so das Myokard von außen nach innen (Baer und Rosenkranz 2011).

Zunächst verläuft die LCA meist als gemeinsamer Hauptstamm, der sich in der Regel schon nach wenigen Millimetern in den RIVA und den RCX aufteilt. Während der RIVA mit seinen Ästen vor allem die Vorder- und Lateralwand des linken Ventrikels (Ramus diagonalis) und das Septum (Rami septales) versorgt, unterhält der RCX über Marginal- und Posterolateraläste (PLA) größtenteils die Lateral- und Hinterwand des linken Ventrikels. Als relativ häufige Variante entspringt der LCA zwischen RIVA und RCX noch ein Ramus intermedius. Im Gegensatz zum relativ kurzen Verlauf der LCA verläuft die RCA zumeist langstreckig als eigenständiger Hauptstamm und gibt dabei nur wenige rechtsventrikuläre Äste ab. Im distalen Abschnitt teilt sich die RCA, sofern angelegt, in einen Ramus interventricularis posterior (RIVP) und einen rechten PLA, diese beiden Äste versorgen unter 
anderem den diaphragmalen Teil des Septums und des linken Ventrikels, im klinischen Gebrauch als Hinterwand bekannt. Des Weiteren gibt die RCA im proximalen Teil Äste zur Versorgung des rechten Atriums sowie die Sinusknotenarterie ab (Lapp und Krakau 2013).

Die Koronararterien weisen hinsichtlich ihrer Länge und des Gefäßdurchmessers interindividuell starke Unterschiede auf. Trotz dieser großen Anzahl an Variationsmöglichkeiten ist es möglich, die Koronarversorgung jeder Person einem bestimmten Versorgungstyp zuzuordnen. So wird in Literatur und Klinik ein Rechtsversorgungstyp von einem Linksversorgungstyp sowie von einem kodominanten Versorgungstyp unterschieden. Nach einer Arbeit von Daves (1970) wird der Verlauf der Koronararterien als ein Ring-SchleifenSystem bezeichnet, dessen Grundlagen ein Gefäßring an der Herzbasis und eine senkrecht dazu verlaufende Gefäßschleife sind. Der Ring an der Basis wird von der RCA im rechten und dem RCX im linken Sulcus atrioventricularis gebildet. Die senkrecht dazu verlaufende Schleife besteht aus dem RIVP der RCA und dem RIVA der LCA, die u. a. im Sulcus interventricularis in Richtung Herzspitze ziehen. Für die Definition des Versorgungstyps ist an dieser Stelle die Kreuzungsregion von Schleife und Ring, als Crux cordis bezeichnet, von großer Bedeutung. Hierbei unterliegen gerade die an der Herzbasis gelegenen Gefäße starken interindividuellen Unterschieden. Ihre Ausprägungen, vor allem im Hinblick auf die Gefäßlänge, variieren gegensinnig. Je nachdem, in welchem Ausmaß die RCA oder der RCX über die Crux cordis hinausziehen und somit für die Versorgung der linksventrikulären Hinterwand verantwortlich sind, kann man den einzelnen Versorgungstyp im Koronarangiogramm abgrenzen (Lapp und Krakau 2013).

Definitionsgemäß entspringen beim Linksversorgungstyp die PLA und der RIVP einem kaliberstarken RCX (Veltman et al. 2015). Die LCA reicht in diesem Fall über die Crux cordis hinaus und versorgt den kompletten linken und zusätzlich noch einige Abschnitte des rechten Ventrikels. Aufgrund dieser unausgeglichenen Myokardversorgung ist u. a. ein Linksversorgertyp beim akuten Koronarsyndrom mit einem signifikant schlechterem Outcome assoziiert (Goldberg et al. 2007; Kuno et al. 2013; Veltman et al. 2015).

Der Rechtsversorgungstyp hingegen ist durch eine stark ausgeprägte, über die Crux cordis hinausziehende RCA gekennzeichnet. Sie teilt sich in einen RIVP sowie in einige kräftige Posterolateraläste und versorgt somit Teile der Hinterwand und des Septums des linken Ventrikels, womit dessen Versorgung nicht nur von einem Gefäß abhängig ist. Patienten mit einem Rechtsversorgungstyp hätten somit bei einem Infarkt im Bereich der LCA noch eine gewisse Absicherung der Versorgung des linken Ventrikels über die RCA. Als kodominant bzw. ausgeglichen wird die Herzversorgung bezeichnet, wenn die kräftigen Posterolateraläste aus der linksseitigen RCX entspringen und der RIVP der RCA 
entstammt. Das Herz wird in diesem Fall von zwei ähnlich stark ausgeprägten Hauptstämmen versorgt, wobei die RCA den diaphragmalen Teil des interventrikulären Septums und der RCX die linksventrikuläre Hinterwand versorgt (Lapp und Krakau 2013).

Es zeigt sich aufgrund einer uneinheitlichen Definition der Versorgungstypen eine breite Schwankung in den Häufigkeitsangaben. In der aktuellen Literatur wird die Prävalenz des Rechtversorgungstyps mit ca. 86-90\% angegeben, während das Auftreten des Linksversorgertyps bei 8-10\% und der kodominanten Variante zwischen 2-5\% liegt (Angelini et al. 2002; Bazzocchi et al. 2011; Kuno et al. 2013; Veltman et al. 2015).

\subsection{Koronare Herzkrankheit}

\subsubsection{Definition und Pathogenese}

Bei der KHK handelt es sich um eine Stenosierung großer Herzkranzgefäße, die zu einer Myokardischämie und typischen Symptomen führen kann. Ursächlich hierfür ist ein Missverhältnis zwischen Sauerstoffangebot und -bedarf des Myokards, auch als Koronarinsuffizienz bezeichnet (Renz-Polster und Krautzig 2008). Das klinische Erscheinungsbild der KHK kann sich chronisch oder akut manifestieren. Es umfasst asymptomatische (stumme) Ischämien, eine in Art und Dauer des Auftretens stabile Angina Pectoris (AP), Herzrhythmusstörungen, die ischämische Herzinsuffizienz sowie das vital bedrohliche „akute Koronarsyndrom“ (ACS = acute coronary syndrome) und den plötzlichen Herztod (Herold 2014). Die Koronarinsuffizienz kann pathogenetisch auf verschiedene Ursachen zurückzuführen sein, in den allermeisten Fällen ist die Atherosklerose der großen Koronargefäße ursächlich für die KHK (Libby 2002).

Es konnten zahlreiche kardiovaskuläre Risikofaktoren identifiziert werden, die das Gefäßendothel schädigen und zur Entstehung der Atherosklerose beitragen. Hierzu zählen vor allem das Rauchen, die Arterielle Hypertonie, eine Hypercholesterinämie, Diabetes mellitus (DM), körperliche Inaktivität und (intraabdominelle) Adipositas sowie eine familiäre Disposition (Montalescot et al. 2013). Nachdem jahrelang die Ursache der Atherosklerose ausschließlich im krankhaft veränderten Auftreten der Blutfette vermutet wurde, zeigen neuere Studien, dass in den Entstehungsprozess neben Lipoproteinen vor allem Entzündungszellen des Blutes und Endothelzellen involviert sind, die auf komplexe Weise miteinander interagieren. Hierbei wird gerade der Entzündungsreaktion eine Schlüsselrolle in der Atherogenese zugeschrieben (Libby und Theroux 2005; Baer und Rosenkranz 2011). Begünstigt durch die Risikofaktoren kommt es anfänglich zu einer Beeinträchtigung der physiologischen Endothelfunktion und damit zu einer Anreicherung von LDLLipoproteinen in der Intima der Gefäßwand. Die dort gebundenen Lipoproteine werden in 
einen oxidierten Zustand versetzt und lösen so, unter anderem zytokinvermittelt, eine Entzündungsreaktion aus. Teil dieser Reaktion ist, neben einer weiteren Akkumulation von Lipoproteinen in der Gefäßintima, eine vermehrte Expression von LeukozytenAdhäsionsmolekülen (VCAM-1) sowie die Freisetzung chemotaktischer Substanzen, die dazu führen, dass Monozyten und T-Lymphozyten in die Intima eindringen können. Dabei kommt es zu einer ausgeprägten Kommunikation zwischen den eingewanderten Leukozyten und den Zellen der Gefäßintima, vornehmlich Endothel- und glatte Muskelzellen (smooth muscle cells). All diese Prozesse führen zu einer schleichenden Atherombildung (Libby und Theroux 2005; Baer und Rosenkranz 2011). In Gefäßintima und Media kommt es in der Folge zu einer direkten Verknüpfung beider pathogenetischer Ursachen, die den nächsten Schritt der Atherombildung charakterisiert: Die eingedrungenen Monozyten entwickeln sich zu Makrophagen und phagozytieren über Scavenger-Rezeptoren modifizierte Lipide und Lipoproteinbestandteile. Diese lipidangereicherten Makrophagen werden als Schaumzellen bezeichnet, imponieren im makroskopischen Bild als fatty streaks und lösen weitere entzündliche Reaktionen aus (Kröger und Libby 2011). So kommt es unter anderem, Mediator- und Wachstumsfaktor gekoppelt, zu einer Proliferation der fibrösen, extrazellulären Matrix, die mit einer Einwanderung von glatten Muskelzellen aus der Tunica media in die Gefäßintima einhergeht. Die hierbei entstehende fibrös-fettige Masse kennzeichnet die Entstehung einer stabilen atherosklerotischen Plaque und führt zu einer diskontinuierlichen Verdickung der Gefäßwand, welche das Gefäßlumen einengt und somit in einer signifikanten Stenose münden kann. Charakteristisch für diese stenosierenden, stabilen Läsionen ist vor allem eine dicke fibröse Kappe, die einen nur geringen Lipidpool umgibt (Libby 2002; Baer und Rosenkranz 2011). Durch die zunehmende Gefäßverengung kann es in Situationen, in denen der Sauerstoffbedarf des Myokards steigt, zu einer Minderversorgung des Herzmuskels kommen. Diese Myokardischämie ruft das klinische Bild einer häufig durch psychische oder physische Belastung ausgelösten stabilen AP hervor. Hierbei zeigt sich typischerweise ein retrosternaler, dumpfer oder drückender Brustschmerz, der in Hals, Unterkiefer, Schulter, linken Arm aber auch in das Epigastrium ausstrahlen kann und in der Regel durch Ruhe oder Nitroglyceringabe innerhalb weniger Minuten abklingt. Einige Patienten beklagen ausschließlich ein Druck- und Engegefühl bzw. ein Brennen im Thorax. Neben diesem Leitsymptom treten häufig noch weitere, eher unspezifische Symptome auf. So kann es zu Übelkeit und Schwindel sowie Atemnot kommen (Montalescot et al. 2013).

Akute und lebensgefährliche Ereignisse dagegen haben ihre Ursache überwiegend in einer Plaqueruptur (Falk et al. 1995). Meist geht diese von nicht-stenosierenden atherosklerotischen Läsionen aus, die aus einer dünn fibrös umhüllten Kappe mit einem lipid- 
reichen Zentrum bestehen („,komplizierte Läsion“). Am Ort dieser instabilen Läsion ist die Gefäßwand nur wenig elastisch, sodass es schnell zu Einrissen in der dünnen Plaquedecke kommen kann. Sobald diese Plaque einreißt, häufig ausgelöst durch Stresssituationen, werden an dieser Stelle Thrombozyten aktiviert und die Gerinnungskaskade mit Thrombusbildung in Gang gesetzt (Davies 2000; Libby 2002; Baer und Rosenkranz 2011). Der gebildete Thrombus kann einen kompletten Gefäßverschluss und damit eine absolute Myokardischämie zur Folge haben, welche schon nach ca. 10 Minuten zu elektronenmikroskopisch sichtbaren Veränderungen an den Zellorganellen führt. Bereits nach 30 Minuten sind die ersten Myokardschäden irreversibel und es kommt zum beginnenden nekrotischen Untergang von Myozyten im Sinne eines Herzinfarkts. Dieser Zelluntergang manifestiert sich im Stadium der Koagulationsnekrose nach etwa 4-6 h. Im Verlauf über mehrere Wochen kommt es dann zu einem nicht regenerationsfähigen Umbau des Herzmuskelgewebes zu Narbengewebe mit daraus resultierender Abnahme der Kontraktilität, die in einer Herzinsuffizienz münden kann (Bültmann et al. 2008). Je schneller das verschlossene Gefäß wiedereröffnet wird, desto geringer sind die Schäden am Myokard.

\subsubsection{Koronarstenosen}

Im Falle einer Stenosierung der Herzkranzgefäße kommt es aufgrund des reduzierten Blutdrucks in den distal der Stenose gelegenen Abschnitten zu einer verminderten Koronardurchblutung. Physiologischerweise kann die Koronardurchblutung z. B. in Belastungssituationen um das Vierfache gesteigert werden, um eine ausreichende Perfusion des Myokards zu gewährleisten (Koronarreserve). Auch bei stenosierten Gefäßen wird durch eine kompensatorische Dilatation und eine damit einhergehende Abnahme des Widerstands der poststenotischen Gefäßabschnitte dafür gesorgt, dass die Durchblutung der Koronarien, trotz reduzierten Perfusionsdrucks, aufrechterhalten bleibt. Bemerkbar macht sich eine Stenosierung erst ab einer Lumenverengung des Gefäßes von über $50 \%$. In diesem Falle reicht die kompensatorische Dilatation nicht mehr aus, um die maximale Koronardurchblutung zu gewährleisten. Somit kann der noch vorhandene Blutfluss in körperlichen Stresssituationen nicht mehr den Sauerstoffbedarf des Myokards decken. Ab einer Lumeneinengung von $>75 \%$ kommt es zu einer belastungsabhängigen Ischämie mit der typischen AP-Symptomatik (Lapp und Krakau 2013).

Zur Klassifikation der Koronarstenosen werden koronarangiographisch unterschiedliche Merkmale betrachtet. Zur Beurteilung einer Engstelle wird vom Untersucher eine Stenosegradeinteilung vorgenommen. Während eine Stenosierung $<25 \%$ als Wandunregelmäßigkeit beschrieben wird, spricht man ab einer Stenosierung $>90 \%$ von einer höchstgradigen Stenose (Hamm et al. 2008). Der Stenosegrad ist allerdings nicht mit der eigentlichen 
Lumeneinengung gleichzusetzen, sondern betrifft lediglich die Reduktion des Durchmessers. Daneben kann der noch vorhandene Koronarfluss über der Stenose- bzw. Verschlussstelle nach der TIMI (Thrombolysis in Myocardial Infarction)-Klassifikation (TIMI Grade Flow) betrachtet werden (Chesebro et al. 1987). Diese Beurteilung findet vor allem bei Patienten mit akutem Myokardinfarkt Anwendung und kann zudem zur Erfolgskontrolle nach Koronarintervention dienen. Ist kein Koronarfluss über die Stenosebzw. Verschlussstelle hinaus zu ermitteln liegt ein TIMI-0-Fluss vor. Ein TIMI-III-Fluss dagegen bezeichnet eine vollständige und umgehende Perfusion des gesamten Gefäßes.

Als weiteres Klassifikationsmerkmal gilt die Stenosemorphologie nach den Kriterien der American Heart Association (AHA) und des American College of Cardiology (ACC). Hierbei wird die Engstelle unter anderem nach Länge, Kontur, Lage im Gefäß und Grad der Verkalkung beurteilt. Daneben ist auch die Lokalisation der Stenose von großer Bedeutung für Therapie und Prognose, je weiter proximal diese liegt, umso größer ist das abhängige Versorgungsgebiet (Ryan et al. 1993). Hinsichtlich der Anzahl der von einer Stenosierung $>50 \%$ betroffenen großen epikardialen Gefäße (RCX, RIVA und RCA) kann man die koronare Herzkrankheit in eine 1-Gefäß-, 2-Gefäß- oder 3-Gefäß-KHK einteilen und so den Gefäßerkrankungstyp festlegen. Das Ausmaß der Gefäßerkrankung ist entscheidend für die Prognose. Patienten mit einer 3-Gefäß-KHK haben dabei allgemein eine ungünstigere Prognose (Emond et al. 1994).

Die Zusammenschau all dieser Klassifikationsparameter trägt dann, unter Berücksichtigung weiterer individueller Risikofaktoren des Patienten, maßgeblich zur Prognosebeurteilung und zur Therapieentscheidung (konservativ vs. katheter-interventionell vs. operativ) bei.

\subsubsection{Chronische Koronargefäßverschlüsse}

Eine Maximalausprägung der koronaren Herzerkrankung ist der vollständige Verschluss eines Koronargefäßes. Ein Verschluss kann zum einen akut als Auslöser des ACS, zum anderen bei Fortbestehen über einen längeren Zeitraum als chronischer Totalverschluss (chronic total occlusion $=$ CTO) in Erscheinung treten.

In einem Konsensdokument des EuroCTO Clubs wird eine Läsion als CTO bezeichnet, wenn in dem betroffenen verschlossenen Segment ein TIMI-0-Fluss vorherrscht und der Verschluss seit mindestens drei Monaten besteht (Di Mario et al. 2007; Sianos et al. 2012). Allerdings ist die genaue Bestimmung der Verschlussdauer schwierig und stützt sich, sofern kein angiographischer Beweis auf Grundlage älterer Befunde vorliegt, meist auf Schätzungen, die sich aus angiographischen (z. B. ausgeprägte Verkalkung des Verschlusses und Kollateralisierung) oder klinischen Informationen ergeben. So können vor allem ein anamnestisch gesicherter alter Myokardinfarkt oder auch pathologische Q-Zacken im 
Elektrokardiogramm (EKG) auf einen chronischen Verschluss hinweisen. Allerdings weist nur ein geringer Anteil der CTO-Patienten diese Merkmale auf, bei lediglich $26 \%$ der CTO-Patienten konnten Q-Zacken über dem CTO-Areal registriert werden und nur $40 \%$ der CTO-Patienten gaben anamnestisch einen Myokardinfarkt an (Fefer et al. 2012).

Aufgrund der häufig asymptomatischen Manifestation eines CTO ist die genaue Prävalenz schwer zu beziffern. Laut einer kanadischen Studie hatten $18 \%$ aller Patienten mit einer KHK einen CTO (Fefer et al. 2012). Christofferson et al. (2005) fanden bei $52 \%$ ihrer untersuchten Patienten mit einer $>70 \%$ des Gefäßlumens einengenden Stenose einen CTO. Bei diesen Patienten war von einem chronischen Verschluss bei $64 \%$ die RCA, bei $35 \%$ der Patienten der RCX und bei $28 \%$ der Patienten der RIVA betroffen, dabei konnte bei $12 \%$ aller untersuchten Patienten mehr als ein CTO nachgewiesen werden.

Ein Großteil (70\%) der symptomatischen CTO-Patienten, die mit einer PCI behandelt wurden, fiel mit einer stabilen AP oder einer Herzinsuffizienz mit ischämischer Genese auf, $18 \%$ der Patienten klagten über die Symptome einer instabilen AP (Olivari et al. 2003) und bei einem kleineren Teil wird ein CTO als zusätzlicher Befund im Rahmen eines ACS mit culprit lesion in einem anderen Gefäßareal diagnostiziert.

Eine katheter-interventionelle Therapie des CTO gilt als sehr anspruchsvoll, da aufgrund der histologischen Beschaffenheit des Verschlusses eine Drahtpassage relativ häufig nicht gelingt. Die komplexe Histopathologie des CTO spielt daher eine zentrale Rolle in der Entwicklung neuer Rekanalisierungstechniken (Irving 2014). Erste post-mortem Studien mit Blick auf CTOs wurden in den 1990er Jahren veröffentlicht und zeigten einen Einblick in die Histologie chronischer Verschlüsse (Katsuragawa et al. 1993). Üblicherweise besteht eine CTO-Läsion aus einem Mix aus Kalk, extrazellulärer Matrix, Fettzellen und glatten Muskelzellen, wobei sich die dichtesten, stark verkalkten Abschnitte jeweils am proximalen und distalen Ende (Kappen) der Plaque befinden und einen eher weichen Kern aus Fettzellen und Thromben umgeben (Stone et al. 2005). Die Verkalkung ist dabei im Bereich des proximalen Verschlussendes (proximal cap) besonders ausgeprägt und bei angiographisch stumpf endenden Läsionen am stärksten (Fujii et al. 2006). Neben dieser starken Verkalkung zählt eine Entzündungsreaktion in der Gefäßintima sowie eine ausgeprägte Neovaskularisierung ausgehend von den Vasa vasorum der Gefäßadventitia zu den typischen Merkmalen eines CTO. Diese neugebildeten Gefäßkanäle können dabei eine Revaskularisierung des distalen Gefäßlumens ermöglichen und auch therapeutisch als Leitstruktur genutzt werden. Srivatsa et al. (1997) gelang es zudem zu zeigen, dass CTOLäsionen biologisch aktiv sind und somit altersabhängig charakterisiert werden können. Je älter die Läsion ist, desto größer ist der bindegewebige, verkalkte Anteil, während jüngere Läsionen eher weich sind und daher eine höhere Rekanalisierungswahrscheinlichkeit 
besitzen. Unter Anwendung einer virtuellen Histologie im Rahmen des intravaskulären Ultraschalls konnten Guo et al. (2013) in vivo wichtige Informationen zur Genese chronischer Verschlüsse gewinnen. Nach Analyse der Plaquebestandteile konnte gezeigt werden, dass $84 \%$ der untersuchten CTOs hinsichtlich der Plaquezusammensetzung mit akuten Koronarverschlüssen vergleichbar sind. Die Autoren gehen somit davon aus, dass der größte Teil aller CTOs aus akuten Ereignissen im Sinne einer Plaqueruptur mit anschließender Thrombusbildung hervorgeht. Der hieraus resultierende Myokardinfarkt wurde dabei entweder nicht adäquat behandelt oder es konnte sich aufgrund einer prompten Kollateralversorgung trotz Verschluss kein akuter Infarkt ausbilden. Die restlichen $16 \%$ dieser untersuchten CTOs scheinen dagegen ihren Ursprung in einer langsam voranschreitenden Atherosklerose mit zunehmender Stenosierung des Koronargefäßes zu haben (Irving 2014).

Ein CTO wird fälschlicherweise häufig als eine gutartige Läsion gesehen, die u. a. aufgrund ausreichender Kollateralisierung scheinbar selten zu Beschwerden führt und somit keiner interventionellen Therapie bedarf. Dieser Eindruck wird allerdings von verschiedensten Studien widerlegt (Carlino et al. 2015). Wie bereits erwähnt, besteht bei einigen Patienten eine gewisse klinische Symptomatik. Einige Autoren konnten zudem zeigen, dass das vom CTO-Gefäß versorgte Myokardareal einer Ischämiezone mit möglichem Zelluntergang entspricht (Sachdeva et al. 2014) und darüber hinaus, dass eine KHK mit CTO insgesamt mit einer schlechteren Prognose einhergeht als eine KHK ohne CTO (Carlino et al. 2015). Bleiben daher CTO-Patienten auch unter maximal medikamentöser Therapie symptomatisch und zeigt sich zudem ein großes Ischämieareal, käme nach individueller Abwägung klinischer und angiographischer Befunde eine Revaskularisierung in Betracht (Sianos et al. 2012). Zu den invasiven Therapieoptionen zählt hierbei neben der PCI vor allem die Bypass-OP. Nach Fefer et al. (2012) wurden $64 \%$ aller KHK-Patienten mit CTO medikamentös behandelt, $26 \%$ unterzogen sich einer Bypass-OP und lediglich $10 \%$ aller CTO-Patienten wurden per PCI therapiert. Diese geringe PCI-Rate lässt sich damit erklären, dass die komplexe Behandlung eines CTO aufgrund der genannten histopathologischen Merkmale mit geringeren Erfolgs- ( $72 \%$ bei CTO-PCI vs. $\sim 97 \%$ bei Nicht-CTO-PCI (Abbott et al. 2006)) und höheren Komplikationsraten einhergeht. Oftmals ist es nicht möglich, die Läsion mit einem Draht zu passieren, in anderen Fällen wird der Draht in die Gefäßwand umgelenkt und verursacht Gefäßdissektionen bis hin zur Perforation (Lapp und Krakau 2013). Zudem ist die CTO-PCI im Vergleich zu Routinekoronarinterventionen sehr zeit- und ressourcenaufwändig. Dies führt zwangsläufig zu einer erhöhten Strahlenbelastung für Patient (4,6 Gy bei CTO-PCI vs. 1,2 Gy bei 1Gefäß-PCI (Suzuki et al. 2006)) und Personal und erfordert eine höhere Menge an 
Kontrastmittel, was das Risiko einer kontrastmittelinduzierten Nephropathie erhöht (Shah 2011; Carlino et al. 2015).

Dennoch können CTO-Patienten in vielerlei Hinsicht von einer PCI-Therapie profitieren. So führt eine erfolgreiche CTO-PCI im Vergleich zu einem erfolglosem Versuch zu einer Reduktion der AP-Symptomatik (Joyal et al. 2010) und zu einer verbesserten LV (linksventrikulären)-Funktion (Baks et al. 2006; Kirschbaum et al. 2008). Vor allem aber zeigt sich nach gelungener CTO-PCI eine Reduktion der Langzeitmortalität (Suero et al. 2001; Khan et al. 2013) und die Inzidenz einer Bypass-OP nimmt nach erfolgreicher CTO-PCI ab (Yamamoto et al. 2013). Bei niedrigen Patientenzahlen und fehlender Randomisierung ist allerdings die Aussagekraft all dieser Studien begrenzt und zeigt lediglich eine Tendenz an. Zudem wurden bisher nur erfolgreiche mit erfolglosen CTO-PCI-Therapien verglichen, der randomisierte Vergleich mit einer medikamentösen bzw. Bypass-Therapie steht weiterhin aus (Carlino et al. 2015).

Mit wachsender Erfahrung und Einführung neuer PCI-Techniken und Technologien sowie Fortschritten in Bildgebung und Diagnostik, die zu einer verbesserten Interventionsplanung beitragen, steigt die Rate erfolgreicher und komplikationsloser Rekanalisierungen per PCI zusehends. In einigen auf CTOs spezialisierten PCI-Zentren werden mittlerweile Erfolgsraten von 85-90 \% erreicht (Thompson et al. 2009; Whitlow et al. 2012), zudem sinken die Komplikationsraten auf das Niveau von Routineeingriffen (Rathore et al. 2009; Carlino et al. 2015). Diese Zahlen zeigen, dass eine CTO-PCI vor allem in spezialisierten Zentren mit erfahrenen Untersuchern auch im klinischen Alltag eine bedeutende Rolle spielen kann. Bisher gibt es allerdings aufgrund der eher schwachen Studienlage keine eindeutige Leitlinienempfehlung zur Revaskularisierung von chronischen Verschlüssen. Laut den aktuellen europäischen Leitlinien sollte eine CTO-Revaskularisierung per PCI im Falle einer entsprechenden Symptomatik sowie beim Nachweis einer Ischämie bzw. Myokardvitalität im CTO-Areal in Betracht gezogen werden. Bei komplexen Mehrgefäßerkrankungen mit hohem SYNTAX Score, häufig resultierend aus einem CTO, wird dennoch weiterhin die Bypass-OP als Therapie der Wahl empfohlen (Windecker et al. 2014). Allerdings ist auch in solchen Fällen eine vollständige Revaskularisierung per PCI durchaus möglich (Carlino et al. 2015).

Die PCI ist somit bei der individuellen interdisziplinären CTO-Therapieentscheidung, zumindest in erfahrenen und spezialisierten Zentren, neben der Bypass-OP auch immer eine Option. Hierbei wird im Falle einer Mehrgefäß-KHK mit CTO zu einem etappenweisen Vorgehen geraten, um den Umfang des einzelnen Eingriffs zu begrenzen (Sianos et al. 2012). Von größter Bedeutung in der CTO-Therapie ist jedoch die Identifikation solcher Patienten, die maximal von einem Eingriff profitieren würden. Hierzu zählen vor allem 
symptomatische Patienten mit Ischämienachweis im CTO-Areal und einer erhaltenen Myokardvitalität. Zudem ist zu beachten, dass eine exakte präprozedurale CTO-Diagnostik zur Generierung eines individuellen Risikoprofils eine entscheidende Rolle in der Versorgung eines CTO-Patienten spielt. Im Einklang mit der Erfahrung von CTOExperten kann so im Rahmen einer PCI die Erfolgsrate steigen und die Komplikationsrate sowie die Interventionszeit abnehmen. Letzteres führt zudem - bei strikter Einhaltung des Strahlenschutzes - zu einer Verringerung der Strahlenbelastung für Personal und Patient.

\subsubsection{Kollateralversorgung}

Wie bereits erwähnt, gehen chronische Koronargefäßverschlüsse oftmals mit einer ausgeprägten interarteriellen Kollateralisierung einher. Auch bei gesunden Menschen ist dieses Kollateralnetzwerk bereits angelegt, allerdings ist es nur gering entwickelt und nicht in der Lage, eine adäquate Blutversorgung zwischen den großen epikardialen Koronargefäßen zu gewährleisten. Kommt es aber zum Verschluss einer Koronararterie, ändern sich die Druckverhältnisse im gesamten Koronarversorgungsgebiet. Dies führt, ausgelöst durch endotheliale Scherkräfte im Kollateralgefäß und eine Sekretion von Wachstumsfaktoren, zu einer Dilatation und einem remodelling von kleinen Kollateralarteriolen zu kaliberstarken Arterien (Schaper 2009). Der Zeitraum, in dem sich diese Entwicklung abspielt, ist individuell höchst variabel. Untersuchungen zeigen, dass sich im Regelfall die Kollateralen in einer Phase von zwei bis zwölf Wochen nach einem Verschluss herausbilden (Werner et al. 2003). Bei manchen Patienten ist das Netzwerk aber schon im gesunden Zustand so stark angelegt, dass sich im Falle eines kompletten Verschlusses sofort kaliberstarke Kollateralgefäße entfalten (Wustmann et al. 2003). So konnte gezeigt werden, dass CTOPatienten ohne detektierbare Infarktgeschichte (leere Anamnese und keine Q-Zacken) ein besonders ausgeprägtes und vollständig entwickeltes kollaterales Netzwerk aufwiesen. Dieses konnte bei Manifestation des kompletten Verschlusses die Blutversorgung der distalen Segmente des betroffenen Koronargefäßes übernehmen und so eine Infarzierung verhindern. Bei CTO-Patienten mit Hinweisen auf einen alten Infarkt hingegen war das angelegte Kollateralnetz vermutlich zu schwach, um einen Infarkt zu verhindern. Hier konnte sich ein adäquates Kollateralnetzwerk erst im Verlauf über mehrere Wochen nach dem Verschlussereignis entwickeln. Je älter der Verschluss hierbei ist, umso ausgereifter ist das Kollateralnetz (Werner et al. 2003). Eine Metaanalyse konnte zeigen, dass KHKPatienten mit einer guten Kollateralversorgung im Vergleich zu Patienten mit einem schwachen Kollateralnetz einen signifikanten Überlebensvorteil aufweisen (Meier et al. 2012; Irving 2014). 
Der Nachweis von Kollateralen in der koronarangiographischen Diagnostik ist allerdings limitiert, da sie aufgrund einer Größe von 40-200 $\mu \mathrm{m}$ hauptsächlich unterhalb des Auflösungsvermögens moderner Bildgebungssysteme liegen (Werner 2014). Zur einheitlichen Bewertung des Kollateralstatus im angiographischen Bild spielen vor allem zwei ScoringSysteme eine wichtige Rolle. Hierzu zählt zum einen der Rentrop Score, welcher allerdings nicht die Kollaterale an sich sondern die retrograde Füllung des distal eines Verschlusses gelegenen Gefäßsegments mit Kontrastmittel beurteilt: Grad $0=$ keine sichtbare Kollateralfüllung; Grad 1 = kollaterale Füllung von Seitästen, aber nicht des verschlossenen Gefäßes; Grad 2 = teilweise kollaterale Füllung des verschlossenen Gefäßsegments; Grad 3 $=$ komplette kollaterale Füllung des verschlossenen Gefäßsegments (Rentrop et al. 1985). Laut Werner (2014) konnte bei 85 \% aller Patienten mit CTO eine Kollateralversorgung mit einem Rentrop Score von 3 beobachtet werden.

Um das Ausmaß und die Morphologie der eigentlichen Kollaterale, auch im Hinblick auf eine mögliche retrograde PCI bei CTO, einheitlich und differenzierter beurteilen zu können, wurde eine Kollateralverbindungs-Klassifizierung $(\mathrm{CC}$ grade $=$ collateral connection grade) entwickelt (Werner et al. 2003): CC0 = keine kontinuierliche Verbindung zwischen Spender- und Empfängerarterie (14 \% der Patienten); CC1 = kontinuierliche, fadenförmige Verbindung (51\%); CC2 = kontinuierliche, seitastförmige Verbindung (35\%). Abhängig von der Art der führenden Kollateralverbindung unterschieden die Autoren zudem zwischen intrakoronaren Brückenkollateralen (6\% der Patienten), septalen Verbindungen zwischen zwei unterschiedlichen Koronargefäßen (44\%) sowie epikardialen (interarteriellen) Kollateralen mit proximalem (32\%) bzw. distalem Abgang (18\%).

Trotz der Fähigkeit, Infarkte zu verhindern und die Pumpfunktion zu erhalten, sind selbst stark ausgeprägte Kollateralen nicht in der Lage, einen suffizienten Blutfluss auch unter Belastung aufrechtzuerhalten. So kommt es bei mehr als $90 \%$ der Patienten mit normaler linksventrikulärer Pumpfunktion und gut kollateralisiertem СTO zu einer typischen Belastungsischämie, die eine therapeutische Intervention rechtfertigen würde (Werner et al. 2006; Werner 2014). Zu beachten ist hierbei, dass sich nach einer erfolgreichen CTO-PCI, mit Wiederherstellung des antegraden Flusses, die distal des CTO gelegene Kollateralverbindung wieder zurückbildet (Regress). Im Rahmen eines Re-Verschlusses des ehemaligen CTO-Gefäßes könnte dies einen Infarkt zur Folge haben, da die Wiedereröffnung der Kollaterale nur verzögert abläuft. Eine prä-interventionelle Kollateraldiagnostik ist in diesem Falle essentiell, so werden z. B. vormals als CC2 gewertete Kollateralen bei einem erneuten Verschluss schneller rekrutiert als CCO-Kollateralen (Werner et al. 2003; Irving 2014). Eine möglichst exakte Erfassung des Kollateralstatus bei Patienten mit KHK ist somit von größter Bedeutung, da sie zur Vervollständigung des 
individuellen Risikoprofils beiträgt und wichtige Informationen über mögliche Interventionsvarianten und die Prognose liefert.

\subsection{Akutes Koronarsyndrom und ST-Strecken-Hebungsinfarkt}

\subsubsection{Definition}

Unter einem akuten Koronarsyndrom versteht man die unmittelbar lebensbedrohliche Manifestation der KHK. Der Begriff ist in der Klinik vor allem als eine Arbeitsdiagnose zu verstehen, die ein Spektrum mit unterschiedlicher Ausprägung der akuten Myokardischämie beschreibt. Per Definition fallen unter diesen Ausdruck die Instabile AP ohne Troponin-Anstieg, der Nicht-ST-Strecken-Hebungsinfarkt (NSTEMI) bei Instabiler AP mit Troponin-Erhöhung und der STEMI (Hamm 2004a). Diesen drei Erscheinungsbildern liegt häufig die erwähnte Pathophysiologie der Plaqueruptur zugrunde. Ein hierdurch hervorgerufener vollständiger Gefäßverschluss hat meistens einen STEMI zur Folge, während inkomplette Verschlüsse eher einen NSTEMI oder eine instabile AP verursachen.

Die Begriffsdefinition des Myokardinfarktes wurde in den letzten Jahre laufend aktualisiert. Ursächlich hierfür war vor allem die ständige Weiterentwicklung diagnostischer und therapeutischer Verfahren, die es ermöglichte, immer sensitiver kleinere Infarktbereiche zu detektieren. Anfangs wurde von Seiten der WHO (World Health Organization) seit den 1970er Jahren der Ausdruck Myokardinfarkt bis hin zum Jahr 2000 als ein Syndrom, basierend auf: (1) der klinischen Symptomatik, (2) einem typischen Enzymanstieg und (3) dem typischen Infarkt-EKG, bezeichnet. Waren zwei von diesen drei Merkmalen positiv, wurde von einem Myokardinfarkt gesprochen (Bernard et al. 1979; Mendis et al. 2011). Um dem diagnostischen Fortschritt und der Handhabung des Begriffs im klinischen Alltag, aber auch im Hinblick auf wissenschaftliche Projekte, gerecht zu werden, wurde dieser in den letzten Jahren immer präziser definiert. Im Jahr 2000 wurde im Anschluss an eine ESC/ACC Konsens-Konferenz eine Neu-Definition des Myokardinfarkts vorgelegt (Alpert und Thygesen 2000). Hierbei wurde vor allem aus pathologischer Sicht jeder irreversible Zelluntergang auf dem Boden einer Myokardischämie als Myokardinfarkt definiert. Neben dieser pathologischen Definition wurden Kriterien benannt, die es ermöglichen, diesen Zelluntergang zu detektieren und es somit erlauben, die Diagnose Myokardinfarkt zu stellen (beruhend auf kardialer Biomarkerdiagnostik, EKG und Bildgebung). Für die Klinik hatte dies vor allem eine Neuklassifikation des Begriffs „Akutes Koronarsyndrom (ACS)“ mit den drei oben genannten Ausprägungen zur Folge.

Zur bestmöglichen Abbildung des wissenschaftlichen Fortschritts in der Medizin wurde nach mehreren Überarbeitungen und unter Einbeziehung verschiedener Gesellschaften 
und Gruppierungen im Jahr 2007 die universal definition of myocardial infarction veröffentlicht (Thygesen et al. 2007). Dieser Expertenkonsens erfährt seither eine regelmäßige Aktualisierung, im Jahr 2012 wurde die aktuell gültige Version veröffentlicht (Thygesen et al. 2012). Hauptkriterium für die Diagnose Myokardinfarkt ist hierbei der Nachweis von kardialem Troponin als sensitiver und spezifischer Marker einer Herzmuskelzellnekrose. Dabei ist es wichtig, den eigentlichen Myokardinfarkt von einem Myokardschaden, der nicht durch eine akute Myokardischämie hervorgerufen wurde, zu unterscheiden. Um dies zu gewährleisten, müssen klinische Ischämienachweise berücksichtigt werden, zudem wurden laborchemisch Grenzwerte entsprechend angepasst.

Nach der neuen Definition spricht man von einem akuten Myokardinfarkt, wenn im Rahmen des klinischen Bildes einer Ischämie eine Myokardzellnekrose nachgewiesen werden kann. Dies ist u. a. bei den folgenden Kriterien der Fall (Thygesen et al. 2012):

(1) Kardialer Biomarkeranstieg oder -Abfall (vor allem Troponin), hierbei muss mindestens ein Wert über der 99. Perzentile des oberen Referenzbereiches (ORB) liegen und zudem eines der folgenden Kriterien zutreffen:

- Ischämiesymptomatik

- neu aufgetretene ST-Hebungen oder neu aufgetretener Linksschenkelblock

- Q-Zacken-Entwicklung im EKG

- Nachweis eines Verlustes von Myokardvitalität oder neue Wandbewegungsstörungen in der Bildgebung

- angiographischer oder autoptischer Nachweis eines intrakoronaren Thrombus

(2) Plötzlicher Herztod mit Symptomen, die auf eine Myokardischämie hinweisen sowie vermutlich neu aufgetretene ischämische EKG-Veränderungen. Hierbei trat der Tod vor Bestimmung der kardialen Biomarker ein.

\subsubsection{Symptomatik}

Bei allen drei Formen des eingangs erwähnten ACS gilt als Leitsymptom die eher unspezifische, ischämische Symptomatik. Eine Differenzierung allein aufgrund der klinischen Präsentation des Patienten ist somit nicht möglich. Zu der Symptomatik zählt vor allem der Brustschmerz mit brennendem Charakter sowie das retrosternale Enge- bzw. Druckgefühl. Im akuten Fall sind diese Symptome intensiver, länger anhaltend (meist $>20$ min) und durch Einhalten von Ruhe oder Gabe von Nitroglycerin wenig zu beeinflussen. Häufig kommt es zu einer Ausstrahlung der Beschwerden in Unterkiefer, Hals, Nacken, Schulterbereich, in den linken bzw. seltener rechten Arm sowie in den Oberbauch. Darüber hinaus berichten Betroffene oftmals von einer Begleitsymptomatik in Form von Dyspnoe, Unruhe, Schwitzen, Übelkeit und Todesangst. Dieser typische Verlauf variiert 
allerdings interindividuell sehr stark. Während es bei Frauen häufig zu sehr unspezifischen vegetativen Symptomen und Bauch-, sowie Zahn- und Kieferschmerzen kommt, kann in einigen Fällen, z. B. bei Diabetikern, der Thoraxschmerz sogar komplett fehlen (Hamm 2004b). In der akuten Phase kommt es zudem sehr häufig zu lebensbedrohlichen Komplikationen wie Herzrhythmusstörungen, Symptomen einer Linksherzinsuffizienz (z. B. Luftnot) und einem Blutdruckabfall, der in einem kardiogenen Schock münden kann. Zu den subakuten bzw. Spätkomplikationen ( $>48$ h nach Symptombeginn) zählen vor allem die Herzwand- und Ventrikelseptumruptur, die Mitralklappeninsuffizienz, das Herzwandaneurysma, arterielle Embolien, eine Perikarditis sowie Arrhythmien und die Herzinsuffizienz (Steg et al. 2012).

\subsubsection{Diagnostik}

Im Falle des ACS ist eine schnelle und zielgerichtete Diagnostik von größter Bedeutung. Ziel ist es dabei, unter den Patienten mit ischämischer Symptomatik jene herauszufiltern, die an einem akuten Myokardinfarkt leiden und somit schnellstmöglich einer Reperfusionstherapie zugeführt werden müssen. Der erste Schritt zur Diagnose ist bei der oben genannten klinischen Präsentation neben Anamnese und klinischer Untersuchung das 12Kanal-Ruhe-EKG. Dem EKG kommt hierbei die zentrale Bedeutung zu. Es sollte augenblicklich, d. h. innerhalb von 10 min nach medizinischem Erstkontakt geschrieben und ausgewertet werden, da hier sofort zwischen einem STEMI und den anderen Varianten des ACS differenziert werden kann. Beim STEMI tritt charakteristischerweise eine am J-Punkt gemessene ST-Strecken-Hebung in zwei zusammenhängenden Ableitungen oder ein neu aufgetretener Linksschenkelblock auf (Steg et al. 2012).

Der Infarkt lässt sich anhand des EKG zeitlich in verschiedene Phasen einteilen. Bei nachgewiesenen ST-Hebungen spricht man vom Akutstadium (Stadium 1), das innerhalb von wenigen Minuten nach Gefäßverschluss auftritt. Dem geht das Ischämiestadium (Stadium 0) mit einer hochpositiven T-Welle (Erstickungs-T) voraus. Das Stadium 2 kennzeichnet eine beginnende Nekrose und ist charakterisiert durch eine Rückbildung der ST-Hebung und einer sich ausbildenden pathologischen Q-Zacke sowie einer negativen TWelle. Zu diesen Veränderungen kommt es in der Regel innerhalb von Stunden bis Tagen nach einem abgelaufenen Infarkt im Falle eines persistierenden Gefäßverschlusses. Auch Wochen bis Monate nach dem akuten Ereignis ist typischerweise noch ein pathologisches Q zu identifizieren, während sich die T-Negativierung zumeist wieder normalisiert (Stadium 3 und 4). Das Vorhandensein einer Q-Zacke kann somit als Hinweis auf einen alten, abgelaufenen Infarkt dienen (Hamm und Willems 2014). Neben dieser zeitlichen Komponente ist es aber auch möglich, anhand des EKG eine Aussage zur 
Infarktlokalisation und zur betroffenen Koronararterie zu treffen. Da sich der Myokardinfarkt meistens isoliert aufgrund eines verschlossenen Koronargefäßes in einem ganz bestimmten Versorgungsgebiet abspielt, lässt sich die EKG-Veränderung vor allem in den damit assoziierten Ableitungen nachweisen (Dill et al. 2013).

Während man den NSTEMI von der instabilen AP durch den Anstieg biochemischer Marker abgrenzt, reicht zur Diagnostik des STEMI und somit zur Indikationsstellung einer Revaskularisierungstherapie das EKG und die klinische Symptomatik aus. Da im Falle eines STEMI die schnellstmögliche Reperfusion des Gefäßes angestrebt wird und ein Biomarkeranstieg erst verspätet nachzuweisen ist, wird leitliniengerecht die Labordiagnostik nicht abgewartet, um die rasche Therapie nicht zu verzögern (Steg et al. 2012).

\subsubsection{Leitlinienkonforme STEMI-Therapie}

Ziel der Therapie beim akuten STEMI ist es, eine schnellstmögliche Rekanalisierung des betroffenen Infarktgefäßes zu realisieren, um die Dimension der Myokardschädigung so gering wie möglich zu halten. Schon Ende der 1970er Jahre konnten Reimer und Jennings (1979) belegen, dass das Ausmaß der Nekrose im Infarktgebiet maßgeblich von der Verschlussdauer bzw. vom Zeitpunkt der Reperfusion abhängt. Je schneller der Blutfluss im betroffenen Infarktgefäß wiederhergestellt ist, umso geringer ist der nekrotische Anteil im gefährdeten Areal. Der Zeitfaktor spielt daher in der Therapie eine übergeordnete Rolle, der größte Therapieeffekt wird für die ersten 2-3 h nach Symptombeginn angegeben. Nach Ablauf dieser kritischen Periode scheint ein Therapieerfolg nur noch einen geringen Einfluss auf die Mortalität zu haben (Gersh et al. 2005).

Die Leitlinien zur Versorgung von STEMI-Patienten werden regelmäßig aktualisiert und in Form von Pocket-Leitlinien an die Situation in Deutschland angepasst. Die aktuellen Leitlinien der ESC stammen aus dem Jahr 2012 (Steg et al. 2012). Mit Blick auf die Therapie des akuten Koronarsyndroms gilt es, die präklinische von der klinischen Therapie zu unterscheiden. Nach unverzüglicher EKG-Diagnostik vor Ort müssen bei STEMIPatienten sofort Erstmaßnahmen eingeleitet werden. Diese zielen zum einen auf eine Senkung des myokardialen Sauerstoffverbrauchs und zum anderen auf eine prähospitale Gerinnungshemmung im Sinne einer antithrombin- und antithrombozytären Therapie ab (Steg et al. 2012). Darüber hinaus werden in dieser Phase die Weichen der hospitalen Therapie gestellt, um eine schnellstmögliche Reperfusion zu ermöglichen.

Im klinischen Umfeld bieten sich zwei Reperfusionsverfahren an: die medikamentöse Lysetherapie sowie die primäre PCI. Nachdem die Lysetherapie viele Jahre als Goldstandard in der Therapie des STEMI galt, wurde sie in den letzten Jahren aufgrund der eindeutigen Studienlage von der primären PCI als Therapie der Wahl bei STEMI mit einer 
Symptomdauer $<12 \mathrm{~h}$ abgelöst, vorausgesetzt, diese ist ohne Zeitverzögerung (innerhalb von 120 min nach Diagnosestellung) möglich (Steg et al. 2012). Bei der PCI handelt es sich um ein mechanisches Verfahren zur Wiederherstellung des Blutflusses in der betroffenen Koronararterie. Das betroffene Gefäßsegment kann mit einem Koronardraht rekanalisiert und mit Hilfe eines Ballonkatheters aufgedehnt und das Gefäßlumen soweit vergrößert werden, dass die Unterbrechung des Blutflusses weitgehend aufgehoben wird (Lapp und Krakau 2013). Da bei alleiniger Ballondilatation eine erhöhte Rezidivrate besteht und zudem die Akutergebnisse schlechter sind, wird heute in den meisten Fällen nach vorheriger Ballondilatation ein Stent im betroffenen Gefäßsegment implantiert (Pinger 2010). Hierbei spielen medikamentenbeschichtete Stents (drug eluting stent) eine übergeordnete Rolle, da sie zu einer signifikanten Reduktion der Restenose- und Reinterventionsrate führen, allerdings nicht die Mortalität senken (Windecker et al. 2014). Um die Myokardschädigung möglichst gering zu halten und um die Prognose des Patienten positiv zu beeinflussen, sollte die PCI so schnell wie möglich erfolgen (Ziel: medizinischer Erstkontakt bis zur primären PCI $<90 \mathrm{~min}$ ). Um dies zu gewährleisten, ist eine Versorgung in Infarktnetzen mit geschultem Personal und entsprechender Logistik unabdingbar. Mittels einer schnellen Diagnostik am Notfallort sowie einer zeitnahen telefonischen Ankündigung kann bei einem STEMI-Patienten schon direkt nach Ankunft in der PCI-Klinik mit der Reperfusion begonnen werden (Steg et al. 2012). Je kürzer die door-to-balloon-Zeit (Zeit vom Eintreffen des Patienten in der Klinik bis zur ersten Balloninflation) ist, desto geringer ist die Sterblichkeit (Berger et al. 1999).

In diversen randomisierten Studien konnte die Überlegenheit der primären PCI im Vergleich zur Lysetherapie herausgestellt werden. Diese zeigte sich sogar auch dann, wenn der Patient zunächst aus einem Krankenhaus ohne PCI-Versorgung in ein PCI-Zentrum verlegt werden musste (O’Neill et al. 1986; Grines et al. 1993; Zijlstra et al. 1993; Betriu et al. 1997; Vermeer et al. 1999; Grines et al. 2002; Andersen et al. 2003; Widimský et al. 2003). Zu den großen Vorteilen der primären PCI zählt vor allem eine deutliche Senkung der Infarktsterblichkeit im Vergleich zur Lysetherapie sowohl im Kurz- als auch im Langzeitverlauf. In Meta-Analysen konnte darüber hinaus bei der PCI im Vergleich zur Lyse eine Reduktion der Schlaganfallrate aufgrund eines geringeren Hirnblutungsrisikos sowie ein geringeres Re-Infarktrisiko belegt werden (Weaver et al. 1997; Nunn et al. 1999; Zijlstra et al. 1999; Grines et al. 2003; Keeley et al. 2003). Auch funktionell lässt sich ein weiterer positiver Aspekt der PCI feststellen: So wird im Rahmen einer PCI deutlich häufiger ein TIMI-III-Fluss im Infarktgefäß (> $90 \%$ der Patienten) erreicht (Waldecker et al. 1997; Stone et al. 2002) als es im Vergleich zur reinen Lysetherapie (55 \% der Patienten) der Fall ist (Ross et al. 1993; Pinger 2010; Lapp und Krakau 2013). 
Leitliniengerecht kommt die medikamentöse Lysetherapie erst dann in Frage, wenn der Patient nicht innerhalb von $2 \mathrm{~h}$ nach Diagnosestellung einer PCI-Therapie zugeführt werden kann. Aufgrund der annähernd flächendeckenden PCI-Versorgung wird die Lysetherapie heutzutage nur noch selten eingesetzt. Eine weitere Möglichkeit der Revaskularisierung besteht in der Koronaren-Bypass-Chirurgie. Diese Option wird im Akutfall, u. a. aufgrund des hohen prozeduralen Risikos und der häufig nicht zeitnahen Verfügbarkeit, allerdings nur sehr begrenzt, z. B. bei fehlgeschlagener PCI, interventionell nicht beherrschbarem Koronarbefund sowie bei Komplikationen, eingesetzt (Steg et al. 2012).

\subsubsection{STEMI und Mehrgefäßerkrankung}

Eine besondere Risikogruppe stellen STEMI-Patienten dar, die neben dem akuten Gefäßverschluss weitere, höhergradige Stenosen in einem zusätzlichen Koronargefäß haben. Im Rahmen der Koronarangiographie beim akuten Myokardinfarkt wird bei bis zu 40-50 \% der untersuchten Patienten neben dem Infarktgefäß eine weitere relevante Stenose in einem Nicht-Infarkt-Gefäß im Sinne einer Mehrgefäßerkrankung (MVD = multivessel disease) diagnostiziert (Grines et al. 1999; Moreno et al. 2001). Das Outcome dieser Patienten ist im Vergleich zu Patienten mit Eingefäßerkrankung (SVD = singlevessel disease) erheblich schlechter. So ist zum einen der Revaskularisierungserfolg vermindert, zum anderen geht eine Mehrgefäßerkrankung im Rahmen eines akuten Myokardinfarktes nach PCIBehandlung des Infarktgefäßes mit einem reduzierten Kurz- und Langzeitüberleben einher (Sorajja et al. 2007). Im Hinblick auf eine optimale Behandlung dieser Risikopatienten zeigt sich aktuell eine kontroverse Studienlage (Cavender et al. 2009; Wald et al. 2013). Gemäß Leitlinie sollte sich beim akuten Myokardinfarkt die primäre PCI auf das Infarktgefäß beschränken, nur im Falle eines kardiogenen Schocks oder anhaltender Ischämie nach PCI sollte eine Mehrgefäß-PCI erwogen werden. Bei hämodynamisch stabilen STEMIPatienten mit Mehrgefäßerkrankung wird ein zweizeitiges Vorgehen zur Behandlung weiterer relevanter Stenosen empfohlen (Steg et al. 2012). Nichtsdestoweniger spielt eine mögliche Mehrgefäßerkrankung mit Blick auf die Risikostratifikation, Prognose und Therapie des STEMI-Patienten eine große Rolle; vom Vorteil ist hierbei, dass dieser Risikofaktor im Rahmen der primären PCI direkt erfasst wird.

\subsubsection{STEMI mit zusätzlich chronisch verschlossenem Nicht-Infarkt-Gefäß}

In die Gruppe der Patienten mit STEMI und MVD fallen auch solche, die zusätzlich zum akuten Gefäßverschluss noch einen weiteren, chronischen Totalverschluss in einem NichtInfarkt-Gefäß $\left(\mathrm{CTO}_{\text {NIRA }}\right)$ haben. Bei Patienten mit akutem STEMI und PCI-Behandlung wird die Häufigkeit eines CTO $_{\text {NIRA }}$ mit 8-13 \% (Van der Schaaf et al. 2006; Claessen et al. 
2009; Lexis et al. 2011; Bataille et al. 2012b; Claessen et al. 2012; Ariza-Solé et al. 2014) angegeben. Bei ca. 50 \% der Patienten befindet sich der CTO $_{\text {NIRA }}$ in der RCA (Tabelle 1).

Tabelle 1: $\mathrm{CTO}_{\text {NIRA-Lokalisation im Vergleich }}$

\begin{tabular}{|l|c|c|c|c|}
\hline & $\begin{array}{l}\text { Häufigkeit CTO } \\
\text { STEMIRA bei }\end{array}$ & $\begin{array}{l}\text { CTO }_{\text {NIRA }} \\
\text { in RCA }\end{array}$ & $\begin{array}{l}\text { CTO }_{\text {NIRA }} \\
\text { in RIVA }\end{array}$ & $\begin{array}{l}\text { CTO }_{\text {NIRA }} \\
\text { in RCX }\end{array}$ \\
\hline Bataille et al. (2012b) & $162 / 2020(8,0 \%)$ & $53 \%$ & $23 \%$ & $24 \%$ \\
\hline Lexis et al. (2011) & $90 / 1071(8,4 \%)$ & $43 \%$ & $32 \%$ & $24 \%$ \\
\hline Claessen et al. (2012) & $\begin{array}{c}283 / 3283(8,6 \%) \text { inkl. } 54 \text { Pat. } \\
\text { mit zwei CTO }\end{array}$ & $52 \%$ & $33 \%$ & $34 \%$ \\
\hline
\end{tabular}

Verschiedene Arbeitsgruppen stellten sich im Rahmen retrospektiver Kohortenstudien vor allem der Frage, ob die schlechte Prognose bei STEMI-Patienten mit MVD in einem zusätzlich chronisch verschlossenem Nicht-Infarkt-Gefäß begründet liegt. Ein Team aus Amsterdam kam zu dem Resultat, dass die hohe 30-Tage-, 1- und 3-Jahres-Sterblichkeit bei Patienten mit MVD maßgeblich von der Existenz eines $\mathrm{CTO}_{\text {NIRA }}$ abhängig ist (Claessen et al. 2012). Weitere Studien aus den Niederlanden und Polen stützen diese Resultate. Auch hier kam man zu dem Ergebnis, dass ein $\mathrm{CTO}_{\text {NIRA }}$ ein unabhängiger Risikofaktor für die Langzeitsterblichkeit (Lexis et al. 2011) bzw. für beides, Kurz- und Langzeitsterblichkeit ist (Tajstra et al. 2012). Hingegen zeigten Bataille et al. (2012b) in einer multivariaten Analyse, dass die Langzeitsterblichkeit bei STEMI vor allem mit einer MVD, der linksventrikulären Ejektionsfraktion (LVEF) und der Nierenfunktion assoziiert ist, nicht aber mit einem $\mathrm{CTO}_{\text {NIRA }}$ an sich. Eine Studie aus dem Vereinigten Königreich kam zu dem Ergebnis, dass ein $\mathrm{CTO}_{\text {NIRA }}$ zwar einen unabhängigen Einfluss auf die 30-Tage-Sterblichkeit, nicht aber auf die Langzeitsterblichkeit hat (Mozid et al. 2014). Die Ergebnisse all dieser Studien scheinen allerdings sehr davon abzuhängen, welche Variablen in den jeweiligen multivariaten Analysen berücksichtigt wurden (Bataille et al. 2012b).

Trotz dieser Kontroversen scheint der $\mathrm{CTO}_{\text {NIRA }}$ die Schlüsselrolle im schlechten Outcome von Patienten mit STEMI und MVD einzunehmen. Einer Meta-Analyse aller relevanten Studien zum Thema STEMI mit CTO $_{\text {NIRA }}$ zufolge liegt das absolute Langzeitsterblichkeitsrisiko bei STEMI-Patienten mit $\mathrm{CTO}_{\text {NIRA }}$ vs. Patienten ohne $\mathrm{CTO}_{\mathrm{NIRA}}$ bei 23,7 \% bzw. 9,0 \%. Dabei ist der Einfluss auf die Sterblichkeit vor allem im ersten Monat nach STEMI am stärksten ausgeprägt (O’Connor et al. 2015). Es konnte gezeigt werden, dass ein $\mathrm{CTO}_{\text {NIRA }}$ bei STEMI-Patienten mit einer schlechteren LV-Funktion (Claessen et al. 2009) und mit schlechteren Reperfusionsmarkern (z. B. geringerer Anteil TIMI-III-Fluss nach PCI) einhergeht (Lexis et al. 2011; Tajstra et al. 2012). Zudem präsentieren sich STEMI- 
Patienten mit $\mathrm{CTO}_{\mathrm{NIRA}}$ häufiger in der Hochrisikosituation eines kardiogenen Schocks (Bataille et al. 2012a).

Mit Blick auf eine angestrebte Behandlung des $\mathrm{CTO}_{\text {NIRA }}$ sind die Mortalitätsergebnisse der genannten Studien von großer Bedeutung. Eine frühzeitige therapeutische Intervention könnte möglicherweise, im Kontext der erhöhten Kurzzeitsterblichkeit, einen großen Einfluss auf die Prognose der STEMI-Patienten mit CTO $_{\text {NIRA }}$ haben (O'Connor et al. 2015). Zur Zeit ist aber noch unklar, wie genau diese Therapie auszusehen hat und wann sie erfolgt, z. B. direkt während der primären STEMI-PCI oder etappenweise, also im Anschluss an die Akutphase. Die erst kürzlich publizierte EXPLORE-Studie konnte keinen Vorteil einer zusätzlichen $\mathrm{CTO}_{\mathrm{NIRA}^{-}} \mathrm{PCI}$ innerhalb einer Woche nach der primären STEMIPCI gegenüber einem konservativen Vorgehen im Hinblick auf den CTO $_{\text {NIRA nach }}$ primärer STEMI-PCI präsentieren (Henriques et al. 2016).

Unabhängig von der Frage des Zeitpunkts der CTO-Intervention sollte die Indikation zur immer noch komplexen PCI-Therapie des $\mathrm{CTO}_{\text {NIRA }}$ ganz maßgeblich vom individuellen Risiko eines jeden Patienten abhängen. Ein Hauptaugenmerk sollte dabei auf dem individuellen koronarangiographischen CTO-Befund liegen. Bisher existieren diesbezüglich für das Patientenkollektiv STEMI mit $\mathrm{CTO}_{\mathrm{NIRA}}$ allerdings nur wenige Daten. Lexis et al. (2011) erfassten zwar die vom CTO betroffenen Koronargefäßsegmente bei STEMIPatienten, analysierten diese aber nicht hinsichtlich der Sterblichkeitsprognose, sondern lediglich im Zusammenhang mit pathologischen Q-Zacken, auch fand der Koronarversorgungstyp keine Berücksichtigung. In einer unlängst veröffentlichten Studie aus den Niederlanden (Hoebers et al. 2016) wurde der Einfluss der Lokalisation des CTO $_{\text {NIRA }}$ bei STEMI-Patienten erstmals detaillierter untersucht. Aufgrund der thematischen Überschneidung mit der vorliegenden Untersuchung werden die Ergebnisse dieser Studie im Diskussionsteil präsentiert und erörtert. Zwei weitere Studiengruppen beschäftigten sich zudem ausgiebiger mit der Kollateralversorgung bei Patienten mit STEMI und CTO NIRA $_{\text {ST }}$ (Bataille et al. 2013; Şen et al. 2016). Die präsentierten Ergebnisse widersprechen sich allerdings teilweise und werden ebenfalls im Rahmen der Diskussion detailliert aufgegriffen. 


\section{Fragestellung}

Das Thema CTO steht derzeit im Fokus, da in den vergangenen Jahren Techniken entwickelt werden konnten, die es möglich machen, auch chronische Koronarverschlüsse mit hoher Erfolgsquote katheter-interventionell zu rekanalisieren. Dies führte bei Patienten mit KHK zu einer Ausweitung der Indikationsstellung zur PCI als Alternative zur Koronarbypass-OP. Aus früheren Arbeiten gibt es Hinweise, dass Patienten mit akutem STEMI bei Vorliegen eines zusätzlichen chronischen Verschlusses in einem Nicht-InfarktGefäß $\left(\mathrm{CTO}_{\text {NIRA }}\right)$ eine schlechtere Prognose haben. Allerdings liegen hierzu bisher nur wenige Daten vor.

Ziel der vorliegenden Arbeit war es, bei STEMI-Patienten die prognostische Bedeutung eines zusätzlichen $\mathrm{CTO}_{\mathrm{NIRA}}$ detailliert zu analysieren. Im Einzelnen sollten dabei folgende Fragen geklärt werden:

1. Wie häufig liegt bei Patienten mit akutem STEMI ein zusätzlich chronischer Verschluss in einem Nicht-Infarkt-Gefäß $\left(\mathrm{CTO}_{\text {NIRA }}\right)$ vor?

2. Welche Gefäße sind vom $\mathrm{CTO}_{\text {NIRA }}$ besonders betroffen und wo im Gefäß sind die chronischen Verschlüsse vorwiegend lokalisiert?

3. Wie stark beeinflusst bei STEMI ein $\mathrm{CTO}_{\text {NIRA }}$ die Prognose?

4. Welche prognostische Rolle spielt dabei der exakte angiographische Befund (Lokalisation des CTO, Koronarversorgungstyp und Kollateralstatus)?

5. Welche prognostische Bedeutung haben anamnestische bzw. elektrokardiographische Hinweise auf einen bereits früher abgelaufenen Infarkt?

6. Welche Faktoren und Pathomechanismen bestimmen bei STEMI mit $\mathrm{CTO}_{\mathrm{NIRA}}$ die Sterblichkeit? 


\section{Patienten und Methoden}

\subsection{Patientenkollektiv}

In dieser retrospektiven Analyse wurden initial prospektiv in einem Zeitraum über sechs Jahre vom 01.03.2008 bis zum 28.02.2014 alle Patienten unselektiert eingeschlossen, die mit der Diagnose eines STEMI und dem Ziel einer primären PCI in die Universitätsmedizin Göttingen (UMG) aufgenommen wurden. Das Kollektiv beinhaltet somit auch Patienten, die trotz der eigentlichen Therapieintention aus unterschiedlichen Gründen keine Koronarangiographie erhielten oder nicht im Rahmen einer primären Koronarintervention behandelt wurden. Darüber hinaus wurden auch Patienten erfasst, deren Infarktsymptomatik während eines stationären Aufenthaltes in der UMG auftrat $(\mathrm{IIH}=$ Infarkt Intrahospital).

Als Einschlusskriterium galt neben der typischen Infarktsymptomatik mit einer Symptomdauer von $<24 \mathrm{~h}$ bis zum ersten medizinischen Kontakt eine leitliniengerechte EKG STEMI-Diagnose (Steg et al. 2012):

- ST-Strecken-Hebung in zwei zusammenhängenden Ableitungen $\geq 0,1 \mathrm{mV}$ bzw. in den Ableitungen $V_{2}$ und $V_{3}$ bei Männern $<40$ Jahren $\geq 0,25 \mathrm{mV}$, bei Männern $>40$ Jahren $\geq 0,2 \mathrm{mV}$ und bei Frauen $\geq 0,15 \mathrm{mV}$.

- atypische EKG-Veränderungen bei einem neu aufgetretenen Linksschenkelblock.

\subsection{Aus der Analyse ausgeschlossene Patienten}

STEMI-Patienten mit Zustand nach Koronararterienbypass-Operation (CABG = coronary artery bypass graft) wurden in der Datenbank zwar erfasst, sie wurden aber trotz einer hohen Prävalenz an chronischen Verschlüssen für die Analyse hinsichtlich der Fragestellung der vorliegenden Arbeit exkludiert. Grund für den Ausschluss ist die antegrade Perfusion des Gefäß-Areals distal eines CTO durch einen funktionierenden Koronarbypass. Somit liegt in diesen Fällen funktionell keine relevante Verschlusskonstellation vor. Zudem wird möglicherweise bei Patienten mit Koronarbypass auch das Ausmaß einer Kollateralisierung entscheidend verändert. Darüber hinaus könnte aufgrund des hohen Sterblichkeitsrisikos dieser Patienten mit CABG (Stone et al. 2000) die prognostische Relevanz eines chronischen Verschlusses mutmaßlich verfälscht werden.

\subsection{Einteilung nach Patientengruppen}

Die eingeschlossenen STEMI-Patienten wurden anhand des koronarangiographischen Befundes bezüglich des Gefäßerkrankungstyps in verschiedene Gruppen eingeteilt. 
Berücksichtigt wurden hierbei das akute Infarktgefäß sowie alle weiteren von einer relevanten Stenosierung $>50$ \% betroffenen epikardialen Gefäße (RCX, RIVA und RCA). Patienten, die trotz der Diagnose STEMI in der Koronarangiographie kein relevant stenosiertes Koronargefäß aufwiesen, wurden der Gruppe ZVD (zerovessel disease; Nullgefäßerkrankung) zugewiesen. Patienten mit einer 1-Gefäßerkrankung hatten neben dem akuten Verschluss keine weiteren relevanten Stenosen der übrigen Koronararterien, diese wurden der Gruppe SVD (singlevessel disease; Eingefäßerkrankung) zugeordnet. Alle Patienten mit 2- oder 3-Gefäßerkrankung sowie Patienten mit Hauptstammstenose wurden in die Gruppe MVD (multivessel disease; Mehrgefäßerkrankung) eingeteilt. Diese Gruppe MVD wurde, je nachdem ob in einem Nicht-Infarkt-Gefäß (noninfarct-related artery) ein zusätzlich chronischer Verschluss $\left(\mathrm{CTO}_{\text {NIRA }}\right)$ vorlag, weiter unterteilt. So gab es eine Gruppe von Patienten mit Mehrgefäßerkrankung, aber fehlendem zusätzlich chronischen Verschluss (MVD -CTO ${ }_{\text {NIRA }}$ ), und eine Gruppe von Patienten mit Mehrgefäßerkrankung und zusätzlich chronischem Verschluss $\left(\mathrm{MVD}+\mathrm{CTO}_{\text {NIRA }}\right)$. Letztere konnte zudem in eine Gruppe mit Mehrgefäßerkrankung und ausschließlich einem vorhandenen zusätzlich chronischen Verschluss (MVD $+1 \mathrm{CTO}_{\mathrm{NIRA}}$ ) sowie in eine Gruppe mit Mehrgefäßerkrankung und mehreren chronischen Verschlüssen (MVD +>1CTO NIRA $_{2}$ unterteilt werden. Patienten, die trotz STEMI Diagnose keine akute Koronarangiographie erhielten und somit nicht in die oben genannten, auf dem Koronarbefund basierenden Gruppen eingeteilt werden konnten, wurden gesondert der Gruppe „keine Koronarangiographie“ zugeordnet.

\subsection{Datenerfassung}

Alle in dieser Arbeit eingeschlossenen STEMI-Patienten wurden in der Universitätsmedizin Göttingen im Rahmen des FITT-STEMI (Feedback-Intervention and Treatment-Times in ST-Elevation Myocardial Infarction) -Projektes prospektiv erfasst (Scholz et al. 2012). Hierbei handelt es sich um ein multizentrisches Qualitätsmanagement-InterventionsProjekt zur Prozessoptimierung bei der Versorgung von Patienten mit akutem STEMI. Das Hauptaugenmerk dieses Forschungsunternehmens liegt auf der Erfassung und Rückkopplung der wesentlichen Behandlungszeiten bei STEMI-Patienten. Die Universitätsmedizin Göttingen ist seit dem 01.03.2008 als eine von sechs Pilotphase-Kliniken in dieses Projekt integriert. Von Seiten der Ethik-Kommission der Medizinischen Fakultät der Universität Göttingen lagen laut einer zustimmenden Bewertung im Zuge einer Sitzung vom 20.09.2007 keine rechtlichen oder ethischen Bedenken gegen die Durchführung dieses Forschungsvorhabens vor (Antragsnummer: 1/10/07). 
Zentrales Element der FITT-STEMI-Datenerfassung ist ein standardisierter Dokumentationsbogen (siehe Anhang, Kapitel 7.1, Abbildung 46 und 47). Dieser Bogen war somit auch Grundlage der Datenerfassung dieser Promotionsarbeit. Die Datenerfassung anhand des Dokumentationsbogens erfolgte bereits während der Behandlung des Patienten im Herzkatheterlabor. Jeder STEMI-Patient wurde im Rahmen des FITT-STEMIProjektes mit einer eigenen Fallnummer für die Web-Erfassung pseudonymisiert. Die einzelnen Intervalle der Behandlungszeiten im Rahmen der Koronarintervention wurden dabei digital gespeichert und vom Herzkatheterpersonal in den Dokumentationsbogen übertragen. Im Anschluss an den primären Eingriff fügte der behandelnde Kardiologe weitere angiographische Einzelheiten zum Procedere, wie z. B. den Gefäßerkrankungstyp, das rekanalisierte Gefäß und den TIMI Grade Flow Vor- und Nach-PCI, dem Dokumentationsbogen hinzu.

Im Verlauf des Krankenhausaufenthaltes erfolgte dann eine normierte Anamneseerhebung in der, soweit es der Zustand des Patienten ermöglichte, gezielt einzelne Patientencharakteristika erfragt wurden. Ergänzend konnten relevante Informationen auch aus der Patientenakte bzw. aus einer Fremdanamnese in Erfahrung gebracht werden. Aus den vorliegenden Rettungsdienstprotokollen wurden die Einsatzzeiten und weitere rettungstechnische Daten dokumentiert, bei Unvollständigkeit waren diese zudem über die zuständige Rettungsleitstelle greifbar. In der Zusammenschau mit den Zeiten der Herzkatheterversorgung entstand so ein lückenloses Zeitprofil jedes Patienten, vom Symptombeginn bis zur erfolgreichen Rekanalisierung im PCI-Krankenhaus.

Zur Archivierung der einzelnen Patientenfälle wurden FITT-STEMI-Projektordner mit allen für das Projekt bedeutenden Dokumenten (Dokumentationsbogen, prähospitales EKG, Einweisungs- und Überweisungspapiere, Rettungsdienstprotokolle, Herzkatheterund weitere Befundprotokolle sowie Entlassungsbriefe) angelegt. In einem weiteren Schritt konnten die Daten dann pseudonymisiert in die web-basierte Datenbank übertragen werden, diese wurden dort zudem direkt automatisiert auf Vollständigkeit überprüft. Darüber hinaus fand auch eine stichprobenartige Überprüfung der Datenvalidität durch ein unabhängiges Monitoring des IFS (Institut für anwendungsorientierte Forschung und klinische Studien) statt.

Die so erfassten Patienten wurden nach ausgiebiger Information und unterzeichneter Einverständniserklärung zudem über den akuten Verlauf hinweg über einen längeren Zeitraum begleitet, um ein möglichst vollständiges 30-Tage- und 1-Jahres-Follow-upErgebnis zu erhalten. Zur Analyse konnten alle online erfassten Daten dann in Form einer Microsoft ${ }^{\circledR}$ Excel® Pivot Tabelle heruntergeladen und statistisch ausgewertet werden. 
Da zum Zeitpunkt der Aufnahme dieser Promotionsarbeit angiographische Merkmale wie „CTO ${ }_{\text {NIRA }}$ “ oder „Kollateralisierung“ nicht im FITT-STEMI-Dokumentationsbogen erfasst werden konnten, wurden diese Daten retrospektiv nach den unten stehenden Kriterien aus den vorliegenden angiographischen Filmaufnahmen und Befunden dokumentiert. Zudem wurden die archivierten prähospitalen EKGs der STEMI-Patienten mit $\mathrm{CTO}_{\mathrm{NIRA}}$ auf pathologische Q-Zacken untersucht. All diese Ergebnisse wurden dann eigenhändig mit den online verfügbaren Daten verknüpft und analysiert.

\subsection{Erfassung angiographischer Merkmale}

Die in Tabelle 2 präsentierten koronarangiographischen bzw. -interventionellen Patientenmerkmale wurden bereits prospektiv im Rahmen des FITT-STEMIDokumentationsbogens registriert.

Tabelle 2: Im Dokumentationsbogen erfasste angiographische Merkmale

\begin{tabular}{|l|l|}
\hline \multicolumn{1}{|c|}{ Merkmal } & \multicolumn{1}{|c|}{ Auswahlmöglichkeit } \\
\hline Gefäßerkrankungstyp & $\begin{array}{l}\text { 0-Gefäß-, 1-Gefäß-, 2-Gefäß- und 3-Gefäß-KHK sowie } \\
\text { Hauptstammstenose }\end{array}$ \\
\hline Rekanalisiertes Gefäß & RIVA, RCA, RCX, linker Hauptstamm sowie Bypass-Graft \\
\hline TIMI Grade Flow & 0-III, jeweils Vor- und Nach-PCI \\
\hline $\begin{array}{l}\text { Gründe für nicht erfolgte } \\
\text { Koronarangiographie }\end{array}$ & $\begin{array}{l}\text { Reanimation, Punktion nicht möglich, EKG-Normalisierung, } \\
\text { STEMI nicht erkannt, Tumorleiden, Apoplex, Alter/AZ, ,andere“ }\end{array}$ \\
\hline $\begin{array}{l}\text { Gründe für nicht erfolgte } \\
\text { PCI }\end{array}$ & $\begin{array}{l}\text { Myokarditis, Tako-Tsubo-Kardiomyopathie, kein Zielgefäß, } \\
\text { zusätzlich bestehende LCA-Hauptstammstenose, Akut-Bypass-OP, } \\
\text { Vasospasmus, Drahtpassage nicht möglich, ,andere“ }\end{array}$ \\
\hline
\end{tabular}

Die angiographischen Schlüsselmerkmale für diese Arbeit $\left(\mathrm{CTO}_{\mathrm{NIRA}}\right.$, Kollateralisierung und einheitlich definierter Koronarversorgungstyp) wurden nicht im Rahmen der FITTSTEMI-Studie erfasst und mussten somit retrospektiv im Abgleich mit den im klinischen Alltag verfassten Herzkatheterprotokollen und -befundberichten registriert werden. Da ein vollständiger Untersuchungsbericht des interventionellen Kardiologen die komplette Koronaranatomie und -morphologie enthält, wurden hierzu in einer ersten Sitzung alle angiographischen Befunde der FITT-STEMI-Projektordner aller STEMI-Patienten des betroffenen Zeitraumes nach Hinweisen auf chronische Verschlüsse durchsucht. Nach Selektion aller als „chronischer Verschluss in einem Nicht-Infarkt-Gefäß $\left(\mathrm{CTO}_{\text {NIRA }}\right)$ “ sowie auch aller als „subtotal verschlossenes Nicht-Infarkt-Gefäß“ bewerteten Befunde wurden die Koronarangiogramme all dieser Patienten systematisch durchgesehen und nach 
einheitlicher Definition auf das Vorliegen eines tatsächlichen CTO $_{\text {NIRA }}$ beurteilt. Auf diese Art und Weise konnte für jeden STEMI-Patienten mit $\mathrm{CTO}_{\text {NIRA }}$ der komplette Koronarstatus inklusive $\mathrm{CTO}_{\mathrm{NIRA}}$-Segment, Koronarversorgungstyp und Kollateralstatus definitionsgemäß erhoben werden. Im Anschluss daran wurden die erhobenen angiographischen Merkmale mit den prospektiv über den FITT-STEMI-Dokumentationsbogen erfassten Daten verknüpft und schließlich analysiert.

Ein Verschluss wurde hierbei als $\mathrm{CTO}_{\mathrm{NIRA}}$ charakterisiert, wenn in einem nicht vom akuten Infarkt betroffenen verschlossenen Gefäß ein TIMI-0-Fluss vorherrschte und dieser Verschluss mutmaßlich seit mindestens drei Monaten bestand (Sianos et al. 2012). Bedingt durch extraluminale Brückenkollateralen kann allerdings im angiographischen Bild selbst bei einem TIMI-Fluss von 0 über dem betroffenen Segment ein anterograder Fluss ermittelt werden, was die Diagnosestellung erschwerte. Zudem war die genaue Bestimmung der Verschlussdauer schwierig, sie stützte sich, sofern kein angiographischer Beweis auf Grundlage älterer Befunde vorlag, meist auf Schätzungen, die sich aus angiographischen (Verschlussmorphologie: z. B. stark verkalkte Läsionen mit ausgeprägter Kollateralisierung) oder klinischen Informationen (Q-Zacken-EKG im betroffenen Infarktareal, Myokardinfarkt in der Anamnese) ergaben.

Zur einheitlichen Bestimmung von Versorgungstyp und $\mathrm{CTO}_{\text {NIRA }}$-Lokalisation wurde in dieser Arbeit die Koronarsegmentdefinition zur Ermittlung des SYNTAX-Scores (Abbildung 1) übernommen (Sianos et al. 2005). Diese Einteilung in 16 verschiedene Koronarsegmente basiert zum Großteil auf der Klassifikation des AHA Grading Committees aus dem Jahr 1975 (Austen et al. 1975). Beachtenswert ist hinsichtlich der Koronarversorgung, dass bei der Ermittlung des SYNTAX-Scores und somit auch in dieser Arbeit lediglich zwischen Links- und Rechtsversorgertyp unterschieden wurde. Ein potentieller kodominanter Versorgungstyp wurde dem Rechtsversorgungstyp zugeordnet, da in beiden Fällen der linke Ventrikel sowohl über die LCA als auch über die RCA versorgt wird. Zudem konnte in einigen Fällen nicht zweifelsfrei zwischen Rechts- und KoDominanz unterschieden werden, da die betroffenen Gefäße aufgrund des chronischen Verschlusses nicht immer vollständig dargestellt wurden. Die exakte Definition des einzelnen Versorgungstyps ist in Kapitel 1.2 dargelegt.

Zusätzlich zur genauen Segmentklassifikation wurden die vom CTO $_{\text {NIRA }}$ betroffenen Segmente abhängig von der Entfernung zum Ostium in eine proximale (Segment: 1, 5, 6 und 11), mediale (Segment: 2 und 7), distale (Segment: 3, 8 und 13) sowie periphere (Segment 4, 9, 10, 12, 14, 14a, 14b, 15 und 16) Gruppe eingeteilt, um der Größe und der Relevanz des distal des Verschlusses gelegenen Myokardareals gerecht zu werden. Im Falle eines Linksversorgungstyps wäre ein $\mathrm{CTO}_{\text {NIRA }}$ in Segment 13 des RCX als CTO eines 
medialen Segments gewertet worden, allerdings trat dieser Fall in der vorliegenden Untersuchung nicht ein.

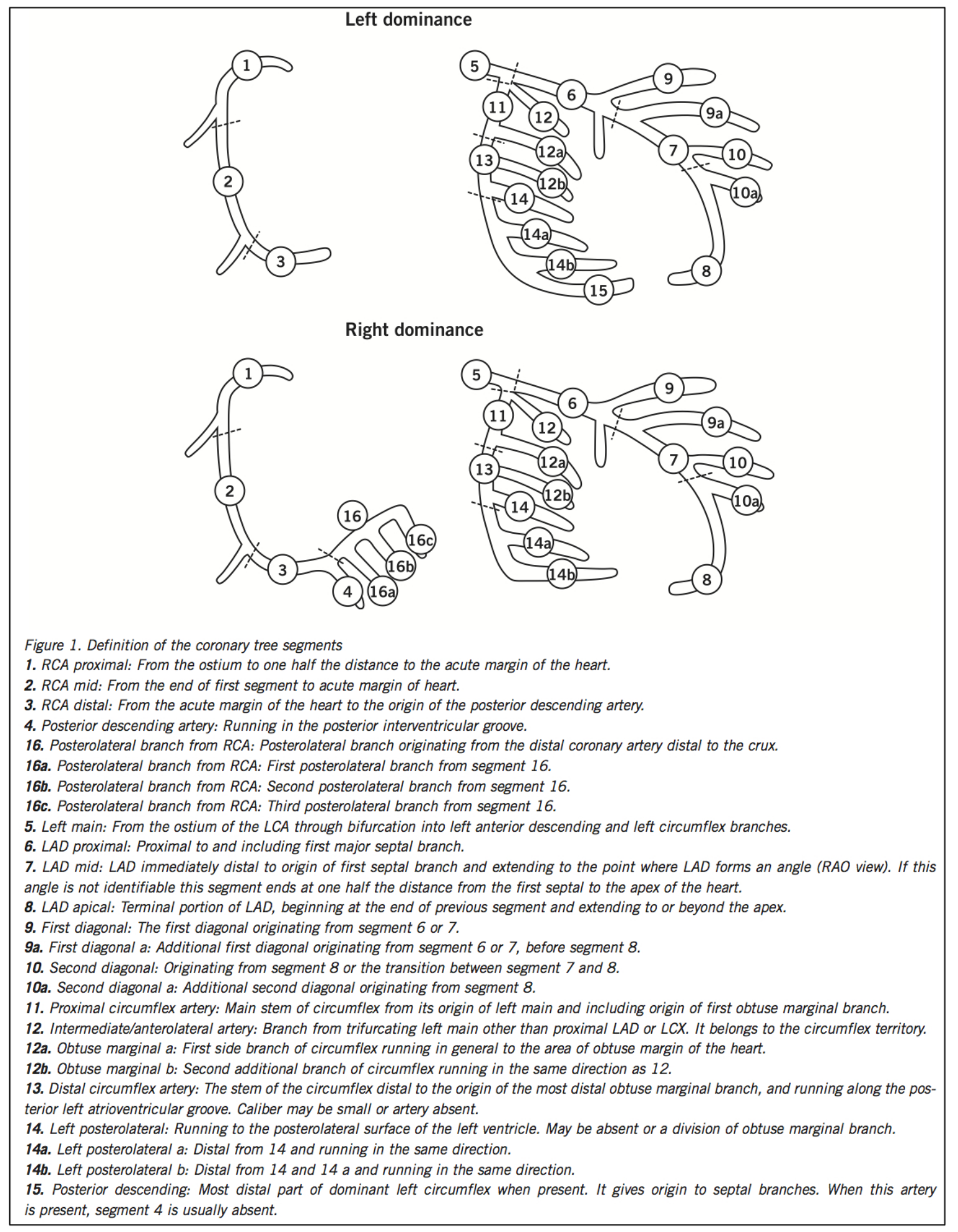

Abbildung 1: Segmentdefinition nach: The SYNTAX Score (Sianos et al. 2005); Nachdruck aus (reprinted from) EuroIntervention Vol. 1 (2), Authors: Sianos et al., Title: The SYNTAX Score: an angiographic tool grading the complexity of coronary artery disease. Pages: 219_ 227, Copyright (2005), mit freundlicher Genehmigung von (with permission from) Europa Digital \& Publishing. 
Der Kollateralgefäßstatus wurde, wenn durchgeführt, sowohl vor als auch nach der primären PCI beurteilt, um auch solche Kollateralen zu detektieren, die ihren Ursprung im akuten Infarktgefäß distal der Verschlussstelle haben. Als Kollateralen zum betroffenen $\mathrm{CTO}_{\text {NIRA }}-\mathrm{Gefäß}$ wurde nur solche gewertet, die einen Rentrop Score von 2 oder 3 (teilweise bzw. komplette kollaterale Füllung des verschlossenen epikardialen Gefäßsegments) aufwiesen (Rentrop et al. 1985). Dabei wurden im Sinne des CC grade alle möglichen Klassifizierungen berücksichtigt: $\mathrm{CCO}=$ keine kontinuierliche Verbindung zwischen Spender- und Empfängerarterie; CC1 $=$ kontinuierliche, fadenförmige Verbindung; CC2 = kontinuierliche, seitastförmige Verbindung (Werner et al. 2003). Bei der anatomischen Betrachtung der Kollateralbahnen wurde im Rahmen dieser Arbeit zwischen Brückenkollateralen (alle intrakoronaren Verbindungen) und interarteriellen bzw. interkoronaren Kollateralen (septale und epikardiale Kollateralen zusammengefasst; vergleiche Kapitel 1.3.4) unterschieden. Interarterielle Kollateralverbindungen, hervorgehend aus dem vom akuten Infarkt betroffenen Gefäß, wurden zudem nach dem Ursprungsort der Kollaterale in proximal und distal des akuten Verschlusses unterteilt.

Ein angiographischer Beispielbefund mit allen wesentlichen hier genannten Merkmalen befindet sich im Anhang (siehe Kapitel 7.2, Abbildung 48 - 51).

\subsection{EKG-Analyse}

Die STEMI-Lokalisation im EKG wurde bereits prospektiv bei Aufnahme des Patienten im Dokumentationsbogen erfasst. Hierbei wurde zwischen folgenden Lokalisationen unterschieden: Vorderwandinfarkt, Lateralwandinfarkt, Hinterwandinfarkt sowie neu aufgetretener Linksschenkelblock. Im Rahmen der vorliegenden Untersuchung wurde das initiale STEMI-EKG aller erfassten Patienten mit $\mathrm{CTO}_{\mathrm{NIRA}}$ zudem retrospektiv auf die Zeichen eines abgelaufenen Infarktes in den zum $\mathrm{CTO}_{\text {NIRA }}-$ Gefäß passenden EKGAbleitungen im Sinne einer pathologischen Q-Zacke untersucht. Für einen alten abgelaufenen Q-Zacken-Infarkt sprechen nach der third universal definition of myocardial infarction folgende EKG-Kriterien (Thygesen et al. 2012):

- jede Q-Zacke in $\mathrm{V}_{2}-\mathrm{V}_{3} \geq 0,02$ s oder QS-Komplex in $\mathrm{V}_{2}$ und $\mathrm{V}_{\mathrm{r}}$.

- Q-Zacke $\geq 0,03$ s und $\geq 0,1 \mathrm{mV}$ tief oder QS-Komplex in I, II, aVL, aVF oder $\mathrm{V}_{4-6}$ in zwei aufeinanderfolgenden Ableitungen.

\subsection{Basischarakteristika}

Neben den bereits erwähnten rettungstechnischen und den behandlungsrelevanten Daten sowie den angiographischen Merkmalen und EKG-Befunden wurden auf Grundlage des 
FITT-STEMI-Dokumentationsbogens unter anderem folgende, für die vorliegende Untersuchung relevante, Patienteninformationen erfasst (Tabelle 3).

Tabelle 3: Im Dokumentationsbogen erfasste Patientencharakteristika

\begin{tabular}{|c|c|}
\hline Patientencharakteristikum & Auswahlmöglichkeit \\
\hline Zeitpunkt der Patientenaufnahme & Regulärdienst oder Rufbereitschaft \\
\hline Alter & Jahre \\
\hline Geschlecht & Weiblich/Männlich \\
\hline $\begin{array}{l}\text { Body Mass Index (BMI) (WHO } \\
\text { Consultation on Obesity 2000). }\end{array}$ & $\begin{array}{l}\text { Aus Patientengewicht und -größe ermittelt. } \\
\text { Gruppeneinteilung nach WHO. }\end{array}$ \\
\hline \multicolumn{2}{|l|}{ Postleitzahl am Einsatzort } \\
\hline Einzugsgebiet & PCI- oder Nicht-PCI-Klinik \\
\hline Thrombolyse & $\mathrm{Ja} / \mathrm{Nein}$ \\
\hline Hausarztkontakt & Nein/telefonisch/vor Ort/in Praxis \\
\hline Vormedikation & $\begin{array}{l}\text { ASS, ACE-Inhibitor, AT1-Inhibibitor, } \\
\text { Aldosteron-Antagonist, Diuretikum, Lipid- } \\
\text { senker, Beta-Blocker, Kalziumantagonist, } \\
\text { Clopidogrel, Nitrat, Marcumar, keine Angabe } \\
\text { möglich }\end{array}$ \\
\hline Kardiale Risikofaktoren & $\begin{array}{l}\text { Arterielle Hypertonie, DM, anamnestisch AP, } \\
\text { Hyperlipoproteinämie, Nikotin, positive } \\
\text { Familienanamnese }\end{array}$ \\
\hline Vorerkrankungen & $\begin{array}{l}\text { Niereninsuffizienz, pAVK, sowie Zustand } \\
\text { nach: Myokardinfarkt, PCI, Apoplex, CABG }\end{array}$ \\
\hline $\begin{array}{l}\text { Killip-Klassifikation (Killip und Kimball } \\
\text { 1967) }\end{array}$ & $\begin{array}{l}\text { I: keine Herzinsuffizienzzeichen; II: feinblasige } \\
\text { Rasselgeräusche, dritter Herzton oder Jugular- } \\
\text { venenstauung; III: Lungenödem; IV: kardio- } \\
\text { gener Schock }\end{array}$ \\
\hline Mechanische Reanimation präklinisch & $\mathrm{Ja} / \mathrm{Nein}$ \\
\hline Intraaortale Ballongegenpulsation & $\mathrm{Ja} / \mathrm{Nein}$ \\
\hline TIMI Risk Score (TRS) & Automatisch berechnet \\
\hline Grund für fehlende Direktübergabe HKL & $\begin{array}{l}\text { Rettungsdienst, Diagnose initial unklar, } \\
\text { Katheterplatz besetzt, CT prä-HKU, Personal } \\
\text { nicht anwesend, Primärversorgung aufwendig }\end{array}$ \\
\hline LV-Ejektionsfraktion & $(\%)$ \\
\hline Tod (intrahospital/innerhalb 30 Tagen) & $\mathrm{Ja} / \mathrm{Nein}$ \\
\hline NYHA-Klasse 30 Tage & $0-\mathrm{IV}$ \\
\hline
\end{tabular}




\subsection{Behandlungszeiten und rettungstechnische Daten}

Das Hauptaugenmerk der Datenakquisition im Rahmen des FITT-STEMI-Projekts liegt auf der Erfassung und Rückkopplung aller zeitlichen Abläufe der akuten Infarktversorgung. Diese spielten allerdings in der vorliegenden Arbeit eine untergeordnete Rolle. In der prähospitalen Phase wurden vom Symptombeginn beim Patienten, über den eingegangen Notruf, der Ankunft des Rettungsdienstes am Einsatzort und dem Zeitpunkt des ersten EKG bis zum Verlassen des Einsatzortes, alle Zeiten dokumentiert und erfasst.

Die hospitale Phase begann mit dem Eintreffen des Patienten über die Notaufnahme der UMG. Auch hier wurden alle wichtigen Zeiten in der Therapiekette, von der Ankunft in der Klinik, über die Ankunft des Patienten im Herzkatheterlabor bis zur arteriellen Punktion und schließlich der Balloninflation, registriert. Aus diesen Zeitpunkten wurden dann über die web-basierte Datenbank wichtige Zeitintervalle bestimmt. Von besonderer Relevanz waren hierbei die Prähospitalzeit (Intervall vom Symptombeginn bis zur Ankunft in der PCI-Klinik), die door-to-balloon-Zeit (Intervall vom Eintreffen in der PCI-Klinik bis zur ersten Balloninflation) sowie die contact-to-balloon-Zeit (C2B-Zeit = Intervall vom ersten medizinischen Kontakt bis zur ersten Balloninflation in der PCI-Klinik).

Nach der Art der Zuweisung in das PCI-Zentrum (Transportart) wurden die Patienten in unterschiedliche Gruppen eingeteilt. So wurde zwischen Primärtransporten (direkter Transport vom Einsatzort in das PCI-Zentrum), Sekundärtransporten (initiale Einweisung in eine Nicht-PCI-Klinik mit anschließender Verlegung in das PCI-Zentrum), Selbstvorsteller (selbstständige Vorstellung des Patienten in der Notaufnahme des PCIZentrums) sowie Intrahospitalinfarkte (Patienten, die den STEMI während eines Krankenhausaufenthaltes im PCI-Zentrum erlitten) unterschieden. Bei den Patienten mit Primär- und Sekundärtransport wurden zudem neben dem Zeitpunkt des ersten EKG eine erfolgte telefonische Infarktankündigung im Herzzentrum, ein potentielles TelemetrieEKG sowie eine erfolgte Direktübergabe durch das Rettungsteam im Herzkatheterlabor mit Umgehung der Notaufnahme als wichtige Bestandteile der Therapiekette erfasst.

\subsection{Statistik}

Die online erfassten Daten aus dem FITT-STEMI-Dokumentationsbogen wurden mit den im Rahmen dieser Untersuchung neu erhobenen Daten verknüpft und in Form einer Microsoft ${ }^{\circledR}$ Excel® Pivot Tabelle zur Verfügung gestellt. Die so abrufbaren Rohdaten konnten daraufhin statistisch deskriptiv analysiert werden. Zur statistischen Auswertung dieser Daten wurde die Software Statistica 64 (Version 12.7.207.0) der Firma Dell Inc. 
verwendet. Die statistische Analyse erfolgte in enger Zusammenarbeit mit dem Institut für Medizinische Statistik der Universitätsmedizin Göttingen (Direktor: Prof. Dr. Tim Friede).

Kategoriale Variablen (z. B. Nominal- und Ordinalskalenniveau) wurden als absolute Häufigkeiten und Prozente angegeben. Unterschiede zwischen den verschiedenen Gruppen wurden dabei mittels Pearson-Chi-Square Test auf eine statistische Signifikanz hin getestet. In diesem Rahmen wurde ein p-Wert $<0,05$ als statistisch signifikanter Zusammenhang gewertet.

Kontinuierliche Variablen wie z. B. das Alter, der BMI, die LV-Ejektionsfraktion sowie der TIMI Risk Score wurden im Mittelwert der einzelnen Untergruppen bestimmt, es wurde zudem die Standardabweichung angegeben. Waren diese Merkmale in den Gruppen normalverteilt, so konnten mit Hilfe des Zweistichproben-t-Tests mögliche Unterschiede zwischen den Gruppen auf eine Signifikanz getestet werden. Zeigte sich indes keine Normalverteilung, so wurde der Mann-Whitney-U-Test angewendet. Auch hier wurde ein p-Wert $<0,05$ als statistisch signifikanter Zusammenhang gewertet.

Bezüglich der ermittelten contact-to-balloon-Zeiten in den einzelnen Untergruppen wurde als Maß der zentralen Tendenz jeweils der Median bestimmt. Mögliche Unterschiede zwischen den Gruppen wurden ebenfalls mittels Mann-Whitney-U-Test auf eine Signifikanz geprüft. Um mögliche Prädiktoren der Krankenhaussterblichkeit in der Gruppe der STEMIPatienten mit $\mathrm{CTO}_{\text {NIRA }} \mathrm{zu}$ identifizieren, wurden relevante Konfounder der Krankenhaussterblichkeit (mit $\mathrm{p}<0,1$ ) eines univariaten Vortests in ein Logit-Modell (logistische Regression) zur multivariaten Analyse eingespeist. Die Ergebnisse dieses Logit-Modells wurden als Odds-Ratios präsentiert. Darüber hinaus wurden noch weitere Variablen ohne Vortest in ein Logit-Modell zur rückwärtsgerichteten schrittweisen (backward stepwise) Identifizierung signifikanter Konfounder der Krankenhaussterblichkeit eingefügt. In dieser Analyse wurden Schritt für Schritt unabhängige Variablen, beginnend mit der Variablen mit dem geringsten Zusammenhang zur abhängigen Variable, aus dem Modell entfernt, um im endgültigen Modell lediglich signifikante Konfounder der Krankenhaussterblichkeit zu präsentieren. 


\section{Ergebnisse}

\subsection{Patienten}

\subsubsection{Eingeschlossene Patienten}

Im Studienzeitraum vom 01.03.2008 bis zum 28.02.2014 wurden über sechs Jahre insgesamt 1.303 Patienten mit der Diagnose eines akuten ST-Strecken-Hebungsinfarktes und dem Ziel einer katheter-interventionellen Therapie in der Universitätsmedizin Göttingen behandelt. Bei 56 dieser STEMI-Patienten bestand ein Zustand nach Koronarbypass-OP, diese Patienten wurden daher in der vorliegenden Untersuchung von der Analyse ausgeschlossen (siehe Kapitel 3.2).

Insgesamt wurden somit 1.247 STEMI-Patienten in die Endauswertung dieser Arbeit genommen. Diese Patienten wurden anhand des koronarangiographischen Befundes bezüglich des Gefäßerkrankungstyps in verschiedene Gruppen unterteilt (siehe Kapitel 3.3):

Bei fünf STEMI-Patienten (0,4\%) wurde aus unterschiedlichen Gründen keine AkutKoronarangiographie durchgeführt, somit konnte der Gefäßerkrankungstyp nicht ermittelt werden (Tabelle 4). Diese Patienten wurden in die Gruppe „keine Koronarangiographie“ eingeteilt.

Tabelle 4: Gründe für fehlende Angabe des Gefäßerkrankungstyps

\begin{tabular}{|l|c|}
\hline \multicolumn{1}{|c|}{ Grund für fehlende Angabe KHK } & Anzahl Patienten \\
\hline $\begin{array}{l}\text { Sturz mit Reanimationspflichtigkeit: neuroprotektive Kühlung, } \\
\text { Koronarangiographie und PCI erst am Folgetag }\end{array}$ & 1 \\
\hline STEMI übersehen; Behandlung als NSTEMI & 2 \\
\hline Punktion nicht möglich & 1 \\
\hline Typ-A Aortendissektion und sofortige OP & $\mathbf{5}$ \\
\hline Gesamt & 1 \\
\hline
\end{tabular}

Bei 96 Patienten (8 \%) konnte trotz der initialen STEMI-Diagnose koronarangiographisch kein relevant stenosiertes Gefäß ermittelt werden, diese Patienten wurden in die Gruppe Nullgefäßerkrankung (ZVD) eingeteilt.

Der Gruppe Eingefäßerkrankung (SVD) konnten 342 STEMI-Patienten (27 \%) zugeordnet werden, bei diesen Patienten war lediglich das Infarktgefäß relevant stenosiert.

Die zahlenmäßig größte Gruppe bildeten die Patienten mit Mehrgefäßerkrankung (MVD). Insgesamt konnten hier 804 Patienten (64\%) eingegliedert werden. Je nach Vorliegen eines 
$\mathrm{CTO}_{\mathrm{NIRA}}$ wurde diese Gruppe weiter unterteilt. So gab es eine Gruppe von 681 Patienten $(55 \%)$ mit MVD, aber ohne $\mathrm{CTO}_{\mathrm{NIRA}}\left(\mathrm{MVD}-\mathrm{CTO}_{\mathrm{NIRA}}\right)$ und eine Gruppe von 123 Patienten $(10 \%)$ mit MVD und $\mathrm{CTO}_{\text {NIRA }}\left(\mathrm{MVD}+\mathrm{CTO}_{\mathrm{NIRA}}\right)$. Letztere ließ sich zudem weiter in eine Gruppe von 108 Patienten (9 \%) mit MVD und ausschließlich einem $\mathrm{CTO}_{\text {NIRA }}\left(\mathrm{MVD}+1 \mathrm{CTO}_{\mathrm{NIRA}}\right)$ sowie in eine Gruppe von 15 Patienten $(1 \%)$ mit MVD und mehr als einem $\mathrm{CTO}_{\mathrm{NIRA}}\left(\mathrm{MVD}+>1 \mathrm{CTO}_{\mathrm{NIRA}}\right.$ ) unterteilen (Abbildung 2 und 3).

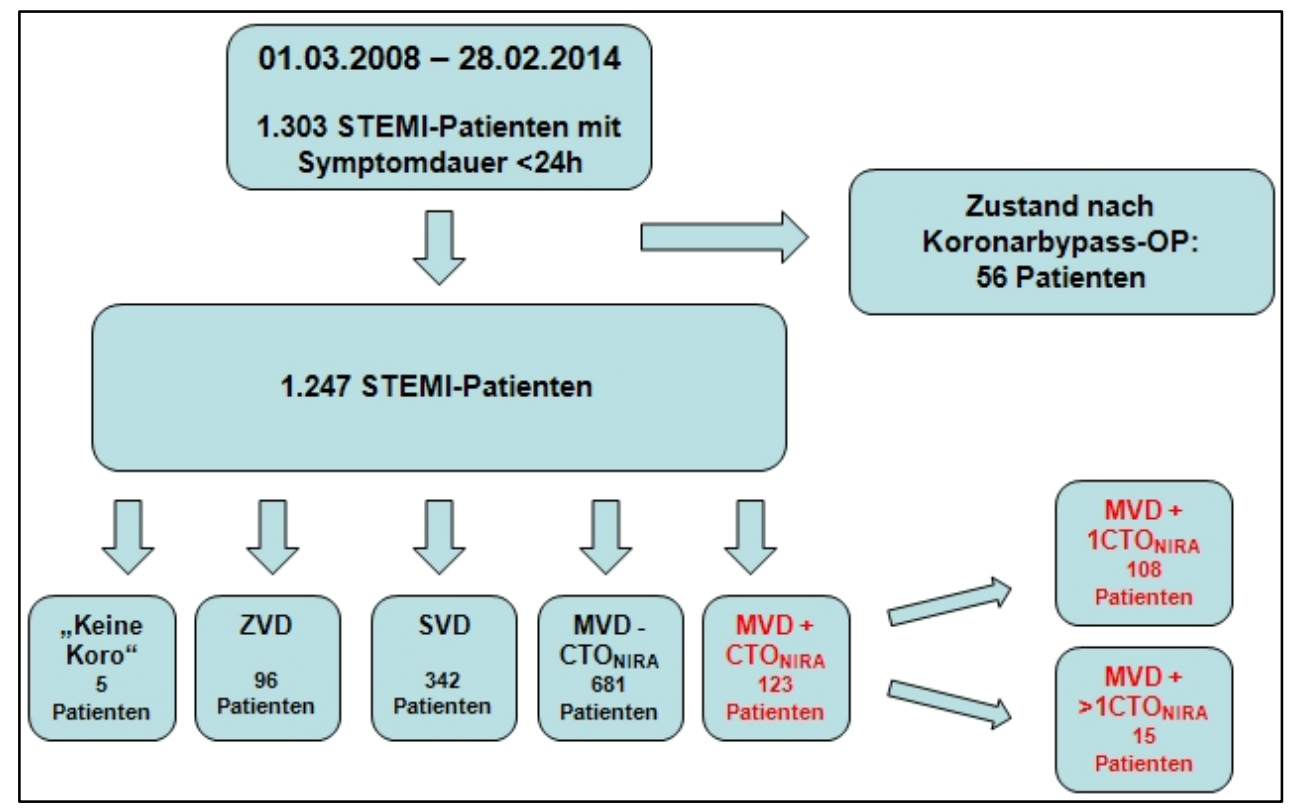

Abbildung 2: Flussdiagramm Patienteneinschluss

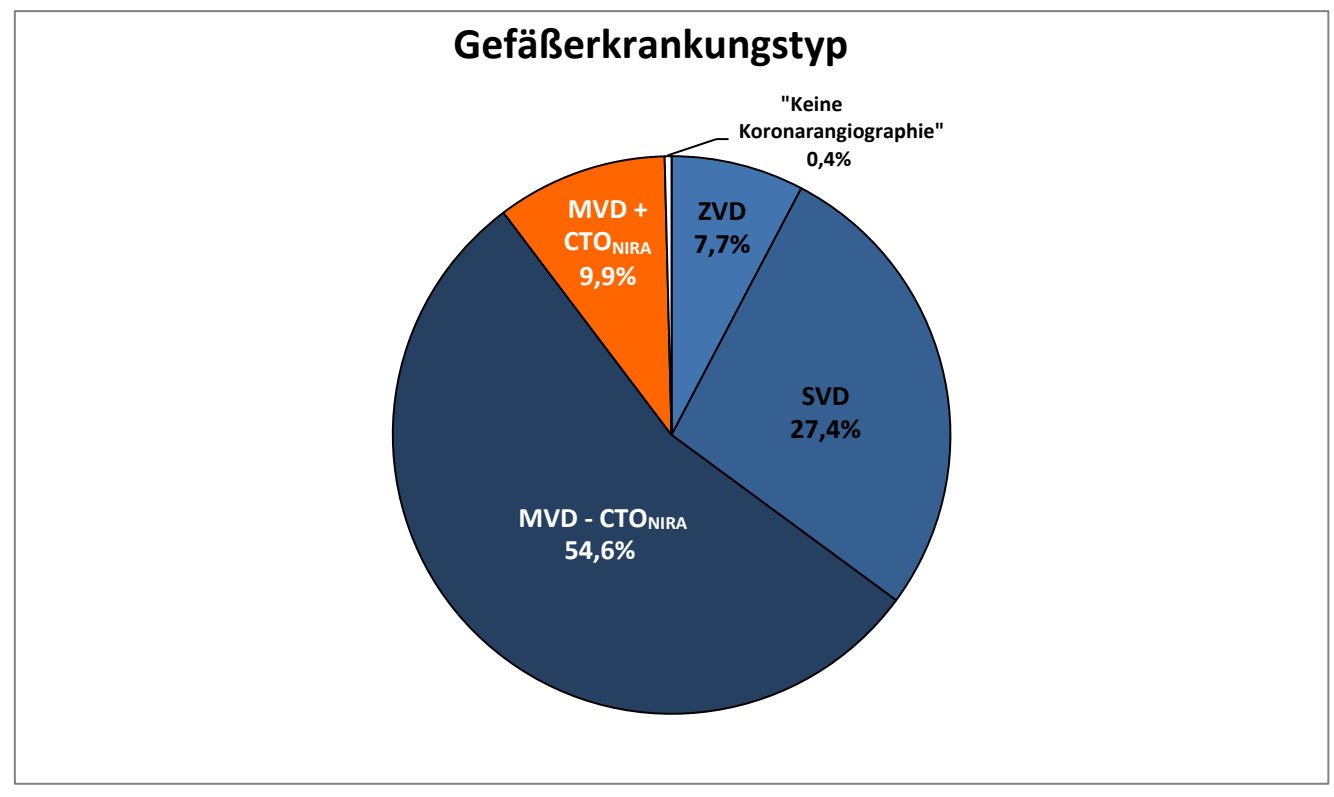

Abbildung 3: Anteil des Gefäßerkrankungstyps der Patienten in \% (Gesamtgruppe; $\mathrm{n}=1.247)$ 


\subsubsection{Transportart und Patientenherkunft}

Insgesamt wurden 726 Patienten (58 \%) während der Rufbereitschaft, also werktags zwischen 16:00 - 7:30 und an Wochenenden und Feiertagen aufgenommen. Bei den restlichen 521 Patienten (42\%) erfolgte die Krankenhausaufnahme während der Regeldienstzeit.

Von den 1.247 STEMI-Patienten der Gesamtgruppe kamen 486 (39 \%) aus dem direkten Einzugsgebiet der Universitätsmedizin Göttingen. Insgesamt 762 Patienten (61 \%) wurden vom Einsatzort direkt per Primärtransport in die UMG eingeliefert. Daneben wurden 429 Patienten (34\%) initial in eine Nicht-PCI-Klinik aufgenommen und dann im Verlauf als Sekundärtransport in die UMG verlegt. Zudem stellten sich 37 Patienten (3\%) direkt in der Notaufnahme der UMG vor (Selbstvorsteller), bei 19 Patienten (2\%) ereignete sich der STEMI im Krankenhaus während eines anderweitigen stationären Aufenthaltes in der UMG (Intrahospital-Infarkt; Abbildung 4).

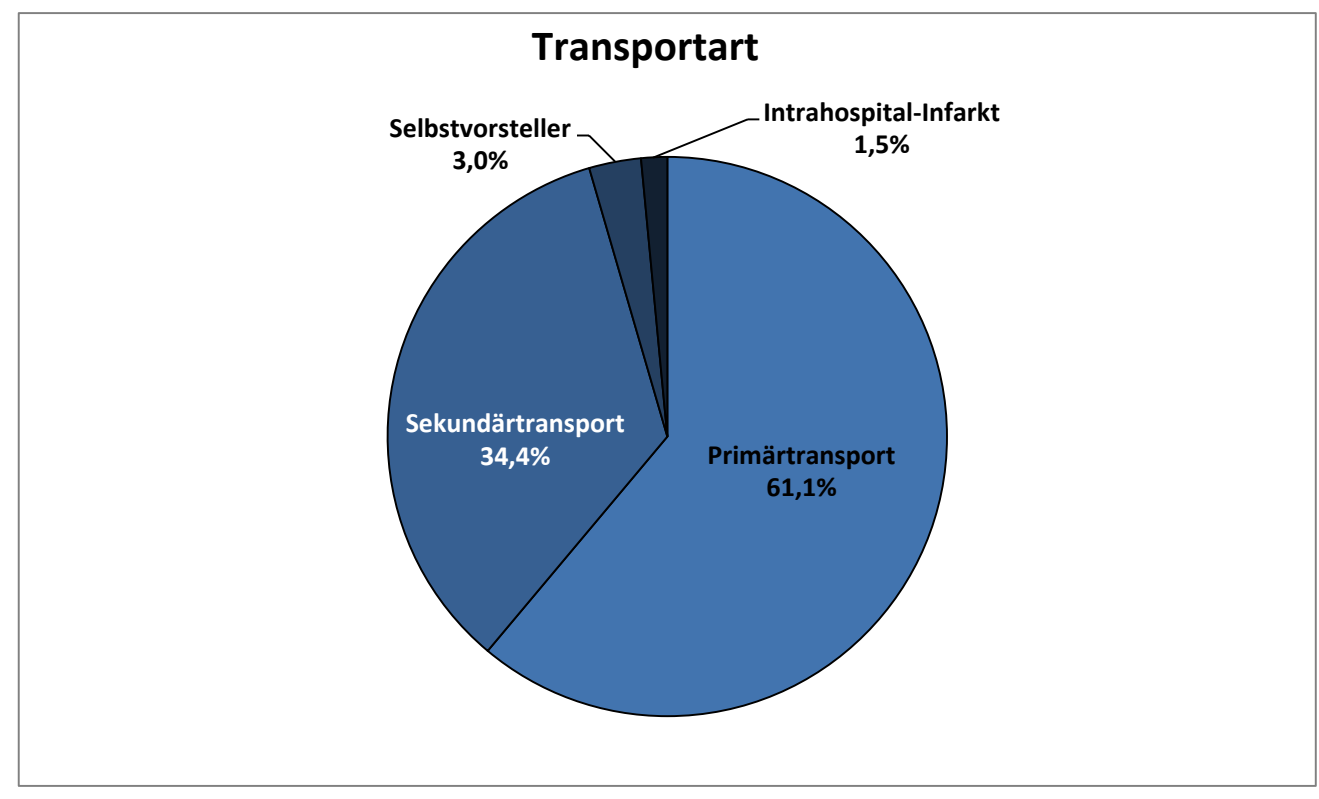

Abbildung 4: Verteilung nach Transportart zur PCI-Klinik in \% (Gesamtgruppe; $\mathrm{n}=1.247)$

Abbildung 5 zeigt die Herkunft aller Patienten mit Primär- und Sekundärtransport ( $\mathrm{n}=1.191)$ auf Basis der Postleitzahl am Einsatzort. Der Radius der Patientenversorgung lag dabei in einzelnen Fällen bei weit über $50 \mathrm{~km}$. 


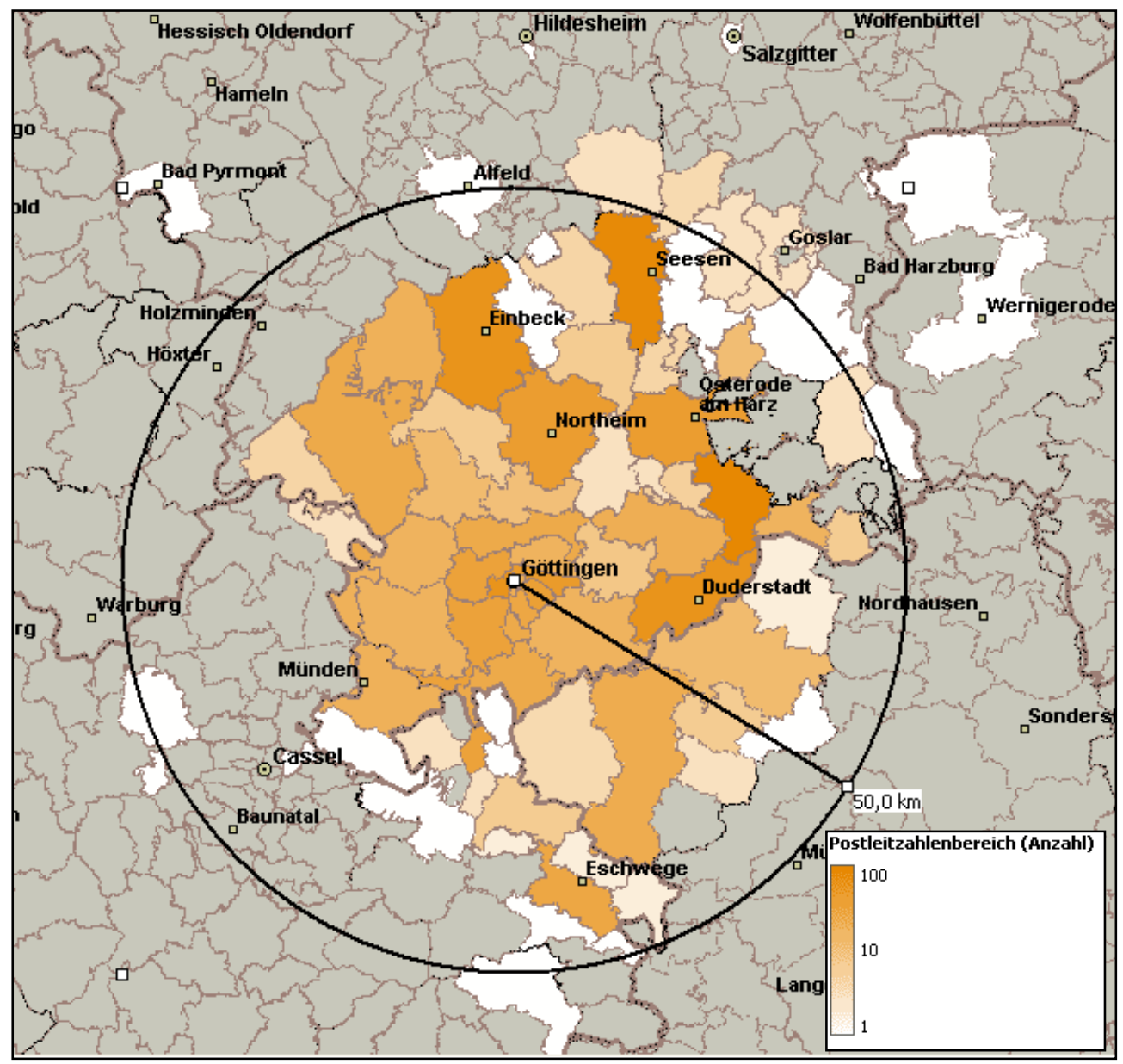

Abbildung 5: Patientenherkunft nach Postleitzahl ( $\mathrm{n}=1.191$ Patienten mit Primär- und Sekundärtransport; Kartenmaterial aus Microsoft $($ MapPoint)

\subsection{Vergleich STEMI-Patienten mit und ohne $\mathrm{CTO}_{\text {NIRA }}$}

Die Basischarakteristika wurden für das Gesamtkollektiv analysiert und nach Koronarstatus jeweils in den relevanten Untergruppen SVD $(\mathrm{n}=342), \mathrm{MVD}_{-} \mathrm{CTO}_{\text {NIRA }}(\mathrm{n}=681)$ und MVD $+\mathrm{CTO}_{\text {NIRA }}(\mathrm{n}=123)$ miteinander verglichen. Zudem wurden potentielle Unterschiede zwischen den Gruppen MVD - $\mathrm{CTO}_{\text {NIRA }}$ und MVD $+\mathrm{CTO}_{\mathrm{NIRA}}$ auf eine statistische Signifikanz hin untersucht.

\subsubsection{Kardiovaskuläre Risikofaktoren}

Von den 1.247 STEMI-Patienten waren 377 (30\%) weiblichen und 870 (70\%) männlichen Geschlechts. Von den 377 Frauen hatten 24 STEMI-Patientinnen (6 \%) einen CTO NIRA $_{\text {. }}$ Bei den Männern war die CTO-Prävalenz höher, hier wiesen 99 von 870 STEMI-Patienten $(11 \%)$ einen $\mathrm{CTO}_{\text {NIRA }}$ auf. Im Vergleich der Gruppen untereinander zeigte sich, dass mit zunehmendem Ausmaß der Gefäßerkrankung der Anteil an männlichen Patienten zunahm. Während in der Gruppe SVD $64 \%$ der Patienten männlich waren, stieg der Anteil männlicher Patienten in der Gruppe MVD - $\mathrm{CTO}_{\mathrm{NIRA}}$ auf $71 \%$ und in der Gruppe MVD 
$+\mathrm{CTO}_{\text {NIRA }}$ auf $80 \%$ (Abbildung 6). Der Unterschied zwischen den Gruppen MVD $\mathrm{CTO}_{\text {NIRA }}$ und MVD $+\mathrm{CTO}_{\text {NIRA }}$ war hierbei statistisch signifikant $(\mathrm{p}=0,036)$.

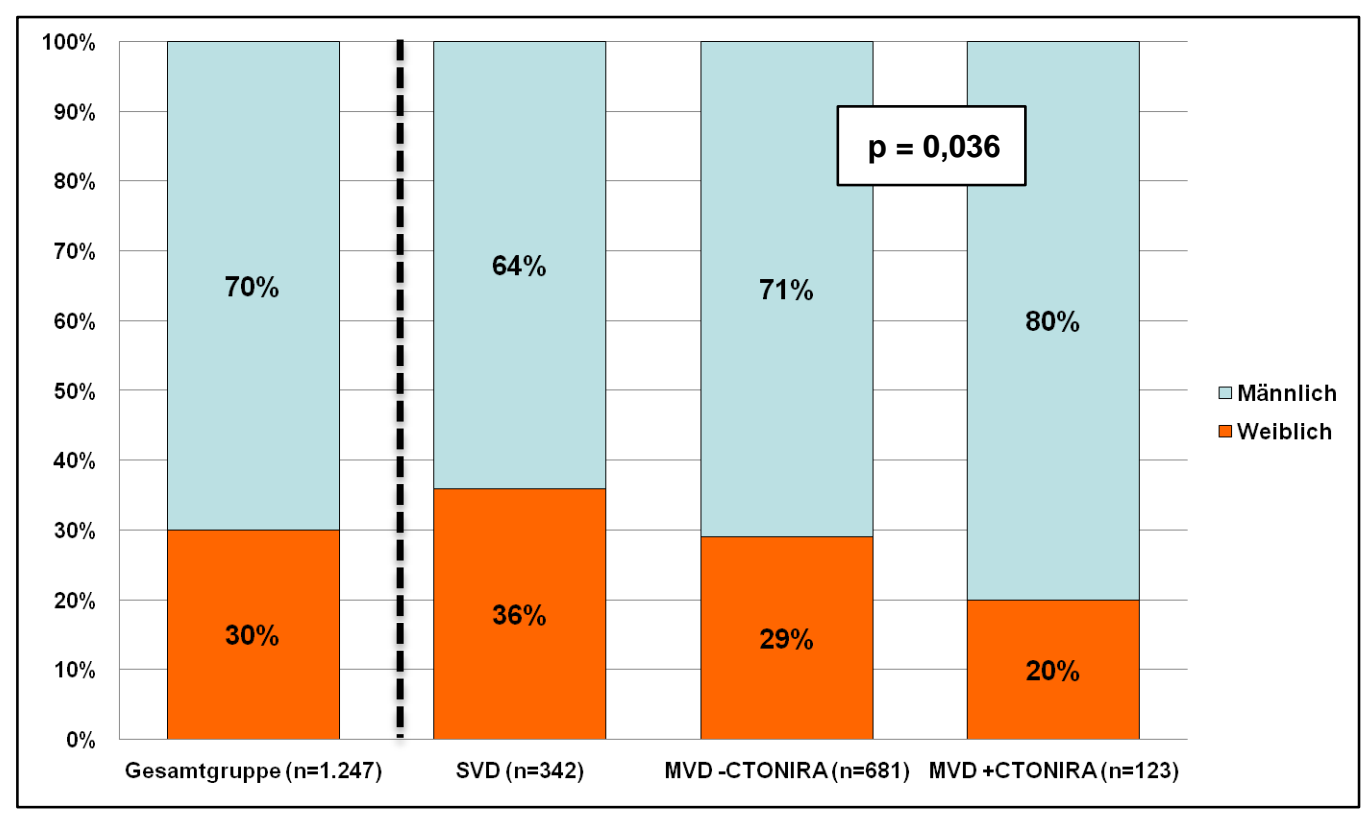

Abbildung 6: Geschlechterverteilung in der Gesamtgruppe und bei Ein- und Mehrgefäß$\mathrm{KHK}$ mit und ohne $\mathrm{CTO}_{\text {NIRA }}$

Das mittlere Alter im Gesamtkollektiv lag bei 64,2 \pm 13,7 Jahren, insgesamt waren 150 Patienten älter als 80 Jahre (12\%). Auch in der Altersverteilung zeigten sich Unterschiede zwischen den einzelnen Gruppen. Je schwerer das Ausmaß der Gefäßerkrankung war, desto höher war auch das Durchschnittsalter der Patienten. Der Altersschnitt lag in der Gruppe MVD -CTO ${ }_{\text {NIRA }}$ bei 66,1 $\pm 12,9$ und in der Gruppe MVD + CTO $_{\text {NIRA }}$ bei $66,3 \pm$ 12,2 Jahren, dieser Unterschied war statistisch nicht signifikant ( $p=0,836$; Abbildung 7).

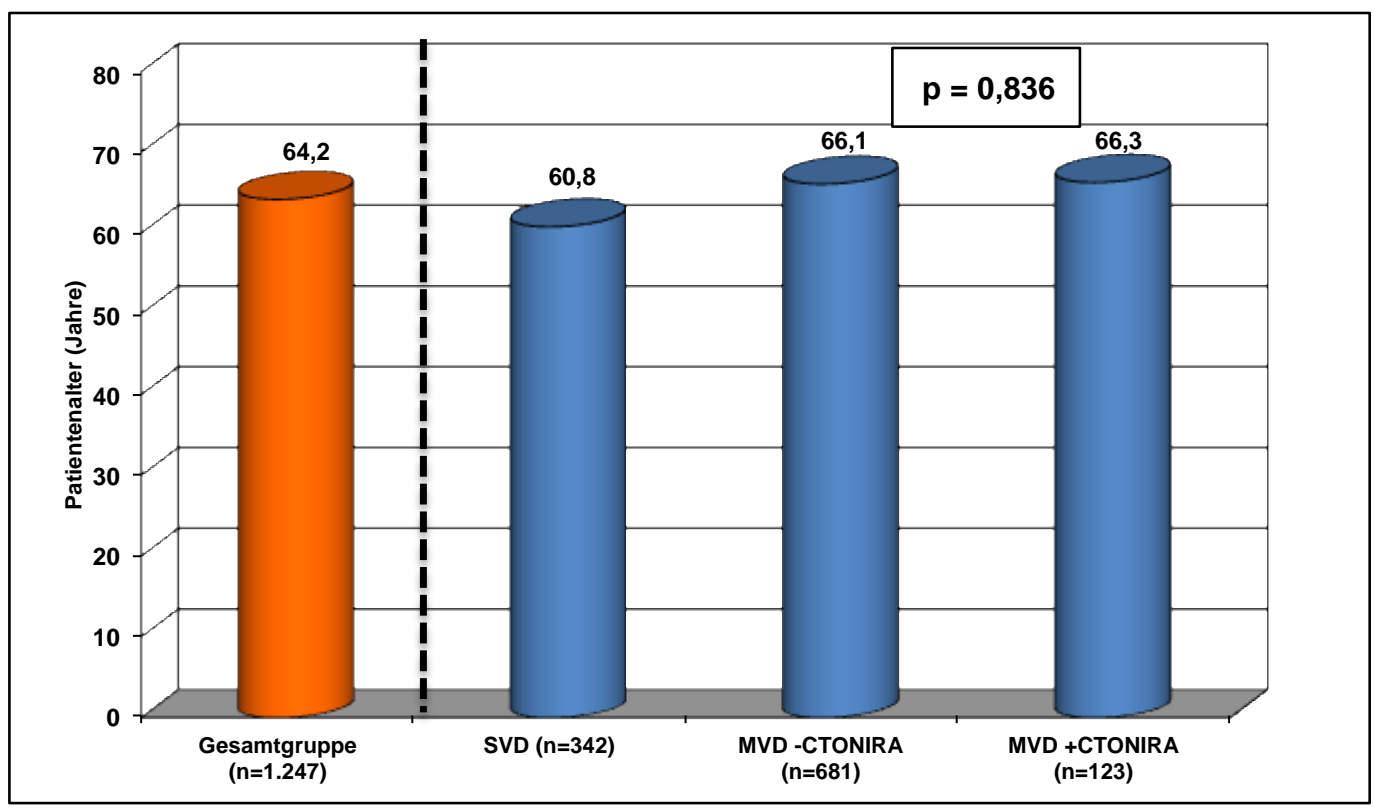

Abbildung 7: Durchschnittliches Patientenalter in der Gesamtgruppe und bei Ein- und Mehrgefäß-KHK mit und ohne CTO $_{\text {NIRA }}$ 
STEMI-Patienten mit $\mathrm{CTO}_{\text {NIRA }}$ waren somit im Durchschnitt fünfeinhalb Jahre älter als STEMI-Patienten mit SVD. Während in der Gruppe SVD 9\% $(\mathrm{n}=32)$ der Patienten älter als 80 Jahre waren, waren dies in der Gruppe MVD - $\mathrm{CTO}_{\text {NIRA }} 12 \%(\mathrm{n}=85)$ und in der Gruppe MVD $+\mathrm{CTO}_{\mathrm{NIRA}} 16 \%\left(\mathrm{n}=20\right.$; p-Wert MVD $-\mathrm{CTO}_{\mathrm{NIRA}}$ vs. MVD $+\mathrm{CTO}_{\mathrm{NIRA}}=$ $0,252)$.

In der Gesamtgruppe betrug der BMI im Durchschnitt 27,3 $\pm 4,42 \mathrm{~kg} / \mathrm{m}^{2}$ und lag somit nach WHO-Definition im Bereich der Präadipositas $\left(25-29,9 \mathrm{~kg} / \mathrm{m}^{2}\right)$. Im Vergleich der Untergruppen MVD - $\mathrm{CTO}_{\mathrm{NIRA}}$ und MVD $+\mathrm{CTO}_{\mathrm{NIRA}}$ zeigten sich bezüglich des BMI nur geringfügige, nicht-signifikante Unterschiede $(p=0,316)$.

Dagegen präsentierten sich mit Blick auf den Anteil weiterer kardiovaskulärer Risikofaktoren in den einzelnen Untergruppen teilweise deutliche Unterschiede.

Die größte Diskrepanz fand sich beim Anteil der Patienten mit Diabetes mellitus. Dieser war in der Gruppe der Patienten mit $\mathrm{CTO}_{\mathrm{NIRA}}$ erheblich höher als in den Vergleichsgruppen (SVD $13 \%$, MVD -CTO ${ }_{\text {NIRA }} 20 \%$, MVD +CTO NIRA $_{3} 3 \%$; Abbildung 8). Zwischen den Gruppen MVD - $\mathrm{CTO}_{\mathrm{NIRA}}$ und MVD $+\mathrm{CTO}_{\mathrm{NIRA}}$ konnte dabei ein hochsignifikanter Unterschied ermittelt werden $(p=0,001)$. In der Gesamtgruppe aller STEMI-Patienten lag der Anteil der Patienten mit DM bei $19 \%(\mathrm{n}=237)$. Von diesen 237 STEMI-Patienten mit DM hatten $17 \%$ einen $\mathrm{CTO}_{\text {NIRA }}(\mathrm{n}=41)$.

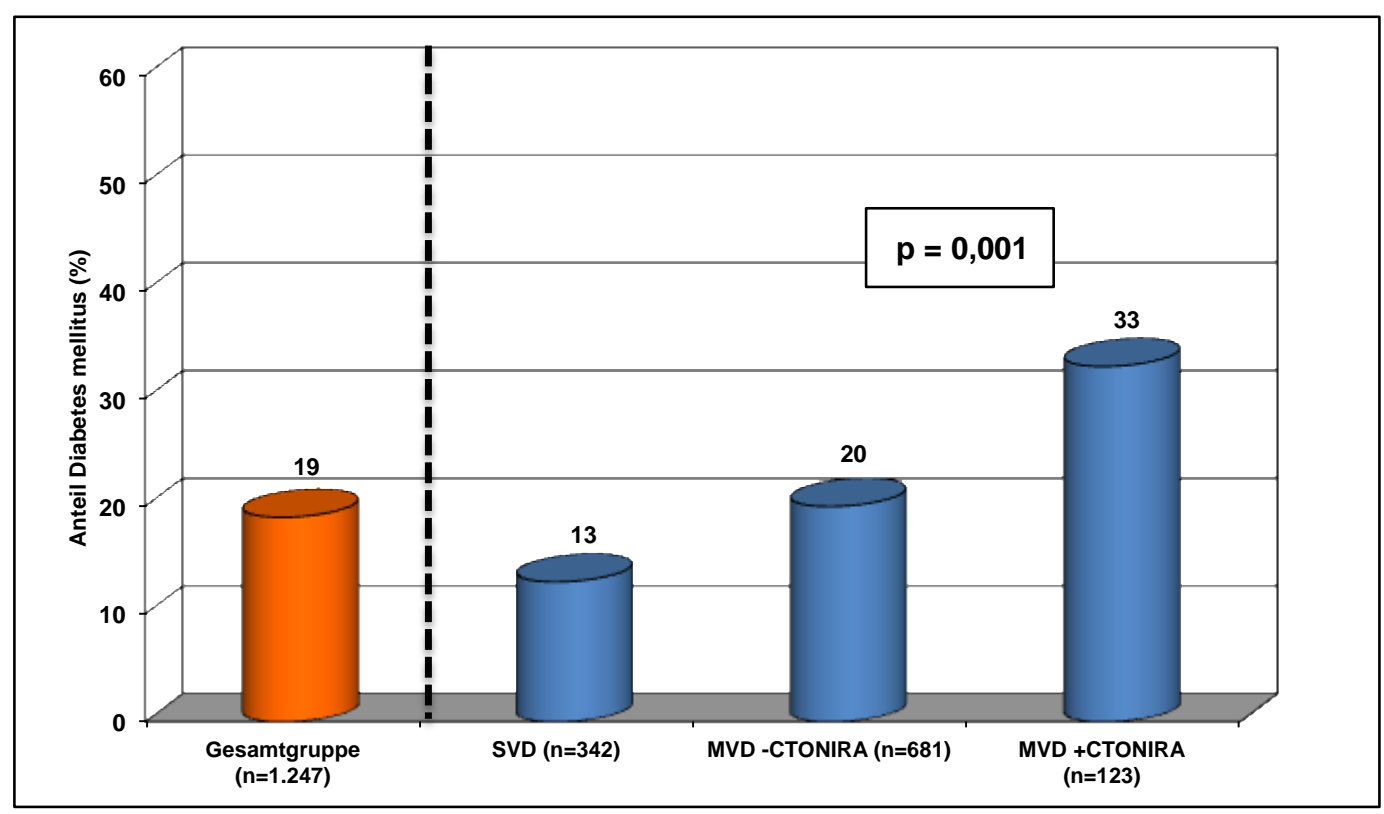

Abbildung 8: Anteil der Patienten mit Diabetes mellitus in der Gesamtgruppe und bei Einund Mehrgefäß-KHK mit und ohne $\mathrm{CTO}_{\mathrm{NIRA}}$

Deutlich milder und zudem nicht signifikant fiel der Unterschied bei den Risikofaktoren Hypertonus (SVD $54 \%$, MVD -CTO ${ }_{\text {NIRA }} 68 \%$, MVD $+\mathrm{CTO}_{\text {NIRA }} 62 \%$ ) und Hyperlipidämie (SVD $29 \%$, MVD -CTO ${ }_{\text {NIRA }} 35 \%$, MVD $+\mathrm{CTO}_{\text {NIRA }} 33 \%$ ) aus, wobei hier sogar 
jeweils die Gruppe MVD ohne CTO $_{\text {NIRA }}$ den größten Patientenanteil aufwies. Beim Anteil der Patienten mit positiver Familienanamnese (SVD $25 \%$, MVD -CTO NIRA $_{2} 22 \%$, MVD $+\mathrm{CTO}_{\text {NIRA }} 18 \%$ ) und Rauchen (SVD $49 \%$, MVD - $\mathrm{CTO}_{\text {NIRA }} 42 \%$, MVD +CTO ${ }_{\text {NIRA }} 39 \%$; Abbildung 9) zeigte sich ein umgekehrtes Bild, der größte Anteil an Patienten mit dem jeweiligen Risikofaktor fand sich hier jeweils in der Gruppe der Patienten mit SVD.

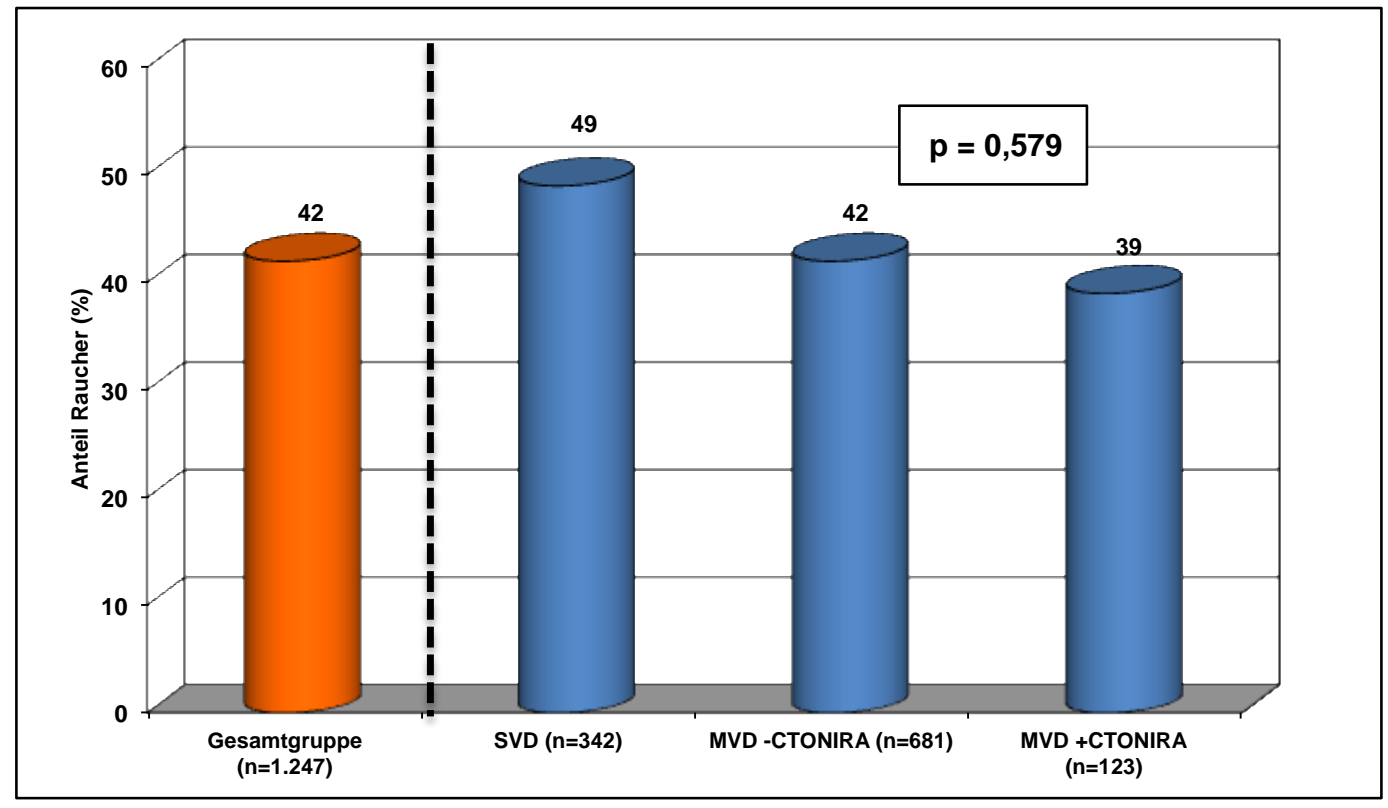

Abbildung 9: Anteil der Raucher in der Gesamtgruppe und bei Ein- und Mehrgefäß-KHK mit und ohne $\mathrm{CTO}_{\mathrm{NIRA}}$

\subsubsection{Vorerkrankungen}

Neben den Risikofaktoren wurden auch die relevanten Vorerkrankungen der Patienten analysiert. Mit steigender Schwere der Gefäßerkrankung wuchs hier, wenn auch in unterschiedlichem Ausmaß und jeweils statistisch nicht signifikant, der Anteil dieser Vorerkrankungen am jeweiligen Kollektiv. Besonders ausgeprägt war dieser Unterschied zwischen den Gruppen beim Anteil an Patienten mit früherem Myokardinfarkt (SVD 7 \%, MVD - $\mathrm{CTO}_{\text {NIRA }} 16 \%$, MVD + CTO $_{\text {NIRA }} 19 \%$; Abbildung 10) sowie Apoplex (SVD $6 \%$, MVD - $\mathrm{CTO}_{\text {NIRA }} 6 \%$, MVD + $\mathrm{CTO}_{\text {NIRA }} 10 \%$; Abbildung 11) in der Vorgeschichte. Auch bezüglich des Anteils an Patienten mit einer Nierenfunktionsstörung zeigt sich ein ähnliches Bild. Während in der Gruppe SVD und MVD - $\mathrm{CTO}_{\text {NIRA }} 9 \%(\mathrm{n}=32) \mathrm{bzw} .8 \%$ $(\mathrm{n}=53)$ der Patienten eine Niereninsuffizienz hatten, waren dies in der Gruppe MVD $+\mathrm{CTO}_{\text {NIRA }} 13 \%(\mathrm{n}=16)$ der Patienten (p-Wert MVD $-\mathrm{CTO}_{\text {NIRA }}$ vs. MVD $\left.+\mathrm{CTO}_{\mathrm{NIRA}}=0,057\right)$.

Bei den Patienten mit einer PCI in der Vorgeschichte gab es dagegen nur einen sehr geringen Unterschied zwischen den Gruppen MVD - $\mathrm{CTO}_{\text {NIRA }}$ und MVD +CTO $\mathrm{NIRA}(14 \%$ vs. $15 \%, \mathrm{p}=0,661)$. 


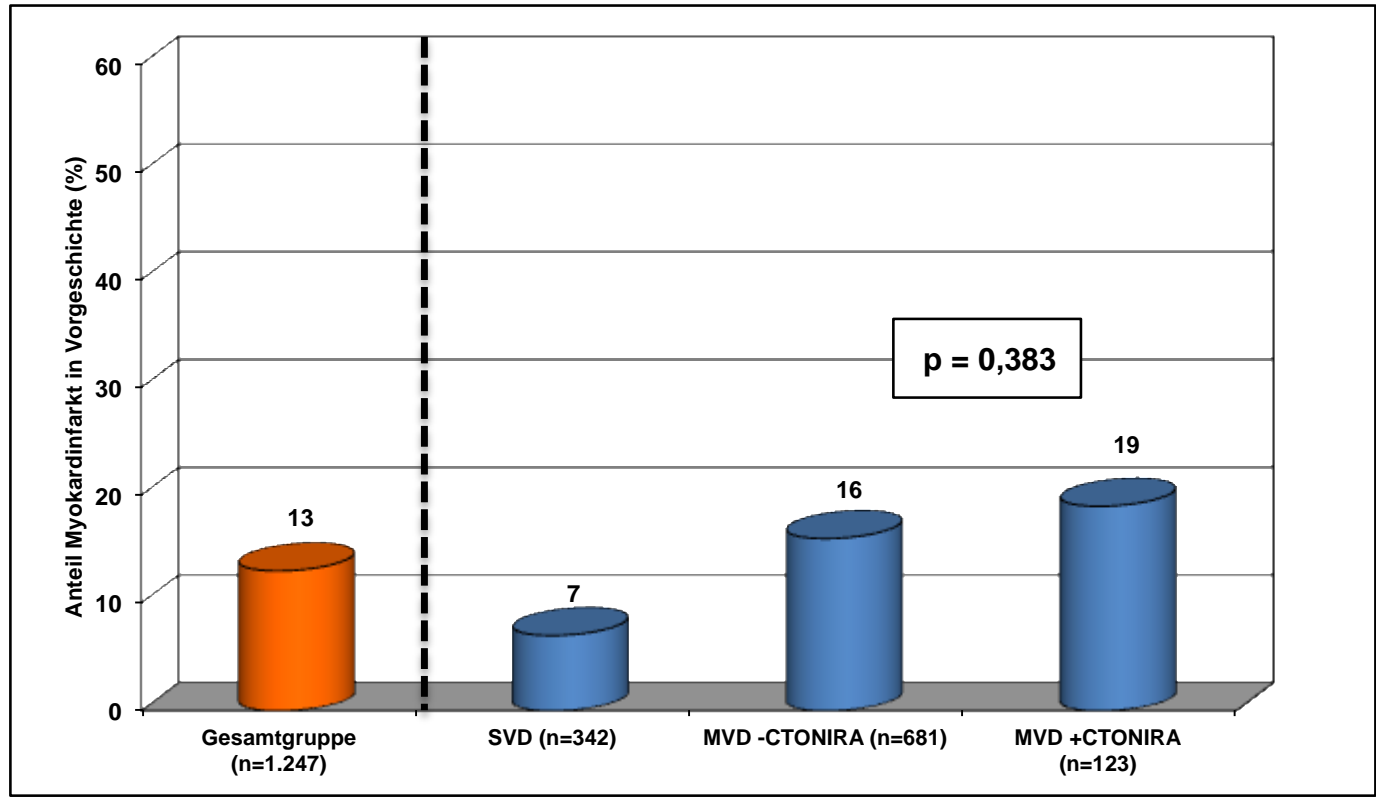

Abbildung 10: Anteil Patienten mit Myokardinfarkt in der Vorgeschichte in der Gesamtgruppe und bei Ein- und Mehrgefäß-KHK mit und ohne CTO $_{\text {NIRA }}$

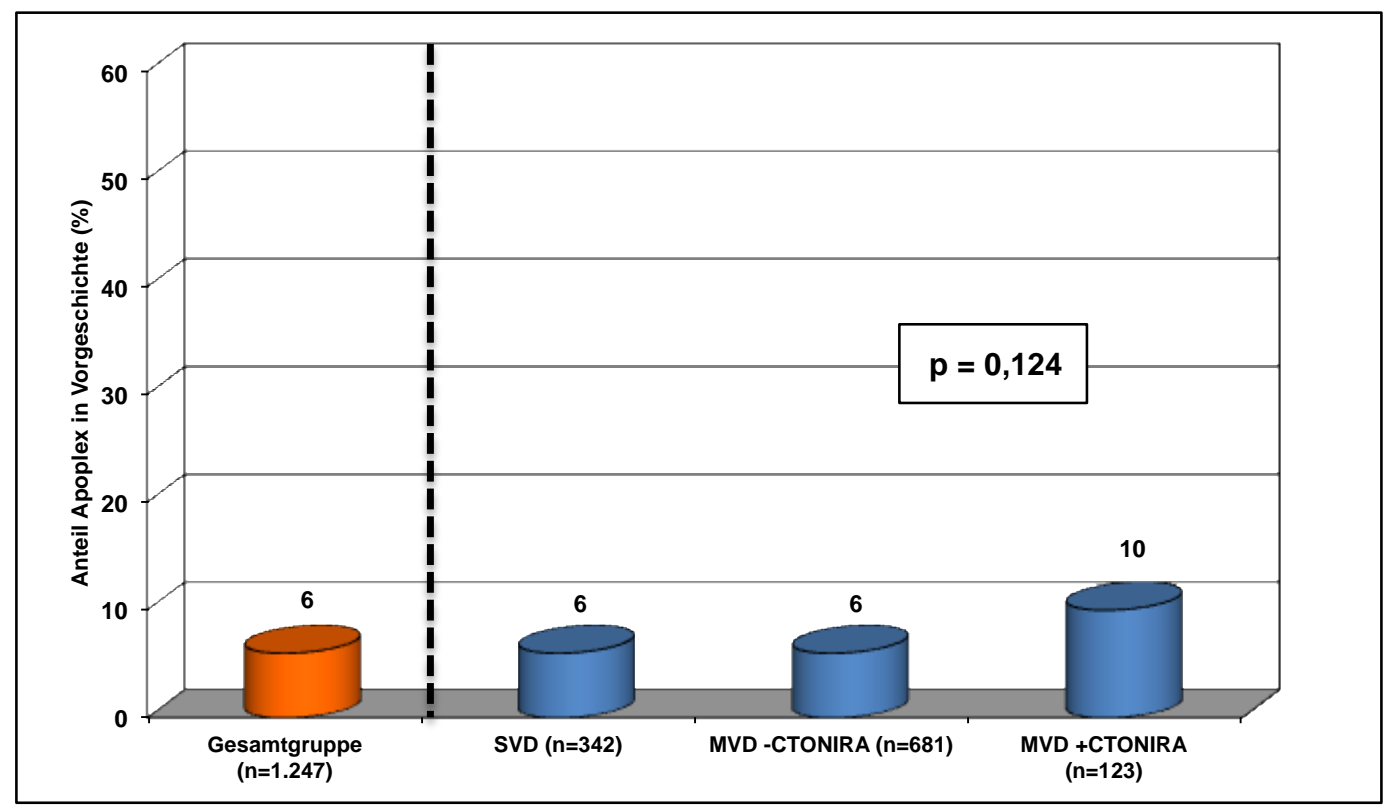

Abbildung 11: Anteil Patienten mit Apoplex in der Vorgeschichte in der Gesamtgruppe und bei Ein- und Mehrgefäß-KHK mit und ohne $\mathrm{CTO}_{\text {NIRA }}$

\subsubsection{Infarktlokalisation}

Mit Hilfe des initialen STEMI-EKG am Einsatzort wurde die Lokalisation des akuten Infarktes bestimmt. In der Gesamtgruppe präsentierten sich $47 \%$ der Patienten mit einem Hinterwandinfarkt, $43 \%$ erlitten einen Vorderwandinfarkt, während $7 \%$ einen Lateralwandinfarkt aufwiesen und bei $3 \%$ ein mutmaßlich neu aufgetretener Linksschenkelblock diagnostiziert wurde (Abbildung 12). Zwischen den Gruppen MVD -CTO ${ }_{\text {NIRA }}$ und MVD $+\mathrm{CTO}_{\text {NIRA }}$ bestanden dabei keine signifikanten Unterschiede $(\mathrm{p}=0,153)$. 


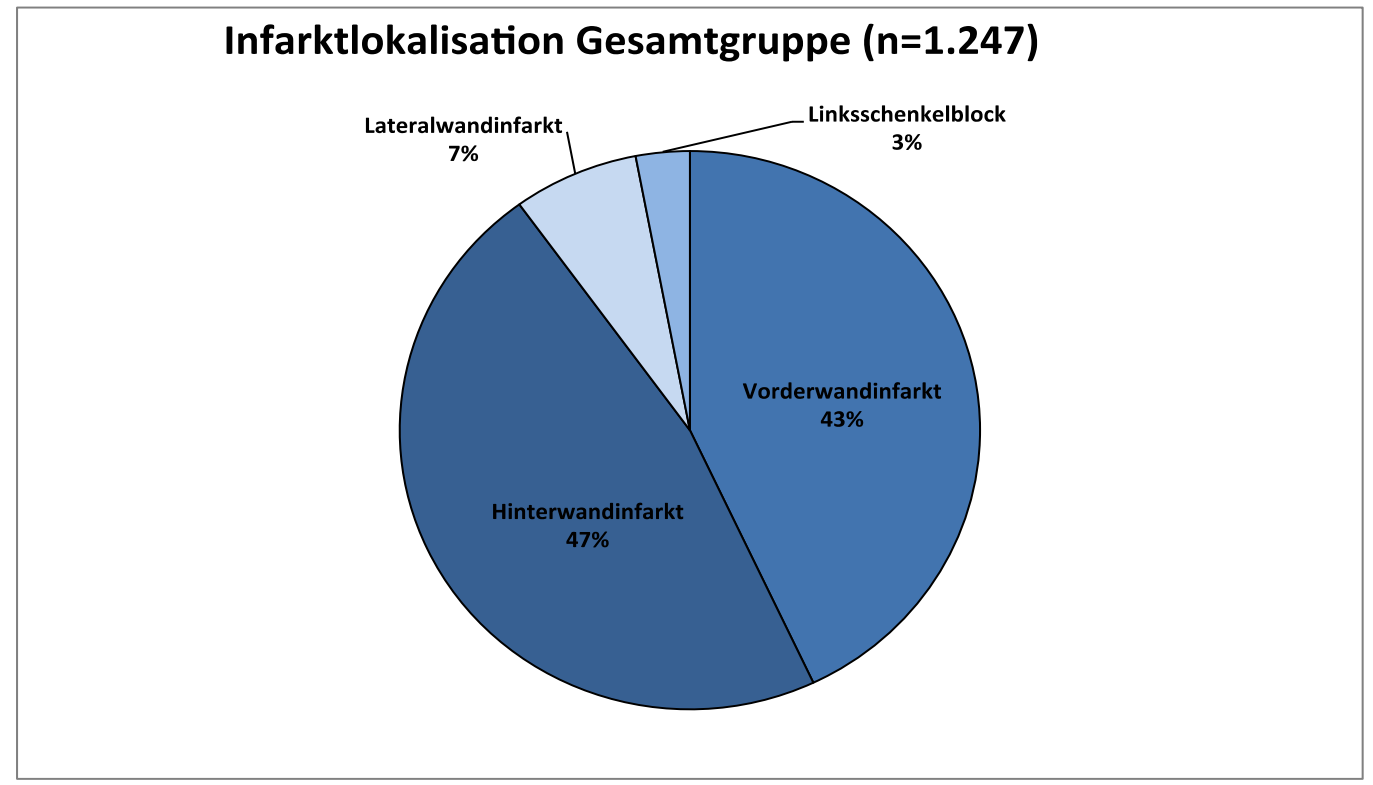

Abbildung 12: Infarktlokalisation im EKG (Gesamtgruppe, $\mathrm{n}=1.247$ )

\subsubsection{LV-Funktion}

Zur Erfassung der Pumpfunktion des linken Ventrikels wurde echokardiographisch die linksventrikuläre Ejektionsfraktion bestimmt. Sie betrug im Mittelwert in der Gesamtgruppe aller STEMI-Patienten 47,6 \%. Im Vergleich der einzelnen Gruppen zeigte sich, dass Patienten mit $\mathrm{CTO}_{\mathrm{NIRA}}$ eine erhebliche Beeinträchtigung der Ejektionsfraktion aufwiesen (EF bei MVD +CTO ${ }_{\text {NIRA }}: 41,7 \%$ ). Dagegen war zwischen den Gruppen SVD und MVD - $\mathrm{CTO}_{\text {NIRA }}$ kein Unterschied zu erkennen (EF bei SVD: 48,3 \%; EF bei MVD $\mathrm{CTO}_{\text {NIRA }}: 48,0 \%$. Die Unterschiede zwischen den Gruppen MVD - CTO $_{\text {NIRA }}$ und $+\mathrm{CTO}_{\text {NIRA }}$ waren hoch signifikant $(\mathrm{p}<0,001)$. Einschränkend ist anzumerken, dass bei einem relevanten Anteil der Patienten (27\%) die LVEF für diese Arbeit nicht bestimmt worden war. Insgesamt fehlten etwas mehr Angaben zur Auswurfsfraktion in der Gruppe MVD $+\mathrm{CTO}_{\text {NIRA }}$, ein Grund hierfür ist u. a. die hohe Krankenhaussterblichkeit in dieser Patientengruppe (siehe Kapitel 4.2.7). Mit Blick auf Abbildung 13 präsentiert sich dennoch eindrucksvoll der Einfluss des $\mathrm{CTO}_{\mathrm{NIRA}}$ auf die LV-Funktion bei STEMI-Patienten. Während zwischen den Gruppen SVD und MVD -CTO ${ }_{\text {NIRA }}$ kein Unterschied im Anteil an den verschiedenen Ejektionsfraktions-Untergruppen erkennbar war, zeigte sich in der Gruppe der Patienten mit MVD $+\mathrm{CTO}_{\mathrm{NIRA}}$ ein signifikant höherer Anteil an Patienten mit schlechter LV-Funktion: $14 \%(\mathrm{n}=17)$ aller STEMI-Patienten mit MVD $+\mathrm{CTO}_{\text {NIRA }}$ hatten eine Ejektionsfraktion $\leq 30 \%$, während in den Gruppen SVD sowie MVD -CTO NIRA lediglich $5 \%(\mathrm{n}=16)$ bzw. $6 \%(\mathrm{n}=38)$ eine Ejektionsfraktion $\leq 30 \%$ aufwiesen (p-Wert

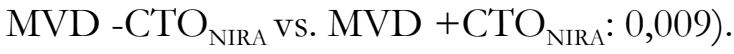




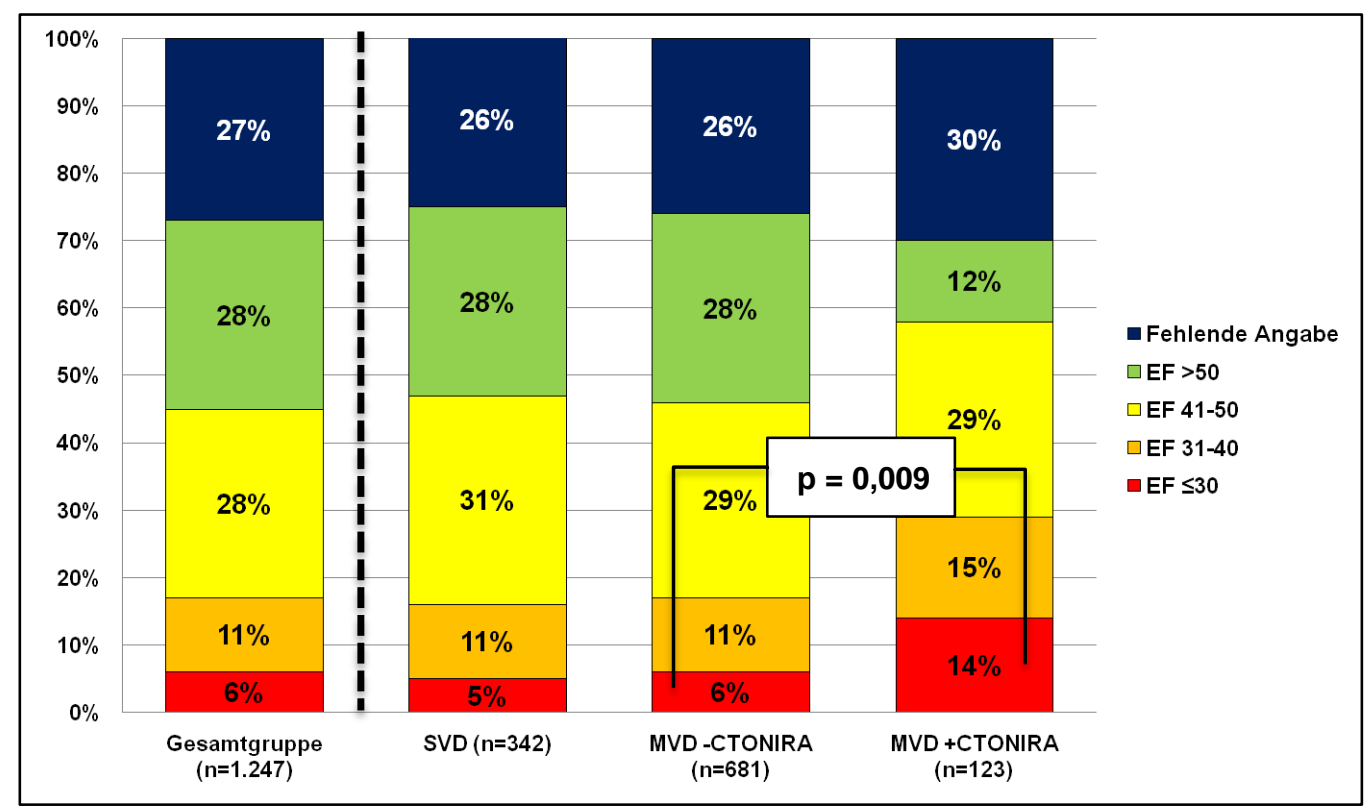

Abbildung 13: Anteil der LV-Ejektionsfraktionsbereiche in der Gesamtgruppe und bei Einund Mehrgefäß-KHK mit und ohne $\mathrm{CTO}_{\text {NIRA }}$

\subsubsection{Risikoprofil und Komplikationen}

Zur präprozeduralen Risikoabschätzung wurde im Rahmen der Patientenaufnahme der TIMI Risk Score bestimmt. Dieser betrug im Mittelwert in der Gesamtgruppe der STEMIPatienten 4,14 $\pm 2,49$. Dabei stieg der Score mit zunehmender Gefäßerkrankung an (SVD 3,75 $\pm 2,38 ;$ MVD -CTO $\left.{ }_{\text {NIRA }} 4,23 \pm 2,49 ; \mathrm{MVD}+\mathrm{CTO}_{\mathrm{NIRA}} 4,91 \pm 2,56\right)$. Es bestand ein signifikanter Unterschied zwischen den Mittelwerten der Gruppen MVD -CTO NIRA und $+\mathrm{CTO}_{\text {NIRA }}(\mathrm{p}=0,009)$. Über die Hälfte der Patienten mit MVD $+\mathrm{CTO}_{\text {NIRA }}(54 \%, \mathrm{n}=66)$ wies einen TIMI Risk Score $\geq 5$ auf (SVD: $33 \%$; MVD -CTO NIRA $_{4} 42 \%$; Abbildung 14).

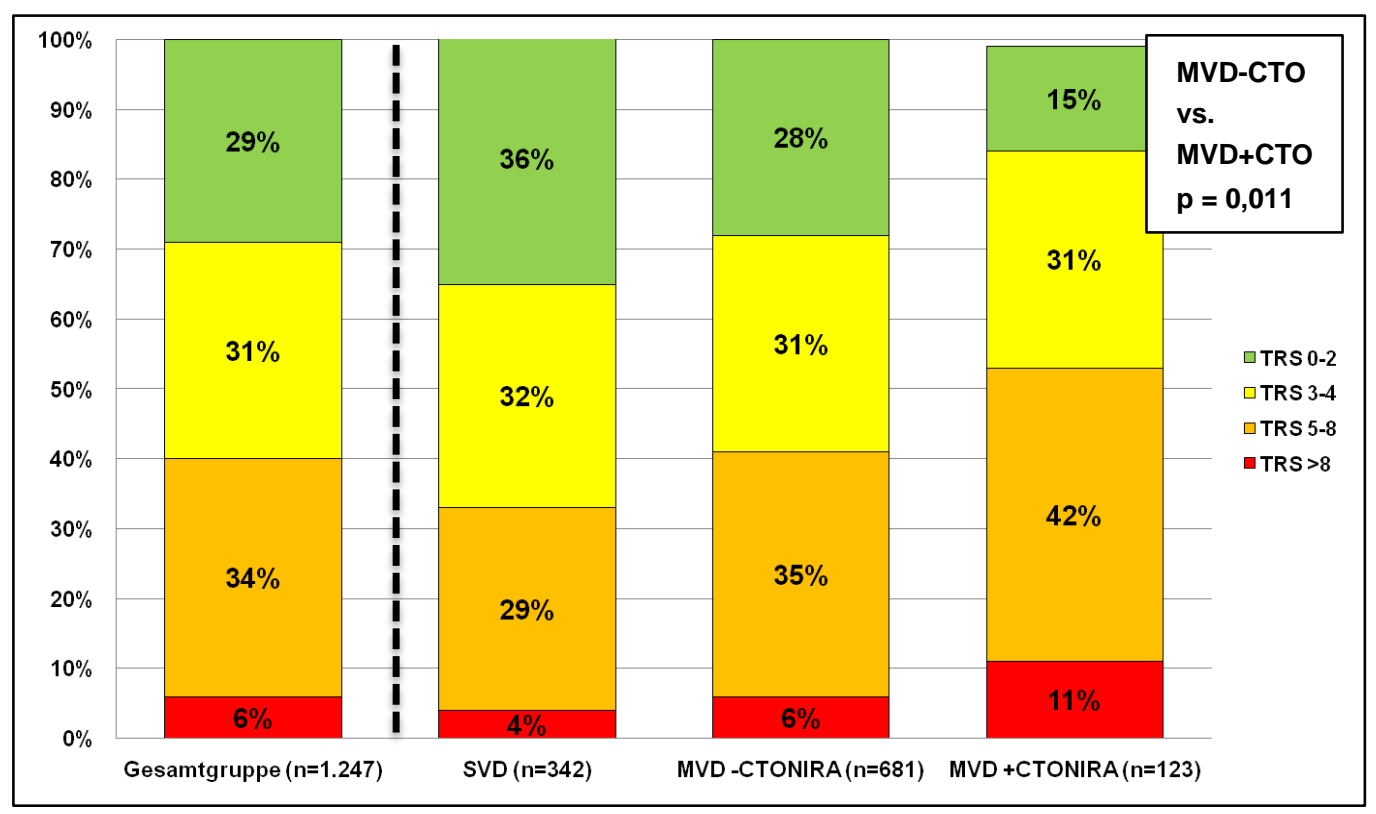

Abbildung 14: Anteil TIMI Risk Score-Gruppen in der Gesamtgruppe und bei Ein- und Mehrgefäß-KHK mit und ohne $\mathrm{CTO}_{\text {NIRA }}$ 
Zur weiteren Risikostratifikation wurden die Patienten anhand der Killip-Klassifikation in unterschiedliche Risikogruppen eingeteilt. Abbildung 15 stellt den Anteil der einzelnen Killip-Klassen in den verschiedenen Gruppen dar. Auch hier zeigt sich die immense Bedeutung eines chronischen Verschlusses. Zwischen den Patientengruppen SVD und MVD - $\mathrm{CTO}_{\text {NIRA }}$ war kein Unterschied in den Killip-Klassen-Anteilen zu erkennen, annähernd drei von vier Patienten erreichten das Krankenhaus ohne die Zeichen einer Herzinsuffizienz (Killip I) und lediglich $14 \%$ (SVD) bzw. $15 \%\left(\right.$ MVD -CTO $\left.{ }_{\text {NIRA }}\right)$ der Patienten wurden im kardiogenen Schock eingeliefert. Dagegen zeigte sich in der Gruppe der STEMI-Patienten mit MVD + CTO $_{\text {NIRA }}$ ein eindeutig anderes Bild: Nur $50 \%$ der Patienten mit MVD $+\mathrm{CTO}_{\text {NIRA }}$ wurden ohne Herzinsuffizienzzeichen aufgenommen und jeder dritte Patient aus dieser Gruppe erreichte die UMG im kardiogenen Schock. Die Unterschiede beim Anteil an Patienten im kardiogenen Schock zwischen den Gruppen MVD - $\mathrm{CTO}_{\mathrm{NIRA}}$ und $+\mathrm{CTO}_{\mathrm{NIRA}}$ waren hierbei hoch signifikant $(15 \%$ vs. $33 \%$; p < 0,001). Bei weiterer Analyse in der Gruppe MVD $+\mathrm{CTO}_{\text {NIRA }}$ wurde zudem ersichtlich, dass Patienten mit $>1 \mathrm{CTO}_{\text {NIRA }}$ mit $47 \%$ ein besonders hohes Risiko haben, im Rahmen eines STEMI einen kardiogenen Schock zu erleiden.

Insgesamt wurden von den 1.247 Patienten der Gesamtgruppe 198 Patienten (16 \%) im kardiogenen Schock ins Krankenhaus eingeliefert. Von diesen 198 STEMI-Patienten mit kardiogenem Schock hatten 41 Patienten $(21 \%)$ eine MVD $+\mathrm{CTO}_{\text {NIRA }}$.

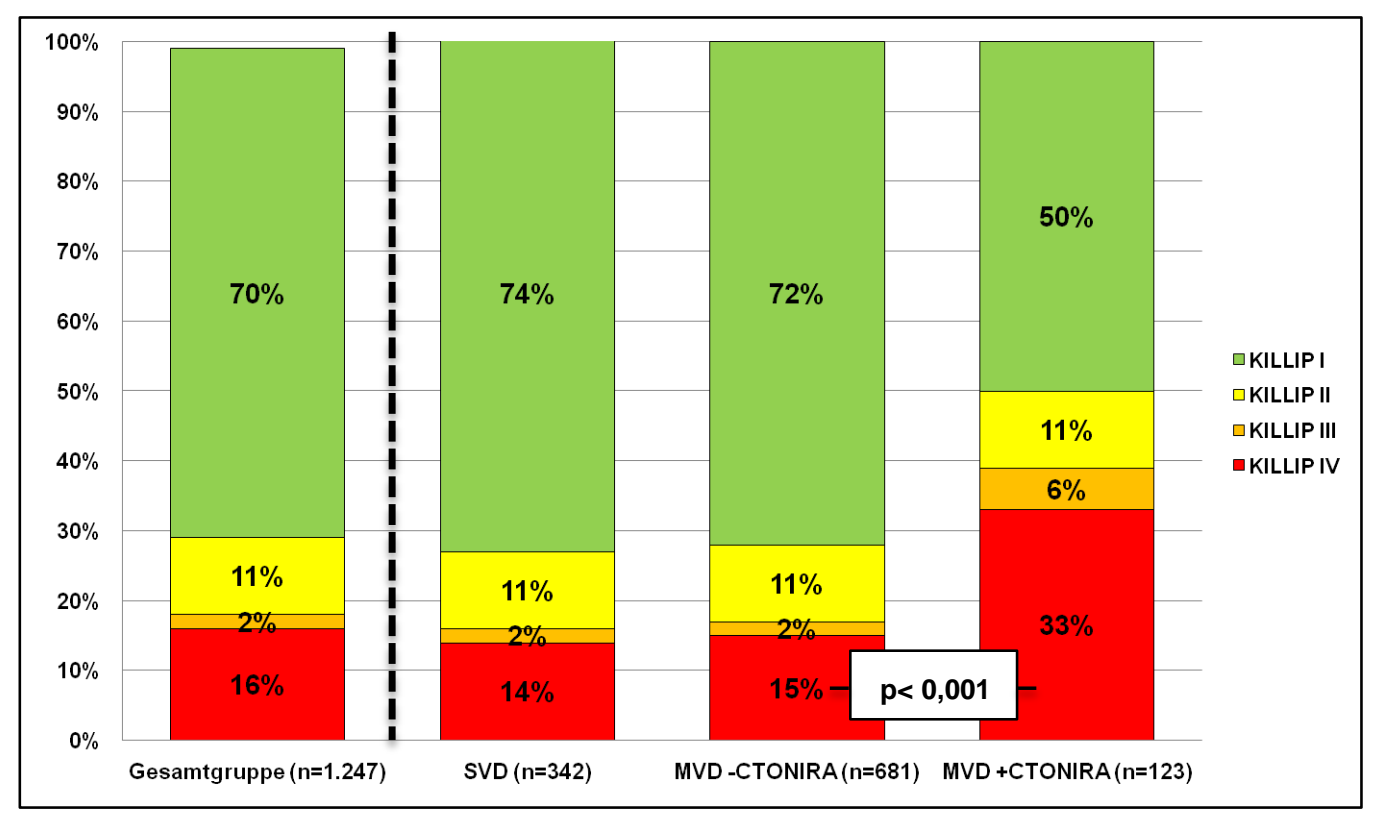

Abbildung 15: Anteil Killip-Klassifikation in der Gesamtgruppe und bei Ein- und Mehrgefäß-KHK mit und ohne CTO $_{\text {NIRA }}$

Ein ähnliches Bild zeigte sich auch beim Anteil der Patienten mit präklinischer Reanimation. In der Gesamtgruppe wurden 134 Patienten präklinisch mechanisch reanimiert, dies waren $11 \%$ aller Patienten mit STEMI. Zwischen den Gruppen SVD (11\%) und 
MVD - $\mathrm{CTO}_{\mathrm{NIRA}}(10 \%)$ war kein Unterschied auszumachen. Dagegen stellte sich in der Gruppe MVD $+\mathrm{CTO}_{\text {NIRA }}$ ein sprunghafter Anstieg des Anteils der Patienten mit präklinischer Reanimation auf $17 \%(\mathrm{n}=21)$ dar (Abbildung 16). Auch hier zeigte sich ein signifikanter Unterschied zwischen den Gruppen MVD - $\mathrm{CTO}_{\text {NIRA }}$ und MVD +CTO ${ }_{\text {NIRA }}$ $(\mathrm{p}=0,021)$. Von den Patienten mit MVD $+>1 \mathrm{CTO}_{\mathrm{NIRA}}$ wurden $27 \%$ der Patienten präklinisch reanimiert.

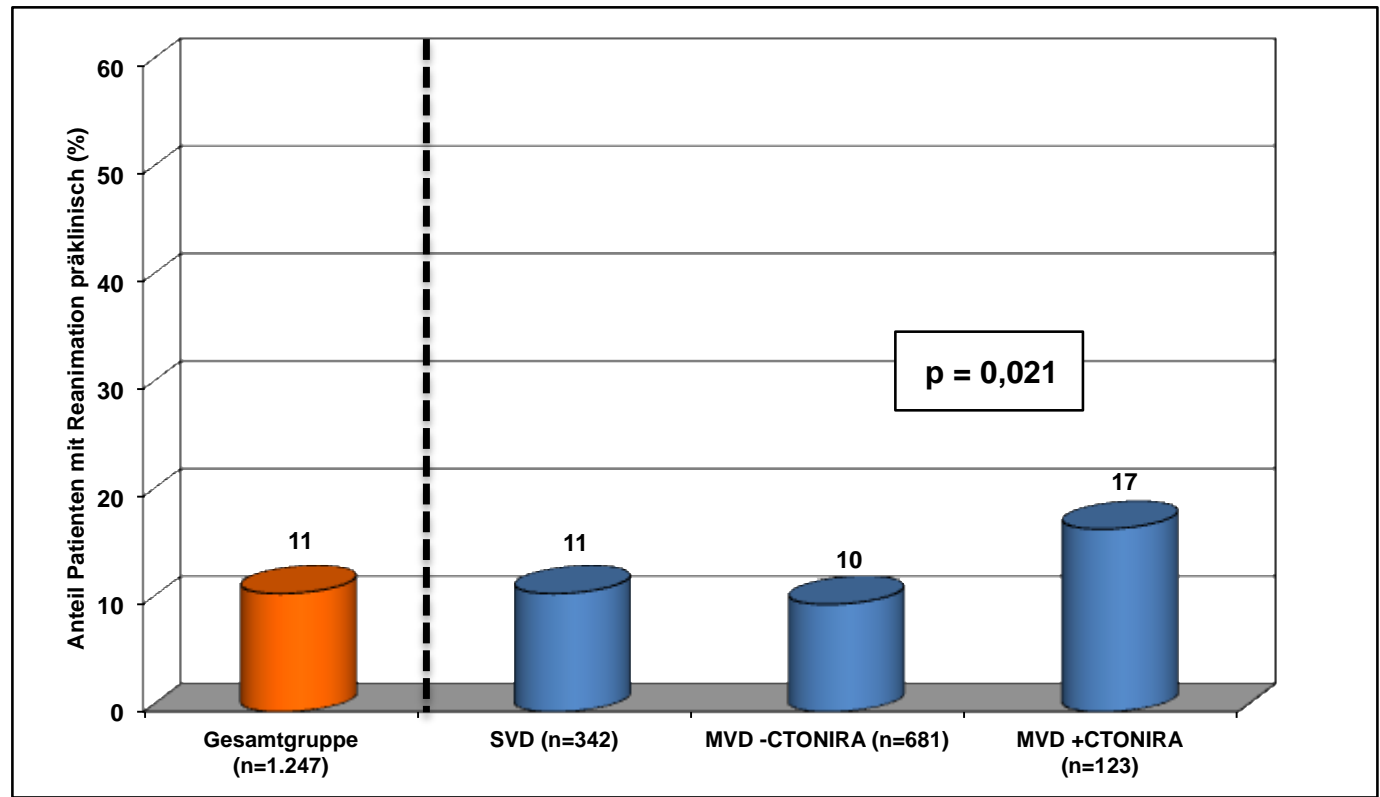

Abbildung 16: Anteil der Patienten mit präklinischer mechanischer Reanimation in der Gesamtgruppe und bei Ein- und Mehrgefäß-KHK mit und ohne CTO $_{\text {NIRA }}$

\subsubsection{Angiographische und prozedurale Charakteristika}

Im Rahmen der Koronarangiographie wurde in der Gruppe MVD -CTO $\mathrm{NIRA}_{\text {bei }} 50 \%$ der Patienten eine 3-Gefäß-Erkrankung diagnostiziert, in der Gruppe mit MVD +CTO ${ }_{\text {NIRA }}$ wiesen dagegen mit $80 \%$ signifikant mehr Patienten eine 3-Gefäß-KHK auf ( $p<0,001)$.

Von den 1.247 STEMI-Patienten der Gesamtgruppe wurde bei 990 Patienten (79\%) eine primäre PCI durchgeführt. Bei 257 STEMI-Patienten (21 \%) wurde aus den verschiedensten Gründen auf eine primäre PCI verzichtet, 39 von diesen Patienten mussten sich aufgrund des Ausmaßes der Gefäßerkrankung einer Akut-Bypass-OP unterziehen (Tabelle 5). Der Anteil der Patienten, bei denen keine primäre PCI durchgeführt wurde, war im Vergleich der einzelnen Gruppen nicht konstant. Bei Patienten mit MVD $+\mathrm{CTO}_{\mathrm{NIRA}}$ erfolgte signifikant seltener eine primäre PCI als in den Vergleichsgruppen: In der Gruppe der Patienten mit SVD wurde bei 299 Patienten (87 \%) eine primäre PCI durchgeführt, in der Gruppe MVD -CTO ${ }_{\mathrm{NIRA}}$ erfolgte bei 596 Patienten (88\%) eine PCI, während in der Gruppe MVD +CTO ${ }_{\text {NIRA }}$ lediglich 95 Patienten $(77 \%)$ eine primäre PCI

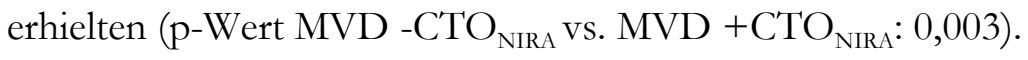


Tabelle 5: Gründe für nicht erfolgte primäre PCI

\begin{tabular}{|l|c|}
\hline \multicolumn{1}{|c|}{ Grund für nicht erfolgte primäre PCI } & Anzahl Patienten \\
\hline Myokarditis & 33 \\
\hline Tako-Tsubo-Kardiomyopathie & 16 \\
\hline Akut-Bypass-OP & 39 \\
\hline Kein Zielgefäß & 76 \\
\hline Drahtpassage technisch nicht möglich & 10 \\
\hline Zusätzlich Hauptstammstenose & 8 \\
\hline Vasospasmus & 3 \\
\hline „Andere Gründe“ & 67 \\
\hline Keine Akut-Koro (s. Kapitel 4.1.1) & 5 \\
\hline Gesamt & 257 \\
\hline
\end{tabular}

Im Rahmen der bei 990 STEMI-Patienten durchgeführten Koronarintervention wurde bei 438 Patienten (44\%) der RIVA, bei 410 Patienten (41\%) die RCA, bei 138 Patienten $(14 \%)$ der RCX und bei vier Patienten $(0,4 \%)$ der linke Hauptstamm rekanalisiert (Abbildung 17). Beim Vergleich der Gruppen MVD - $\mathrm{CTO}_{\mathrm{NIRA}}$ und $+\mathrm{CTO}_{\mathrm{NIRA}}$ fiel ein signifikanter Unterschied in den Anteilen des jeweiligen rekanalisierten Gefäßes auf $(\mathrm{p}=0,028)$. Während in der Gruppe MVD $+\mathrm{CTO}_{\mathrm{NIRA}}$ bei $38 \%$ der Patienten die RCA und bei $22 \%$ der Patienten der RCX rekanalisiert wurde, waren dies in der Gruppe MVD $\mathrm{CTO}_{\text {NIRA }} 45 \%$ (RCA) bzw. $14 \%$ (RCX).

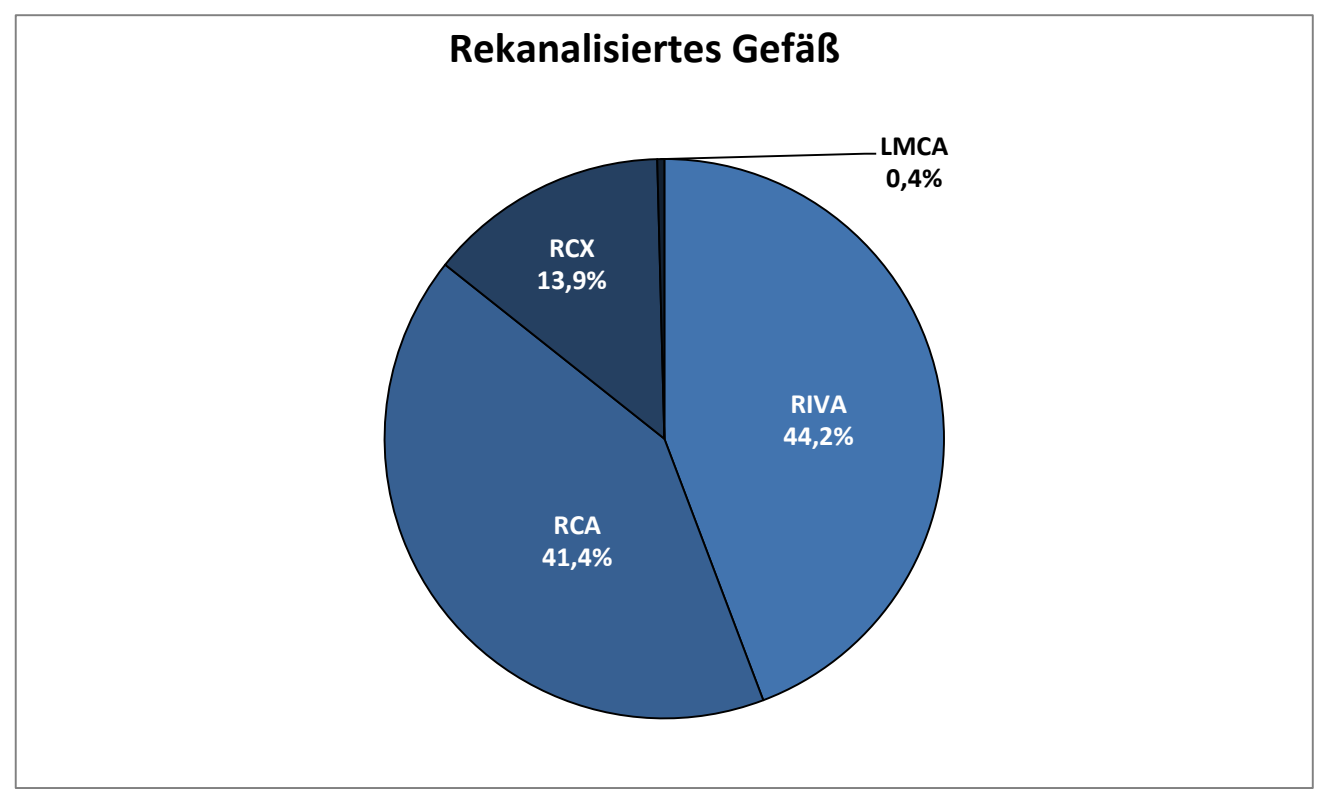

Abbildung 17: Rekanalisiertes Gefäß (Gesamtgruppe, $\mathrm{n}=990)$ 
Um den PCI-Behandlungserfolg zu erfassen, wurde der TIMI Grade Flow in die Gruppen „TIMI Grade Flow 0-II“ (verzögerte bzw. unvollständige/keine Perfusion über die Verschlussstelle hinaus) und „TIMI Grade Flow III“ (Vollständige und umgehende Perfusion) eingeteilt und jeweils vor und nach der primären PCI im Zielgefäß gemessen.

Vor der primären PCI lag der TIMI Grade Flow in allen drei Gruppen in annähernd allen Fällen im Bereich 0-II, wobei auch hier ein nicht signifikanter Unterschied in der Gruppe MVD $+\mathrm{CTO}_{\mathrm{NIRA}}$ ersichtlich war (SVD $96 \%$; MVD -CTO ${ }_{\mathrm{NIRA}} 96 \%$; MVD +CTO NIRA $99 \%$; p-Wert MVD -CTO ${ }_{\text {NIRA }}$ vs. MVD +CTO NIRA $_{\text {: }}$ 0,203; Abbildung 18).

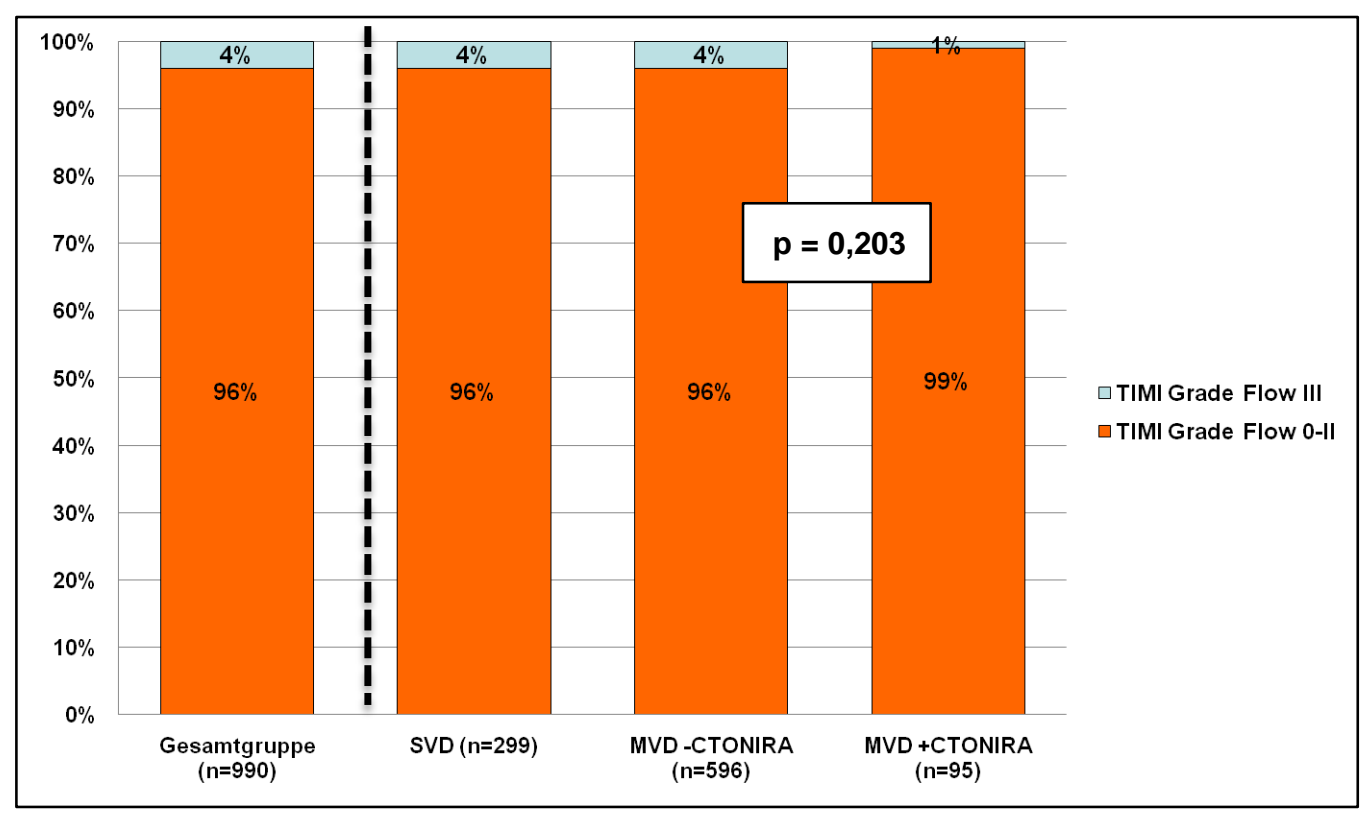

Abbildung 18: Anteil TIMI Grade Flow Vor-PCI in \% in der Gesamtgruppe und bei Einund Mehrgefäß-KHK mit und ohne $\mathrm{CTO}_{\text {NIRA }}$ (Alle Patienten mit STEMI und PCI)

Mit Blick auf den TIMI Grade Flow Nach-PCI ist dabei ein weitaus stärkerer Unterschied zwischen den einzelnen Patientengruppen ersichtlich. Während ein Behandlungserfolg im Sinne eines TIMI-III-Flusses nach PCI im Zielgefäß bei $94 \%$ aller Patienten mit MVD $\mathrm{CTO}_{\text {NIRA }}$ erreicht wurde, konnte eine vollständige Eröffnung des vormals verschlossenen Zielgefäßes in der Gruppe MVD $+\mathrm{CTO}_{\text {NIRA }}$ lediglich bei $88 \%$ der Patienten erreicht werden (Abbildung 19). Der Unterschied im TIMI Grade Flow Nach-PCI zwischen den Gruppen MVD - $\mathrm{CTO}_{\text {NIRA }}$ und MVD $+\mathrm{CTO}_{\text {NIRA }}$ war hierbei statistisch signifikant $(94 \%$ vs. $88 \%$; p-Wert: 0,038). 


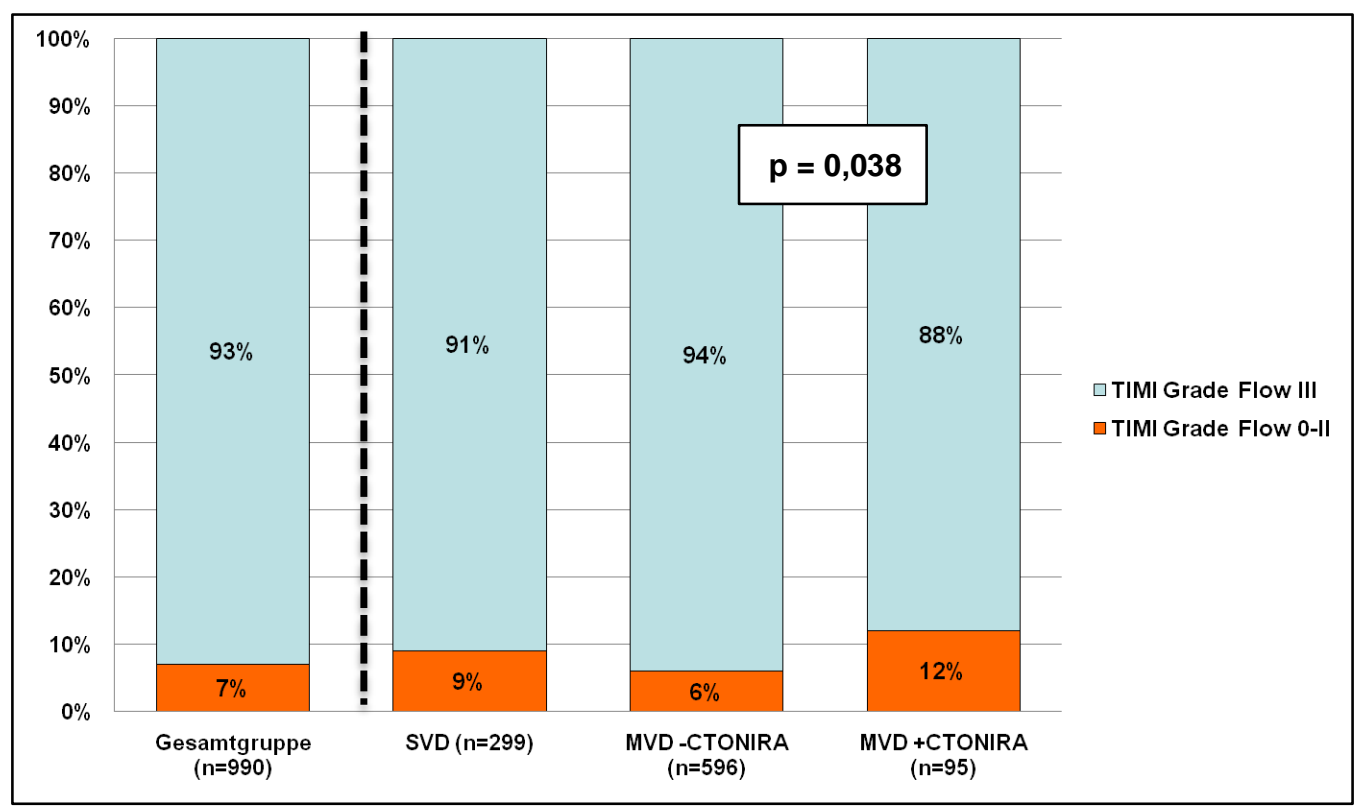

Abbildung 19: Anteil TIMI Grade Flow Nach-PCI in \% in der Gesamtgruppe und bei Einund Mehrgefäß-KHK mit und ohne CTO $_{\text {NIRA }}$ (Alle Patienten mit STEMI und PCI)

Die contact-to-balloon-Zeit betrug in der Gesamtgruppe aller Patienten mit primärer PCI (ausgenommen Patienten mit IIH) im Median 111 Minuten. Mit 114 Minuten war die C2BZeit in der Gruppe MVD+CTO ${ }_{\text {NIRA }}$ am längsten, allerdings war hierbei kein signifikanter Unterschied zwischen den Gruppen MVD - $\mathrm{CTO}_{\mathrm{NIRA}}$ und MVD $+\mathrm{CTO}_{\mathrm{NIRA}}$ festzustellen $(\mathrm{p}=0,53$; Abbildung 20).

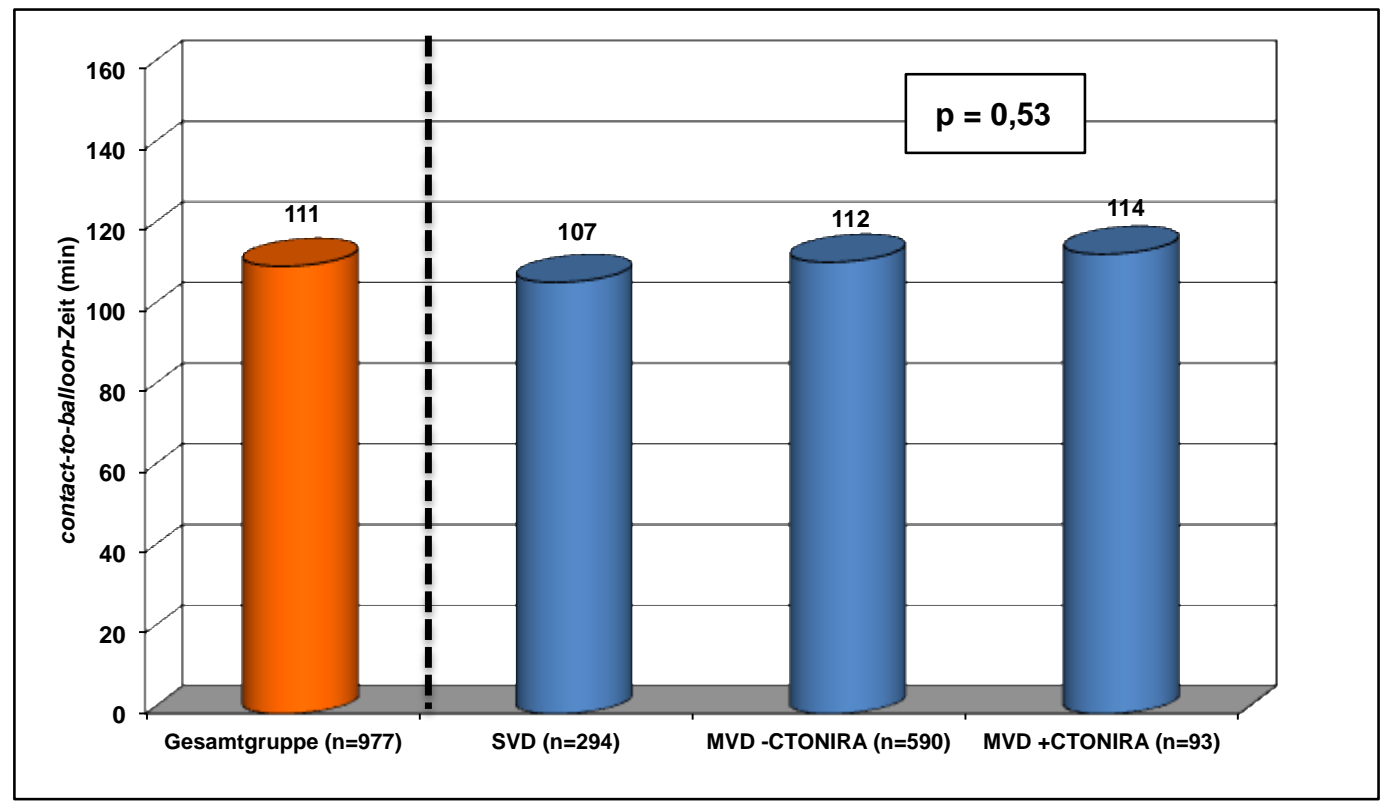

Abbildung 20: C2B-Zeiten im Median in der Gesamtgruppe und bei Ein- und MehrgefäßKHK mit und ohne CTO $_{\text {NIRA }}$ (Alle Patienten mit STEMI und PCI, ohne Patienten mit $\mathrm{IIH})$ 


\subsubsection{Kurzzeitsterblichkeit}

In der Gesamtgruppe aller STEMI-Patienten verstarben während des Krankenhausaufenthaltes 123 Patienten (9,9\%) an den Folgen des Myokardinfarktes. Von allen STEMIPatienten ohne $\mathrm{CTO}_{\text {NIRA }}$ verstarben 8,0 \% (90 von 1.124 Patienten) im Krankenhaus. Im Vergleich der einzelnen Sub-Gruppen nahm mit steigender Komplexität der Gefäßerkrankung die Krankenhaussterblichkeit deutlich zu (SVD 4,7\%; MVD - $\mathrm{CTO}_{\mathrm{NIRA}}$ 9,8 \%; MVD $+\mathrm{CTO}_{\text {NIRA }} 26,8 \%$, Abbildung 21). Der Unterschied in der Krankenhaussterblichkeit zwischen den Gruppen MVD - $\mathrm{CTO}_{\text {NIRA }}$ und MVD $+\mathrm{CTO}_{\text {NIRA }}$ war hierbei hoch signifikant $(\mathrm{p}<0,001)$. Während in der Gruppe der STEMI-Patienten mit MVD +1CTO NIRA $_{25,9 \%}$ der Patienten verstarben, hatten die STEMI-Patienten mit MVD +>1CTO ${ }_{\text {NIRA }}$ mit 33,3\% die höchste Krankenhaussterblichkeit aller Gruppen.

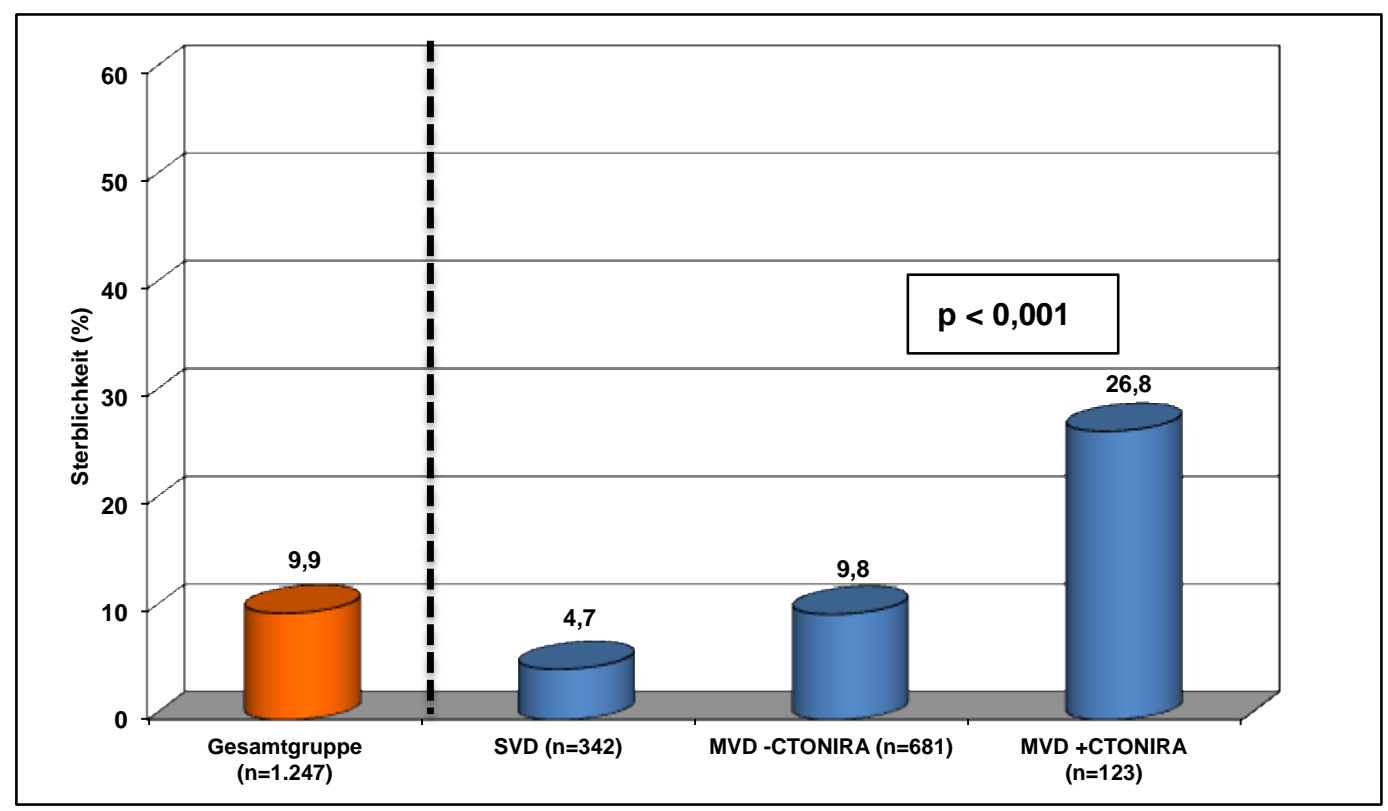

Abbildung 21: Krankenhaussterblichkeit in der Gesamtgruppe und bei Ein- und Mehrgefäß-KHK mit und ohne $\mathrm{CTO}_{\text {NIRA }}$

Mit Blick auf die Sterblichkeit innerhalb von 30 Tagen präsentierte sich ebenfalls ein hoch signifikanter Unterschied $(\mathrm{p}<0,001)$ zwischen den Gruppen MVD -CTO NIRA (Sterblichkeit ${ }_{30 \mathrm{~d}}: 10,7 \%$ ) und MVD $+\mathrm{CTO}_{\text {NIRA }}\left(\right.$ Sterblichkeit $_{30 \mathrm{~d}}: 27,6 \%$ ). Allerdings ist hier zu beachten, dass in der Gesamtgruppe bei insgesamt 115 Patienten (9,2 \%) die Sterblichkeitsangaben aufgrund eines fehlenden Follow-ups nicht erfasst werden konnten.

In Abbildung 22 wird zudem die Krankenhaussterblichkeit bei STEMI-Patienten mit Schock in Abhängigkeit vom Koronargefäßstatus präsentiert. Es zeigte sich, dass unter den STEMI-Patienten im kardiogenen Schock die signifikant höchste Sterblichkeit mit $61 \%$ in

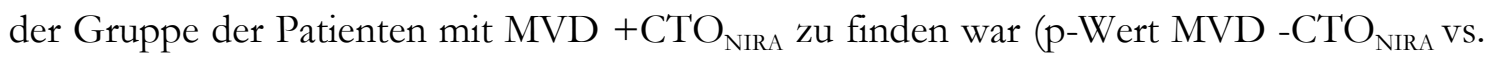
MVD +CTO NIRA $: 0,035)$. 


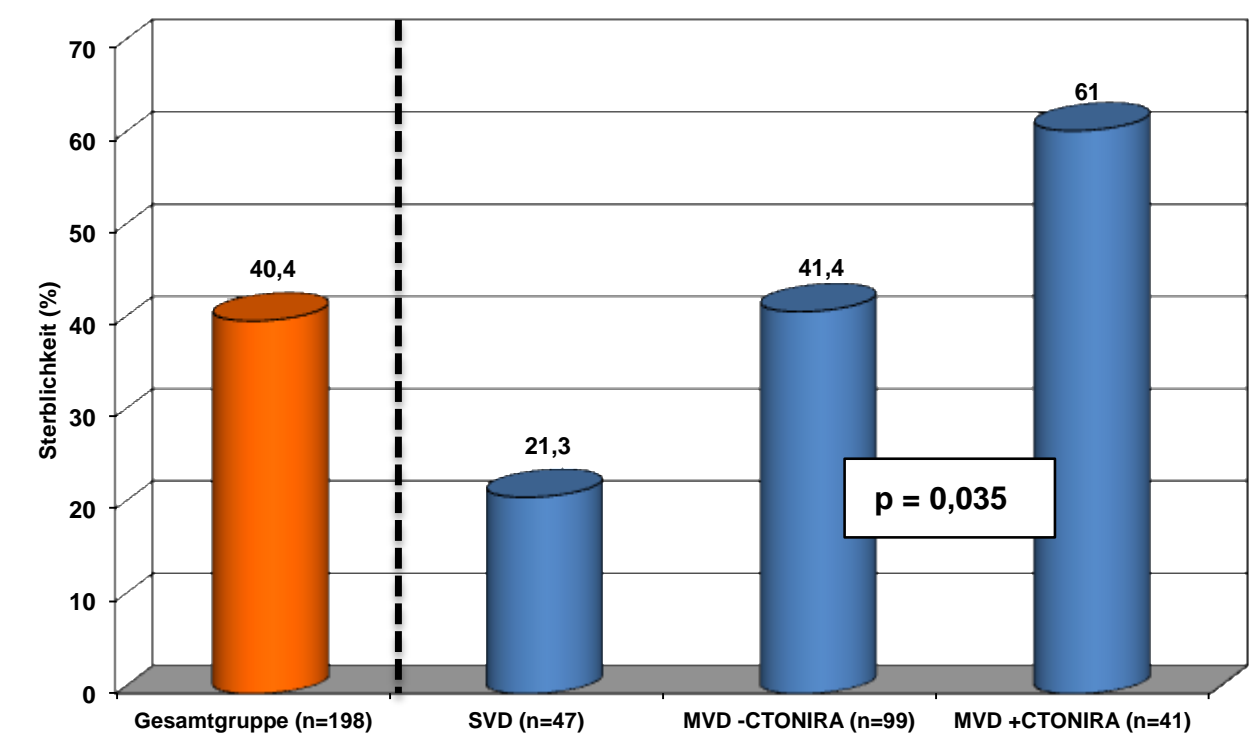

Abbildung 22: Krankenhaussterblichkeit bei Patienten mit Schock in der Gesamtgruppe und bei Ein- und Mehrgefäß-KHK mit und ohne $\mathrm{CTO}_{\text {NIRA }}$

\subsection{STEMI-Patienten mit CTO $_{\text {NIRA }}$}

Wie in Abschnitt 4.1 beschrieben, konnte bei 123 von 1.247 (9,9\%) erfassten STEMIPatienten ein zusätzlich chronischer Verschluss in einem Nicht-Infarkt-Gefäß diagnostiziert werden. Während die Basischarakteristika dieser Patienten mit $\mathrm{CTO}_{\mathrm{NIRA}}$ im vorherigen Abschnitt präsentiert wurden, fokussiert der folgende Ergebnisteil vor allem auf die angiographische Lokalisation des $\mathrm{CTO}_{\text {NIRA }}$, die zugrundeliegenden Versorgungstypen, die vorhandene Kollateralversorgung, sowie Hinweise auf einen bereits früher abgelaufenen Infarkt. Abschließend wird der Einfluss all dieser Faktoren auf die Patientenprognose in Form der Kurzzeitsterblichkeit präsentiert. Unter den 123 Patienten mit CTO $_{\text {NIRA }}$ befanden sich auch 15 Patienten mit multiplen $\mathrm{CTO}_{\text {NIRA }}$ in unterschiedlichen Gefäßen. Da diese Patienten bei der Zuordnung des CTO-Gefäßes doppelt gezählt würden und somit eine Verfälschung der Aussagen die Folge wäre, findet im folgenden Abschnitt ausschließlich die Patientengruppe mit CTO in lediglich einem Koronargefäß Berücksichtigung.

\subsection{1 $\mathrm{CTO}_{\text {NIRA }}-$ Lokalisation}

Von den 123 Patienten mit $\mathrm{CTO}_{\text {NIRA }}$ konnte bei 108 Patienten ein $\mathrm{CTO}_{\text {NIRA }}$ in ausschließlich einem Koronargefäß nachgewiesen werden, dies waren 8,7 \% aller STEMIPatienten und 87,8 \% aller Patienten mit $\mathrm{CTO}_{\mathrm{NIRA}}$. Bei 15 Patienten wurde mehr als ein von einem CTO $_{\text {NIRA }}$ betroffenes Gefäß diagnostiziert: 13 Patienten $(1 \%$ aller STEMIPatienten) hatten zusätzlich zum Infarktgefäß zwei weitere chronisch verschlossene epikardiale Gefäße, bei zwei Patienten (0,2 \% aller STEMI-Patienten) waren sogar alle drei großen Koronararterien chronisch verschlossen (Abbildung 23). 


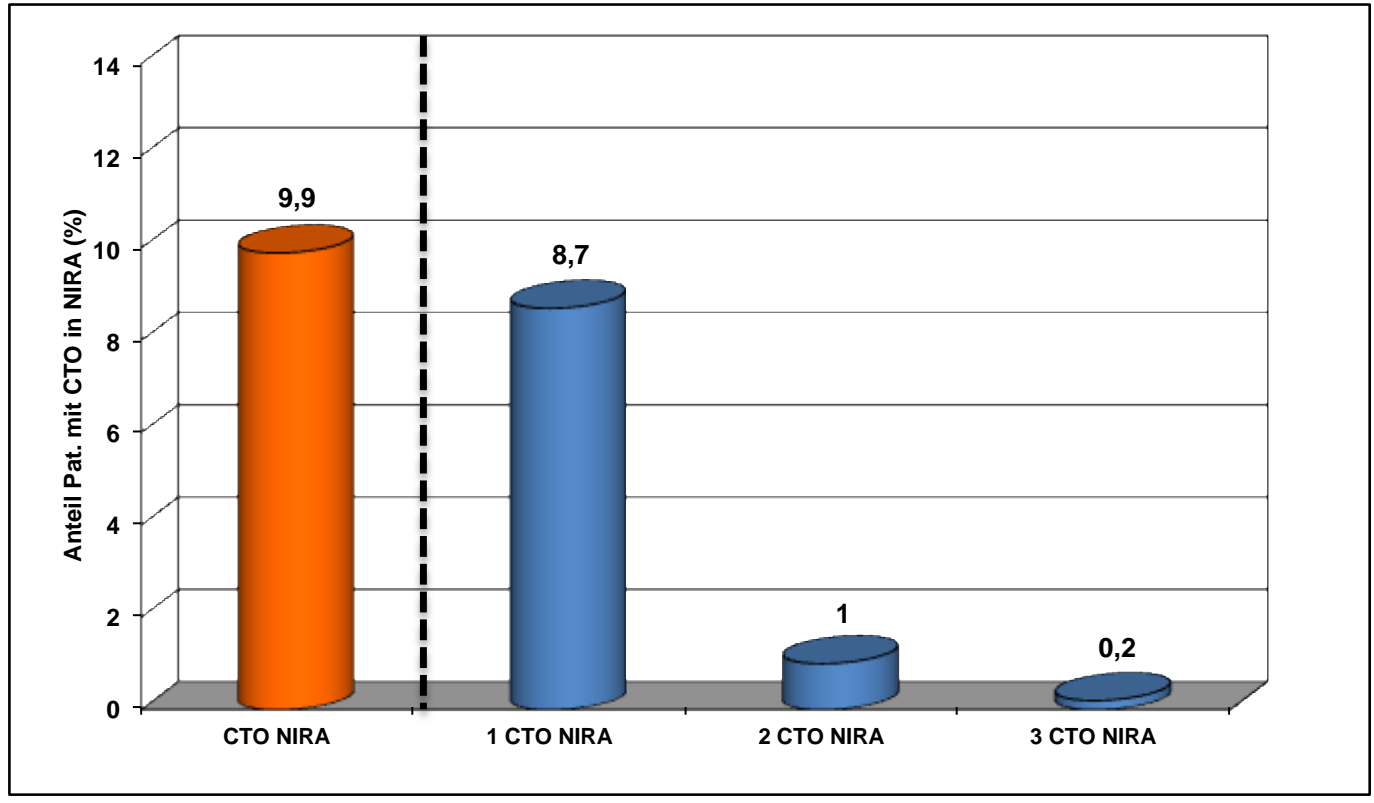

Abbildung 23: Anteil Patienten mit $\mathrm{CTO}_{\text {NIRA }}$ an der Gesamtgruppe ( $\mathrm{n}=1.247$ Patienten mit STEMI)

Betrachtet man alle 123 Patienten mit $\mathrm{CTO}_{\text {NIRA }}$ fällt auf, dass bei $50 \%$ der Patienten $(\mathrm{n}=62)$ ein chronischer Verschluss in der rechte Koronararterie lokalisiert war, bei $28 \%$ $(n=34)$ fand sich ein CTO $_{\text {NIRA }}$ im RIVA und bei $36 \%(n=44)$ im RCX. Da in diesem Fall auch die Patienten mit $>1 \mathrm{CTO}_{\text {NIRA }}$ berücksichtigt wurden, gibt es, wie bereits erwähnt, mehr betroffene Gefäße als Patienten. Abbildung 24 präsentiert daher selektiert die betroffenen Gefäße bei allen STEMI-Patienten mit ausschließlich einem $\mathrm{CTO}_{\mathrm{NIRA}^{-}}$-Gefäß $(\mathrm{n}=108)$. Auch hier zeigte sich, dass die RCA mit $48 \%(\mathrm{n}=52)$ am häufigsten von einem CTO $_{\text {NIRA }}$ betroffen war (RIVA: $21 \%, \mathrm{n}=23$; RCX: $31 \%, \mathrm{n}=33$ ).

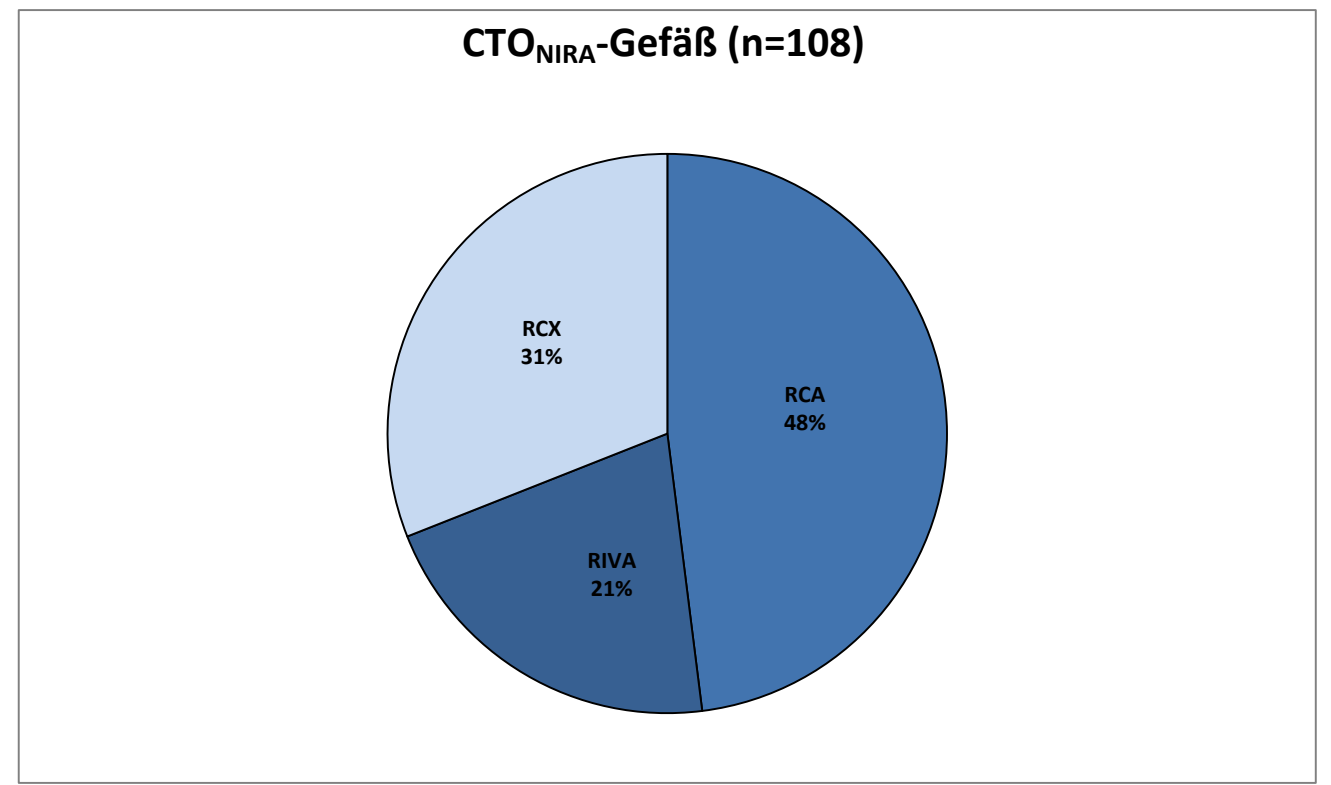

Abbildung 24: Anteil CTO NIRA $_{-}-$Gefäß (Alle Patienten mit $1 \mathrm{CTO}_{\mathrm{NIRA}}, \mathrm{n}=108$ ) 
Die Basischarakteristika der STEMI-Patienten aufgegliedert nach der CTO $_{\text {NIRA-Lokalisation }}$ befinden sich in Tabelle $17 \mathrm{im}$ Anhang (siehe Kapitel 7.4). Signifikante Unterschiede zwischen den einzelnen CTO-Lokalisationen fanden sich dabei lediglich beim Patientenalter (Patienten mit $\mathrm{CTO}_{\text {NIRA }}$ in der RCA waren deutlich jünger), im Anteil an der Patientenaufnahme während der Rufbereitschaft (Patienten mit CTO $_{\text {NIRA }}$ im RIVA wurden deutlich seltener während der Rufbereitschaft aufgenommen) und beim Anteil am jeweiligen Koronarversorgungstyp (siehe Kapitel 4.3.2). Die signifikanten Unterschiede hinsichtlich der Infarktlokalisation und damit einhergehend des Infarktgefäßes waren nicht aussagekräftig, da je nach Lokalisation das $\mathrm{CTO}_{\text {NIRA }}-$ Gefäß nicht als akutes STEMI-Gefäß infrage kommen konnte.

Zur detaillierteren Betrachtung wurden die vom $\mathrm{CTO}_{\text {NIRA }}$ betroffenen Gefäße in einzelne Segmente eingeteilt und demnach die Verschlusslokalisation gesondert beurteilt (siehe Kapitel 3.5). Am häufigsten fand sich dabei insgesamt ein $\mathrm{CTO}_{\text {NIRA }}$ in der proximalen RCA $\left(30 \%\right.$ aller $\left.\mathrm{CTO}_{\text {NIRA }}\right)$. Weitere häufige Verschlusslokalisationen waren daneben die mediale RCA (11\%) sowie der proximale RCX (10\%) und der proximalen RIVA (9\%).

Bei den 52 Patienten mit $\mathrm{CTO}_{\text {NIRA }}$ in der RCA befanden sich $96 \%(\mathrm{n}=50)$ der Verschlüsse in den Koronarsegmenten $1(62 \%, \mathrm{n}=32), 2(23 \%, \mathrm{n}=12)$ und $3(12 \%$, $\mathrm{n}=$ 6). Jeweils ein Patient wies einen $\mathrm{CTO}_{\mathrm{NIRA}}$ in Segment 4 bzw. 16 auf (Abbildung 25).

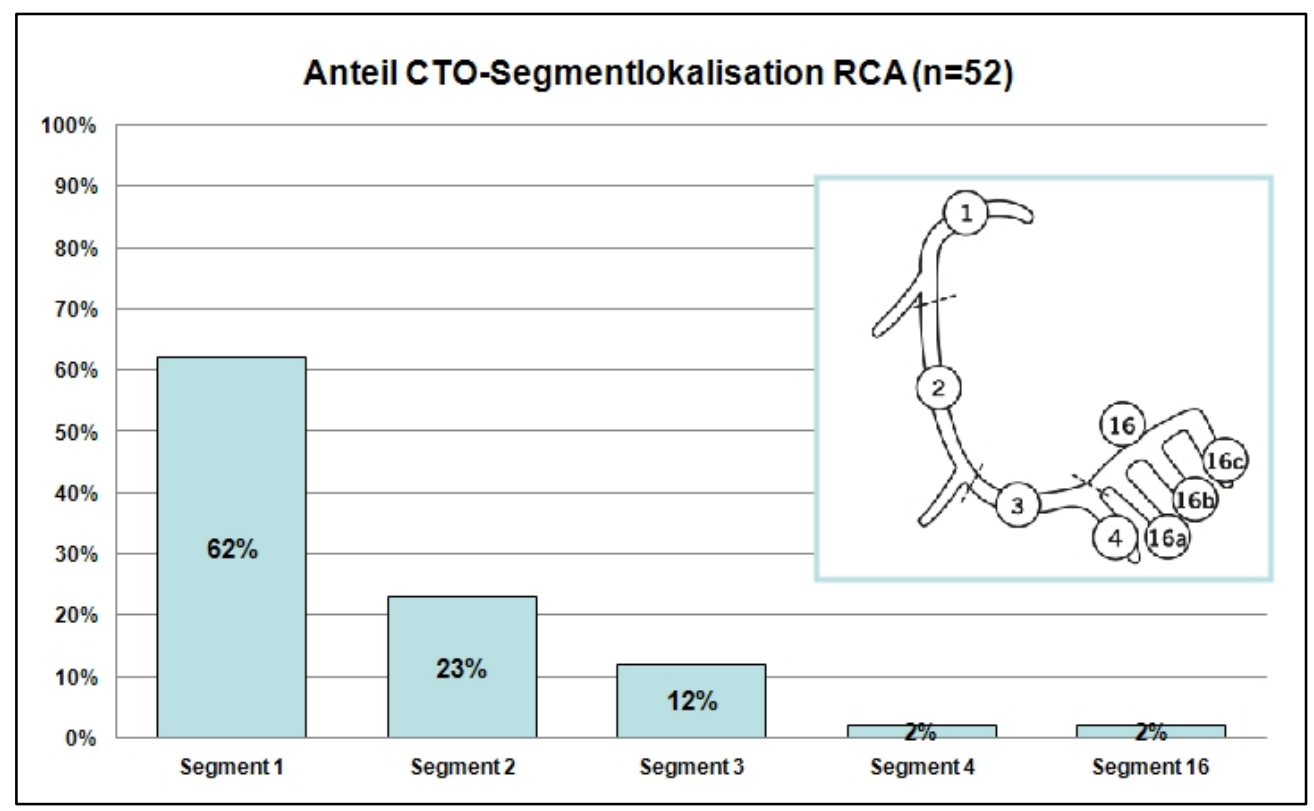

Abbildung 25: Anteil der CTO-Segmentlokalisation in der RCA (Alle Patienten mit $\mathrm{MVD}+1 \mathrm{CTO}_{\mathrm{NIRA}}$ in der RCA, $\mathrm{n}=52$ )

Von den 23 Patienten, bei denen ein $\mathrm{CTO}_{\text {NIRA }}$ im RIVA diagnostiziert wurde, befand sich der chronische Verschluss bei $82 \%(\mathrm{n}=19)$ der Patienten in Segment $6(43 \%, \mathrm{n}=10)$ 
und $7(39 \%, \mathrm{n}=9)$. Bei jeweils zwei Patienten war der $\mathrm{CTO}_{\text {NIRA }}$ in Segment 8 bzw. 9 lokalisiert (Abbildung 26).

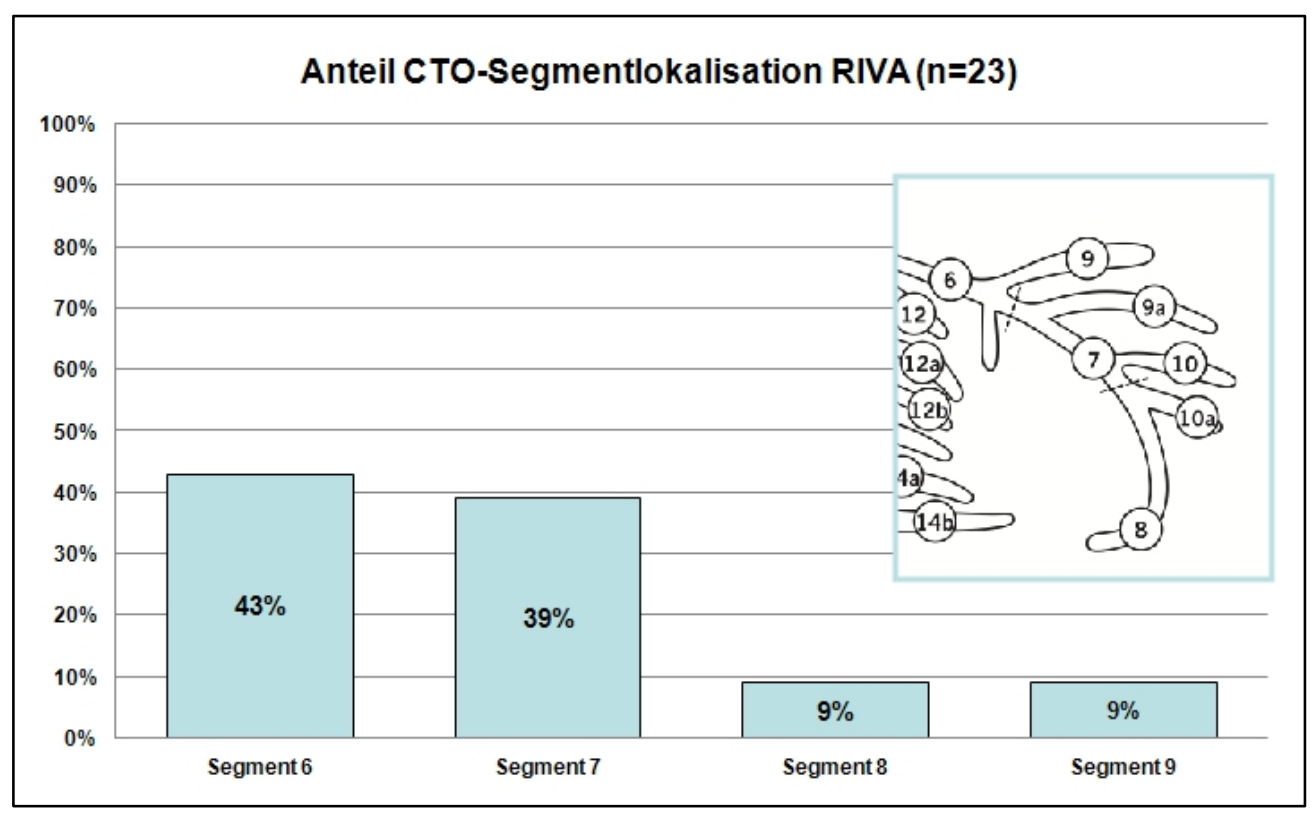

Abbildung 26: Anteil der CTO-Segmentlokalisation im RIVA (Alle Patienten mit MVD+1CTO ${ }_{\text {NIRA }}$ im RIVA, $\mathrm{n}=23$ )

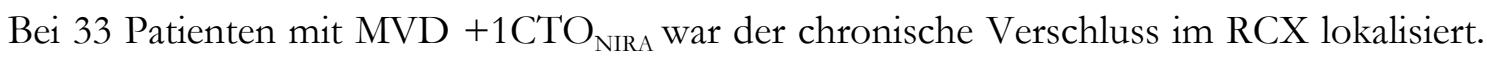
In $94 \%$ der Fälle lag der CTO in den Segmenten $11(33 \%, \mathrm{n}=11), 13(30 \%, \mathrm{n}=10)$ oder $14(30 \%, \mathrm{n}=10)$. Bei zwei Patienten $(6 \%)$ konnte ein CTO im Segment 14a nachgewiesen werden (Abbildung 27).

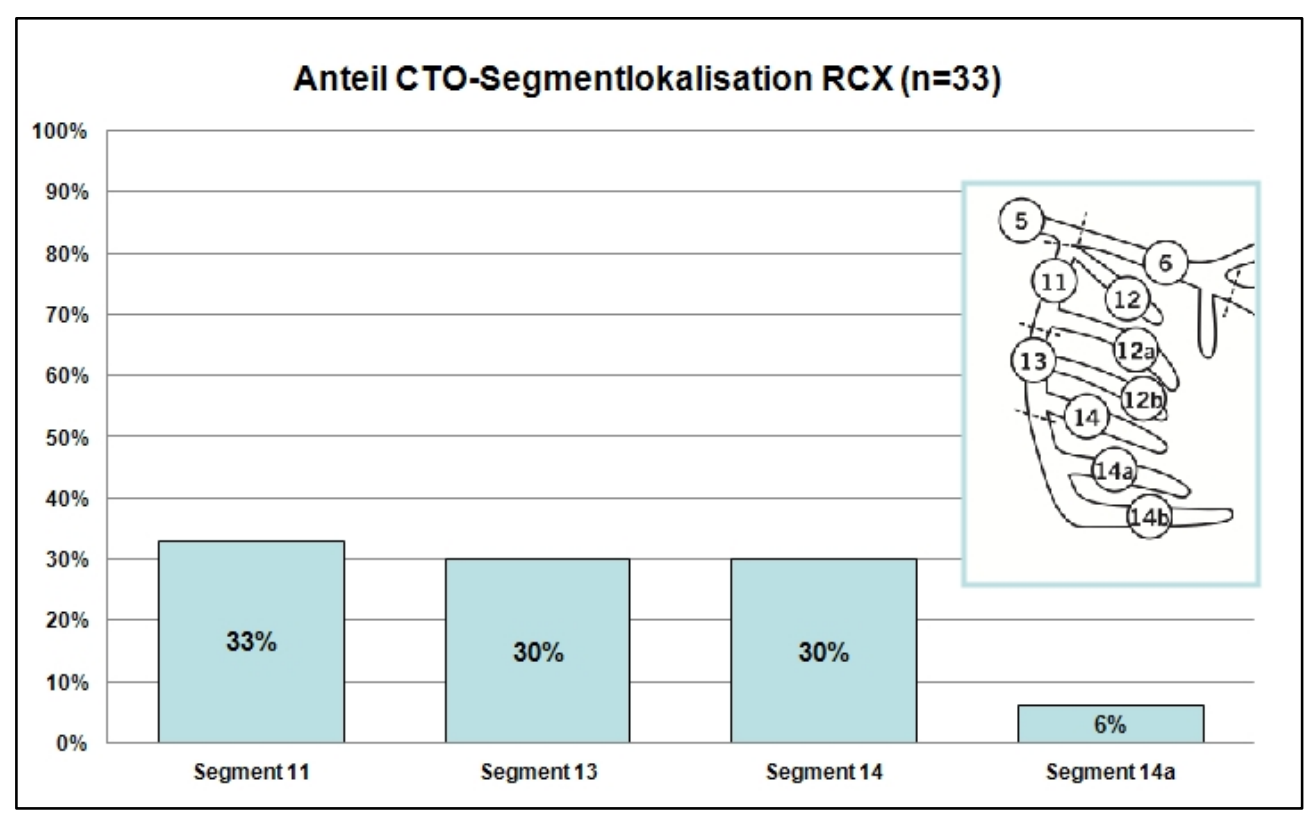

Abbildung 27: Anteil der CTO-Segmentlokalisation im RCX (Alle Patienten mit MVD+1CTO ${ }_{\text {NIRA }}$ im RCX, $\mathrm{n}=33$ ) 
Unabhängig vom betroffenen Koronargefäß befand sich in der Gruppe MVD +1CTO ${ }_{\text {NIRA }}$ ( $\mathrm{n}=108)$ annähernd jeder zweite chronische Verschluss in einem proximalen Gefäßabschnitt (Segment 1, 5, 6 oder 11; $49 \%, \mathrm{n}=53$ ). Mit zunehmender Distanz zum Koronarostium traten auch weniger chronische Verschlüsse auf (Abbildung 28).

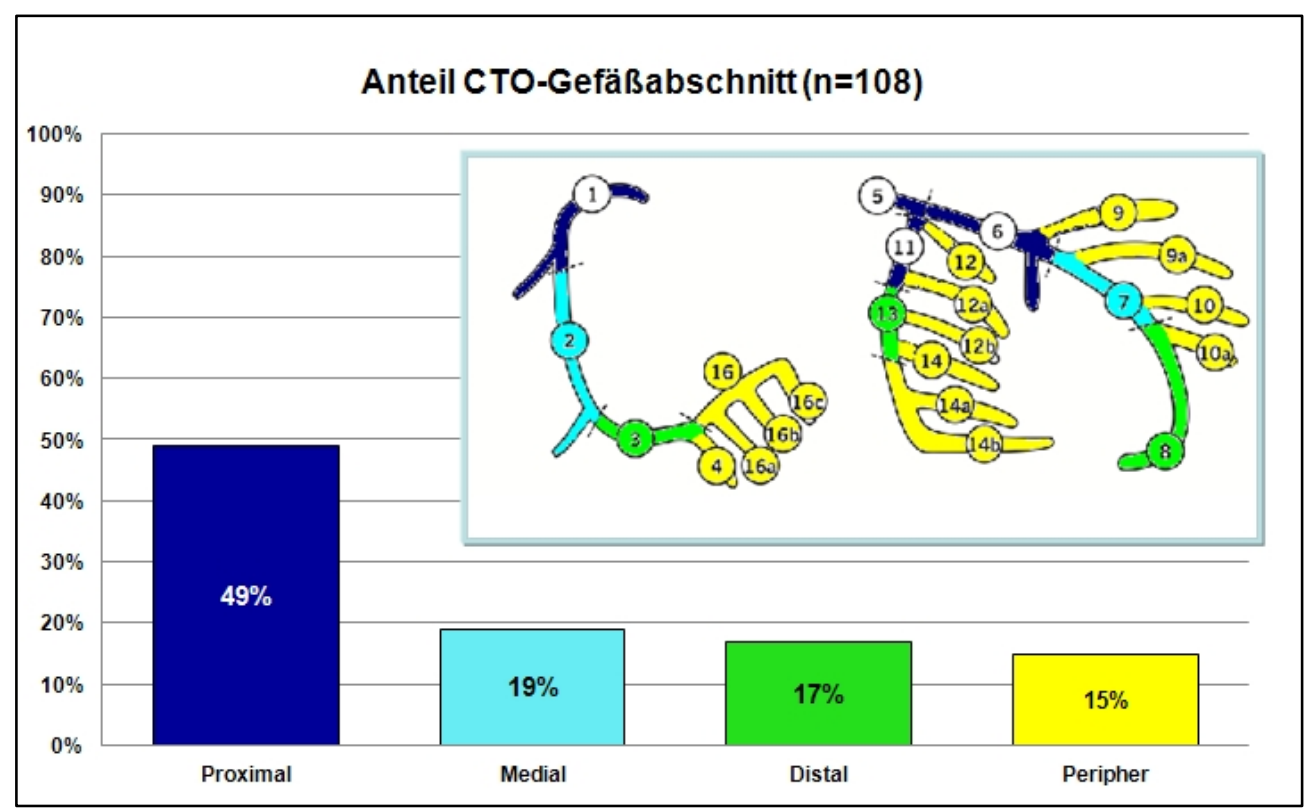

Abbildung 28: Anteil CTO NIRA -Abschnitt (Alle Patienten mit $1 \mathrm{CTO}_{\mathrm{NIRA}}, \mathrm{n}=108$ )

\subsubsection{Versorgungstyp}

Ein Linksversorgungstyp konnte bei $10 \%(\mathrm{n}=11)$ aller STEMI-Patienten mit ausschließlich einem $\mathrm{CTO}_{\text {NIRA }}$ koronarangiographisch nachgewiesen werden (Definition siehe Kapitel 3.5). Demgegenüber wurde bei 97 Patienten (90\%) die Koronarversorgung definitionsgemäß als Rechtsversorgungstyp bezeichnet (Abbildung 29).

Mit Blick auf den Koronarversorgungstyp fanden sich bezüglich des vom CTO betroffenen Gefäßes statistisch signifikante Unterschiede ( $p=0,01)$. Während $19 \%$ aller Patienten mit $\mathrm{CTO}_{\mathrm{NIRA}}$ in der RCA einen Linksversorgungstyp aufwiesen, waren es bei den Patienten mit $\mathrm{CTO}_{\text {NIRA }}$ im RIVA lediglich $4 \%$. Von den 33 Patienten mit CTO $_{\text {NIRA }}$ im RCX konnte darüber hinaus bei keinem Patienten ein Linksversorgungstyp nachgewiesen werden. 


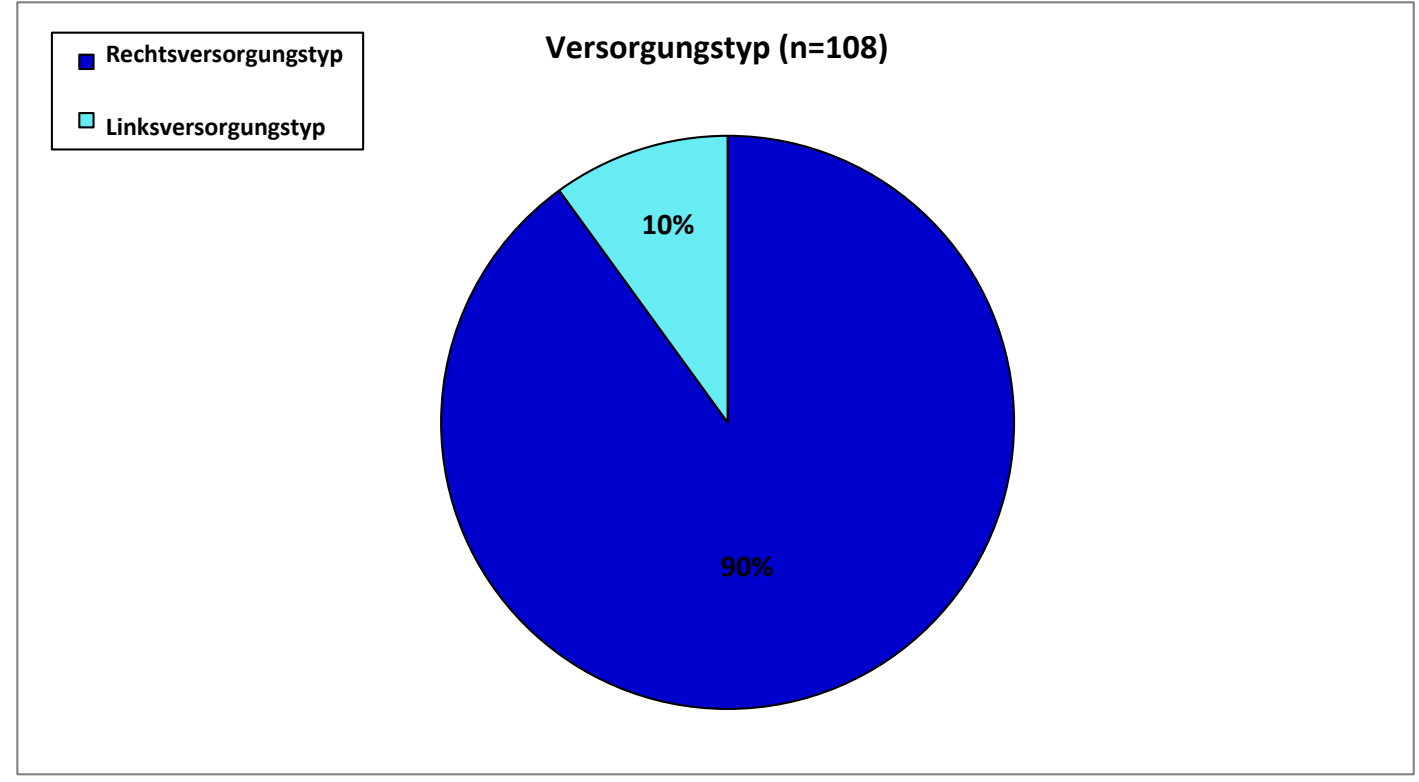

Abbildung 29: Versorgungstyp (Alle Patienten mit $1 \mathrm{CTO}_{\mathrm{NIRA}}, \mathrm{n}=108$ )

\subsubsection{Kollateralstatus}

Von den 108 Patienten mit 1 CTO $_{\text {NIRA }}$ konnte angiographisch bei 92 Patienten (85\%) eine relevante Kollateralisierung (Definition siehe Kapitel 3.5) der Gefäßabschnitte distal des CTO festgestellt werden (Abbildung 30).

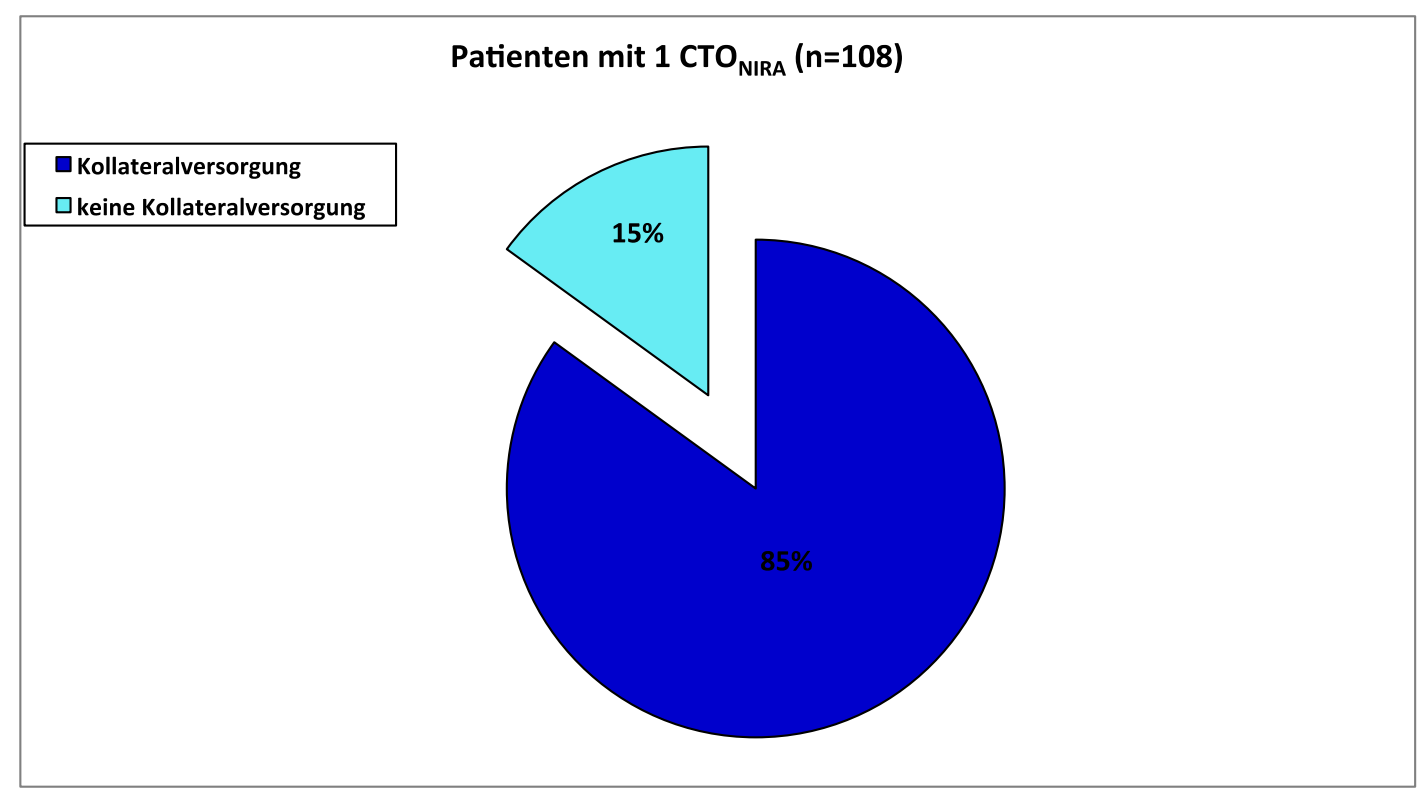

Abbildung 30: Anteil Kollateralversorgung des CTO-Gefäßes (Alle Patienten mit 1 $\left.\mathrm{CTO}_{\text {NIRA }}, \mathrm{n}=108\right)$

Dabei wurden CTOs der RCA mit $90 \%$ am häufigsten angiographisch sichtbar kollateralisiert, demgegenüber wiesen $87 \%$ der CTO $_{\text {NIRA }}$ im RIVA und $76 \%$ der CTO $_{\text {NIRA }}$ im RCX eine sichtbare Kollateralversorgung auf. Am häufigsten bestand eine interarterielle 
Kollateralverbindung zwischen dem RIVA und der chronisch verschlossenen RCA ( $\mathrm{n}=27$ von 52 Patienten mit RCA-CTO ${ }_{\text {NIRA }} ; 52 \%$; s. auch Tabelle 12, Kapitel 4.3.5.6).

Die Unterschiede hinsichtlich des Anteils an Patienten mit Kollateralversorgung zwischen den Gruppen des jeweils betroffenen $\mathrm{CTO}_{\mathrm{NIRA}}$-Gefäßes waren allerdings statistisch nicht signifikant (p-Wert: 0,174).

Von den 92 Patienten mit sichtbarer Kollateralisierung konnte bei 15 Patienten (16\%) sowohl eine Kollateralisierung über intrakoronare Brückenkollateralen als auch über interkoronare Kollateralen beschrieben werden. Während neun Patienten (10\%) ausschließlich Brückenkollateralen aufwiesen, konnten bei den restlichen 68 Patienten (74\%) ausschließlich interkoronare Kollateralen identifiziert werden (Abbildung 31).

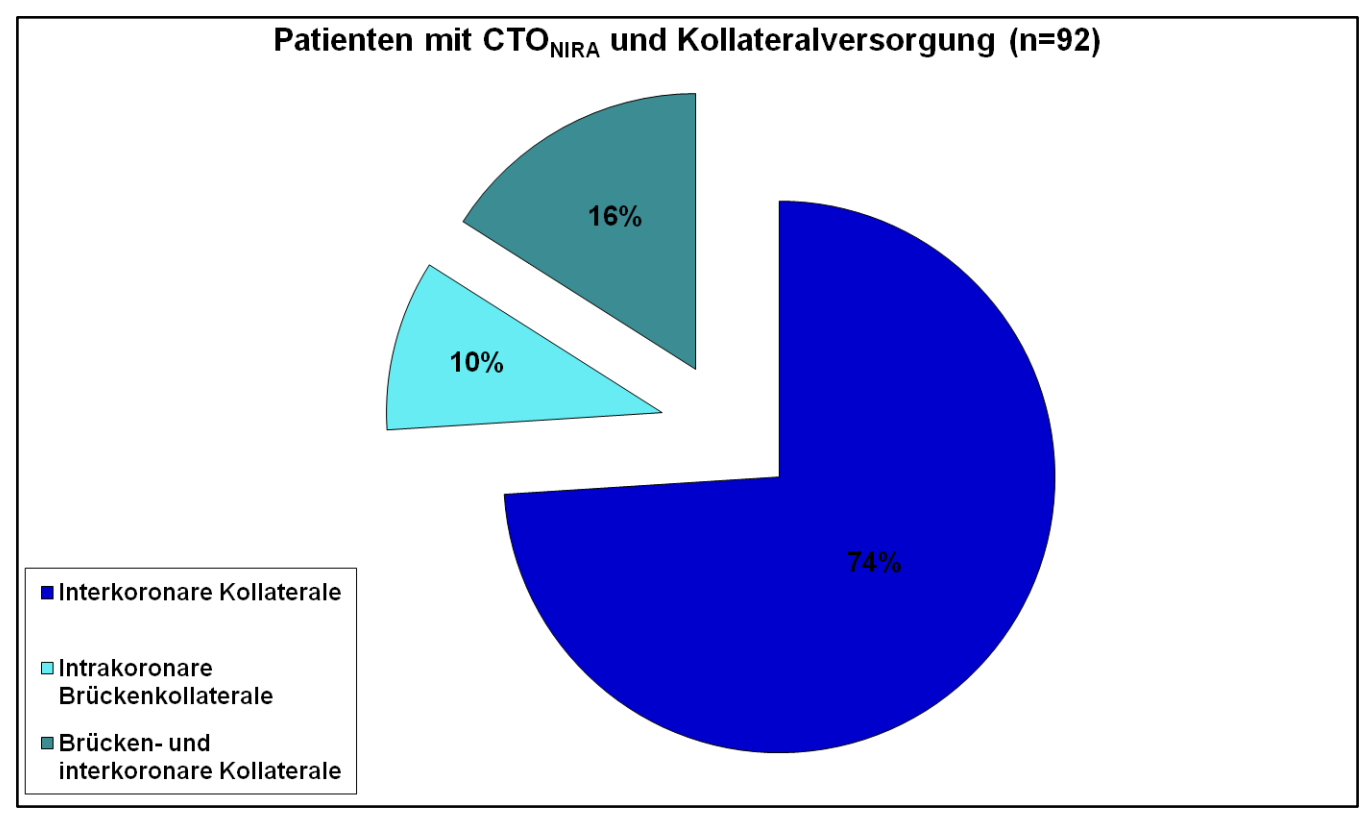

Abbildung 31: Art der Kollateralisierung (Alle Patienten mit $1 \mathrm{CTO}_{\text {NIRA }}$ und Kollateralversorgung, $\mathrm{n}=92$ )

Die Patienten mit interkoronaren Kollateralen wurden darüber hinaus nach dem Ursprungskoronargefäß der Kollaterale klassifiziert, um auch solche Kollateralen zu identifizieren, die ihren Ursprung direkt im vom akuten Infarkt betroffenen Gefäß haben. Da dieses akute Infarktgefäß bei einigen STEMI-Patienten mit $\mathrm{CTO}_{\mathrm{NIRA}}$ und nicht erfolgter primärer PCI nicht zweifelsfrei eruiert werden konnte und zudem auch Kollateralen analysiert wurden, die sich erst nach erfolgreicher PCI darstellten, wurden für die folgenden Untersuchungen nur STEMI-Patienten mit tatsächlich durchgeführter primärer PCI des Infarktgefäßes berücksichtigt $(\mathrm{n}=87)$. Von diesen 87 STEMI-Patienten mit PCI wiesen $75 \%(n=65)$ eine interkoronare Kollateralisierung auf. Bei $51 \%$ dieser Patienten $(n=33)$ entsprang die Kollaterale direkt aus der vom akuten STEMI betroffenen Koronararterie (IRA = infart-related artery; Abbildung 32). 


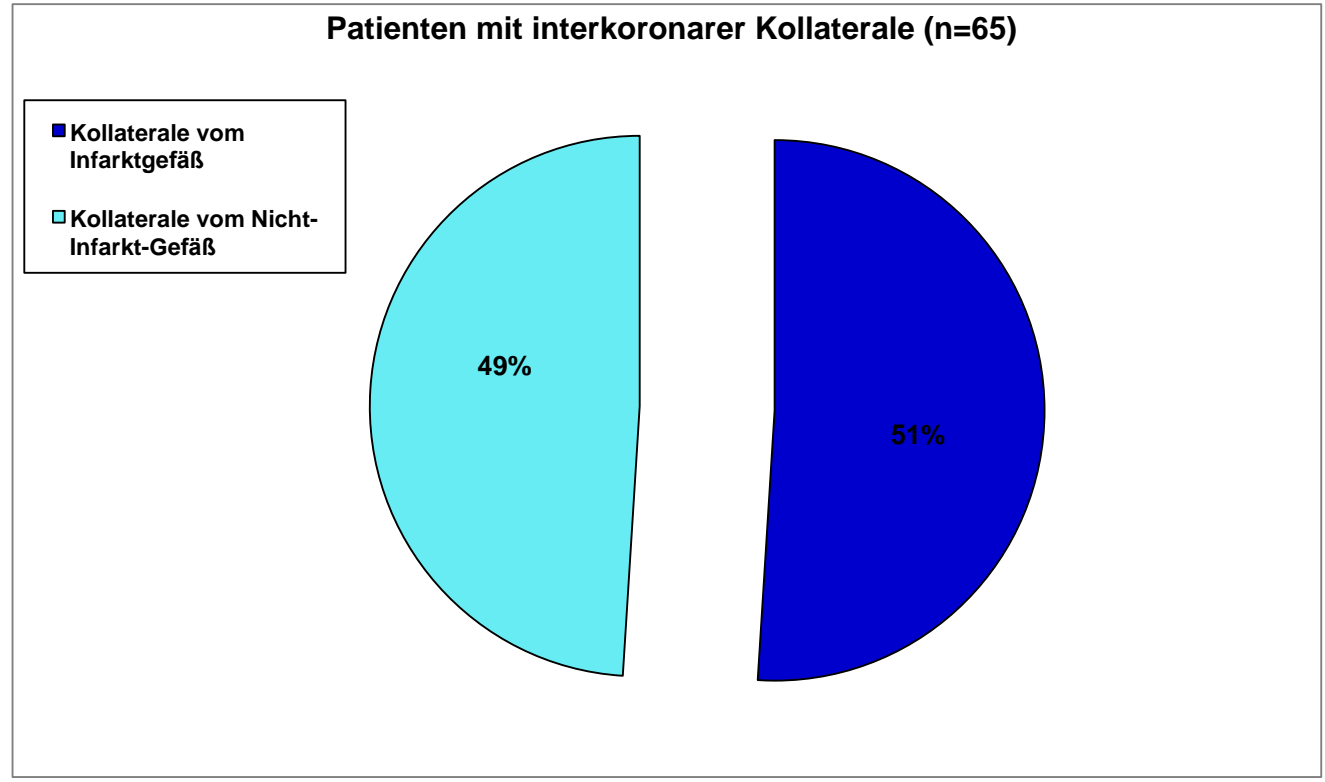

Abbildung 32: Herkunft der interkoronaren Kollaterale (Alle STEMI-Patienten mit PCI und $1 \mathrm{CTO}_{\text {NIRA }}$ sowie interkoronarer Kollateralisierung, $\mathrm{n}=65$ )

Bei den 33 PCI-Patienten mit Kollateralisierung des CTO über das Infarktgefäß wurde darüber hinaus die Abgangsstelle des Kollateralgefäßes in Bezug zum akuten Verschluss untersucht. Bei 18 Patienten (55 \% dieser Fälle) entsprang die Kollaterale proximal des akuten Verschlusses. Die restlichen 15 Patienten (45\%) wiesen einen Kollateralursprung distal des akuten Verschlusses auf, die Kollaterale wurde somit erst nach erfolgreicher PCI mit wiederhergestelltem Koronarfluss sichtbar (Abbildung 33).

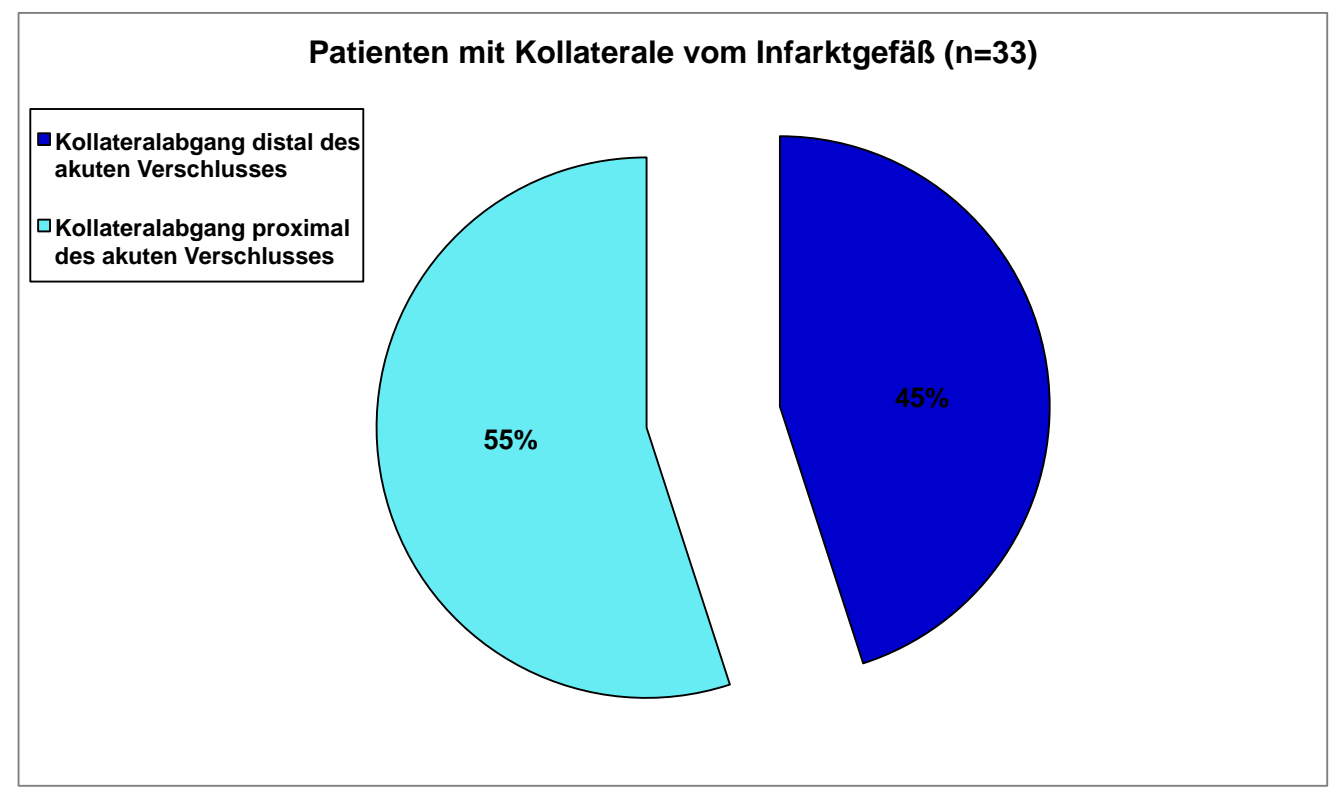

Abbildung 33: Kollateralabgang in Bezug zum akuten Verschluss (Alle STEMI-Patienten mit PCI und $1 \mathrm{CTO}_{\text {NIRA }}$ sowie Kollateralversorgung über das Infarktgefäß, n = 33) 


\subsubsection{Hinweise auf einen früheren Myokardinfarkt}

Wie in Kapitel 1.3.3 beschrieben, deutet die Studienlage darauf hin, dass ein Großteil der chronischen Koronargefäßverschlüsse aus akuten Infarkten hervorgeht. Diesbezüglich wurden alle Patienten mit $\mathrm{CTO}_{\text {NIRA }}$ auf die Zeichen eines abgelaufenen Infarktes hin untersucht. Von Bedeutung waren hierbei vor allem pathologische Q-Zacken im EKG (siehe Kapitel 3.6) sowie anamnestisch ein Infarkt in der Vorgeschichte. Von den 108 Patienten mit ausschließlich $1 \mathrm{CTO}_{\text {NIRA }}$ konnten bei 36 Patienten (33\%) pathologische QZacken in dem zum $\mathrm{CTO}_{\text {NIRA }}$ passenden Areal nachgewiesen werden. Einen alten Infarkt in der Vorgeschichte gaben 18 Patienten (17\%) an. Zusammengenommen waren dies insgesamt 44 Patienten (41\%), die entweder eine pathologische Q-Zacke, einen Infarkt in der Vorgeschichte oder beide Eigenschaften zusammen aufwiesen. Es fällt auf, dass bei ca. $50 \%$ der Patienten mit $\mathrm{CTO}_{\mathrm{NIRA}}$ in RCA und RIVA ein Hinweis auf einen alten Infarkt zu finden war. Indes wies nur jeder fünfte STEMI-Patient mit CTO $_{\text {NIRA }}$ im RCX einen Hinweis auf einen alten Infarkt auf (Tabelle 6).

Tabelle 6: Anteil der Patienten mit Hinweis auf einen abgelaufenen, früheren Infarkt (Alle Patienten mit $\left.1 \mathrm{CTO}_{\mathrm{NIRA}}, \mathrm{n}=108\right)$

\begin{tabular}{|c|c|c|c|c|c|}
\hline & $\begin{array}{c}\text { Gesamt } \\
(\mathbf{n}=108)\end{array}$ & $\begin{array}{c}\text { CTO } \\
\text { RCA (n = 52) }\end{array}$ & $\begin{array}{c}\text { CTO }_{\text {NIRA in }} \text { RIVA (n = 23) } \\
\text { RIVA }\end{array}$ & $\begin{array}{c}\text { CTO }_{\text {NIRA in }} \\
\text { RCX (n = 33) }\end{array}$ & p-Wert \\
\hline $\begin{array}{c}\text { Pathologische Q- } \\
\text { Zacken }\end{array}$ & $36(33 \%)$ & $20(38 \%)$ & $12(52 \%)$ & $4(12 \%)$ & 0,004 \\
\hline $\begin{array}{c}\text { Infarkt in } \\
\text { Vorgeschichte }\end{array}$ & $18(17 \%)$ & $10(19 \%)$ & $3(13 \%)$ & $5(15 \%)$ & 0,772 \\
\hline $\begin{array}{c}\text { Q-Zacke } \\
\text { und } \\
\text { in Voder Infarkt }\end{array}$ & $44(41 \%)$ & $25(48 \%)$ & $12(52 \%)$ & $7(21 \%)$ & 0,222 \\
\hline
\end{tabular}

Bei $72 \%$ der Patienten mit pathologischen Q-Zacken befand sich der CTO $_{\text {NIRA }}$ im proximalen bzw. medialen Segment des RIVA oder der RCA. Bezüglich der Patienten mit und ohne Hinweis auf einen abgelaufenen, früheren Infarkt gab es mit Blick auf den Anteil der Patienten mit Kollateralversorgung keine Unterschiede (84 \% vs. 85 \%).

\subsubsection{Kurzzeitsterblichkeit und Patientenrisiko}

Bezugnehmend auf Kapitel 4.2.7 lag die Krankenhaussterblichkeit von STEMI-Patienten mit MVD $+1 \mathrm{CTO}_{\text {NIRA }}$ bei 25,9\%. Die 30-Tage Sterblichkeit in dieser Patientengruppe lag bei 26,9\%. Im Folgenden werden aus diesem Kollektiv einige Untergruppen mit einem besonders hohen Sterblichkeitsrisiko präsentiert. 
4.3.5.1 Kurzzeitsterblichkeit und TIMI Risk Score abhängig vom betroffenen CTO-Gefäß Abbildung 34 präsentiert die Krankenhaussterblichkeit in Abhängigkeit vom CTO-Gefäß. Es wird deutlich, dass die Krankenhaussterblichkeit bei STEMI-Patienten mit einem $\mathrm{CTO}_{\text {NIRA }}$ in der RCA mit 30,8 \% ( $\left.\mathrm{n}=16\right)$ am höchsten war. Dabei ließ sich hinsichtlich der Krankenhaussterblichkeit zwischen dem RIVA (21,7 \%, $\mathrm{n}=5)$ und dem RCX (21,2\%, $\mathrm{n}=7)$ kein Unterschied feststellen. Nach 30-Tagen blieb die Sterblichkeit in den Gruppen mit $\mathrm{CTO}_{\text {NIRA }}$ im RIVA mit 21,7 \% und im RCX mit 21,2 \% unverändert. Von den Patienten mit $\mathrm{CTO}_{\text {NIRA }}$ in der RCA verstarb innerhalb von 30-Tagen ein weiterer Patient, die 30-Tage Sterblichkeit lag in dieser Gruppe bei 32,7\%. Allerdings sind die Sterblichkeitsunterschiede, mutmaßlich aufgrund der zu geringen Patientenzahlen in den Untergruppen, statistisch nicht signifikant $(p=0,541)$.

Trotz niedrigster Sterblichkeit wiesen Patienten mit einem CTO $_{\text {NIRA }}$ im RCX im Durchschnitt mit 4,97 Punkten den höchsten TIMI Risk Score auf, dieser betrug bei Patienten mit CTO $_{\text {NIRA }}$ in der RCA 4,90 Punkte und bei Patienten mit CTO $_{\text {NIRA }}$ im RIVA 4,61 Punkte.

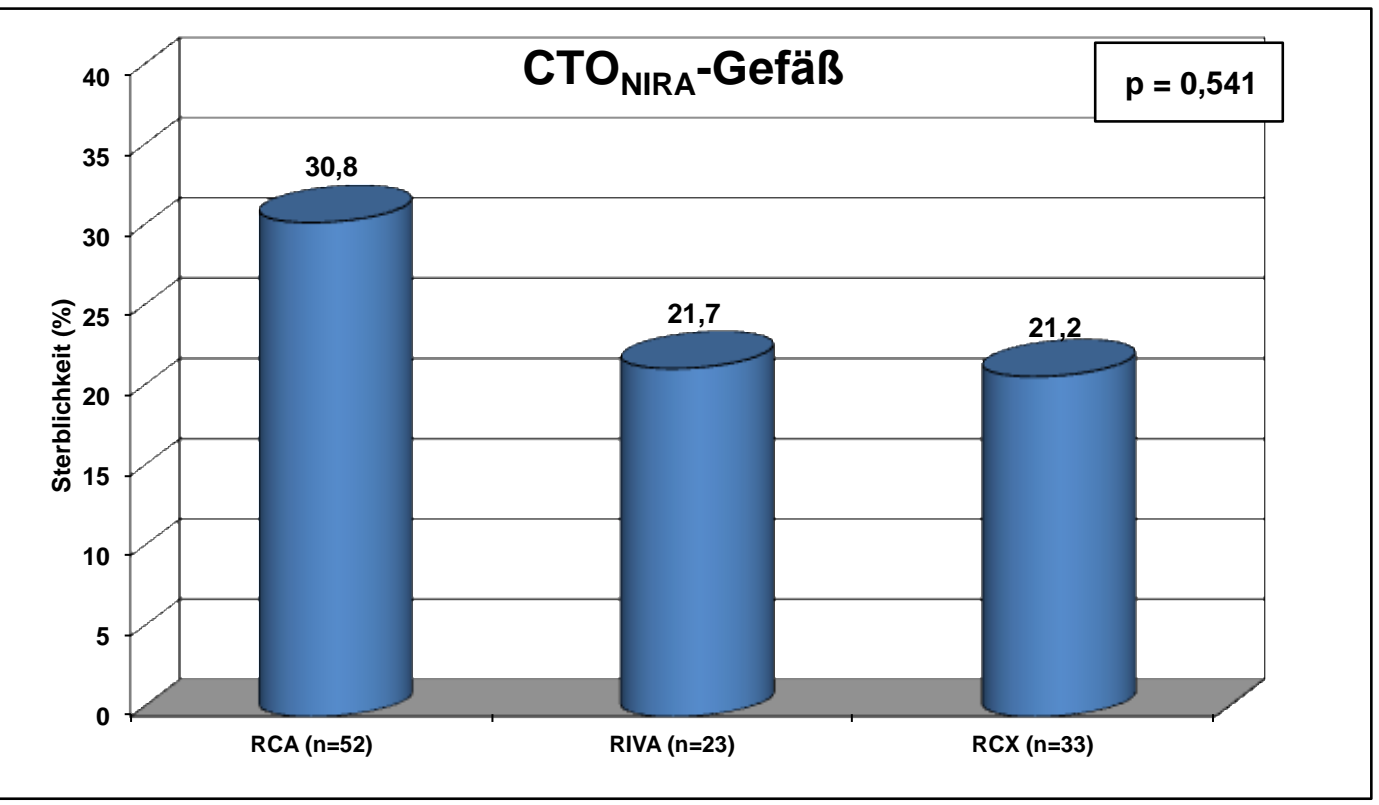

Abbildung 34: Krankenhaussterblichkeit (\%) in Abhängigkeit vom CTO-Gefäß

Abbildung 15 (Kapitel 4.2.5) zeigt, dass Patienten mit MVD $+\mathrm{CTO}_{\mathrm{NIRA}}$ ein deutlich erhöhtes Schockrisiko aufwiesen. In der Gruppe der Patienten mit MVD+1CTO erlitten $31 \%$ einen kardiogenen Schock. Dieses Risiko verteilte sich jedoch nicht gleichmäßig über die betroffenen Koronargefäße. So fällt beim Betrachten dieser Patienten auf, dass ein CTO $_{\text {NIRA }}$ in der RCA mit einem deutlich höheren, aber statistisch nicht signifikanten ( $\mathrm{p}=0,771)$, Risiko für einen kardiogenen Schock einherging $(35 \%, \mathrm{n}=18)$ als ein $\mathrm{CTO}_{\mathrm{NIRA}}$ in RIVA $(30 \%, \mathrm{n}=7)$ bzw. $\operatorname{RCX}(27 \%, \mathrm{n}=9$; Abbildung 35$)$. 


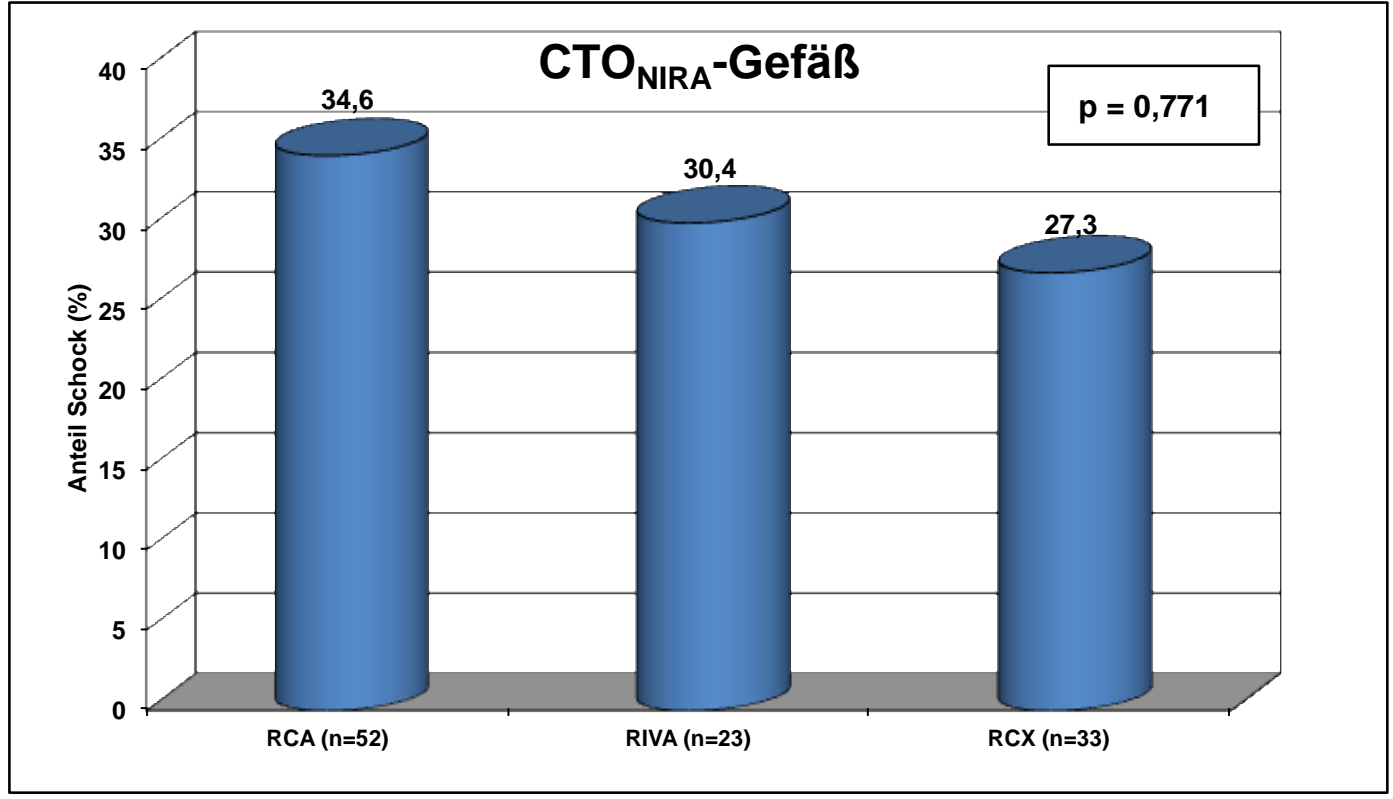

Abbildung 35: Anteil der Patienten mit Schock (\%) in Abhängigkeit vom CTO-Gefäß

Zudem wiesen diese Patienten mit $\mathrm{CTO}_{\mathrm{NIRA}}$ in der RCA im Falle eines kardiogenen Schocks im Vergleich zu den anderen Koronargefäßen eine deutlich, aber ebenfalls nicht signifikant erhöhte Krankenhaussterblichkeit $(66,7 \%, \mathrm{n}=12)$ auf (Abbildung 36).

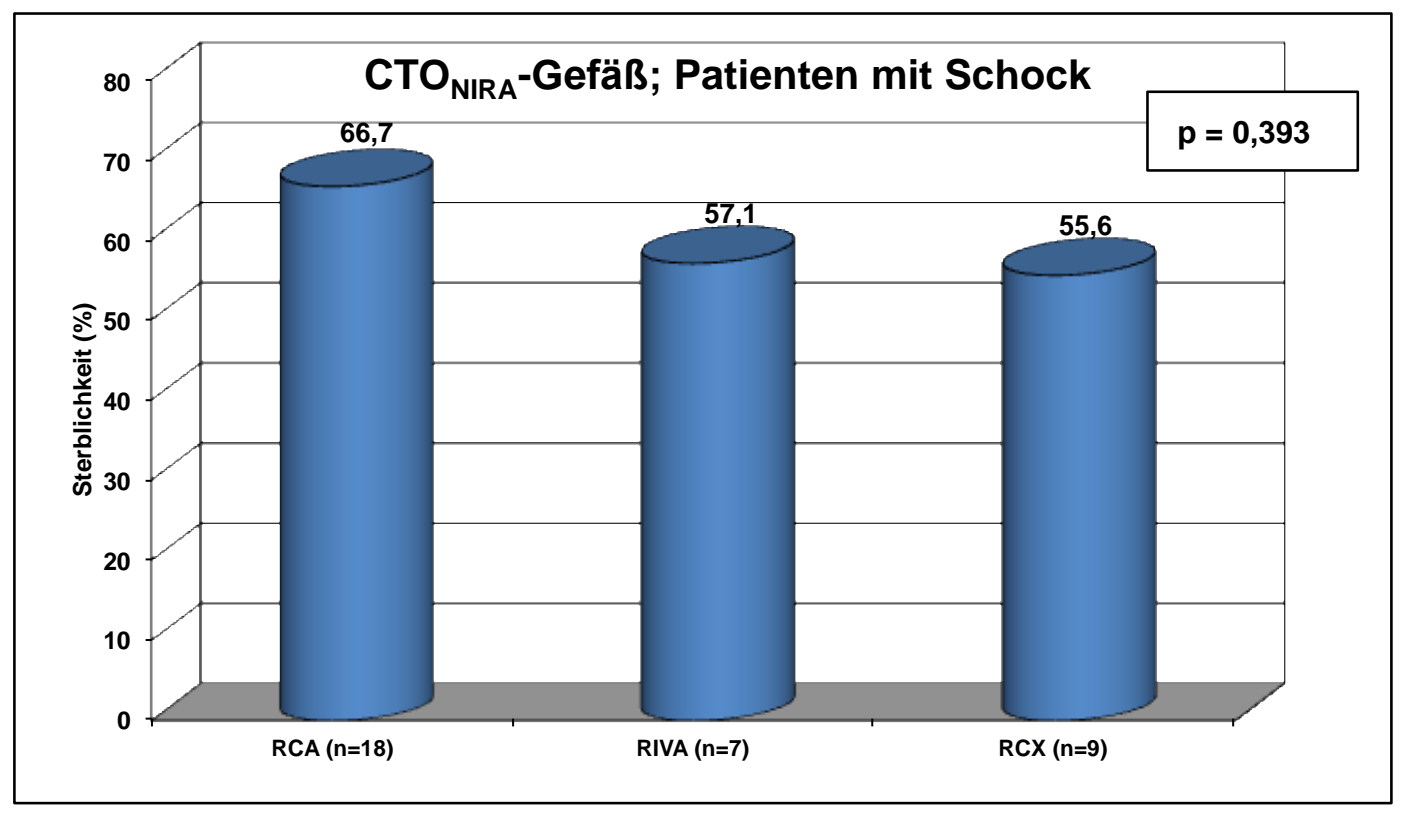

Abbildung 36: Krankenhaussterblichkeit (\%) in Abhängigkeit vom CTO-Gefäß bei Patienten mit Schock und $1 \mathrm{CTO}_{\text {NIRA }}(\mathrm{n}=34)$

\subsubsection{Kurzzeitsterblichkeit abhängig vom akut betroffenen STEMI-Gefäß}

In Abbildung 37 wird die Krankenhaussterblichkeit bei STEMI-Patienten mit 1CTO NIRA mit Blick auf das vom akuten Infarkt betroffene Koronargefäß (culprit lesion) dargestellt. Zu Beachten ist hierbei allerdings, dass das akute Infarktgefäß nur bei Patienten mit erfolgter primärer PCI zweifelsfrei identifiziert werden konnte, sodass im Folgenden bei 
Betrachtung des akuten Infarktgefäßes nur die STEMI-Patienten mit $1 \mathrm{CTO}_{\text {NIRA }}$ und durchgeführter primärer PCI berücksichtigt wurden $(\mathrm{n}=87)$. Unter den Patienten mit nicht erfolgter primärer PCI (Gründe dafür u. a.: kein Zielgefäß, Akut-Bypass-OP) befanden sich einige STEMI-Patienten, bei denen zwar die Lokalisation des chronischen Verschlusses exakt bestimmt werden konnte, aufgrund der Schwere der Gefäßerkrankung war es hier allerdings nicht immer möglich, das akute Infarktgefäß eindeutig zuzuordnen.

Mit Blick auf dieses Patientenkollektiv konnte gezeigt werden, dass bei Vorliegen eines $\mathrm{CTO}_{\text {NIRA }}$ vor allem akute Infarkte des RIVA sowie des RCX mit einer deutlich erhöhten Krankenhaussterblichkeit einhergingen (28,6 \% bzw. 26,3\%). Mit $100 \%$ war dabei die Sterblichkeit bei Patienten mit akutem Verschluss des linken Hauptstammes und zusätzlichem $\mathrm{CTO}_{\text {NIRA }}$ am höchsten. Allerdings befanden sich in dieser Gruppe lediglich zwei Patienten, womit die Aussagekraft dieses Ergebnisses begrenzt ist.

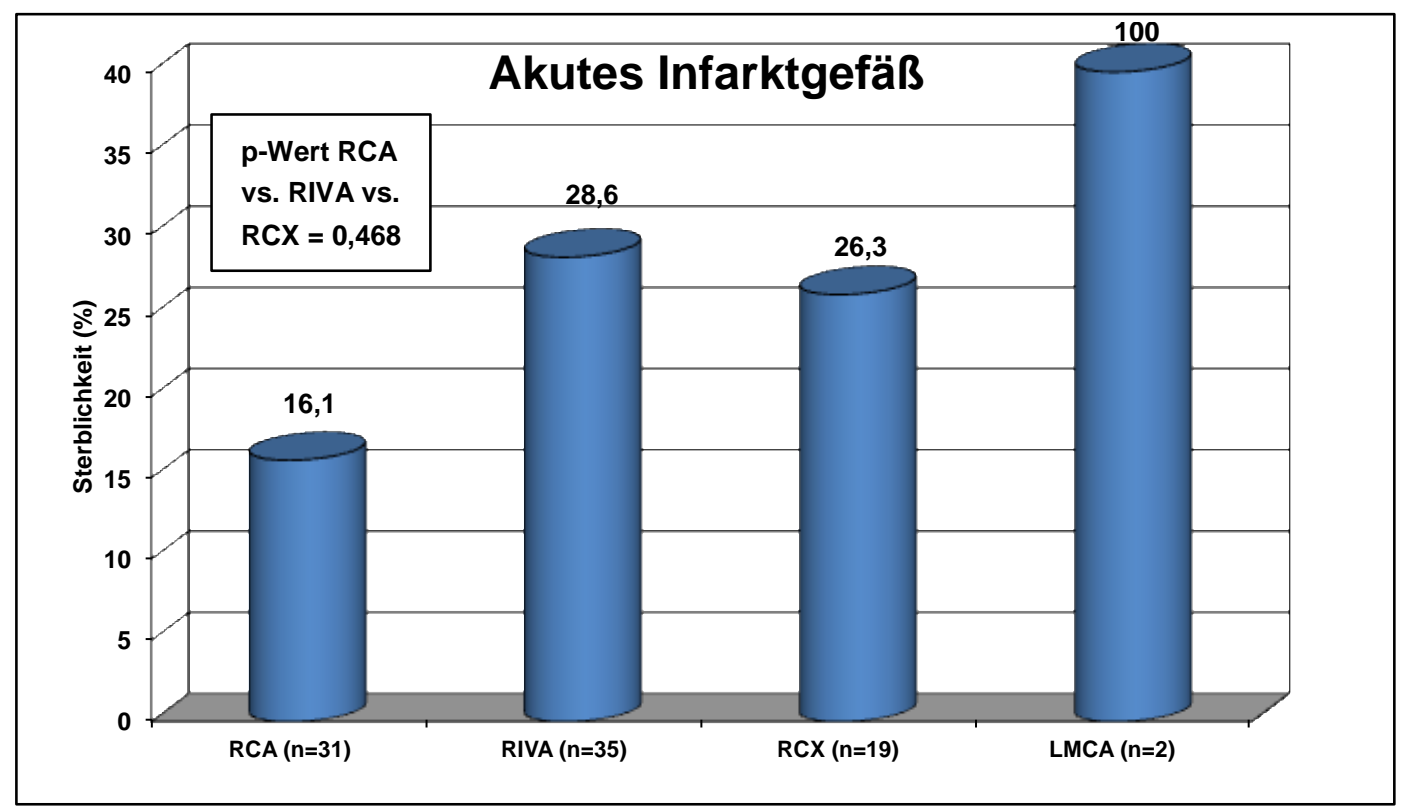

Abbildung 37: Krankenhaussterblichkeit (\%) in Abhängigkeit vom akuten Infarktgefäß (culprit lesion) bei STEMI-Patienten mit PCI und 1CTO NIRA $\left._{(\mathrm{n}}=87\right)$

\subsubsection{Kurzzeitsterblichkeit unter Berücksichtigung von $\mathrm{CTO}_{\text {NIRA }}$-und STEMI- \\ Gefäßlokalisation}

Die folgenden Tabellen präsentieren die Beziehung zwischen dem $\mathrm{CTO}_{\mathrm{NIRA}}-\mathrm{Gefäß}$ und dem akuten Infarktgefäß bei STEMI-Patienten mit PCI. Um den kompletten Koronarstatus jedes Patienten zu berücksichtigen, wurden Patienten mit Rechts- bzw. Linksversorgungstyp gesondert analysiert.

In Tabelle 7 wurden daher nur STEMI-Patienten mit PCI und Rechtsversorgungstyp sowie ausschließlich einem $\mathrm{CTO}_{\text {NIRA }}$ berücksichtigt $(\mathrm{n}=78)$. Auch in dieser Kohorte wiesen Patienten mit $\mathrm{CTO}_{\text {NIRA }}$ in der RCA mit $32 \%$ (10/31 Patienten) eine besonders hohe 
Sterblichkeit innerhalb von 30 Tagen auf. In dieser Gruppe der Patienten mit Rechtsversorgungstyp und $\mathrm{CTO}_{\mathrm{NIRA}}$ der RCA hatten vor allem Patienten mit einem akuten Verschluss des RIVA eine schlechte Prognose, hier verstarben $33 \%$ der Patienten innerhalb von 30 Tagen. Weitere Hochrisikopatienten in der Gruppe der STEMI-Patienten mit Rechtsversorgungstyp waren solche mit einem $\mathrm{CTO}_{\text {NIRA }}$ des RCX in Kombination mit einem STEMI des RIVA (Kurzzeitsterblichkeit: $33 \%$ ) sowie Patienten mit einem CTO $_{\text {NIRA }}$ der RCA in Kombination mit einem STEMI des RCX (Kurzzeitsterblichkeit: $22 \%$ ). Bei zwei Patienten wurde ein Akutverschluss des linken Hauptstamms (LMCA) als Infarktgefäß diagnostiziert, diese beiden Patienten verstarben noch im Krankenhaus.

Beim Betrachten dieser Ergebnisse wird vor allem auch die besondere Bedeutung eines akuten RIVA-Infarktes deutlich. In der vorliegenden Gruppe wiesen Patienten mit akutem STEMI des RIVA und zusätzlichem CTO $_{\text {NIRA }}$ (in RCX oder RCA) mit $33 \%$ (11 von 33 Pat.) ebenfalls eine extrem hohe 30-Tage-Sterblichkeit auf. Akute Verschlüsse des RCX bzw. der RCA zeigten dagegen in dieser speziellen Kohorte der Patienten mit Rechtsversorgungstyp mit einer Sterblichkeit von $17 \%$ (2/12 Patienten) bzw. 16 \% (5/31 Patienten) eine vergleichsweise gute Prognose.

Tabelle 7: Zusammenhang zwischen CTO- und Infarktgefäß mit Blick auf das Patientenoutcome (STEMI-Patienten mit $1 \mathrm{CTO}_{\text {NIRA }}$, Rechtsversorgungstyp und PCI $\mathrm{n}=78$ )

\begin{tabular}{|l|c|c|c|c|c|c|c|c|}
\hline CTO-Gefäß & \multicolumn{3}{|c|}{ RIVA (n= 20) } & \multicolumn{3}{c|}{ RCX (n $=27)$} & \multicolumn{3}{c|}{ RCA (n = 31) } \\
\hline Infarktgefäß & RCA & RCX & LMCA & RIVA & RCA & RIVA & RCX & LMCA \\
\hline Anzahl & 16 & 3 & 1 & 12 & 15 & 21 & 9 & 1 \\
\hline Shock & $2(13 \%)$ & $1(33 \%)$ & $1(100 \%)$ & $3(25 \%)$ & $6(40 \%)$ & $8(38 \%)$ & $3(33 \%)$ & $1(100 \%)$ \\
\hline Tod KHS & $3(19 \%)$ & 0 & $1(100 \%)$ & $4(33 \%)$ & $2(13 \%)$ & $6(29 \%)$ & $2(22 \%)$ & $1(100 \%)$ \\
\hline Tod 30-Tage & $3(19 \%)$ & 0 & $1(100 \%)$ & $4(33 \%)$ & $2(13 \%)$ & $7(33 \%)$ & $2(22 \%)$ & $1(100 \%)$ \\
\hline
\end{tabular}

Tabelle 8 ist aufgrund der geringen Anzahl der Patienten mit PCI und Linksversorgungstyp nur eingeschränkt zu beurteilen. Es wird aber der Einfluss eines akuten Infarktes des RCX auf die Prognose bei STEMI-Patienten mit CTO $_{\text {NIRA }}$ und Linksversorgungstyp deutlich. Von den insgesamt sieben Patienten mit akutem RCX-Verschluss verstarben drei Patienten (42,9\%) noch im Krankenhaus.

Tabelle 8: Zusammenhang zwischen CTO- und Infarktgefäß mit Blick auf das Patientenoutcome (STEMI-Patienten mit $1 \mathrm{CTO}_{\text {NIRA }}$, Linksversorgungstyp und PCI $\mathrm{n}=9$ )

\begin{tabular}{|l|c|c|c|c|c|c|}
\hline CTO $_{\text {NIRA-Gefäß }}$ & \multicolumn{2}{|c|}{ RIVA (n= 1) } & \multicolumn{2}{c|}{ RCX (n = 0) } & \multicolumn{2}{c|}{ RCA (n= 8) } \\
\hline Infarktgefäß & RCA & RCX & RIVA & RCA & RIVA & RCX \\
\hline Anzahl & 0 & $1(100 \%)$ & - & - & $2(25 \%)$ & $6(75 \%)$ \\
\hline Shock & - & $1(100 \%)$ & - & - & $1(50 \%)$ & $1(17 \%)$ \\
\hline Tod KHS & - & $1(100 \%)$ & - & - & 0 & $2(33 \%)$ \\
\hline Tod 30-Tage & - & $1(100 \%)$ & - & - & 0 & $2(33 \%)$ \\
\hline
\end{tabular}


4.3.5.4 Kurzzeitsterblichkeit und TIMI Risk Score abhängig vom CTO-Gefäßabschnitt

In der Zusammenschau aller drei großen Koronargefäße stellte sich heraus, dass die höchste Krankenhaussterblichkeit bei STEMI-Patienten mit einem chronischen Verschluss in den proximalen Gefäßabschnitten zu finden war (Abbildung 38). Von den 53 Patienten mit $\mathrm{CTO}_{\text {NIRA }}$ proximal verstarben $32,1 \%(\mathrm{n}=17)$. In den übrigen Gefäßabschnitten (medial, distal und peripher) zusammengefasst lag die Sterblichkeit bei $20 \%(\mathrm{n}=11 / 55$; $\mathrm{p}=0,152)$.

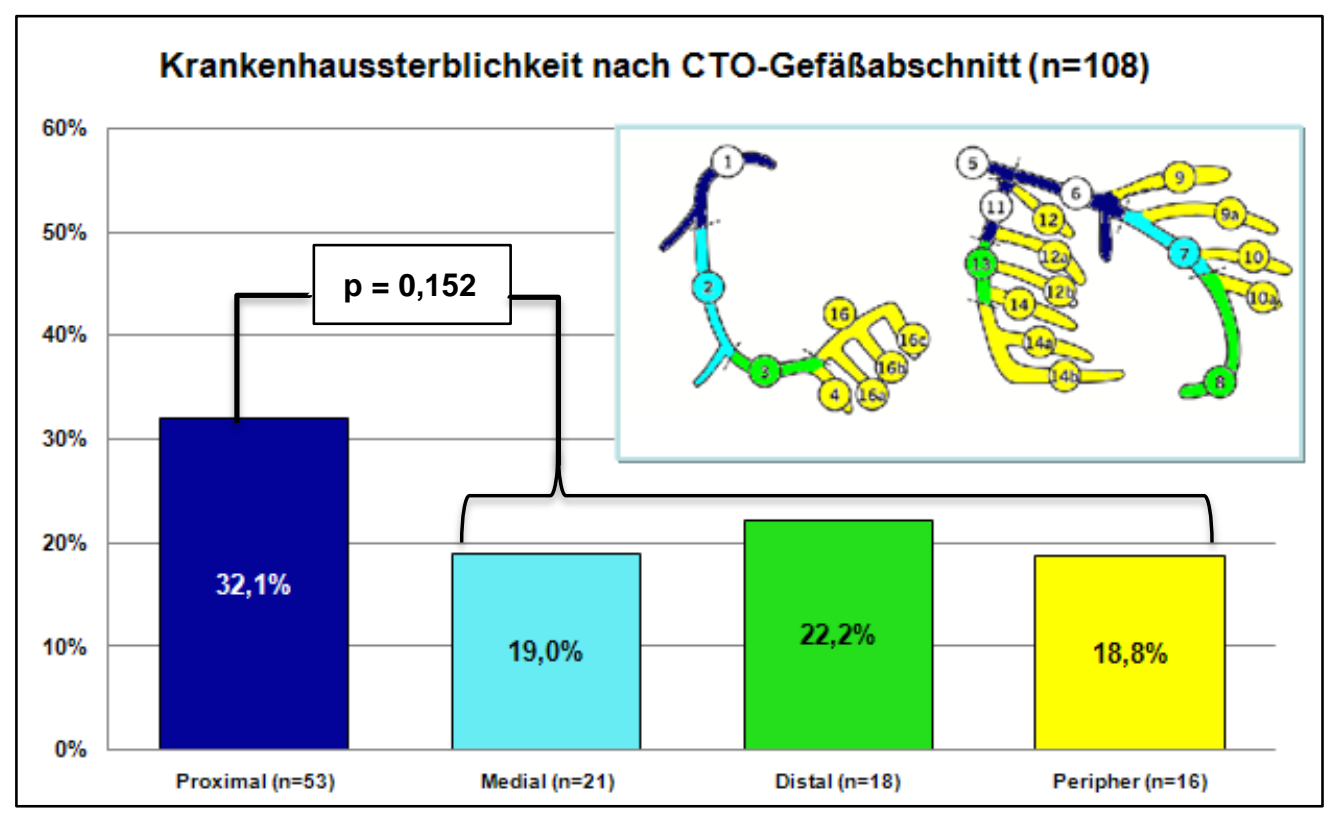

Abbildung 38: Krankenhaussterblichkeit (\%) in Abhängigkeit vom CTO-Gefäßabschnitt

Darüber hinaus wurde auch in diesen Untergruppen der TIMI Risk Score bestimmt. Das höchste Risikoprofil nach dem TIMI Risk Score hatten demnach Patienten mit einem $\mathrm{CTO}_{\text {NIRA }}$ in einem medialen Gefäßabschnitt (TRS: 5,43 Punkte), gefolgt von der Gruppe $\mathrm{CTO}_{\text {NIRA }}$ in einem peripheren Abschnitt (TRS: 5,13 Punkte) sowie der Gruppe CTO $\mathrm{NIRA}_{\text {im }}$ proximalen Abschnitt (TRS: 4,64 Punkte) und der Gruppe CTO $_{\text {NIRA }}$ im distalen Abschnitt (TRS: 4,61 Punkte).

Des Weiteren war auch der Anteil der Patienten im kardiogenen Schock in der Gruppe mit $\mathrm{CTO}_{\mathrm{NIRA}}$ in einem proximalen Abschnitt mit $40 \%$ vs. $24 \%$ in den übrigen Abschnitten am höchsten (p-Wert: 0,074; Abbildung 39). Die LVEF war zudem bei STEMI-Patienten mit proximalem $\mathrm{CTO}_{\mathrm{NIRA}}$ mit durchschnittlich $41,4 \%$ tendenziell geringer als in den übrigen Abschnitten zusammengefasst mit durchschnittlich 42,9\%. 


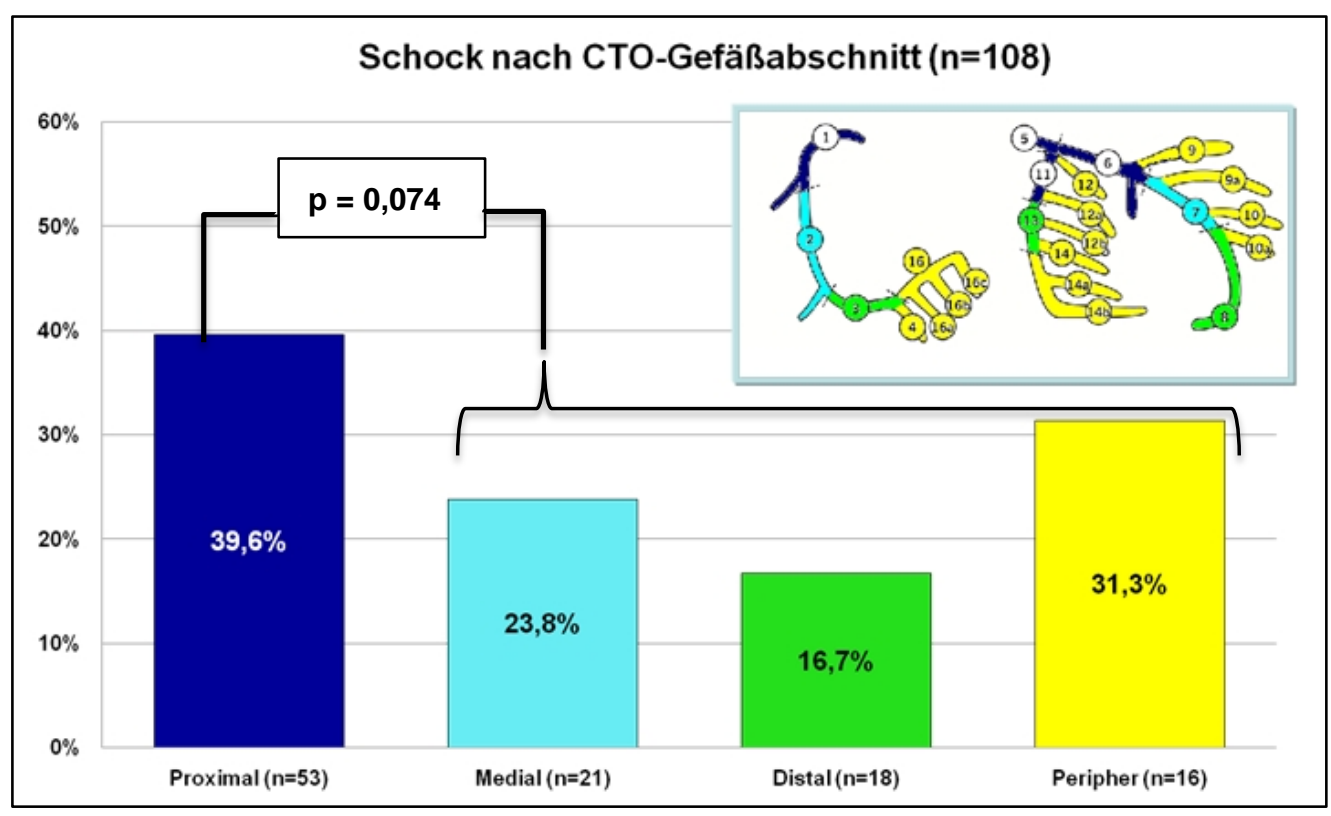

Abbildung 39: Anteil der Patienten mit Schock (\%) in Abhängigkeit vom CTOGefäßabschnitt

\subsubsection{Kurzzeitsterblichkeit abhängig von der CTO-Segmentlokalisation und dem Koronarversorgungstyp}

In den folgenden Abbildungen wird jeweils der Einfluss der Segmentlokalisation des $\mathrm{CTO}_{\text {NIRA }}$ in den einzelnen Koronargefäßen (siehe Kapitel 3.5: Abbildung 1) auf die Krankenhaussterblichkeit dargestellt. Dabei wurde in den Diagrammen eine Unterteilung in Rechts- und Linksversorgungstyp unternommen, um die Bedeutung des Koronarversorgungstyps für die Prognose dieser Patienten zu berücksichtigen.

Insgesamt befand sich bei 52 Patienten mit MVD $+1 \mathrm{CTO}_{\mathrm{NIRA}}$ ein chronischer Verschluss in der RCA (siehe Kapitel 4.3.1: Abbildung 24 und 25), 42 von diesen Patienten (81 \%) hatten einen Rechtsversorgungstyp und zehn Patienten (19\%) wiesen einen Linksversorgungstyp auf. Von den 42 Patienten mit $\mathrm{CTO}_{\mathrm{NIRA}}$ der RCA und Rechtsversorgungstyp verstarben im Krankenhaus 14 Patienten (33,3 \%). In der Gruppe der Patienten mit $\mathrm{CTO}_{\text {NIRA }}$ der RCA und Linksversorgungstyp lag die Krankenhaussterblichkeit bei $20 \%$ $(\mathrm{n}=2)$. Abbildung 40 präsentiert darüber hinaus die Krankenhaussterblichkeit in Abhängigkeit von der jeweiligen CTO-Segmentlokalisation in der RCA. Hier konnte bei Patienten mit einem $\mathrm{CTO}_{\mathrm{NIRA}}$ im proximalen RCA-Segment 1 bei Rechtsversorgungstyp eine außerordentlich hohe Krankenhaussterblichkeit ausgemacht werden. Von den 26 Patienten in dieser Gruppe verstarben im Krankenhaus elf Patienten (42,3 \%), die 30-Tage Sterblichkeit lag bei 46,2\% ( $\mathrm{n}=12$ Patienten). Von den sechs Patienten mit Linksversorgungstyp und $\mathrm{CTO}_{\mathrm{NIRA}}$ in Segment 1 der RCA verstarb ein Patient (16,7 \%). 


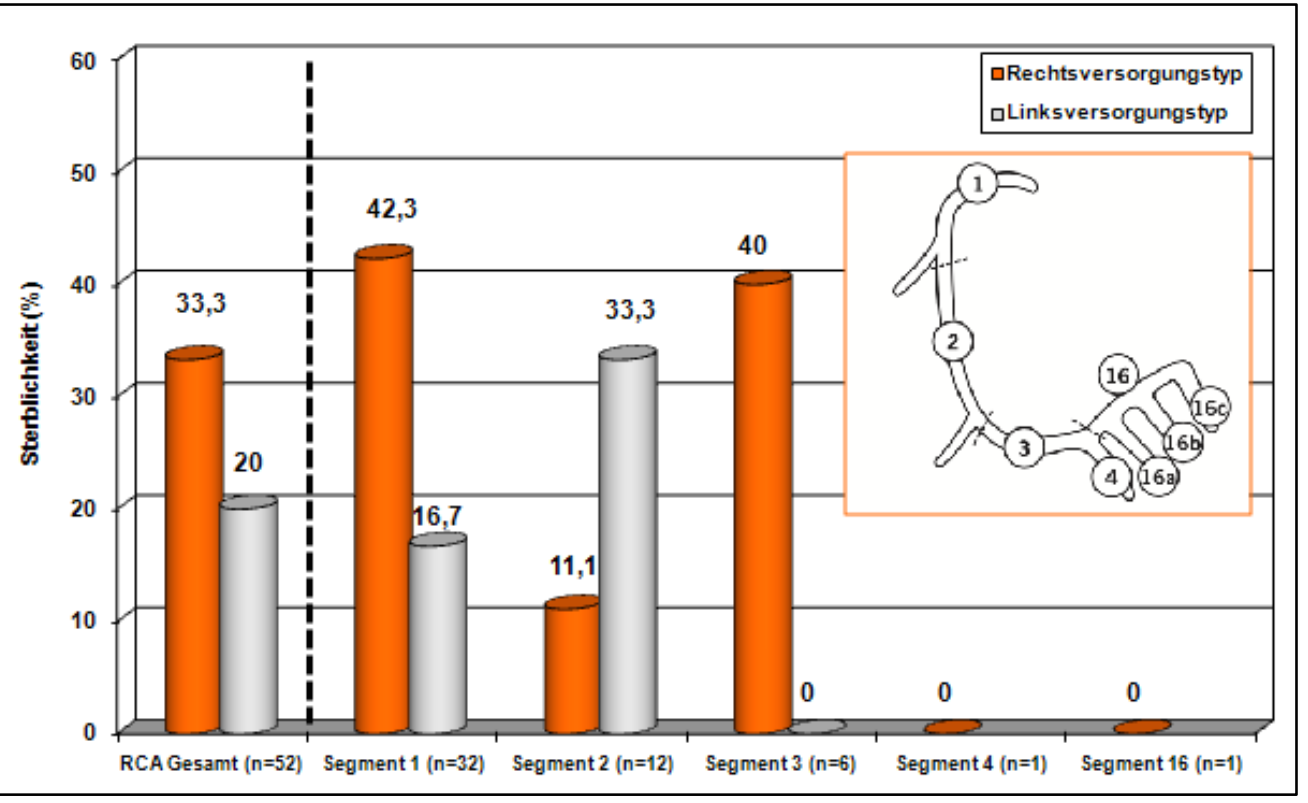

Abbildung 40: Krankenhaussterblichkeit (\%) nach CTO-Gefäßsegmenten in der RCA in Abhängigkeit vom Versorgungstyp

STEMI-Patienten mit einem CTO $_{\text {NIRA }}$ in Segment 1 der RCA bei Rechtsversorgungstyp scheinen eine besondere Risikogruppe zu bilden. Im Vergleich zu allen anderen STEMIPatienten mit $\mathrm{CTO}_{\text {NIRA }}$ zusammengefasst $(\mathrm{n}=82)$ war der Unterschied sowohl in der Krankenhaussterblichkeit (42,3\% vs. 20,7 \%) als auch in der 30-Tage-Sterblichkeit (46,2\% vs. $20,7 \%$ ) statistisch signifikant (p-Wert $\operatorname{Tod}_{\mathrm{KHS}}: 0,029$; $\mathrm{p}$-Wert $\left.\operatorname{Tod}_{30 \mathrm{~d}}: 0,011\right)$. Zudem ergab sich zwischen diesen beiden Gruppen im Durchschnittsalter (60,9 Jahre vs. 67,9 Jahre; $\mathrm{p}=0,009)$ sowie in der Lokalisation des Akut-Infarktgefäßes $(\mathrm{p}=0,005)$ ein statistisch signifikanter Unterschied (siehe Anhang, Kapitel 7.4, Tabelle 18). Auch der Anteil an Patienten im kardiogenen Schock lag in der Gruppe der STEMI-Patienten mit $\mathrm{CTO}_{\text {NIRA }}$ in Segment 1 der RCA und Rechtsversorgungstyp deutlich, aber statistisch nicht signifikant, höher als in der Vergleichsgruppe (46\% vs. $27 \%$; = 0,06). Der TIMI Risk Score war dagegen in der Gruppe der Patienten mit CTO $_{\text {NIRA }}$ in Segment 1 der RCA und Rechtsversorgungstyp mit 4,69 Punkten niedriger als in der Gruppe aller anderen CTO $_{\text {NIRA }}{ }^{-}$ Patienten mit 4,91 Punkten.

In einer zunächst univariaten Analyse wurden im Rahmen eines Vortests die STEMIPatienten mit $\mathrm{CTO}_{\text {NIRA }}$ in die Gruppen mit und ohne Tod im Krankenhaus eingeteilt. Unter den verstorbenen Patienten befanden sich dabei signifikant häufiger Patienten mit der CTO-Lokalisation „RCA-Segment 1 bei Rechtsversorgungstyp“ als unter den nicht verstorbenen Patienten (39 \% vs. $19 \%$; $=0$,029). Zudem konnten weitere relevante Unterschiede (hier p-Wert <0,1) zwischen den Gruppen mit und ohne Tod im Krankenhaus bei folgenden Merkmalen ermittelt werden: Hinterwandinfarkt, Patientenalter, positive Familienanamnese, Anteil Raucher/in, Z. n. PCI, bekannte Nieren- 
insuffizienz, kardiogener Schock, 3-Gefäß-KHK und Kollateralversorgung. All diese Variablen wurden in ein multivariates Logit-Modell (logistische Regression) zur Identifizierung potentiell unabhängiger Prädiktoren der Krankenhaussterblichkeit bei STEMIPatienten mit $\mathrm{CTO}_{\mathrm{NIRA}}$ eingespeist (zuzüglich bekannter, aber hier nicht signifikanter Prädiktoren wie das Geschlecht sowie ein Vorderwandinfarkt im EKG). Zudem wurde der Versorgungstyp in dieser Analyse berücksichtigt, da definitionsbedingt in der Gruppe mit CTO-Lokalisation „RCA-Segment 1 bei Rechtsversorgungstyp“ kein Patient mit Linksversorgungstyp vorkommt. Gegebenenfalls hatte dies aber einen besonderen Einfluss auf die Prognose. Tabelle 9 präsentiert das endgültige Modell dieser multivariaten Analyse. Hier konnte gezeigt werden, dass in der vorliegenden Untersuchung unter Berücksichtigung der genannten relevanten Variablen bei STEMI-Patienten mit $\mathrm{CTO}_{\text {NIRA }}$ ein chronischer Verschluss in Segment 1 der RCA bei Rechtsversorgungstyp ein unabhängiger Prädiktor der Krankenhaussterblichkeit war (OR 6,01; 95 \% CI: 1,08 - 33,53; p = 0,041).

Tabelle 9: Krankenhaussterblichkeit und unabhängige Prädiktoren bei STEMI-Patienten mit $1 \mathrm{CTO}_{\text {NIRA }}(\mathrm{n}=108)$; Multivariates Logit-Modell nach Vortest

\begin{tabular}{|c|c|c|c|c|c|c|c|}
\hline \multirow[b]{2}{*}{ Prädiktor } & \multicolumn{4}{|c|}{ Univariate Analyse (Vortest) } & \multicolumn{3}{|c|}{ Multivariate Analyse } \\
\hline & & $\begin{array}{c}\text { Tod KHS ja } \\
(n=28)\end{array}$ & $\begin{array}{c}\text { Tod KHS } \\
\text { nein }(n=80)\end{array}$ & p-Wert & $\begin{array}{l}\text { Odds } \\
\text { Ratio }\end{array}$ & $95 \% \mathrm{CI}$ & p-Wert \\
\hline \multirow{4}{*}{ EKG (Infarktlokalisation) } & VWI & $14(50 \%)$ & $33(41 \%)$ & 0,422 & 1,35 & $0,2-9,02$ & 0,755 \\
\hline & HWI & $7(25 \%)$ & $37(46 \%)$ & 0,049 & 0,56 & $0,07-4,26$ & 0,578 \\
\hline & LWI & $5(18 \%)$ & $8(10 \%)$ & 0,271 & & & \\
\hline & LSB & $2(7 \%)$ & $2(3 \%)$ & 0,263 & & & \\
\hline Frauen & & $5(18 \%)$ & $15(19 \%)$ & \multirow{2}{*}{0,917} & \multirow{2}{*}{0,31} & \multirow{2}{*}{$0,05-2,0$} & \multirow{2}{*}{0,218} \\
\hline Männer & & $23(82 \%)$ & $65(81 \%)$ & & & & \\
\hline Alter \pm Standardabweichung & Mittelwert & $70,2 \pm 9,22$ & $64,9 \pm 12,4$ & 0,041 & 1,06 & $0,98-1,14$ & 0,136 \\
\hline BMI $\left(\mathrm{Kg} / \mathrm{m}^{2}\right)$ & Mittelwert & $27,8 \pm 3,43$ & $27,8 \pm 3,62$ & 0,983 & & & \\
\hline Arterielle Hypertonie & & $19(68 \%)$ & $46(58 \%)$ & 0,335 & & & \\
\hline Diabetes mellitus & & $12(43 \%)$ & $24(30 \%)$ & 0,214 & & & \\
\hline Frühere Angina Pectoris & & $7(25 \%)$ & $22(28 \%)$ & 0,797 & & & \\
\hline Hyperlipidämie & & $8(29 \%)$ & $25(31 \%)$ & 0,791 & & & \\
\hline Positive Familienanamnese & & $1(4 \%)$ & $16(20 \%)$ & 0,04 & 0,82 & $0,06-12,0$ & 0,883 \\
\hline Raucher/in & & $5(18 \%)$ & $38(48 \%)$ & 0,006 & 0,19 & $0,03-1,18$ & 0,075 \\
\hline Anamnestisch Myokardinfarkt & & $6(21 \%)$ & $12(15 \%)$ & 0,432 & & & \\
\hline Anamnestisch Apoplex & & $4(14 \%)$ & $8(10 \%)$ & 0,535 & & & \\
\hline Vorherige PCI & & $7(25 \%)$ & $7(9 \%)$ & 0,028 & 10,59 & $1,34-83,87$ & 0,025 \\
\hline Bekannte Niereninsuffizienz & & $7(25 \%)$ & $7(9 \%)$ & 0,028 & 2 & $0,27-14,67$ & 0,497 \\
\hline Präklinische Reanimation & & $7(25 \%)$ & $10(13 \%)$ & 0,118 & & & \\
\hline Kardiogener Schock & & $21(75 \%)$ & $13(16 \%)$ & $<0,001$ & 35,92 & $7,03-183,43$ & $<0,001$ \\
\hline \multicolumn{5}{|l|}{ Angiographische Befunde: } & & & \\
\hline 3-Gefäß-KHK (inkl. Hauptstammstenose) & & $26(93 \%)$ & $61(76 \%)$ & 0,056 & 2,66 & $0,27-26,69$ & 0,404 \\
\hline $\begin{array}{l}\text { CTO in Segment } 1 \text { der RCA bei } \\
\text { Rechtsversorgungstyp }\end{array}$ & & $11(39 \%)$ & $15(19 \%)$ & 0,029 & 6,01 & $1,08-33,53$ & 0,041 \\
\hline \multirow{2}{*}{ Versorgungstyp } & Rechts & $25(89 \%)$ & $72(90 \%)$ & \multirow{2}{*}{0,914} & \multirow{2}{*}{4,93} & \multirow{2}{*}{$0,39-61,83$} & \multirow{2}{*}{0,216} \\
\hline & Links & $3(11 \%)$ & $8(10 \%)$ & & & & \\
\hline Kollateralen & & $21(75 \%)$ & $71(89 \%)$ & 0,078 & 0,43 & $0,05-3,6$ & 0,44 \\
\hline PCI durchgeführt & & $22(79 \%)$ & $65(81 \%)$ & 0,758 & & & \\
\hline
\end{tabular}

Dieses Ergebnis konnte in einer rückwärtsgerichteten schrittweisen Berechnung des LogitModells (backward stepwise) unter Berücksichtigung weiterer Variablen (siehe Anhang, Kapitel 7.4, Tabelle 19) bestätigt werden. Im endgültigen Modell waren dabei lediglich die Variablen Alter, Schock, Raucher/in und Z. n. PCI sowie ein CTO $_{\text {NIRA }}$ in Segment 1 der 
RCA bei Rechtsversorgungstyp (OR 5,08; $95 \%$ CI: 1,15 - 22,54; p = 0,032) signifikante Prädiktoren der Krankenhaussterblichkeit in der Gesamtgruppe aller STEMI-Patienten mit $\mathrm{CTO}_{\text {NIRA }}($ Tabelle 10).

Tabelle 10: Unabhängige Prädiktoren der Krankenhaussterblichkeit bei STEMI-Patienten mit $1 \mathrm{CTO}_{\text {NIRA }}(\mathrm{n}=108)$; Multivariates Logit-Modell nach backward stepwise-Methode

\begin{tabular}{|l|c|c|c|}
\hline & \multicolumn{3}{|c|}{ Multivariat backward stepwise } \\
\hline Prädiktor & Odds Ratio & $\mathbf{9 5} \% \mathbf{C I}$ & $\mathbf{p}$-Wert \\
\hline Alter & 1,07 & $1,0-1,15$ & $\mathbf{0 , 0 4 5}$ \\
\hline Raucher/in & 0,21 & $0,05-0,94$ & $\mathbf{0 , 0 4 2}$ \\
\hline Vorherige PCI & 9,93 & $1,63-60,43$ & $\mathbf{0 , 0 1 3}$ \\
\hline Kardiogener Schock & 33,15 & $7,5-146,41$ & $<\mathbf{0 , 0 0 1}$ \\
\hline CTO RCA Segment 1 mit Rechtsversorgung & 5,08 & $1,15-22,54$ & $\mathbf{0 , 0 3 2}$ \\
\hline
\end{tabular}

Tabelle 11 zeigt darüber hinaus die Prognose der Patienten mit CTO $_{\text {NIRA }}$ in Segment 1 der RCA bei Rechtsversorgungstyp aufgeteilt nach dem jeweiligen akuten Infarktgefäß. Es ist $\mathrm{zu}$ beachten, dass in dieser Tabelle lediglich die Patienten mit erfolgter primärer PCI berücksichtigt wurden, dabei konnte kein signifikanter Unterschied zwischen dem RIVA und dem RCX als akutes Infarktgefäß festgestellt werden.

Tabelle 11: Zusammenhang zwischen CTO- und Infarktgefäß mit Blick auf das Patienten-

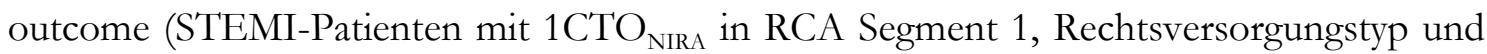
PCI $; \mathrm{n}=18)$

\begin{tabular}{|l|c|c|c|c|}
\hline CTO $_{\text {NIRA-Gefäß }}$ & \multicolumn{4}{|c|}{ Proximale RCA mit Rechtsversorgungstyp und PCI (n = 18) } \\
\hline Infarktgefäß & RIVA & RCX & LMCA & $\begin{array}{c}\text { p-Wert } \\
\text { (RIVA vs. RCX) }\end{array}$ \\
\hline Anzahl & 11 & 6 & 1 & 0,858 \\
\hline Shock & $5(45 \%)$ & $3(50 \%)$ & $1(100 \%)$ & 0,901 \\
\hline Tod KHS & $4(36 \%)$ & $2(33 \%)$ & $1(100 \%)$ & 0,627 \\
\hline Tod 30-Tage & $5(45 \%)$ & $2(33 \%)$ & $1(100 \%)$ & \\
\hline
\end{tabular}

In der Gruppe der Patienten mit MVD +1CTO ${ }_{\text {NIRA }}$ im RIVA wurden 23 Patienten erfasst. Fast alle Patienten zeigten koronarangiographisch einen Rechtsversorgungstyp (96\%, $\mathrm{n}=22$ ), lediglich ein Patient (4\%) wies einen Linksversorgertyp auf. Dieser Patient mit Linksversorgertyp hatte einen $\mathrm{CTO}_{\text {NIRA }}$ in Segment 7 und verstarb im Krankenhaus. Bei den Patienten mit Rechtsversorgung und $\mathrm{CTO}_{\text {NIRA }}$ im RIVA lag die Krankenhaussterblichkeit bei 18,2 \% ( $\mathrm{n}=4)$. Die höchste Krankenhaussterblichkeit bei CTO $_{\text {NIRA }}$ im Versorgungsbereich des RIVA präsentierte sich bei den Patienten mit Rechts- 
versorgungstyp und $\mathrm{CTO}_{\mathrm{NIRA}}$ im proximalen Segment 6. Von den zehn Patienten dieser Gruppe verstarben drei (30\%; Abbildung 41).

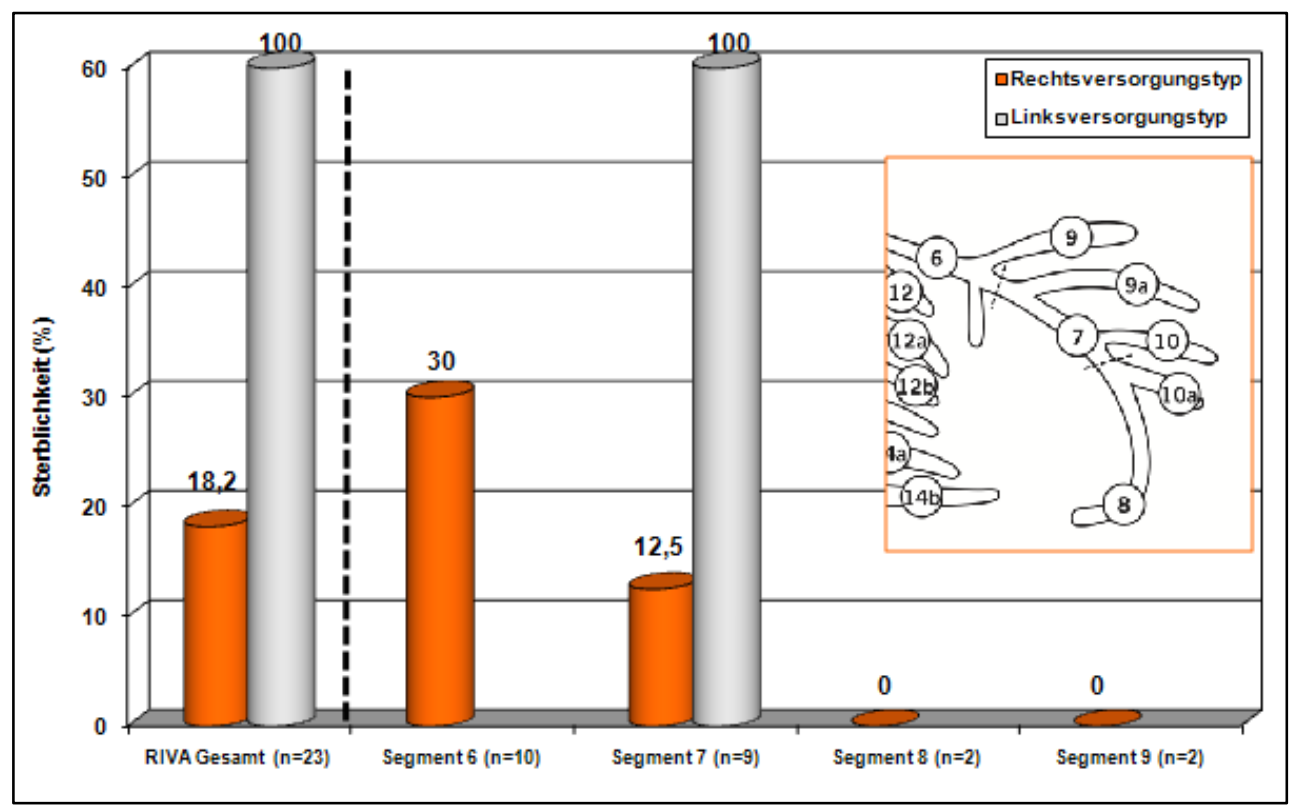

Abbildung 41: Krankenhaussterblichkeit (\%) nach CTO-Gefäßsegmenten im RIVA in Abhängigkeit vom Versorgungstyp

Die Krankenhaussterblichkeit aller Patienten mit MVD $+1 \mathrm{CTO}_{\mathrm{NIRA}}$ im RCX ( $\mathrm{n}=33$ ) betrug 21,2\% ( $n=7)$. Bei allen Patienten dieser Gruppe wurde angiographisch ein Rechtsversorgungstyp diagnostiziert. Die Sterblichkeit bei Patienten mit CTO $_{\text {NIRA }}$ in den Segmenten 11, 13 und 14 lag zwischen 18,2 \% - 20,0 \% und war damit relativ konstant. Segment 14a zeigt einen Ausreißer (Krankenhaussterblichkeit: 50 \%), dieser hohe Wert ist aufgrund der äußerst geringen Patientenzahl $(\mathrm{n}=2)$ nicht aussagekräftig (Abbildung 42).

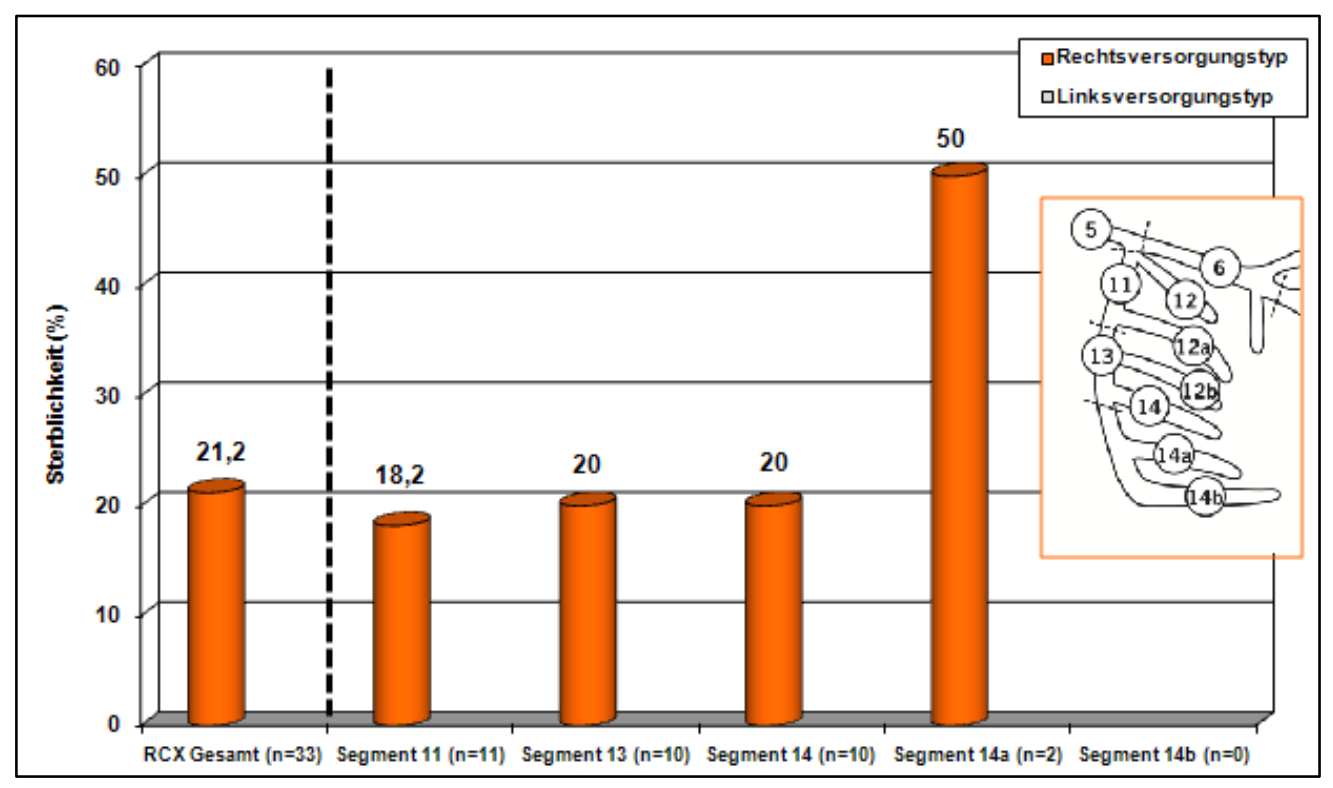

Abbildung 42: Krankenhaussterblichkeit (\%) nach CTO-Gefäßsegmenten im RCX in Abhängigkeit vom Versorgungstyp 


\subsubsection{Kurzzeitsterblichkeit abhängig von der Kollateralversorgung}

In Abbildung 43 wird die Krankenhaussterblichkeit aller Patienten mit MVD +1CTO NIRA ( $\mathrm{n}=108)$ in Abhängigkeit von einer möglichen Kollateralversorgung dargestellt. In der Gruppe der Patienten mit angiographisch sichtbaren Kollateralen $(\mathrm{n}=92)$ verstarben mit einer Tendenz zur Signifikanz $(p=0,078)$ deutlich weniger Patienten intrahospital $(22,8 \%$, $\mathrm{n}=21)$ als in der Gruppe ohne angiographisch sichtbare Kollateralversorgung (43,8 \%, $\mathrm{n}=7)$.

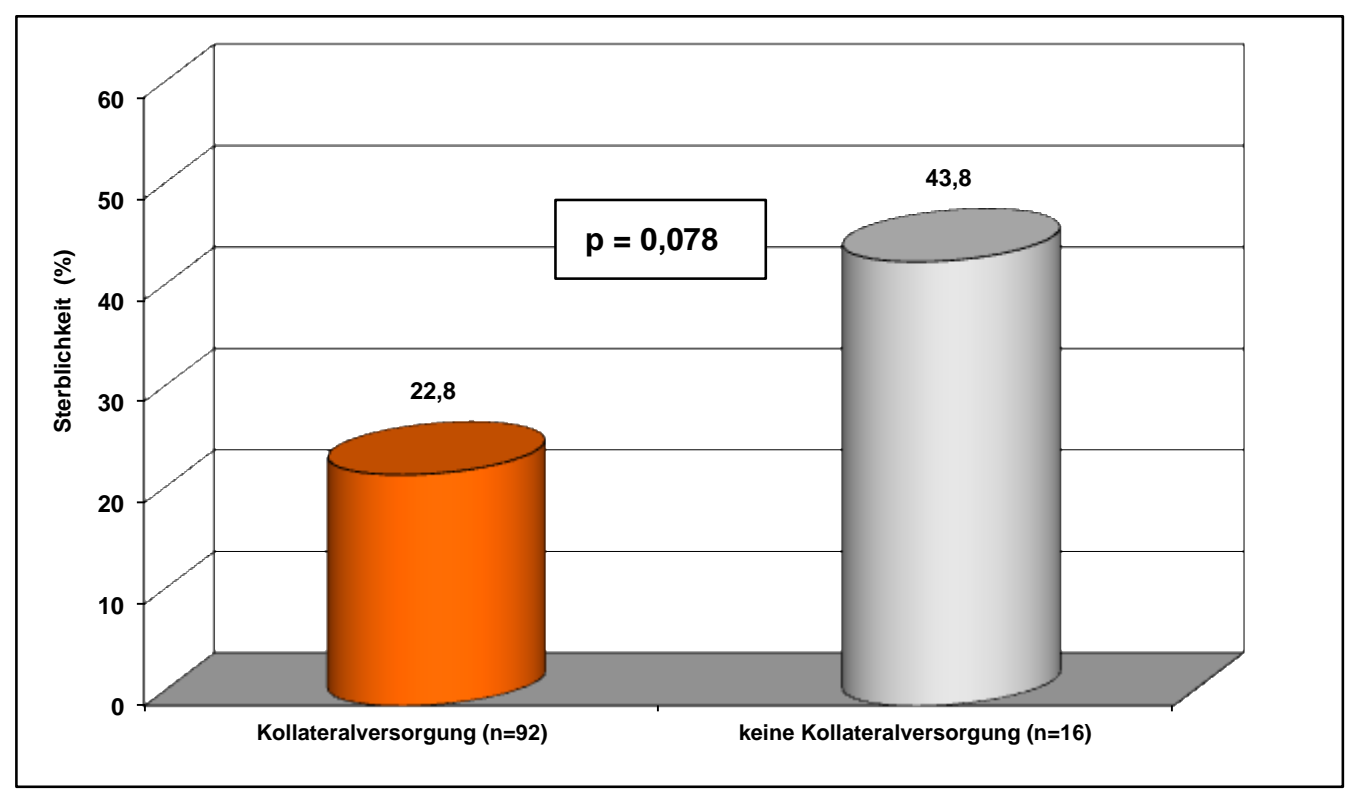

Abbildung 43: Krankenhaussterblichkeit (\%) in Abhängigkeit von der Kollateralversorgung (Alle Patienten mit $1 \mathrm{CTO}_{\mathrm{NIRA}}, \mathrm{n}=108$ )

Wie in Kapitel 4.3.3 dargelegt, wurde die Gruppe der 65 STEMI-Patienten mit MVD $+1 \mathrm{CTO}_{\mathrm{NIRA}}$, erfolgter primärer PCI und angiographisch sichtbarer interkoronarer Kollateralisierung weiter unterteilt. Hierbei zeigte sich jedoch lediglich ein minimaler, nicht signifikanter $(\mathrm{p}=0,947)$, Unterschied in der Krankenhaussterblichkeit zwischen solchen Patienten, die eine Kollateralversorgung über das vom akuten Infarkt betroffene Gefäß $(21,2 \% ; n=33)$ erhielten und solchen Patienten, die eine Kollateralversorgung über ein nicht vom Infarkt betroffenes Gefäß erhielten (21,9\%; $\mathrm{n}=32$; Abbildung 44). 


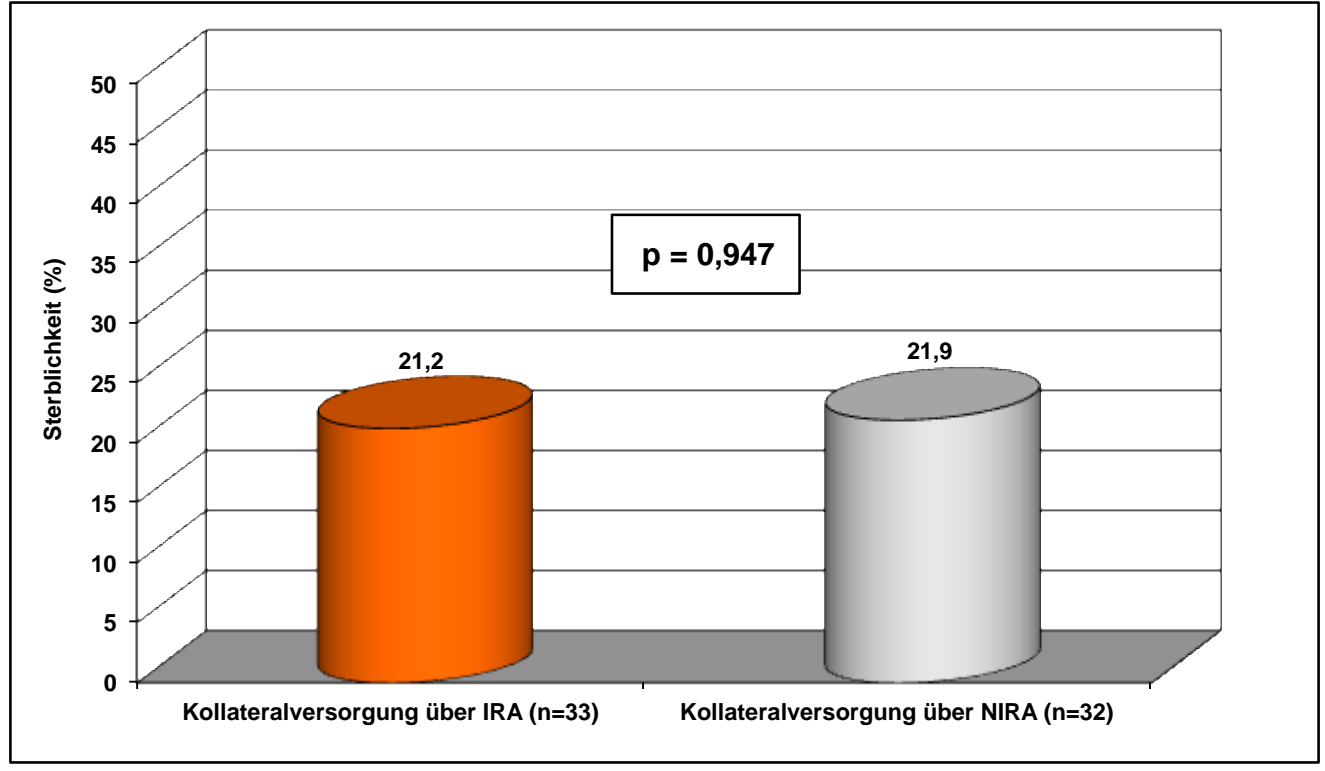

Abbildung 44: Krankenhaussterblichkeit (\%) nach Herkunft der Kollaterale (Alle PCIPatienten mit $1 \mathrm{CTO}_{\mathrm{NIRA}}$ und interkoronarer Kollateralversorgung, $\mathrm{n}=65$ )

Darüber hinaus wurde die Gruppe der 33 PCI-Patienten mit angiographisch sichtbarer Kollateralversorgung des chronisch verschlossenen Areals über das vom akuten Infarkt betroffene Gefäß genauer analysiert (siehe Kapitel 4.3.3: Abbildung 33). Es zeigte sich dabei ein deutlicher Unterschied in der Krankenhaussterblichkeit zwischen der Patientengruppe mit Kollateralursprung proximal $(n=18)$ und der Gruppe mit Kollateralursprung distal des akuten Verschlusses $(\mathrm{n}=15)$. Mit einer Krankenhaussterblichkeit von 33,3 \% in der erstgenannten Gruppe $(\mathrm{n}=6)$ und einer Sterblichkeit von 6,7\% in der Letzteren $(\mathrm{n}=1)$ war hierbei eine statistische Tendenz zu erkennen ( $p=0,062$; Abbildung 45).

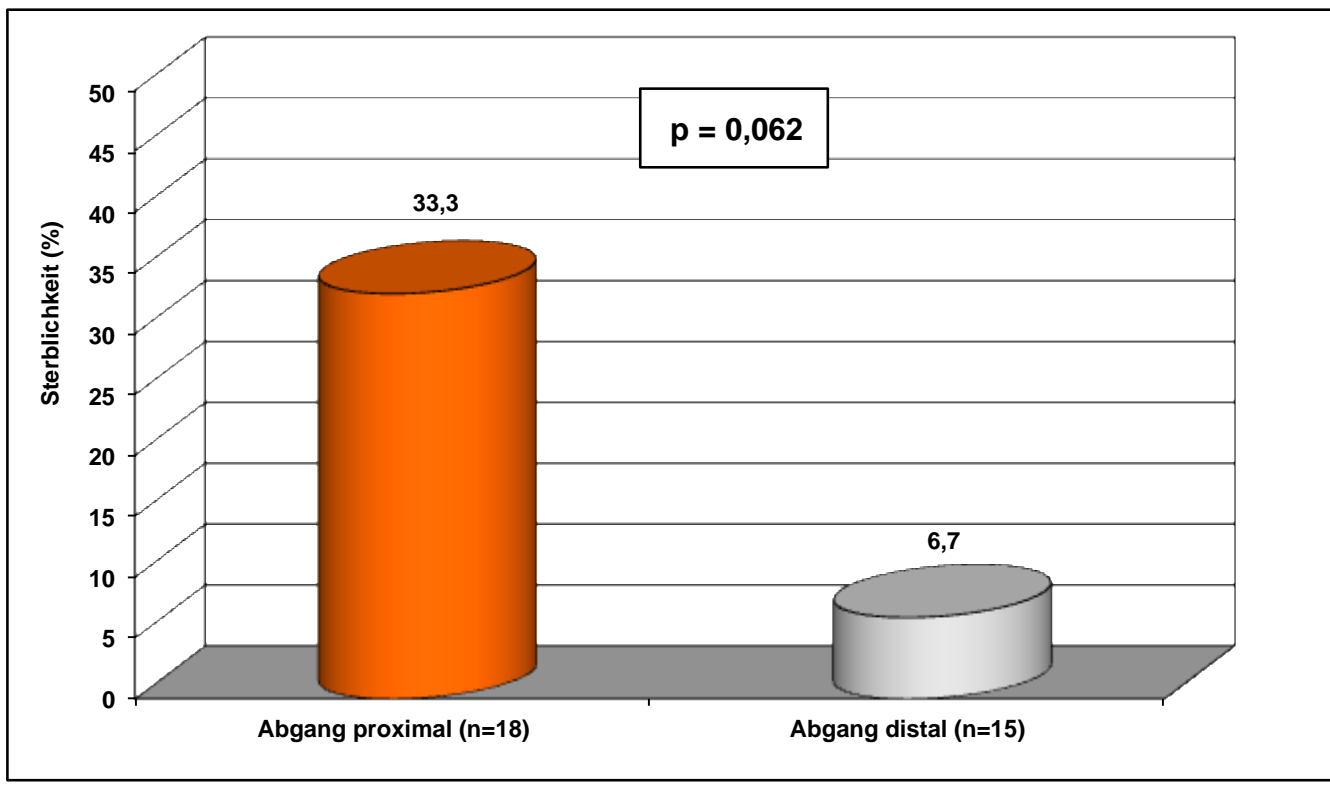

Abbildung 45: Krankenhaussterblichkeit (\%) nach Ursprungsort der IRA-Kollaterale (Alle PCI-Patienten mit $1 \mathrm{CTO}_{\text {NIRA }}$ und Kollateralversorgung über IRA, $\mathrm{n}=33$ ) 
Die folgenden Tabellen 12, 13 und 14 präsentieren zudem das Patientenoutcome abhängig von der Kollateralisierung und dem Kollateralursprung in Bezug auf das jeweilige $\mathrm{CTO}_{\text {NIRA }^{-}}$ Gefäß bei allen STEMI-Patienten mit $1 \mathrm{CTO}_{\text {NIRA }}$. Aufgrund der relativ geringen Patientenzahlen in den restlichen Untergruppen waren hierbei vor allem die Ergebnisse der Patienten mit $\mathrm{CTO}_{\mathrm{NIRA}}$ in der RCA von Bedeutung. Es zeigte sich, dass selbst bei einer vorhandenen Kollateralversorgung die Krankenhaussterblichkeit der Patienten mit $\mathrm{CTO}_{\text {NIRA }}$ in der RCA bei $28 \%$ lag. Am häufigsten lag bei diesen Patienten der Kollateralursprung im RIVA (52\% aller Patienten mit CTO $_{\text {NIRA }}$ in der RCA), von diesen Patienten verstarben $26 \%$ im Krankenhaus.

Tabelle 12: Kollateralisierung bei STEMI-Patienten mit $1 \mathrm{CTO}_{\text {NIRA }}$ in der RCA ( $\left.\mathrm{n}=52\right)$

\begin{tabular}{|l|c|c|}
\hline & Prävalenz (\%) & Tod KHS (\%) \\
\hline Kollateralisierung & $90(\mathrm{n}=47)$ & 27,7 \\
\hline Keine Kollateralisierung & $10(\mathrm{n}=5)$ & 60 \\
\hline Kollateralursprung: & & \\
\hline - von RIVA & $52(\mathrm{n}=27)$ & 25,9 \\
\hline - von RCX & $46(\mathrm{n}=24)$ & 25,0 \\
\hline - über Brücken/intrakoronar & $19(\mathrm{n}=10)$ & 20,0 \\
\hline - von IRA (Pat. mit PCI) & $46(\mathrm{n}=18 / 39)$ & 27,8 \\
\hline
\end{tabular}

Tabelle 13: Kollateralisierung bei STEMI-Patienten mit $1 \mathrm{CTO}_{\text {NIRA }}$ im RIVA $(\mathrm{n}=23)$

\begin{tabular}{|l|c|c|}
\hline & Prävalenz (\%) & Tod KHS (\%) \\
\hline Kollateralisierung & $87(\mathrm{n}=20)$ & 25 \\
\hline Keine Kollateralisierung & $13(\mathrm{n}=3)$ & 0 \\
\hline Kollateralursprung: & & \\
\hline - von RCX & $26(\mathrm{n}=6)$ & 33,3 \\
\hline - von RCA & $52(\mathrm{n}=12)$ & 16,7 \\
\hline - über Brücken/intrakoronar & $30(\mathrm{n}=7)$ & 28,6 \\
\hline - von IRA (Pat. mit PCI) & $52(\mathrm{n}=11 / 21)$ & 18,2 \\
\hline
\end{tabular}

Tabelle 14: Kollateralisierung bei STEMI-Patienten mit 1 CTO $_{\text {NIRA }}$ im RCX ( $\mathrm{n}=33$ )

\begin{tabular}{|l|c|c|}
\hline & Prävalenz (\%) & Tod KHS (\%) \\
\hline Kollateralisierung & $76(\mathrm{n}=25)$ & 12 \\
\hline Keine Kollateralisierung & $24(\mathrm{n}=8)$ & 50 \\
\hline Kollateralursprung: & & \\
\hline - von RIVA & $33(\mathrm{n}=11)$ & 9,1 \\
\hline - von RCA & $39(\mathrm{n}=13)$ & 7,7 \\
\hline - über Brücken/intrakoronar & $21(\mathrm{n}=7)$ & 14,3 \\
\hline - von IRA (Pat. mit PCI) & $15(\mathrm{n}=4 / 27)$ & 0 \\
\hline
\end{tabular}




\subsubsection{Kurzzeitsterblichkeit bei Patienten mit und ohne Hinweis auf einen früheren} Myokardinfarkt

In Kapitel 4.3.4 wurde dargestellt, dass von den 108 Patienten mit MVD +1CTO NIRA $_{44} 44$ Patienten anamnestisch bzw. elektrokardiographisch einen Indikator für einen alten bzw. abgelaufenen Myokardinfarkt aufwiesen. Während die Krankenhaussterblichkeit bei Patienten ohne Hinweis auf einen stattgehabten Infarkt bei 21,9\% lag, verstarben von den Patienten mit Hinweis auf einen alten Infarkt 31,8 \% ( $p=0,247)$. Tabelle 15 demonstriert diesen deutlichen Unterschied in der Krankenhaussterblichkeit und präsentiert zudem die Ergebnisse in Bezug auf die einzelnen vom CTO betroffenen Koronargefäße.

Auffällig ist hierbei die Ausnahmestellung bei chronischen Verschlüssen der RCA. Während in den Gruppen mit CTO $_{\text {NIRA }}$ im RCX und im RIVA die Krankenhaussterblichkeit bei Patienten mit einem Hinweis auf einen alten Infarkt deutlich höher war als bei Patienten ohne Hinweis auf einen alten Infarkt (bei CTO-Lokalisation im RIVA mit $\mathrm{p}=0,015$ signifikant), war in der Patientengruppe mit CTO $_{\text {NIRA }}$ in der RCA die Krankenhaussterblichkeit bei Patienten ohne Hinweis auf einen alten Infarkt mit $37 \%$ deutlich, aber nicht signifikant höher als bei den Patienten mit Hinweis auf einen alten Infarkt mit $24 \%$.

Tabelle 15: Krankenhaussterblichkeit bei Patienten mit und ohne dokumentierten früheren Infarkt (Alle Patienten mit $1 \mathrm{CTO}_{\text {NIRA }}, \mathrm{n}=108$ )

\begin{tabular}{|c|c|c|c|c|}
\hline CTO-Gefäß & $\begin{array}{c}\text { Anzahl } \\
\text { Patienten }\end{array}$ & $\begin{array}{c}\text { Krankenhaussterblichkeit } \\
\text { bei Pat. mit Hinweis auf } \\
\text { alten Infarkt }\end{array}$ & $\begin{array}{c}\text { Krankenhaussterblichkeit } \\
\text { bei Pat. ohne Hinweis auf } \\
\text { alten Infarkt }\end{array}$ & p-Wert \\
\hline Gesamt & $\mathbf{1 0 8}$ & $\mathbf{1 4 / 4 4 ( 3 1 , 8 \% )}$ & $\mathbf{1 4 / 6 4 ( 2 1 , 9 \% )}$ & $\mathbf{0 , 2 4 7}$ \\
\hline RCA & $\mathbf{5 2}$ & $6 / 25(24,0 \%)$ & $10 / 27(37,0 \%)$ & $\mathbf{0 , 3 0 8}$ \\
\hline RIVA & $\mathbf{2 3}$ & $5 / 12(41,7 \%)$ & $0 / 11(0 \%)$ & $\mathbf{0 , 0 1 5}$ \\
\hline RCX & $\mathbf{3 3}$ & $3 / 7(42,9 \%)$ & $4 / 26(15,4 \%)$ & $\mathbf{0 , 1 1 5}$ \\
\hline
\end{tabular}




\section{Diskussion}

Der ST-Strecken-Hebungsinfarkt ist eine akut lebensbedrohliche Ausprägung der koronaren Herzkrankheit und zählt zu den führenden Todesursachen in Deutschland (Robert Koch-Institut 2015). Die Therapie zielt auf eine schnellstmögliche Wiedereröffnung des verschlossenen Koronargefäßes ab. Dabei gilt die perkutane Koronarintervention als Therapie der Wahl (Steg et al. 2012). Im Rahmen der Akut-Koronarangiographie kann anhand des Koronarstatus der Schweregrad der KHK festgelegt werden und eine Einschätzung des Risikos vorgenommen werden. Es hat sich gezeigt, dass Patienten mit einer Mehrgefäßerkrankung ein besonders hohes Sterblichkeitsrisiko aufweisen (Sorajja et al. 2007) und neuere Untersuchungen haben ergeben, dass hierbei dem chronischen Verschluss in einem Nicht-Infarkt-Gefäß $\left(\mathrm{CTO}_{\text {NIRA }}\right)$ eine besondere Bedeutung für die Sterblichkeit bei Mehrgefäßerkrankung zukommt (O'Connor et al. 2015). Bei STEMI-Patienten mit $\mathrm{CTO}_{\text {NIRA }}$ liegen allerdings bisher nur wenige Informationen zur Bedeutung angiographischer Befunde wie der CTO-Lokalisation, des Koronarversorgungstyps sowie des Kollateralstatus vor. Der Fokus der vorliegenden Arbeit lag daher auf einer detaillierten Analyse der Bedeutung des Koronarstatus bei STEMI-Patienten mit $\mathrm{CTO}_{\text {NIRA }}$. Die Untersuchung wurde an einem sehr großen Kollektiv von STEMI-Patienten durchgeführt, die prospektiv und unselektioniert im Rahmen des FITT-STEMI-QM-Projektes an der Göttinger Universitätsklinik erfasst worden waren.

Die Ergebnisse dieser Untersuchung und damit auch deren Interpretation und Diskussion werden in mehrere Abschnitte gegliedert. Im ersten Diskussionsteil werden die wesentlichen Unterschiede zwischen den STEMI-Patienten mit und den STEMI-Patienten ohne $\mathrm{CTO}_{\text {NIRA }}$ erörtert. Der zweite Teil widmet sich ausschließlich dem Kollektiv der STEMIPatienten mit zusätzlich chronischem Verschluss und geht detailliert auf den Koronarstatus dieser Patienten und die Auswirkungen auf die Prognose ein. Im abschließenden dritten Diskussionsteil wird dann die mögliche Bedeutung dieser Ergebnisse für die Risikobewertung bei STEMI-Patienten diskutiert. 


\subsection{STEMI-Patienten mit und ohne zusätzlich chronischen Verschluss}

\subsubsection{Gefäßerkrankungstyp und prognostische Bedeutung}

In der vorliegenden Untersuchung wurden unselektioniert 1.247 Patienten mit der Erstdiagnose STEMI eingeschlossen. Der Großteil dieser Patienten konnte der Gruppe MVD -CTO ${ }_{\text {NIRA }}(55 \%)$, gefolgt von der Gruppe SVD (27\%) und der Gruppe MVD $+\mathrm{CTO}_{\text {NIRA }}(10 \%)$ zugeordnet werden. Bei den restlichen STEMI-Patienten wurde entweder keine relevante KHK diagnostiziert oder es konnte keine Koronarangiographie durchgeführt werden. Fast zwei Drittel aller STEMI-Patienten präsentierten sich demnach mit einer Mehrgefäßerkrankung, jeder zehnte STEMI-Patient wies zusätzlich zum akuten Verschluss einen chronischen Verschluss in einem Nicht-Infarkt-Gefäß auf. Von den STEMI-Patienten in der Hochrisikosituation eines kardiogenen Schocks war sogar jeder fünfte Patient von einem $\mathrm{CTO}_{\text {NIRA }}$ betroffen. Ein $\mathrm{CTO}_{\text {NIRA }}$ ist damit eine häufige Erscheinung bei STEMI-Patienten im klinischen Alltag und bedarf dementsprechend einer besonderen Beachtung.

Der Anteil der Patienten mit einem $\mathrm{CTO}_{\mathrm{NIRA}}$ ist in der vorliegenden Untersuchung konkordant mit den Ergebnissen aus anderen Studien, bei denen der CTO $_{\text {NIRA }}$-Anteil zwischen 8,0 - 13,3 \% lag (Moreno et al. 2006; Lexis et al. 2011; Bataille et al. 2012b; Claessen et al. 2012; Tajstra et al. 2012; Hoebers et al. 2013). Es ist allerdings zu beachten, dass die genannten Studien ausschließlich STEMI-Patienten mit CTO $_{\text {NIRA }}$ und erfolgter PCI berücksichtigten, während in der vorliegenden Untersuchung alle Patienten mit STEMI erfasst wurden. Hier wurden somit auch solche Patienten eingeschlossen, bei denen trotz STEMI-Diagnose keine PCI erfolgte.

Auffällig ist im Vergleich zu einigen Studien, dass der Anteil an Patienten mit MVD in der vorliegenden Untersuchung an der Göttinger Klinik deutlich höher lag. Dies ist vor allem damit zu begründen, dass einige Vergleichsstudien eine relevante Stenosierung im Sinne einer Koronargefäßerkrankung erst ab einer Lumeneinengung von $>70 \%$ werteten (Claessen et al. 2009; Bataille et al. 2012b; Tajstra et al. 2012), während sowohl in der vorliegenden als auch in anderen Untersuchungen eine Stenosierung bereits ab einer Lumeneinengung von $50 \%$ als relevant bezeichnet wurde (Van der Schaaf et al. 2006; Claessen et al. 2012). Dies hatte zur Folge, dass in der vorliegenden Untersuchung auch Patienten mit vergleichsweise geringer Stenosierung der Gruppe MVD zugeordnet wurden.

Als wesentliches Ergebnis im Vergleich der Patientengruppen mit und ohne zusätzlich chronischen Verschluss fand sich ein signifikanter Unterschied in der Kurzzeit- 
sterblichkeit. Es konnte gezeigt werden, dass STEMI-Patienten mit einem CTO $_{\text {NIRA }}$ im Vergleich zu den STEMI-Patienten ohne $\mathrm{CTO}_{\mathrm{NIRA}}$ ein mehr als dreifach erhöhtes Risiko aufwiesen, im Krankenhaus zu versterben. Die Krankenhaussterblichkeit lag in der vorliegenden Untersuchung bei STEMI-Patienten mit SVD bei 4,7\%, in der Gruppe MVD -CTO NIRA $_{\text {v }}$ verstarben 9,8 \% der Patienten, während in der Gruppe MVD +CTO NIRA 26,8 \% der Patienten im Krankenhaus verstarben. Die Unterschiede in der Sterblichkeit zwischen den einzelnen Patientengruppen sind dabei tendenziell vergleichbar mit Ergebnissen aus anderen Veröffentlichungen (Claessen et al. 2012; Tajstra et al. 2012; Hoebers et al. 2013). Zusammengefasst liegt die Kurzzeitsterblichkeit (Krankenhaus bis 30Tage) in diesen drei Studien bei STEMI-Patienten mit $\mathrm{CTO}_{\mathrm{NIRA}}$ bei 17,2 \% und bei STEMI-Patienten ohne CTO $_{\text {NIRA }}$ bei 4,7 \% (O'Connor et al. 2015).

Auffällig ist, dass die Krankenhaussterblichkeit in der vorliegenden Untersuchung sowohl bei STEMI-Patienten mit CTO $_{\text {NIRA }}$ als auch bei STEMI-Patienten ohne $\mathrm{CTO}_{\text {NIRA }}$ insgesamt vergleichsweise hoch erscheint. Ein Erklärungsansatz für die höhere Sterblichkeit in der Universitätsmedizin Göttingen liegt dabei vor allem in der Risikokonstellation der Patienten - so werden in der UMG verhältnismäßig viele Hochrisikopatienten behandelt. Der durchschnittliche TIMI Risk Score aller Patienten der UMG lag im Studienzeitraum bei 4,14 Punkten während er z. B. in der FITT-STEMI-Gesamtgruppe ( $\mathrm{n}=23.532$ Patienten, exkl. Patienten der UMG) aktuell bei 3,83 Punkten liegt (bisher unveröffentlichte Daten; Stand: 27.11.2016). Dieses Bild präsentiert sich auch mit Blick auf spezielle Risikogruppen wie z. B. beim Anteil an STEMI-Patienten mit kardiogenem Schock oder prähospitaler Reanimation. Während der Anteil an Schockpatienten in der UMG bei 16 \% und der Anteil prähospital reanimierter Patienten bei $11 \%$ aller STEMI-Patienten lag, beträgt aktuell in der FITT-STEMI-Gesamtgruppe (exkl. Patienten der UMG) der Anteil an Schockpatienten $11 \%$ und der Anteil an prähospital reanimierten Patienten $8 \%$ (bisher unveröffentlichte Daten; Stand 27.11.2016). Als eine der wenigen Kliniken der SupraMaximalversorgung in der Region, inklusive eines Zentrums für Thorax-, Herz- und Gefäßchirurgie, ist die UMG auch überregional eine Anlaufstelle für Hochrisikopatienten mit komplexen kardiologischen Krankheitsbildern. Dies spiegelt sich auch im großen Einzugsgebiet der Patienten und dem hohen Anteil an Patienten mit Sekundärtransport (34 \% aller STEMI-Patienten), ein Großteil davon per Rettungshubschrauber, wider (siehe Kapitel 4.1.2).

\subsubsection{Einfluss von Komorbiditäten und Alter}

Der zugrundeliegende Mechanismus, der zu der schlechten Prognose bei STEMI-Patienten mit $\mathrm{CTO}_{\text {NIRA }}$ führt, ist in weiten Teilen unbekannt. In der Literatur wird von einem 
multifaktoriellen Geschehen ausgegangen, dabei werden mehrere potentielle Erklärungen für das deutlich erhöhte Sterblichkeitsrisiko bei STEMI-Patienten mit CTO $_{\text {NIRA }}$ aufgeführt (Lexis et al. 2011; Claessen et al. 2012; O’Connor et al. 2015). Eine Ursache für das schlechte Outcome dieser Patienten wird beim Betrachten der hier erhobenen Basischarakteristika im Vergleich der verschiedenen Patientengruppen sichtbar. STEMIPatienten mit $\mathrm{CTO}_{\mathrm{NIRA}}$ wiesen insgesamt ein erhöhtes Risiko und darüber hinaus vermehrt Komorbiditäten auf, beides einhergehend mit einer schlechteren Prognose (siehe u. a. auch (Lexis et al. 2011; Claessen et al. 2012; Tajstra et al. 2012)). So war in der vorliegenden Untersuchung der durchschnittliche TIMI Risk Score im Vergleich der einzelnen Patientengruppen in der Gruppe MVD $+\mathrm{CTO}_{\text {NIRA }}$ am höchsten. Mehr als jeder zweite STEMI-Patient mit MVD $+\mathrm{CTO}_{\mathrm{NIRA}}$ hatte einen TIMI Risk Score von $\geq 5(54 \%)$, in der Gruppe der Patienten mit MVD -CTO ${ }_{\text {NIRA }}$ waren es lediglich $42 \%$. Die Unterschiede zwischen diesen beiden Gruppen waren dabei statistisch signifikant.

Des Weiteren waren Patienten mit MVD $+\mathrm{CTO}_{\text {NIRA }}$ älter, hatten signifikant häufiger eine 3-Gefäß-KHK und häufiger einen Myokardinfarkt sowie eine PCI in der Vorgeschichte. Zudem hatten Patienten mit MVD $+\mathrm{CTO}_{\text {NIRA }}$ häufiger eine Niereninsuffizienz oder einen Zustand nach Apoplex.

\subsubsection{Kardiovaskuläre Risikofaktoren}

Mit Blick auf die einzelnen kardiovaskulären Risikofaktoren hat sich vor allem beim Anteil der Patienten mit Diabetes mellitus ein deutlicher, signifikanter Unterschied zwischen den Gruppen MVD + und -CTO ${ }_{\text {NIRA }}$ gezeigt. Während in der Gruppe MVD -CTO ${ }_{\text {NIRA }}$ lediglich jeder fünfte Patient einen diagnostizierten DM aufwies, war in der Gruppe der Patienten mit $\mathrm{CTO}_{\text {NIRA }}$ jeder dritte Patient ein Diabetiker. Insgesamt wiesen $17 \%$ der STEMIPatienten mit Diabetes mellitus einen $\mathrm{CTO}_{\text {NIRA }}$ auf. Einhergehend mit diesen Ergebnissen zeigen Studien, dass Patienten mit DM ein sehr hohes Risiko für besonders schwere Gefäßerkrankungen aufweisen (Mak et al. 1997; Claessen et al. 2010).

Im Gegensatz dazu war bei den Risikofaktoren Hyperlipidämie, arterielle Hypertension und positive Familienanamnese die Prävalenz in der Patientengruppe MVD - $\mathrm{CTO}_{\text {NIRA }}$ höher. Auffällig ist zudem, dass unter den Patienten der Gruppe MVD $+\mathrm{CTO}_{\text {NIRA }}$ deutlich weniger Raucher zu finden waren. Der Anteil an Patienten mit Raucheranamnese war, wie bereits von Claessen et al. (2012) beschrieben, bei den Patienten mit SVD am höchsten und nahm mit zunehmender Schwere der Gefäßerkrankung sukzessive ab. Bezüglich des Risikofaktors Adipositas konnte kein relevanter Unterschied zwischen den einzelnen Patientengruppen festgestellt werden, im Vergleich der Gruppen zeigte der durchschnittliche BMI nur geringfügige Unterschiede. Des Weiteren lag, entgegen den 
Ergebnissen von Bataille et al. (2013), nach denen die CTO-Prävalenz in beiden Geschlechtern nahezu identisch war, in der vorliegenden Untersuchung die Prävalenz eines $\mathrm{CTO}_{\text {NIRA }}$ bei den Männern mit STEMI mit $11 \%$ deutlich höher als bei den Frauen mit $6 \%$. Der Anteil männlicher Patienten war in der Gruppe MVD $+\mathrm{CTO}_{\text {NIRA }}$ signifikant höher als in der Gruppe ohne $\mathrm{CTO}_{\mathrm{NIRA}}(80 \%$ vs. $71 \%$, p = 0,036). Vergleichbar mit Claessen et al. (2012) war in der vorliegenden Untersuchung mit steigendem Ausmaß der Gefäßerkrankung ein signifikanter Anstieg des Anteils männlicher Patienten an der jeweiligen Gruppe zu verzeichnen.

\subsubsection{Reduzierte LV-Funktion}

Eine weitere mögliche Erklärung für die hohe Krankenhaussterblichkeit bei STEMIPatienten mit MVD $+\mathrm{CTO}_{\mathrm{NIRA}}$ findet sich bei der Analyse der im Krankenhaus erfassten linksventrikulären Ejektionsfraktion. Während es zwischen den Patientengruppen SVD und MVD - $\mathrm{CTO}_{\text {NIRA }}$ keinen Unterschied im Anteil der STEMI-Patienten mit einer LVEF von $\leq 30 \%$ gab (SVD: $5 \%$; MVD -CTO ${ }_{\text {NIRA }}$ : $\%$ ), fand sich in der Gruppe der Patienten mit MVD $+\mathrm{CTO}_{\text {NIRA }}$ bei $14 \%$ aller Patienten ein LVEF $\leq 30 \%$. Der Unterschied zwischen den Gruppen MVD -CTO ${ }_{\text {NIRA }}$ und MVD + CTO $_{\text {NIRA }}$ war dabei hoch signifikant, womit das Vorliegen eines $\mathrm{CTO}_{\mathrm{NIRA}}$ offenbar in einem ganz besonderen Maße einen negativen Einfluss auf die LV-Pumpfunktion hat. Leider war es nicht möglich, im Rahmen dieser Arbeit die LVEF aller STEMI-Patienten vollständig zu ermitteln, sodass ein großer Teil $(27 \%$ aller STEMI-Patienten) dieser Daten fehlt. Etwas stärker fällt dies in der Patientengruppe mit MVD $+\mathrm{CTO}_{\text {NIRA }}$ ins Gewicht. Hier fehlt, vor allem bedingt durch die hohe Krankenhaussterblichkeit in dieser Patientengruppe (teilweise verstarben Patienten periprozedural), bei $29 \%$ der Patienten die LVEF. Diese Daten können somit lediglich einen Trend aufzeigen, werden aber durch ebenfalls signifikante Ergebnisse von Claessen et al. (2009) gestützt. Obwohl hier bei einem noch größeren Teil der Patienten (47\%) die LVEF fehlte, zeigte sich an einer repräsentativen Kohorte, dass zum einen die Anwesenheit eines $\mathrm{CTO}_{\mathrm{NIRA}}$ mit einer LVEF $\leq 40 \%$ assoziiert ist und zum anderen sich diese LVEF im Verlauf eines Jahres nach STEMI überaus stark im Sinne eines negativen Remodellings verschlechtert.

Eine linksventrikuläre Dysfunktion wird als eine der Hauptursachen für die hohe Sterblichkeit nach einem Myokardinfarkt angesehen (Norris et al. 1984; Halkin et al. 2005a). In diesem Zusammenhang weisen viele Patienten mit einem CTO bereits im Vorfeld eines akuten STEMI eine LV-Dysfunktion, entweder im Rahmen der bestehenden Infarktanamnese oder aber aufgrund der chronischen Ischämie im CTO-Areal (Sachdeva et al. 2014), auf (Christofferson et al. 2005; Carlino et al. 2015). Christofferson et al. (2005) 
wiesen dabei bei CTO-Patienten eine signifikant verringerte Ejektionsfraktion im Vergleich zu KHK-Patienten ohne CTO nach. Im Rahmen eines akuten Infarktes haben diese CTOPatienten mit bereits vorbestehender LV-Dysfunktion dann weniger Möglichkeiten, die akute weitere Abnahme der LVEF ausreichend zu kompensieren (Tajstra et al. 2012). Dies führt zu der beschriebenen starken Reduktion der LVEF bei STEMI-Patienten mit MVD $+\mathrm{CTO}_{\text {NIRA }}$ und damit einhergehend zu einem erhöhten Sterblichkeitsrisiko.

\subsubsection{Schock und präklinische Reanimation}

All diese Komponenten und Mechanismen (Risikofaktoren, Komorbiditäten und die eingeschränkte LVEF) zusammen führen im Rahmen eines STEMI bei CTO-Patienten unter anderem deutlich häufiger zu einem kardiogenen Schock und/oder cardiac arrest mit Reanimationspflichtigkeit (O'Connor et al. 2015). In der vorliegenden Untersuchung war der Anteil der Hochrisikopatienten mit Schock sowie mit präklinischer Reanimation in der Gruppe MVD $+\mathrm{CTO}_{\mathrm{NIRA}}$ signifikant höher als in der Vergleichsgruppe MVD -CTO ${ }_{\text {NIRA }}$. Jeder dritte STEMI-Patient mit $\mathrm{CTO}_{\text {NIRA }}$ befand sich beim Eintreffen im Herzkatheterlabor im kardiogenen Schock, zudem wurden $17 \%$ aller STEMI-Patienten mit $\mathrm{CTO}_{\text {NIRA }}$ präklinisch reanimiert. Im Einklang mit Van der Schaaf et al. (2010) und Bataille et al. (2012a) konnte darüber hinaus gezeigt werden, dass die $\mathrm{CTO}_{\mathrm{NIRA}}$-Patienten im kardiogenen Schock eine signifikant höhere Sterblichkeit aufwiesen als Schockpatienten mit MVD -CTO ${ }_{\text {NIRA }}$ respektive mit SVD (61\% vs. $41 \%$ vs. $21 \%$ ). Die Hochrisikosituation des kardiogenen Schocks wird somit mutmaßlich durch das Vorhandensein eines CTO $_{\text {NIRA }}$ zusätzlich extrem negativ beeinflusst.

\subsubsection{Erfolgsrate der Akutintervention}

Als weitere interessante Beobachtung kommt hinzu, dass, wie bereits von Tajstra et al. (2012) berichtet, die primäre PCI bei STEMI-Patienten mit MVD $+\mathrm{CTO}_{\text {NIRA }}$ signifikant seltener erfolgreich ist. Ein TIMI-III-Fluss im akuten Infarktgefäß konnte in der vorliegenden Untersuchung lediglich bei $88 \%$ der STEMI-Patienten mit MVD +CTO wiederhergestellt werden, während in den anderen Patientengruppen dieser Wert bei $91 \%$ (Patienten mit SVD) bzw. $94 \%$ (Patienten mit MVD -CTO ${ }_{\text {NIRA }}$ ) lag. Auch dieses Ergebnis trägt möglicherweise zu der schlechteren Prognose bei STEMI-Patienten mit CTO $_{\text {NIRA }}$ bei, da ein TIMI-Fluss < III bei STEMI nach PCI mit einer erhöhten Sterblichkeitsrate einhergeht (Ross et al. 1993). 


\subsection{STEMI-Patienten mit zusätzlich chronischem Verschluss: Bedeutung von Koronarstatus, Kollateralversorgung und früherem Infarkt}

Im folgenden Teil der Diskussion liegt der Fokus ausschließlich auf STEMI-Patienten mit zusätzlich chronisch verschlossenem Nicht-Infarkt-Gefäß. Von den 123 STEMI-Patienten mit $\mathrm{CTO}_{\text {NIRA }}$ fand sich bei 15 Patienten mehr als ein von einem CTO $_{\text {NIRA }}$ betroffenes Gefäß. Vergleichbar mit Bataille et al. (2012b) wies diese kleine Gruppe von STEMIPatienten mit MVD+>1CTO ${ }_{\text {NIRA }}$ mit 33,3\% das allerhöchste Krankenhaussterblichkeitsrisiko auf. Aufgrund der geringen Fallzahl wird diese Gruppe im Folgenden jedoch nicht berücksichtigt, es werden ausschließlich STEMI-Patienten analysiert, bei denen ein zusätzlich chronischer Verschluss in lediglich einem Nicht-Infarkt-Gefäß vorlag.

\subsubsection{Vorkommen eines $\mathrm{CTO}_{\mathrm{NIRA}}$ in den unterschiedlichen Koronargefäßen}

In der vorliegenden Untersuchung konnte bei insgesamt 108 STEMI-Patienten ein zusätzlich chronischer Verschluss in einem Nicht-Infarkt-Gefäß identifiziert werden. Bei knapp der Hälfte dieser Patienten war der $\mathrm{CTO}_{\text {NIRA }}$ in der RCA lokalisiert (48\%), deutlich seltener befand sich der $\mathrm{CTO}_{\mathrm{NIRA}} \operatorname{im} \operatorname{RCX}(31 \%)$ sowie im RIVA (21\%). Diese Ergebnisse bestätigen die Resultate aus vorhergehenden Untersuchungen, in denen ebenfalls die RCA deutlich häufiger von einem $\mathrm{CTO}_{\mathrm{NIRA}}$ betroffen war als der RCX bzw. der RIVA (Lexis et al. 2011; Bataille et al. 2012b; Claessen et al. 2012).

Da vermutet wird, dass die meisten chronischen Verschlüsse aus akuten Gefäßverschlüssen auf Basis des Pathomechanismus eines Myokardinfarktes hervorgehen (Guo et al. 2013; Irving 2014), wäre anzunehmen, dass die Prävalenz von chronischen und akuten Verschlüssen in den einzelnen Gefäßen vergleichbar ist. Dies ist mit Blick auf das akute Infarktgefäß im Rahmen einer primären PCI allerdings nicht der Fall: Bei $41 \%$ aller Patienten mit PCI wurde in der vorliegenden Untersuchung die RCA akut rekanalisiert, während der RIVA bei $44 \%$ aller STEMI-Patienten das Zielgefäß war.

Für diese Diskrepanz in der Prävalenz zwischen STEMI- und CTO-Gefäß gibt es eine mögliche, auf der Kollateralversorgung basierende Erklärung. So konnte in verschiedenen Studien gezeigt werden, dass eine schnelle Rekrutierung von Kollateralen zumeist bei akuten Infarkten der RCA stattfindet, wobei der RIVA in diesem Fall am häufigsten die Donor-Arterie ist (Antoniucci et al. 2002; Elsman et al. 2004). Dies hätte zur Folge, dass sich bei Patienten mit akutem RCA-Infarkt aufgrund der schnellen Kollateralversorgung des betroffenen Gebietes häufiger keine relevante Infarzierung bemerkbar macht und sich der Verschluss so chronisch manifestieren kann. Patienten mit einem akuten RIVA-Infarkt hingegen rekrutieren deutlich seltener frühe Kollateralen und haben zudem insgesamt eine 
schlechtere Prognose (Stone et al. 1988; Nienhuis et al. 2009), womit diese Patienten vermutlich häufiger keinen chronischen Verschluss ausbilden und darüber hinaus häufiger bereits in der Frühphase versterben. Im Rahmen dieser Arbeit fanden sich dabei bei Patienten mit $\mathrm{CTO}_{\text {NIRA }}$ im Stromgebiet des RIVA häufiger pathologische Q-Zacken (QZacken bei $52 \%$ aller STEMI-Patienten mit CTO $_{\text {NIRA }}$ im RIVA), die auf einen abgelaufenen alten Infarkt hinweisen, als bei Patienten mit $\mathrm{CTO}_{\mathrm{NIRA}}$ in der RCA (Q-Zacken bei $38 \%$ aller STEMI-Patienten mit CTO $_{\text {NIRA }}$ in der RCA), was die hier aufgestellte Hypothese stützen würde. Mit Blick auf den Anteil an Patienten mit allgemein Hinweisen auf einen abgelaufenen alten Infarkt (Q-Zacken und/oder Infarktanamnese) gleichen sich allerdings die Anteile in beiden Gruppen fast an, wobei aber auch hier Patienten mit $\mathrm{CTO}_{\text {NIRA }}$ im RIVA etwas häufiger Hinweise auf einen alten Infarkt erkennen lassen (52\% bei $\mathrm{CTO}_{\text {NIRA }}$ im RIVA vs. $48 \%$ bei $\mathrm{CTO}_{\text {NIRA }}$ in der RCA).

\subsubsection{Prognostische Bedeutung der Lokalisation des CTO $_{\text {NIRA }}$ in den unterschiedlichen Koronargefäßen}

Als ein wesentliches Ergebnis konnte in der vorliegenden Untersuchung gezeigt werden, dass die Kurzzeitsterblichkeit bei STEMI-Patienten stark vom betroffenen $\mathrm{CTO}_{\mathrm{NIRA}^{-}}-\mathrm{Gefäß}$ abhängt. Die höchste Krankenhaussterblichkeit fand sich mit 30,8 \% bei Patienten mit $\mathrm{CTO}_{\text {NIRA }}$ in der RCA, während STEMI-Patienten mit CTO $_{\text {NIRA }}$ im RCX (21,2\%) und im RIVA (21,7\%) vergleichsweise seltener im Krankenhaus verstarben. Innerhalb von 30 Tagen verstarb sogar bereits jeder dritte STEMI-Patient mit einem CTO $_{\text {NIRA }}$ in der RCA $(32,7 \%)$, dagegen blieb in den Gruppen mit $\mathrm{CTO}_{\mathrm{NIRA}}$ im RIVA und im RCX die Sterblichkeit innerhalb dieses Zeitraums unverändert.

Die hohe Sterblichkeit von STEMI-Patienten mit $\mathrm{CTO}_{\text {NIRA }}$ in der RCA lässt sich möglicherweise mit dem akuten Infarkt im Stromgebiet der linken Koronararterie und dessen Einfluss auf die LV-Funktion erklären. Definitionsgemäß muss bei einem CTO $_{\text {NIRA }}$ der RCA der STEMI im Verlauf der linken Koronararterie liegen. Ein STEMI der Vorderwand, verursacht durch einen akuten Verschluss des RIVA, hat hierbei bekannterweise eine besonders schlechte Prognose (Stone et al. 1988; Nienhuis et al. 2009). Dies konnte auch in der hier untersuchten Gesamtgruppe bestätigt werden, die Krankenhaussterblichkeit aller Göttinger STEMI-Patienten mit PCI ( $\mathrm{n}=990$ Patienten) lag dabei abhängig vom Zielgefäß bei 10,3 \% bei Akutverschluss im RIVA, bei 8,1 \% im RCX und bei 7,7\% in der RCA. Zudem wurde im Rahmen dieser Arbeit ergänzend auch der mögliche Einfluss des akuten STEMI-Gefäßes auf die Prognose bei ausschließlich $\mathrm{CTO}_{\mathrm{NIRA}}$-Patienten analysiert. Um das STEMI-Gefäß exakt zuordnen zu können, wurde diese Analyse jedoch nur bei STEMI-Patienten durchgeführt, bei denen auch tatsächlich 
eine primäre PCI des Akutverschlusses vorgenommen worden war. Dabei konnte gezeigt werden, dass auch in dieser Kohorte akute Verschlüsse des RIVA, aber hier auch des RCX, bei Vorhandensein eines $\mathrm{CTO}_{\mathrm{NIRA}}$ mit einer deutlich erhöhten Krankenhaussterblichkeit (28,6 \% bzw. 26,3 \%) einhergehen, während Patienten mit akuten Verschlüsse der RCA ein vergleichsweise geringes Risiko (16,1\%) aufwiesen. Bei der Interpretation dieser Ergebnisse muss allerdings beachtet werden, dass durch die Auswahl der Patienten mit durchgeführter primärer PCI eine große Zahl an Patienten mit außerordentlich schwerer Gefäßerkrankung (und z. B. Behandlung mit Akut-Bypass-OP) nicht berücksichtigt wurde.

Die insgesamt schlechte Prognose bei einem akuten RIVA-Verschluss lässt sich u. a. mit dem Anteil des über das jeweilige Koronargefäß versorgten Myokards erklären. Laut Leaman et al. (1981) erfolgen bei einem Rechtsversorgungstyp $16 \%$ der Myokardversorgung des linken Ventrikels über die RCA und $84 \%$ über die LCA, 2/3 davon übernimmt der RIVA. Dabei wird deutlich, dass prognostische Aussagen hinsichtlich einzelner Verschlusslokalisationen nur unter Berücksichtigung des zugrundeliegenden Versorgungstyps wirklich relevant sind. Diesbezüglich konnte in der vorliegenden Untersuchung gezeigt werden, dass STEMI-Patienten mit einem $\mathrm{CTO}_{\mathrm{NIRA}}$ in der RCA im Falle eines Rechtsversorgungstyps $(n=42)$ mit 33,3\% eine enorm hohe Krankenhaussterblichkeit aufwiesen, während von den Patienten mit $\mathrm{CTO}_{\text {NIRA }}$ in der RCA mit bestehendem Linksversorgungstyp $(\mathrm{n}=10)$ lediglich 20,0 \% im Krankenhaus verstarben. Unter zusätzlicher Berücksichtigung des akuten STEMI-Gefäßes lag im Einklang mit dem Anteil an der Myokardversorgung in der vorliegenden Arbeit die Kurzzeitsterblichkeit innerhalb von 30 Tagen bei STEMI-Patienten mit CTO $_{\text {NIRA }}$ in der RCA bei Rechtsversorgungstyp und erfolgter PCI bei akutem Verschluss des RIVA mit $33 \%$ deutlich höher als bei einem akuten Verschluss des RCX mit lediglich $22 \%$.

Gerade akute Gefäßverschlüsse der LCA bei zusätzlich chronischem Verschluss der rechten Koronararterie scheinen bei Patienten mit Rechtsversorgungstyp, mutmaßlich durch einen globalen Einfluss auf Vorder- und Hinterwand des linken sowie auch auf den rechten Ventrikel einen besonders negativen Einfluss auf die Pumpleistung zu haben und das Herz im besonderen Maße zu schwächen. Hier ist vor allem die Kombination aus Akutverschluss des RIVA und zusätzlich chronischem Verschluss der RCA bei Rechtsversorgungstyp mit einem besonders hohen Sterblichkeitsrisiko assoziiert.

Bei einem Linksversorgungstyp hingegen wird der komplette linke Ventrikel über die LCA versorgt, dabei werden ca. 42 \% der Blutversorgung über den RCX gewährleistet (Sianos et al. 2005). Im Rahmen der vorliegenden Arbeit wiesen insgesamt lediglich elf von 108 STEMI-Patienten mit CTO $_{\text {NIRA }}(10 \%)$ einen Linksversorgungstyp auf, die restlichen 97 Patienten wurden der Gruppe Rechtsversorgungstyp (hier Rechts- und kodominanter 
Versorgungstyp zusammengefasst) zugeordnet. Dieses Verhältnis zwischen Links- und Rechtsversorgungstyp ist dabei vergleichbar zu früheren Angaben in der Literatur (Veltman et al. 2015). Aufgrund dieser geringen Anzahl an Patienten mit Linksversorgungstyp können zu dieser Kohorte in der vorliegenden Untersuchung keine verlässlichen Aussagen getroffen werden.

In einer erst kürzlich veröffentlichten Studie aus den Niederlanden wurde erstmals in der Literatur der Einfluss der Lokalisation des chronischen Verschlusses auf die Prognose von STEMI-Patienten untersucht (Hoebers et al. 2016). Nach multivariater Analyse war in dieser Arbeit bei 480 STEMI-Patienten mit CTO $_{\text {NIRA }}$ und erfolgter primärer PCI ein chronischer Verschluss des proximalen RIVA (von den Autoren wurde dabei Segment 6 und 7 als proximal gewertet) sowie ein akuter Verschluss des RIVA und des proximalen RCX (bei Vorhandensein eines CTO $_{\text {NIRA }}$ ) mit einem höheren Sterblichkeitsrisiko assoziiert. Aufgrund der möglichen Kombinationen zwischen CTO- und STEMI-Gefäß kamen die Autoren zu der Bewertung, dass fast alle STEMI-Patienten mit CTO $_{\text {NIRA }}$ ein erhöhtes Sterblichkeitsrisiko aufweisen.

In dieser Untersuchung aus den Niederlanden wurde allerdings keine spezielle SubGruppenanalyse bezogen auf den Koronarversorgungstyp und das exakte CTO $_{\text {NIRA }^{-}}$ Gefäßsegment durchgeführt - es fand lediglich eine Unterteilung der Verschlusslokalisation in proximalen und distalen RIVA bzw. RCX, nicht aber der RCA, statt. Zudem fand hierbei der zugrundliegende Versorgungstyp keine Berücksichtigung. Pauschale Aussagen zum Outcome eines bestimmten vom CTO $_{\text {NIRA }}$ betroffenen Koronargefäßes bei STEMI sind ohne Berücksichtigung des kompletten individuellen Koronarstatus nach den Ergebnissen der vorliegenden Untersuchung jedoch nur bedingt verwertbar.

Dabei ergeben sich, wie die im Rahmen dieser Promotionsarbeit erstmals präsentierten und im folgenden diskutierten Ergebnisse zeigen, aus bestimmten Kombinationen des vom $\mathrm{CTO}_{\text {NIRA }}$ betroffenen Segments und des zugrundeliegenden Koronarversorgungstyps spezielle Untergruppen mit einer zum einen extrem schlechten, zum anderen aber auch relativ guten Prognose. Die detaillierte Analyse der $\mathrm{CTO}_{\text {NIRA-Lokalisation mit Blick auf das }}$ Koronarsegment und den Versorgungstyp wird daher in den folgenden Kapiteln weiter fokussiert diskutiert.

\subsubsection{Prognostische Bedeutung der Lokalisation des CTO $_{\mathrm{NIRA}}$ in unterschiedlichen Koronargefäßsegmenten}

Detaillierte Untersuchungen im Rahmen dieser Arbeit zeigten, dass nicht nur das vom $\mathrm{CTO}_{\text {NIRA }}$ betroffene Gefäß an sich, sondern vor allem auch die CTO-Lokalisation im 
jeweiligen Gefäßabschnitt des Koronargefäßes einen besonderen Einfluss auf die Prognose von STEMI-Patienten hat.

In der Literatur finden sich bezüglich der exakten CTO-Lokalisation bei STEMI-Patienten nur sehr limitierte Informationen. Lediglich in einer Arbeit wurde bisher die Prävalenz eines chronischen Verschlusses bei STEMI-Patienten in den einzelnen Koronarsegmenten, allerdings ohne Ergebnisse zur prognostischen Bedeutung, bestimmt. Dabei befand sich am häufigsten mit einem Anteil von $24 \%$ ein chronischer Verschluss in der proximalen RCA (Lexis et al. 2011). Im Rahmen der hier vorliegenden Untersuchung war ein $\mathrm{CTO}_{\text {NIRA }}$ ebenfalls am häufigsten in der proximalen RCA (30\% aller CTOs) gefolgt von der medialen RCA (11\%), dem proximalen RCX (10\%) und dem proximalen RIVA (9\%) lokalisiert - zusammengefasst befand sich hier knapp die Hälfte aller CTO $_{\text {NIRA }}$ in proximalen Gefäßabschnitten. Die Prävalenz nahm dabei mit zunehmender Entfernung vom jeweiligen Koronarostium immer weiter ab, sodass sich in den peripheren Gefäßsegmenten lediglich $15 \%$ aller CTO $_{\text {NIRA }}$ bei STEMI-Patienten befanden.

Im Rahmen der vorliegenden Untersuchung konnte erstmals gezeigt werden, dass die Entfernung des chronischen Verschlusses zum Koronarostium eine besondere prognostische Bedeutung bei STEMI-Patienten besitzt. Mit 32,1 \% präsentierte sich dabei die höchste Krankenhaussterblichkeit bei STEMI-Patienten mit einem CTO $_{\text {NIRA }}$ in proximalen Gefäßabschnitten. Waren hingegen die drei anderen, weiter distal gelegenen, Abschnitte von einem chronischen Verschluss betroffen, so lag die Krankenhaussterblichkeit nur noch bei jeweils ca. $20 \%$.

Eine mutmaßliche Erklärung für diese hohe Krankenhaussterblichkeit bei STEMIPatienten mit einem $\mathrm{CTO}_{\mathrm{NIRA}}$ in einem proximalen Gefäßsegment liegt in einem insgesamt vergrößerten Ischämie- oder, je nach Ausmaß des ggf. früheren Infarktes, Narbenareal. Wie bereits erwähnt, entspricht das distal eines CTO gelegene Myokardareal einer chronischen Ischämiezone (Sachdeva et al. 2014). Zudem muss davon ausgegangen werden, dass bei Patienten mit CTO und Hinweisen auf einen früheren Infarkt ein gewisser Anteil des CTO-Areals bereits vernarbt und nicht mehr lebensfähig ist (Carlino et al. 2015). Je weiter proximal nun das Koronargefäß chronisch verschlossen ist, umso größer ist mutmaßlich das distal dieses Verschlusses gelegene potentiell ischämische bzw. vernarbte Myokardareal. Im Falle eines STEMI existiert damit neben dem akut betroffenen ischämischen Myokard ein weiteres großes Ischämie- oder Narbenareal mit besonders negativem Einfluss auf die Ventrikelfunktion. Lexis et al. (2011) fanden dahingehend bei STEMI-Patienten mit $\mathrm{CTO}_{\mathrm{NIRA}}$ ein größeres akut infarziertes Areal im Vergleich zu STEMI-Patienten ohne $\mathrm{CTO}_{\text {NIRA }}$. Die Autoren haben diesen Unterschied in der Infarktgröße allerdings nicht hinsichtlich der $\mathrm{CTO}_{\mathrm{NIRA}}$-Lokalisation im Gefäß untersucht. 
Auch der besondere Einfluss eines chronischen Verschlusses in einem proximalen Gefäßabschnitt auf eine potentielle Kollateralversorgung hat vermutlich eine große Bedeutung für die schlechte Prognose bei diesen STEMI-Patienten.

Im Rahmen eines akuten Verschlusses hat eine frühzeitige Kollateralisierung des Infarktgebietes einen positiven Effekt auf das Outcome der Patienten (Ishihara et al. 2005). Bei einem schon im Vorfeld bestehenden CTO bieten sich im Falle eines akuten Verschlusses in einem anderen Koronargefäß allerdings weniger Möglichkeiten einer raschen Kollateralisierung des akut ischämischen Gebietes. Aufgrund des chronischen Verschlusses steht eines von zwei möglichen Kollateral-Donor-Gefäßen nur eingeschränkt zur Verfügung (Lexis et al. 2011; O’Connor et al. 2015). Je weiter proximal sich der $\mathrm{CTO}_{\text {NIRA }}$ befindet, umso kleiner ist die potentielle Donor-Gefäßstrecke für das Areal des akuten Verschlusses, und umso geringer ist die Wahrscheinlichkeit einer schnellen Kollateralisierung von diesem Gefäß aus. Dem akuten Infarktareal stehen in diesem Fall weniger Kollateralverbindungen für einen ausreichenden Blutfluss zur Verfügung, was einen zusätzlich negativen Einfluss auf das Ausmaß des akuten Ischämiegebietes und die Infarktgröße hätte.

Darüber hinaus konnte mit Blick auf eine schon bestehende Kollateralisierung des chronischen Verschlusses, allerdings bei Patienten ohne akuten STEMI, elegant gezeigt werden, dass unter Stressbedingungen durch eine Kollateralversorgung besonders großer Myokardareale der Blutfluss auch direkt in der Kollateral-Donor-Arterie negativ beeinflusst wird. Hierbei kam es infolge einer verminderten fraktionellen Flussreserve in der DonorArterie zu einer ausgeprägten Ischämie in diesem Areal. Eine erfolgreiche Rekanalisation des kollateralisierten CTO-Gefäßes führte, bei in diesen Fällen vorliegender mittelgradiger Stenosierung der Donor-Arterie, häufig zu einer Reduzierung des Ausmaßes der Ischämie im Donor-Gefäß-Areal (Sachdeva und Uretsky 2011; Sachdeva et al. 2013). Im Falle eines akuten STEMI der Donor-Arterie könnte dies mutmaßlich bedeuten, dass das Ausmaß der Ischämie bzw. Infarzierung im akut betroffenen Areal vor allem bei bestehender Kollateralisierung großer Myokardabschnitte, wie es vermutlich bei proximalen CTOs der Fall ist, erheblich negativ beeinflusst wird.

Die aus diesen möglichen Pathomechanismen resultierende großflächige Myokardunterversorgung bei Patienten mit chronischem Verschluss in einem proximalen Gefäßabschnitt führt mutmaßlich verstärkt zu einer globalen LV-Dysfunktion mit negativem Einfluss auf die Hämodynamik. Als Folge können hierbei zusätzlich Schock und Arrhythmien auftreten und dadurch ein erhöhtes periinfarzielles Sterblichkeitsrisiko mit sich bringen. So präsentierten sich in der vorliegenden Untersuchung die Patienten mit 
proximal gelegenem $\mathrm{CTO}_{\mathrm{NIRA}}$ auch deutlich häufiger im kardiogenen Schock als Patienten mit einem $\mathrm{CTO}_{\text {NIRA }}$ in den übrigen Gefäßabschnitten zusammengefasst (40 \% vs. $24 \%$ ).

\subsubsection{Besondere prognostische Bedeutung eines CTO $_{\text {NIRA }}$ der proximalen RCA bei Rechtsversorgungstyp}

Bei der Analyse der einzelnen Gefäßsegmente unter Berücksichtigung des zugrundeliegenden Versorgungstyps präsentierte sich mit Blick auf die Prognose eine besondere Hochrisikogruppe in der Kohorte der STEMI-Patienten mit chronischem Verschluss. So konnte die allerhöchste Kurzzeitsterblichkeit in der Gruppe der STEMI-Patienten mit einem $\mathrm{CTO}_{\mathrm{NIRA}}$ im proximalen Segment der RCA (= Segment 1) bei vorliegendem Rechtsversorgungstyp identifiziert werden - mit insgesamt 26 Patienten betraf dies ca.

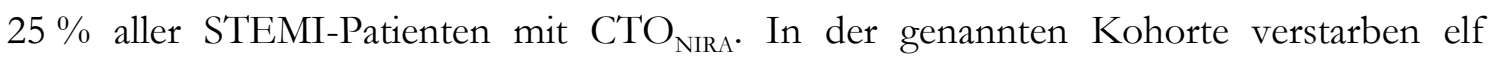
Patienten (42,3\%) im Krankenhaus, die Sterblichkeit innerhalb von 30 Tagen lag sogar bei 46,2 \% ( $n=12$ Patienten). Im Vergleich mit allen anderen möglichen $\mathrm{CTO}_{\text {NIRA }^{-}}$ Lokalisationen zusammengefasst $(n=82)$ zeigte sich hinsichtlich der Kurzzeitsterblichkeit ein statistisch signifikanter Unterschied (Tod im Krankenhaus: 42,3\% vs. 20,7 \%; p = 0,029). Dabei konnten bis auf das Alter (STEMI-Patienten mit CTO $_{\text {NIRA }}$ in RCA-Segment 1 mit Rechtsversorgung waren interessanterweise deutlich jünger als die Patienten der Vergleichsgruppe) und das vom akuten Infarkt betroffene Gefäß (Grund für den deutlichen Unterschied zwischen diesen beiden Gruppen ist vermutlich das Fehlen der RCA als Infarktgefäß bei den Patienten mit CTO $_{\text {NIRA }}$ in Segment 1 der RCA) keine weiteren statistisch signifikanten Unterschiede zwischen diesen Gruppen ermittelt werden.

Um den Einfluss dieser speziellen $\mathrm{CTO}_{\mathrm{NIRA}^{-}}$Lokalisation auf die Krankenhaussterblichkeit weiter zu eruieren, wurden ergänzend zwei unterschiedliche Regressionsmodelle zur multivariaten Analyse der Sterblichkeitsprädiktoren in der Gruppe der STEMI-Patienten mit $\mathrm{CTO}_{\mathrm{NIRA}}$ erstellt. In diesen Modellen präsentierte sich ein $\mathrm{CTO}_{\mathrm{NIRA}}$ in Segment 1 der RCA bei Rechtsversorgungstyp als signifikant unabhängiger Prädiktor der Krankenhaussterblichkeit (Modell nach Vortest: OR 6,01; 95 \% CI: 1,08 - 33,53; $\mathrm{p}=$ 0,041; backward stepwise: OR 5,08; 95 \% CI: 1,15 - 22,54; $\mathrm{p}=$ 0,032). Die STEMI-Lokalisation im EKG war dagegen auf Grundlage dieses Modells in der vorliegenden Kohorte kein unabhängiger Prädiktor der Krankenhaussterblichkeit, womit zumindest im Rahmen dieser Untersuchung der mutmaßliche negative Einfluss eines Vorderwandinfarktes auf die Prognose der STEMI-Patienten mit $\mathrm{CTO}_{\text {NIRA }}$ keine führende Rolle spielte.

Bei STEMI-Patienten mit $\mathrm{CTO}_{\mathrm{NIRA}}$ in Segment 1 der RCA scheinen sich die bereits genannten negativen Einflüsse eines proximalen CTO und die negativen Einflüsse der RCA als CTO-Gefäß unter den besonderen Bedingungen des Rechtsversorgungstyps zu 
potenzieren. Aus dieser Kombination resultiert die extrem schlechte Prognose in dieser Patientengruppe.

Dieses besonders eindrucksvolle Ergebnis der Patientengruppe mit zusätzlich chronischem Verschluss in Segment 1 der RCA bei Rechtsversorgungstyp hätte bei Bestätigung in größeren Kohorten eine enorme Relevanz für die Risikobewertung von STEMI-Patienten - dies bedeutet andererseits aber auch, dass nicht per se, wie in anderen Untersuchungen dargelegt, alle STEMI-Patienten mit einem zusätzlich chronischen Verschluss eine besonders schlechte Prognose haben. Das Resultat dieser vorliegenden Untersuchung unterstreicht dabei ganz wesentlich die Bedeutung der Erfassung des kompletten Koronarstatus inklusive Versorgungstyp und exakter Verschlusslokalisation bei Patienten mit chronischen Koronargefäßverschlüssen. Im Rahmen der Literaturrecherche fand sich dabei bis dato keine Untersuchung die den Koronarstatus in der Gruppe der STEMI-Patienten mit $\mathrm{CTO}_{\text {NIRA }}$ so eingehend analysiert hat.

In den weiteren Untergruppen der einzelnen Gefäßsegmente befanden sich, mit Ausnahme des genannten proximalen RCA-Segments, zu wenige Patienten, um verlässliche Aussagen treffen zu können. Hier lässt sich aus der vorliegenden Untersuchung lediglich ein Trend ablesen, der erst in Untersuchungen an wesentlich größeren Patientenkollektiven bestätigt werden kann.

\subsubsection{Bedeutung der Kollateralversorgung bei CTO NIRA $_{\text {I }}$}

Insgesamt gesehen ist das Kollateralnetzwerk bei CTO-Patienten von großer Bedeutung, da das distal eines chronischen Verschlusses gelegene Myokardareal wahrscheinlich nur über dieses Kollateralnetz metabolisch versorgt und so vor einem Zelluntergang bewahrt wird. In der vorliegenden Untersuchung konnte, wie bereits in der Literatur bei CTOPatienten (ohne ACS) beschrieben (Werner 2014), bei einem Großteil der STEMIPatienten mit $\mathrm{CTO}_{\text {NIRA }}(85 \%)$ eine angiographisch sichtbare Kollateralisierung (Rentrop Score 2 - 3) nachgewiesen werden. Dabei konnte gezeigt werden, dass die kleine Gruppe der Patienten ohne sichtbare Kollateralisierung des chronisch verschlossenen Gefäßes (Rentrop Score 0 - 1) mit einer Krankenhaussterblichkeit von $44 \%$ offenbar ein deutlich höheres Risiko aufwies als die Patientengruppe mit einer angiographisch sichtbaren Kollateralversorgung des chronischen Verschlusses (Krankenhaussterblichkeit $23 \%$ ). Trotz der in dieser Gruppe nur sehr kleinen Patientenzahl fand sich dabei statistisch ein deutlicher Trend $(p=0,078)$. Eine fehlende angiographisch sichtbare Kollateralisierung des Areals distal eines CTO könnte als Hinweis darauf gewertet werden, dass in dem abhängigen Myokardareal bereits eine relevante Myokardnarbe vorliegt, was im Falle eines akuten 
STEMI in einem anderen Koronargefäß dann durch die zusätzliche Ischämie- bzw. Nekrosezone zu einem wesentlich größeren Funktionsausfall des Myokards führt.

Hinsichtlich der bestehenden Kollateralisierung ist es allerdings aufgrund interindividueller Unterschiede bei zum Teil mehreren Kollateralpfaden schwierig, allgemeingültige Aussagen zu treffen. Zudem muss davon ausgegangen werden, dass viele Kollateralverbindungen im Rahmen einer Angiographie aufgrund des begrenzten Auflösungsvermögens gar nicht erst detektiert wurden.

\subsubsection{Besondere Bedeutung einer Kollateralisierung über das Infarktgefäß als Donor-Arterie}

Die prognostische Bedeutung einer möglichen Interaktion zwischen CTO- und AkutInfarkt-Areal über potentielle Kollateralen ist derzeit unklar.

In der vorliegenden Untersuchung entsprang bei der Hälfte der STEMI-Patienten mit $\mathrm{CTO}_{\text {NIRA }}$ und angiographisch sichtbarer interkoronarer Kollateralisierung eine Kollaterale direkt aus dem vom akuten Infarkt betroffenen Gefäß als Donor-Gefäß. In diesem Setting war die Krankenhaussterblichkeit bei Patienten mit Kollateralversorgung des CTO ausgehend vom Infarktgefäß mit 21,2 \% vergleichbar hoch wie bei Patienten mit Kollateralversorgung ausgehend von einem Nicht-Infarkt-Gefäß mit 21,9\%. Auch nach Bataille et al. (2013) gab es hinsichtlich des Kollateralursprungs keine Unterschiede in der Prognose zwischen STEMI-Patienten mit oder ohne Kollateralisierung des CTO $_{\text {NIRA }}$ über das akute Infarktgefäß. Laut Werner (2014) sind bei über $90 \%$ der CTO-Patienten mit guter Kollateralversorgung die Kollateralen in Stress- und Belastungssituationen unter anderem aufgrund eines coronary steals (Perfusionsabnahme im kollateralisierten Myokard bei Stress (Gould 1989)), bedingt durch eine Stenose der Donor-Arterie oder durch eine beeinträchtigte vasodilatatorische Reserve der Mikrozirkulation, nicht in der Lage, einen adäquaten Blutfluss aufrechtzuerhalten. Zu der speziellen Situation eines STEMI mit $\mathrm{CTO}_{\text {NIRA }}$ existieren diesbezüglich jedoch keine Daten. Es ist aber durchaus vorstellbar, dass in der besonderen (systemischen) Stresssituation eines akuten Myokardinfarkts auch das kollateralisierte Myokard, unabhängig davon, ob die Donor-Arterie direkt vom STEMI betroffen ist, in einem großen Umfang von einer Ischämie im Sinne eines coronary steals bedroht sein könnte. Der Impact des akuten Infarktes wäre damit um einiges größer, dies könnte die hohe Sterblichkeit auch bei $\mathrm{CTO}_{\mathrm{NIRA}^{-}}$-Patienten mit Kollateralversorgung über das Nicht-Infarktgefäß erklären.

Bezüglich der Kollateralversorgung des CTO über das vom akuten Infarkt betroffene Gefäß als Donor-Arterie wird in der Literatur der sogenannte double jeopardy effect, bei dem der Ursprung der Kollaterale distal eines akuten Verschlusses liegt und somit erst nach 
erfolgreicher PCI sichtbar wird, als eine weitere mögliche Ursache für die allgemein erhöhte Sterblichkeit bei STEMI-Patienten mit CTO $_{\text {NIRA }}$ diskutiert (Lexis et al. 2011; Claessen et al. 2012; O’Connor et al. 2015). Dabei lautet die Hypothese, dass in diesem Fall neben der IRA auch zusätzlich das über dieses Infarktgefäß kollateralversorgte CTO-Areal direkt vom akuten Infarkt betroffen ist und sich damit das akute Ischämieareal über die IRA hinaus wesentlich vergrößert. In einer erst kürzlich veröffentlichten Studie konnte diesbezüglich gezeigt werden, dass STEMI-Patienten, deren $\mathrm{CTO}_{\mathrm{NIRA}}-\mathrm{Gefäß}$ über eine Kollaterale mit Ursprung aus dem vom Infarkt betroffenen Gefäß distal des akuten Verschlusses versorgt wurde $(\mathrm{n}=56)$, mit 46,5\% eine deutlich höhere 30-Tage-Sterblichkeit aufwiesen als alle anderen STEMI-Patienten mit CTO $_{\text {NIRA }}(\mathrm{n}=104)$ mit 4,8 \% (Şen et al. 2016). Diese Ergebnisse würden die Hypothese des double jeopardy effects stützen, konnten allerdings in der vorliegenden Untersuchung in keiner Weise bestätigt werden. Hier verstarben in der Gruppe der STEMI-Patienten mit CTO $_{\text {NIRA }}$ und Kollateralabgang aus dem vom Infarkt betroffenen Gefäß interessanterweise deutlich mehr Patienten bei einem Kollateralabgang proximal des akuten Verschlusses (Krankenhaussterblichkeit 33,3 $\%)$ als bei einem Kollateralabgang distal des akuten Verschlusses (Krankenhaussterblichkeit $6,7 \% ; \mathrm{p}=0,062)$. Zu berücksichtigen ist allerdings, dass von den insgesamt 87 STEMI-Patienten mit $\mathrm{CTO}_{\text {NIRA }}$ und durchgeführter primärer PCI bei nur $87 \%$ ein TIMI-III-Fluss nach PCI im Zielgefäß hergestellt werden konnte. Bei einem kleinen Teil dieser STEMI-Patienten mit $\mathrm{CTO}_{\text {NIRA }}$ und primärer PCI konnte eine potentielle Kollateralisierung distal des akuten Verschlusses somit nur eingeschränkt erfasst werden, da das Infarktgefäß nicht adäquat wiedereröffnet werden konnte. Die Diskrepanz in den Ergebnissen könnte zudem durch die geringe Patientenzahl in beiden Untersuchungen und zusätzlich auch durch Unterschiede in den Behandlungs- bzw. Rekanalisierungszeiten der betroffenen STEMI-Arterie bedingt sein (während die contact-to-ballon-Zeit in der vorliegenden Studie bei STEMI-Patienten mit CTO $_{\text {NIRA }}$ im Median bei 114 min lag, wurden die Behandlungszeiten in der Studie von Sen et al. nicht erfasst).

Es ist aber durchaus vorstellbar, dass eine Kollateralverbindung zwischen dem Gefäßabschnitt distal des akuten Verschlusses und distal des chronischen Verschlusses einen gewissen protektiven Effekt für das akut ischämische Infarktgebiet bietet. So wird bei einem Großteil der CTO-Patienten der chronische Verschluss netzwerkartig über multiple Kollateralen versorgt, bei $86 \%$ aller Patienten mit einem chronischen Verschluss fanden Werner et al. (2003) mehr als nur einen Kollateralpfad zum CTO-Gebiet. Es ist daher denkbar, dass das CTO-Gebiet beispielsweise zusätzlich über Brückenkollateralen versorgt wird und durch eine Flussumkehr, ausgelöst durch eine Änderung der Druckverhältnisse im Koronarsystem bei STEMI, in der bereits angelegten Kollateralverbindung zwischen 
IRA und CTO-Gefäß ein retrograder Blutfluss mit dann protektivem Effekt zum akuten Infarktgebiet ermöglicht wird. Auf diesem Wege könnte die Versorgung des Infarktgebietes möglicherweise, wenn auch deutlich vermindert, aufrechterhalten werden - dies könnte besonders bei einer schnellen Wiedereröffnung des Infarktgefäßes, wie hier bei einer C2BZeit von 114 min der Fall, sehr myokard-protektiv wirken.

Zusätzlich ist es möglich, dass ein Kollateralabgang proximal des akuten Verschlusses den Blutfluss im Donor-Gefäß negativ beeinflusst und dem akuten Infarktgebiet einen Teil der eigentlichen Blutversorgung und damit Perfusionsdruck entzieht. So wurde bereits in einem nicht akuten Rahmen eine verminderte fraktionelle Flussreserve in der KollateralDonor-Arterie bei bestehender Kollateralversorgung von großen chronisch verschlossenen Arealen beobachtet (Sachdeva und Uretsky 2011; Sachdeva et al. 2013). Bei einem Kollateralabgang distal des akuten Verschlusses würde dieser Effekt nicht auftreten und somit mutmaßlich eine bessere Prognose ermöglichen.

Diese hier aufgestellten Hypothesen müssten an einem deutlich größeren STEMI-Kollektiv mit ebenso einheitlicher und exakter Analyse der Kollateralversorgung geprüft werden. Die hier präsentierten Ergebnisse zeigen, dass es auch unter Berücksichtigung der Kollateralversorgung zum einen Risikogruppen mit einer besonders hohen Kurzzeitsterblichkeit gibt, und zum anderen in der Kollateralversorgung möglicherweise auch ein gewisser prognostischer Vorteil liegt. Daraus folgt, dass auch mit Blick auf die Kollateralversorgung nicht jeder STEMI-Patient mit CTO $_{\text {NIRA }}$ per se eine schlechte Prognose aufweist.

\subsubsection{Dokumentierter früherer Infarkt}

Nach Werner et al. (2003) wiesen CTO-Patienten ohne einen dokumentierten früheren Infarkt ein besonders stark ausgeprägtes Kollateralnetz auf. Diese Kollateralen waren offenbar schon im Vorfeld angelegt, konnten sich direkt nach dem akuten Gefäßverschluss entfalten und so in der akuten Verschlusssituation eine relevante Infarzierung verhindern und die LV-Funktion aufrechterhalten. Bei Patienten mit einem dokumentierten Infarkt hingegen konnte sich das Kollateralnetz mutmaßlich nicht schnell genug entwickeln, um einen Infarkt effektiv zu verhindern. Hier entstand ein adäquates Kollateralnetz wahrscheinlich erst im weiteren Verlauf nach dem Infarktereignis.

Im Rahmen der vorliegenden Untersuchung stellte sich die Frage, ob CTO-Patienten ohne dokumentierten früheren Infarkt im Rahmen eines akuten STEMI u. a. aufgrund einer ggf. besseren Kollateralrekrutierung eine günstigere Prognose aufwiesen als CTO-Patienten mit einem dokumentierten früheren Infarkt. Insgesamt konnte bei $41 \%$ der STEMI-Patienten mit $\mathrm{CTO}_{\text {NIRA }}(\mathrm{n}=44)$ elektrokardiographisch und/oder anamnestisch ein Hinweis auf einen abgelaufenen alten Infarkt ermittelt werden (Q-Zacken über dem CTO-Areal 
und/oder anamnestisch Zustand nach Myokardinfarkt). Dabei war der Anteil an Patienten mit angiographisch sichtbaren Kollateralen in den Gruppen mit und ohne Hinweis auf einen früheren Infarkt vergleichbar. Laut Werner et al. (2003) entwickelt sich ein ausgeprägtes Kollateralnetz vor allem im Verlauf von 2 - 12 Wochen, womit sich möglicherweise im Laufe der Zeit die Kollateralentwicklung bei CTO-Patienten mit und ohne dokumentiertem Infarkt angleichen konnte.

Von den STEMI-Patienten mit CTO $_{\text {NIRA }}$ wies in der vorliegenden Untersuchung insgesamt jeder dritte Patient pathologische Q-Zacken in den auf den CTO zu beziehenden EKGAbleitungen auf, dabei wurden am häufigsten Q-Zacken bei chronischen Verschlüssen im RIVA (52\%) respektive der RCA (38\%) identifiziert. Fast drei Viertel aller Q-Zacken fanden sich in den proximalen und medialen Segmenten des RIVA und der RCA offenbar gehen vor allem chronische Verschlüsse in Segmenten, die eine große Myokardmasse versorgen, häufiger mit Q-Zacken einher. Diese Ergebnisse sind vergleichbar mit anderen Untersuchungen zu STEMI-Patienten mit $\mathrm{CTO}_{\mathrm{NIRA}}$ (Lexis et al. 2011; Bataille et al. 2012b).

Die Ergebnisse der vorliegenden Arbeit zeigen, dass STEMI-Patienten mit CTO $_{\text {NIRA }}$ und dokumentiertem früheren Infarkt eine höhere Krankenhaussterblichkeit (31,8 \%) aufwiesen als Patienten ohne Hinweis auf einen alten Infarkt (21,9 \%). Die mögliche rasche Kollateralrekrutierung konnte zwar mutmaßlich in diesen Fällen mit bestehendem CTO und akutem STEMI, möglicherweise auch bedingt durch den CTO, eine Infarzierung nicht verhindern, wirkte aber offenbar dennoch in gewissen Maßen protektiv. Hierbei muss berücksichtigt werden, dass Patienten mit dokumentiertem Infarkt aufgrund des bereits abgelaufenen alten Infarktes vermutlich schon im Vorfeld des STEMI eine stärker eingeschränkte LV-Pumpfunktion und damit schlechtere Prognose aufwiesen als CTOPatienten ohne dokumentierten früheren Infarkt.

Es bleibt festzuhalten, dass STEMI-Patienten mit $\mathrm{CTO}_{\mathrm{NIRA}}$ und einem dokumentierten alten Infarkt eine besondere Risikogruppe in der Infarktversorgung darstellen. STEMIPatienten mit einem Hinweis auf einen alten Myokardinfarkt bei CTO $_{\text {NIRA }}$ im RIVA verstarben dabei mit 41,7 \% signifikant häufiger im Krankenhaus als STEMI-Patienten mit einem chronischen Verschluss im RIVA ohne Hinweis auf einen abgelaufenen alten Infarkt (Krankenhaussterblichkeit 0 \%). Im Falle eines STEMI könnte somit eine Q-Zacke über der Vorderwand ein Hinweis auf eine besonders schlechte Prognose sein. 


\subsection{Risikobewertung bei STEMI-Patienten}

Die Behandlung von STEMI-Patienten im Rahmen einer Koronarintervention bietet neben dem eigentlichen therapeutischen Zweck auch Möglichkeiten der individuellen Risikostratifikation basierend auf dem Koronarstatus. Risikoscores ermöglichen es, auf Basis verschiedener Variablen komplizierte medizinische Gegebenheiten objektivierbar in Form einer dem Schweregrad der vorliegenden Erkrankung entsprechenden Punktzahl darzustellen, um somit das individuelle Patientenrisiko besser bewerten zu können. Abgesehen von der Bedeutung im klinischen Alltag für die individuelle Therapieentscheidung finden Scoringsysteme u. a. auch als Werkzeug in klinischen Studien und in der Qualitätssicherung Anwendung (Ohmann und Gross-Weege 1992; Ohmann und Gross-Weege 1993; Neugebauer und Bouillon 1994; Ohmann und Lefering 2002).

\subsubsection{Etablierte Risikoscores bei STEMI-Patienten}

Die aktuellen Leitlinien zum Management des STEMI erwähnen zur Risikostratifikation bisher lediglich Risikoscores, die bereits initial vor der primären PCI erhoben werden können und somit den Koronarstatus nicht berücksichtigen (Steg et al. 2012). Im klinischen Alltag spielen hierbei vor allem der TIMI Risk Score (TRS), ein Score basierend auf acht klinischen und elektrokardiographischen Parametern unter anderem zur Abschätzung der 30-Tage-Sterblichkeit (Morrow et al. 2000) sowie der GRACE Score (Fox et al. 2006), ebenfalls basierend auf klinischen und elektrokardiographischen Variablen, eine Rolle. Diese Scores erlauben es, Patienten direkt bei der Ankunft im Krankenhaus in bestimmte Risikogruppen einzuteilen und die folgende Therapie an das Risikoprofil anzupassen. Der GRACE Score kommt vor allem im Rahmen eines NSTEMI zum Einsatz und besitzt hier eine direkte therapeutische Konsequenz (Roffi et al. 2016), dagegen wird beim STEMI und den hier klar definierten Behandlungsabläufen dem TRS eine größere Bedeutung zugeschrieben (Lev et al. 2008). Allerdings wurde der TRS bereits in der präPCI Ära im Rahmen von Lysestudien entwickelt. Patienten mit hohem Risiko die als ungeeignet für eine Lysetherapie gewertet wurden, wurden somit in diesen Studien nicht berücksichtigt (Morrow et al. 2000). Solche Hochrisikopatienten sind daher auch bei der Evaluierung des TRS nicht mit in die Analysen einbezogen worden. Darüber hinaus war bei STEMI in Vergleichsstudien die PCI der Lysetherapie hinsichtlich Rekanalisationserfolg und Überlebensrate deutlich überlegen, sodass eine Prognosebewertung nach dem TRS für STEMI im Zeitalter der primären PCI problematisch erscheint.

Neben diesen klinisch-elektrokardiographischen Risikoscores ist es nach erfolgter Koronarangiographie möglich, Risikokonstellationen basierend auf dem Koronarstatus nach PCI zu erheben. Verbreitet ist z. B. der Gensini Score, mit dessen Hilfe das Ausmaß der koronaren 
Herzkrankheit anhand der Koronaranatomie, -morphologie und des Stenosegrads ermittelt werden kann (Gensini 1983). Daneben ist der SYNTAX Score ein weiteres nennenswertes Werkzeug zur Beurteilung des Koronarstatus (Sianos et al. 2005). Dieser Score wurde entwickelt, um der Komplexität von Mehrgefäßerkrankungen gerecht zu werden, da die Einteilung in 1-, 2- oder 3-Gefäßerkrankung zu oberflächlich ist und die eigentliche Heterogenität der Gefäßläsionen nur wenig realitätsnah widergespiegelt wird. Ein hoher Score weist auf eine besonders schwere Gefäßerkrankung und damit einhergehend auf eine diffizile Therapie und somit letztlich auch auf eine schlechtere Prognose hin (Windecker et al. 2014). Ein CTO ist im Algorithmus des SYNTAX-Scores der stärkste Risikomultiplikator und daher Ausdruck einer besonders komplexen Gefäßerkrankung. Obwohl der SYNTAX Score ursprünglich nur für Patienten mit 3-Gefäßerkrankung unter dem Aspekt elektiver Interventionen entwickelt wurde, konnten Studien zeigen, dass der Score auch für die Risikoeinschätzung im Rahmen eines STEMI verwendet werden kann und dabei als unabhängiger Prädiktor der Mortalität gilt (Garg et al. 2011).

Um sowohl klinische als auch angiographische Risikomerkmale ausreichend zu berücksichtigen und damit ein möglichst allumfassendes Risikoprofil jedes einzelnen Patienten zu ermitteln, erscheint es sinnvoll, einen kombinierten Risikoscore anzuwenden. Hier ist vor allem der CADILLAC Score $\mathrm{zu}$ nennen, der neben klinischen auch angiographische Parameter wie eine mögliche Mehrgefäßerkrankung, die LV-EF sowie den TIMI Grade Flow Post-PCI erfasst (Halkin et al. 2005b). Im Vergleich zu den rein klinisch-basierten Risikoscores wie TIMI und GRACE zeigt sich der CADILLAC Score prognostisch überlegen (Lev et al. 2008), allerdings wurden Hochrisikopatienten mit Schock und komplexer Mehrgefäßerkrankung von der CADILLAC-Studie ausgeschlossen. Mit dem EuroHeart Score wurde zudem ein neueres Instrument zur Risikoabschätzung von Patienten nach erfolgter PCI entwickelt (De Mulder et al. 2011). Dieser Score verknüpft, ähnlich dem CADILLAC Score, klinische und angiographische Variablen. Mit insgesamt sechs angiographischen Merkmalen (TIMI Grade Flow Vor-PCI, Mehrgefäßerkrankung, Hauptstammstenose, proximale RIVA Stenose, Bifurkationsläsion und Typ-C-Läsion) kommt dem Koronarstatus hierbei eine besonders bedeutende Rolle zu. Der Score kann zudem auch speziell zur Risikostratifikation bei STEMI-Patienten nach erfolgreicher PCI angewendet werden.

Mit Blick auf die vorliegende Arbeit lassen die hier präsentierten Ergebnisse erhebliche Zweifel an der Validität des bisher für STEMI-Patienten allgemein präferierten TIMI Risk Score aufkommen. So demonstrieren die erhobenen Daten hinsichtlich der Risikobewertung eindeutig eine Diskrepanz zwischen dem TIMI Risk Score und der Kurzzeitprognose in einzelnen Untergruppen. Während z. B. bei STEMI-Patienten mit einem 
$\mathrm{CTO}_{\text {NIRA }}$ im medialen Abschnitt die Krankenhaussterblichkeit bei vergleichsweise niedrigen 19,0 \% lag, betrug der durchschnittliche TIMI Risk Score in dieser Gruppe 5,43 Punkte und war damit im Vergleich zu den anderen Gefäßabschnitten der höchste aller Werte. Dagegen wies die Gruppe der STEMI-Patienten mit $\mathrm{CTO}_{\mathrm{NIRA}}$ in einem proximalen Gefäßabschnitt bei einer extrem hohen Krankenhaussterblichkeit von 32,1 \% im Durchschnitt einen vergleichsweise geringen TIMI Risk Score von 4,64 Punkten auf. Auch bei den STEMI-Patienten mit der allerhöchsten Sterblichkeit $\left(\mathrm{CTO}_{\mathrm{NIRA}}\right.$ in proximaler RCA bei Rechtsdominanz) war der TIMI Risk Score vergleichsweise niedrig (4,69 Punkte). Die aktuell in den Leitlinien erwähnten klinischen Risikoscores scheinen somit nicht ausreichend $\mathrm{zu}$ sein um vor allem Hochrisikopatienten mit STEMI adäquat zu identifizieren. Dabei erscheint es von großer Bedeutung, einen robusten Risikoscore für STEMI-Patienten zu entwickeln, der neben den klinischen Risikomerkmalen vor allem auch möglichst allumfassend den Koronarstatus, mit besonderem Blick auf chronische Verschlüsse in Nicht-Infarkt-Gefäßen und den zugrundeliegenden Versorgungstyp, berücksichtigt. Ein idealer aber bisher nicht existenter Risikoscore müsste somit in der Lage sein, aus der Gruppe der STEMI-Patienten mit $\mathrm{CTO}_{\mathrm{NIRA}}$ Patienten zu identifizieren, die ein besonders hohes Sterblichkeitsrisiko aufweisen.

Die Einteilung von Patienten in bestimmte Risikogruppen unter Zuhilfenahme von Risikoscores mit daraus hervorgehender Prognoseeinschätzung ist dabei im Hinblick auf eine optimierte Therapie, wie z. B. eine Rekanalisierung des chronisch verschlossenen Gefäßes im Verlauf, aber auch zum Erkennen der Grenzen einer Behandlung von Hochrisikopatienten von größter Bedeutung. In Patienten- und Angehörigengesprächen könnten verlässliche Scores zudem möglichst objektiv die Situation des Patienten erörtern und ein realistisches Bild der Prognose zeichnen. Dies würde einen wichtigen Beitrag zur gemeinsamen Therapieentscheidung leisten. Allerdings dürfen Scores dabei niemals als alleiniges Entscheidungskriterium aufgefasst werden, sondern sollten stets unter Berücksichtigung der Gesamtsituation des individuellen Patienten zu einem allumfassenden Therapiekonzept beitragen.

\subsubsection{Risikobewertung im Rahmen der Qualitätssicherung}

Bei den in dieser Untersuchung erfassten STEMI-Patienten einer universitätsmedizinischen Klinik der Supramaximalversorgung zeigte sich insgesamt eine vergleichsweise schlechte Prognose. So lag die Krankenhaussterblichkeit aller STEMI-Patienten der UMG mit 9,9 \% deutlich höher als beispielsweise im FITT-STEMI-Gesamtprojekt mit 8,2 \% (1.927 von 23.532 Patienten, exkl. Patienten der UMG; unveröffentlichte Daten aus FITT-STEMI; Stand 27.11.2016). Allerdings fällt auf, dass auch der durchschnittliche TIMI Risk Score in 
der Universitätsmedizin Göttingen mit 4,14 Punkten deutlich höher lag als in der FITTSTEMI-Gesamtgruppe mit 3,83 Punkten. Dieser Sachverhalt hat eine enorme Bedeutung für die grundsätzliche Risikobewertung im Rahmen der Qualitätssicherung: Häufig wird ausschließlich das Patientenoutcome als Maßstab für die Qualität der erbrachten Leistung einer Klinik gewertet. Ohne eine Risikoadjustierung, die insbesondere Hochrisikopatienten exakt bewertet und berücksichtigt, schneiden vor allem Kliniken der Supramaximalversorgung in externen Vergleichen besonders schlecht ab. Nur eine auf einem überprüften und validen Risikoscore basierende Qualitätssicherung kann eine realistische und objektive Bewertung der Ergebnisqualität einzelner Zentren erlauben (Ohmann und Lefering 2002). Der TRS ist hier allerdings im Sinne einer adäquaten Prognoseeinschätzung bei Hochrisikopatienten mit STEMI zu ungenau. Mit Blick auf die erhobenen Ergebnisse in dieser Arbeit muss ein Score in der Lage sein, STEMI-Patienten möglichst exakt einzustufen und damit Hochrisikopatienten zu identifizieren, um aussagekräftige Qualitätsvergleiche zu ermöglichen. Die medizinische Qualitätssicherung erhält dabei insgesamt einen immer größeren Stellenwert in der Patientenversorgung, bisher existieren aber keine adäquaten Scores, die speziell Patienten mit STEMI objektiv einstufen. Die hier vorliegenden Ergebnisse sind daher auch für die gesetzliche Qualitätssicherung von größter Bedeutung, bei der bei STEMI-Patienten bisher weder die Angabe $\mathrm{CTO}_{\mathrm{NIRA}}$ noch dessen Lokalisation oder der Versorgungstyp zur Risikobewertung erfasst wird. 


\subsection{Ausblick}

Die hier präsentierten Ergebnisse zeigen eindrucksvoll die große Relevanz eines zusätzlich chronischen Verschlusses in einem Nicht-Infarkt-Gefäß für die Risikobewertung bei STEMI-Patienten.

Dabei fungierte die Datenerfassung dieser Arbeit als eine Art Pilotprojekt. Im Zuge dieser Untersuchung wurde der FIT'T-STEMI-Dokumentationsbogen (siehe Anhang, Kapitel 7.1, Abbildung 46 und 47) aktualisiert und um die Möglichkeit der prospektiven Erfassung chronischer Verschlüsse inklusive Segmentlokalisation erweitert. Dies ermöglicht in Zukunft die Überprüfung der hier gewonnen Ergebnisse an einem großen Kollektiv prospektiv erfasster STEMI-Patienten an über 50 PCI-Zentren in ganz Deutschland.

Eine robuste Bestätigung dieser Daten hätte dabei auch eine besondere Bedeutung für mögliche neue Therapieansätze. Durch Fortschritte im Bereich der Kathetertechniken sowie in der Entwicklung neuer Technologien ist es in Verbindung mit erfahrenen Untersuchern durchaus möglich, chronische Verschlüsse sicher zu rekanalisieren (Carlino et al. 2015). Dabei ist eine sorgfältige Auswahl der Patienten, die für eine auch weiterhin komplexe CTO-PCI in Frage kommen könnten, von größter Bedeutung. Im Rahmen der vorliegenden Untersuchung konnten so STEMI-Patienten identifiziert werden, die möglicherweise in besonderem Maße von einer PCI des chronischen Verschlusses profitieren würden. Hinsichtlich der Koronarintervention gäbe es hierbei zwei mögliche Ansatzpunkte:

Auf der einen Seite könnte eine CTO-PCI bereits im Vorfeld eines STEMI präventiv wirken. So könnte bei CTO-Patienten mit einer Ischämiesymptomatik und nachgewiesenem vitalen Myokard durchaus eine Indikation zur elektiven Rekanalisation des chronischen Verschlusses gestellt werden (Sianos et al. 2012). Befände sich, wie in dieser Arbeit gezeigt, ein CTO in einem proximalen Gefäßabschnitt bzw. in der RCA bei einem Rechtsversorgungstyp, würde dies im Falle eines akuten STEMI zu einer außerordentlich schlechten Prognose führen. Auch ist davon auszugehen, dass sich unter der großen Zahl an prähospital verstorbenen STEMI-Patienten ein gewisser Anteil an Patienten mit einem solchen Koronarstatus befindet. Bei all diesen Patienten hätte eine elektive CTO-PCI neben einer Verbesserung der Symptomatik und LVEF eventuell einen zusätzlich protektiven Effekt für den Fall eines akuten Infarkts im weiteren Verlauf. Claessen et al. (2013) untersuchten bei KHK-Patienten ohne ACS den Einfluss des Zielgefäßes einer CTO-PCI auf die Langzeitsterblichkeit. Sie kamen dabei zu dem Ergebnis, dass eine erfolgreiche PCI eines CTO im RIVA bzw. im RCX mit einer verringerten Langzeitsterblichkeit einhergeht, eine erfolgreiche PCI eines CTO in der RCA aber keinen Über- 
lebensvorteil mit sich bringt. Demnach würde man eine CTO-Läsion der RCA eher als relativ benigne einstufen und dementsprechend zurückhaltender rekanalisieren. Mit den in der vorliegenden Arbeit präsentierten Ergebnissen konnte aber gezeigt werden, dass ein CTO in der RCA durchaus von großer Bedeutung für die Prognose sein kann. So war im Falle eines STEMI die Krankenhaussterblichkeit bei Patienten mit CTO $_{\text {NIRA }}$ in der RCA besonders hoch. In diesem Setting präsentiert sich ein chronischer Verschluss der RCA als eine extrem maligne Läsion. Eine erfolgreiche CTO-PCI der RCA würde somit zwar laut Claessen et al. keinen direkten Überlebensvorteil bringen, könnte aber im Vorfeld eines potentiellen STEMI bei Risikopatienten präventiv wirken und damit die Prognose dieser Patienten gegebenenfalls erheblich positiv beeinflussen.

Zum anderen stellt sich die Frage nach der optimalen Akut-Behandlung von STEMIPatienten mit $\mathrm{CTO}_{\mathrm{NIRA}}$. Die aktuellen Leitlinien empfehlen bisher bei hämodynamisch stabilen STEMI-Patienten ausschließlich eine primäre PCI des Infarktgefäßes (Steg et al. 2012). Aufgrund der erhöhten Krankenhaus- und 30-Tage-Sterblichkeit könnte aber eine frühzeitige erfolgreiche Therapie des $\mathrm{CTO}_{\text {NIRA }}$ einen Einfluss auf die Prognose dieser STEMI-Patienten haben (O'Connor et al. 2015). Eventuell würde sich hier bei ausgewählten STEMI-Patienten mit besonders hohem Sterblichkeitsrisiko, also vor allem Patienten mit $\mathrm{CTO}_{\mathrm{NIRA}}$ in proximalen Abschnitten bzw. in der RCA, auch eine zusätzliche Rekanalisation des chronischen Verschlusses während der primären PCI anbieten. Allerdings ist gerade im Rahmen eines akuten STEMI davon auszugehen, dass bei einer potentiellen primären Mehrgefäß-PCI die Erfolgsrate einer CTO-Revaskularisierung insgesamt schlechter ausfallen würde und die Kombination aus speziellem CTO-Draht und voll antikoaguliertem Patienten ein weitaus größeres Interventions- und KontrastmittelNephropathierisiko mit sich bringt (Mukherjee und Roffi 2012). Bisher existieren zu dieser speziellen Situation bei STEMI-Patienten mit $\mathrm{CTO}_{\mathrm{NIRA}}$ kaum robuste Daten. Während Cavender et al. keinen Überlebensvorteil bei Patienten fanden, die im Rahmen der primären PCI bei STEMI mit MVD, aber ohne CTO eine komplette Behandlung aller betroffene Gefäße erfuhren (Cavender et al. 2009), konnten Wald et al. zeigen, dass eine zusätzliche präventive PCI aller Gefäße während der primären PCI bei STEMI mit MVD das Risiko des Auftretens weiterer kardialer Ereignisse signifikant reduziert (Wald et al. 2013). Bezugnehmend auf STEMI-Patienten mit CTO $_{\text {NIRA }}$ kamen Gruppen aus Italien (Valenti et al. 2014) sowie aus China (Yang et al. 2013) im Rahmen kleinerer Studien mit nur sehr geringen Patientenzahlen zu dem Ergebnis, dass eine erfolgreiche CTO-PCI innerhalb von 30 Tagen im Anschluss an die primäre STEMI-PCI (sog. staged PCI) mit einem verbesserten Langzeitüberleben assoziiert sei. Die erst kürzlich veröffentlichte EXPLORE-Studie konnte diese Ergebnisse, erstmals an einem randomisierten Patienten- 
kollektiv (STEMI-Patienten mit $\mathrm{CTO}_{\text {NIRA }}$ und staged CTO-PCI innerhalb von sieben Tagen vs. STEMI-Patienten mit CTO $_{\text {NIRA }}$ aber ohne CTO-PCI) untersucht, nicht bestätigen (Henriques et al. 2016). Es konnte kein Vorteil mit Blick auf die Endpunkte (LVEF und major adverse coronary events nach 4 Monaten) bei den Patienten der CTO-PCI Gruppe ermittelt werden. Lediglich in einer Untergruppe (Patienten mit $\mathrm{CTO}_{\mathrm{NIRA}}$-Lokalisation im RIVA) konnte eine signifikant erhöhte LVEF nach CTO-PCI im Vergleich zu der Gruppe ohne CTO-PCI eruiert werden. Insgesamt weist diese Studie aber einige Limitationen auf (geringe Patientenzahl ( $\mathrm{n}=304$ ), geringe CTO-PCI Erfolgsrate von $73 \%$, teilweise Ausschluss von Hochrisikopatienten mit z. B. Schock oder prähospitaler Reanimation). Hier müssen dringend weitere Untersuchungen erfolgen, die vor allem auch Hochrisikopatienten einschließen, um ein klares Therapiekonzept bei den STEMIPatienten mit $\mathrm{CTO}_{\text {NIRA }}$ zu gewährleisten.

Mit Blick auf die präsentierten Ergebnisse sollte jedoch, unabhängig von der Frage des Zeitpunkts der CTO-Intervention, die Indikation zur Therapie des CTO $_{\text {NIRA }}$ wie bereits erwähnt maßgeblich von einer individuellen Risikobewertung mit Berücksichtigung des detaillierten Koronarstatus aber auch der allgemeinen Patientensituation abhängen.

\subsection{Limitationen}

Die Patienten für die vorliegende Single-Center-Untersuchung wurden im Rahmen des FITT-STEMI-Projektes rekrutiert. Die Basisdaten der STEMI-Patienten wurden dabei prospektiv auf der Grundlage eines Dokumentationsbogens systematisch erfasst. Es muss beachtet werden, dass für das Projekt ausschließlich STEMI-Patienten registriert wurden, die lebend die Universitätsmedizin Göttingen erreicht haben. Zudem wurden für die vorliegende Auswertung STEMI-Patienten mit Zustand nach Koronarbypass-Operation von der Analyse ausgeschlossen. Es kann somit nicht ausgeschlossen werden, dass sich aufgrund des mutmaßlich höheren Risikoprofils gerade unter den prähospital verstorbenen STEMI-Patienten, wie auch unter den STEMI-Patienten mit CABG, eine relevante Anzahl an Patienten mit $\mathrm{CTO}_{\text {NIRA }}$ befand. Durch diese Selektion konnten somit in der Untersuchung sehr wahrscheinlich nicht alle STEMI-Patienten mit CTO $_{\text {NIRA }}$ in die Analyse einbezogen werden.

Die koronarangiographischen Daten der vorliegenden Untersuchung basieren auf Befunden, die im Rahmen der akuten STEMI-Behandlung erhoben wurden. Dabei ist es möglich, dass in diesem Fall der Stenosegrad einzelner Läsionen überschätzt wurde (Hanratty et al. 2002). Dies könnte gegebenenfalls zu der vergleichsweise günstigen 
Prognose der STEMI-Patienten mit MVD ohne $\mathrm{CTO}_{\mathrm{NIRA}}$ beigetragen haben (Claessen et al. 2009).

Für die vorliegende Analyse wichtige koronarangiographische und elektrokardiographische Informationen, die nicht mit Hilfe des Original-Dokumentationsbogens registriert werden konnten, mussten retrospektiv auf Basis der Katheterbefundberichte und der EKGBefunde erfasst werden. Hierbei wurden diese retrospektiv gewonnenen Daten der STEMI-Patienten mit $\mathrm{CTO}_{\text {NIRA }}$ bei jedem einzelnen Patienten systematisch anhand der Originalbefunde überprüft und weitere Details, wie z. B. die Segmentlokalisation des $\mathrm{CTO}_{\text {NIRA }}$ und die Kollateralisierungspfade, anhand der Katheterfilme erfasst und bewertet. Bei einigen Patienten mit $\mathrm{CTO}_{\mathrm{NIRA}}$ konnte das Verschlussalter allerdings nicht sicher bestimmt werden. Nach sorgfältiger Begutachtung der Verschlussmorphologie und der anamnestischen Angaben wurden dabei alle als true-CTO mit einer angenommenen Verschlussdauer $\geq 3$ Monate charakterisierten Läsionen als CTO $_{\text {NIRA }}$ gewertet.

Des Weiteren ist anzumerken, dass lediglich $81 \%$ aller STEMI-Patienten mit CTO $_{\text {NIRA }}$ tatsächlich mit einer primären PCI behandelt wurden. Die Art der jeweiligen Therapie hatte dabei auch einen Einfluss auf die Prognose. So lag die Krankenhaussterblichkeit der STEMI-Patienten mit CTO $_{\text {NIRA }}$ bei einer Akutbehandlung mit PCI bei 25,3\% und bei den STEMI-Patienten mit $\mathrm{CTO}_{\mathrm{NIRA}}$ ohne durchgeführte primäre PCI bei 28,6 \%.

Der Anteil der erfassten Patienten mit CTO an der Gesamtzahl der STEMI lag ungefähr vergleichbar hoch wie in anderen Studien. Allerdings waren einige Untergruppen der Patienten mit $\mathrm{CTO}_{\text {NIRA }}$ zu klein, um statistisch aussagekräftige Ergebnisse zu erzielen.

Zudem konnten nicht alle Patientendaten vollständig erfasst werden. Es finden sich einige Datenlücken im Bereich des 30-Tage-Follow-ups und der LV-Ejektionsfraktion. Darüberhinaus wurde der Einfluss eines CTO $_{\text {NIRA }}$ bei STEMI auf die Langzeitmortalität im Sinne eines 1-Jahres-Follow-ups in der vorliegenden Arbeit nicht untersucht.

Die aufgezeigten Limitationen machen deutlich, dass die hier präsentierten Ergebnisse künftig multizentrisch und prospektiv an einem größeren STEMI-Kollektiv überprüft und validiert werden müssen. 


\section{Zusammenfassung}

In der vorliegenden Untersuchung wurde bei 1.247 konsekutiven Patienten mit akutem STStrecken-Hebungsinfarkt (STEMI) die Bedeutung eines zusätzlich chronischen Verschlusses in einem Nicht-Infarkt-Gefäß $\left(\mathrm{CTO}_{\text {NIRA }}\right)$ systematisch geprüft.

Übereinstimmend mit früheren Untersuchungen konnte gezeigt werden, dass ein CTO bei STEMI-Patienten nicht selten ist: Jeder zehnte in die Universitätsmedizin Göttingen mit STEMI eingelieferte Patient wies zusätzlich zum Akut-Verschluss einen chronischen Verschluss in einem Nicht-Infarkt-Gefäß auf. STEMI-Patienten mit CTO $_{\text {NIRA }}$ hatten dabei eine deutlich höhere Krankenhaussterblichkeit als STEMI-Patienten mit Mehrgefäßerkrankung ohne $\mathrm{CTO}_{\text {NIRA }}(26,8 \%$ vs. 9,8\%).

Es hat sich gezeigt, dass die Lokalisation des $\mathrm{CTO}_{\text {NIRA }}$ von größter Bedeutung für die Prognose ist. So fand sich bei STEMI-Patienten mit $\mathrm{CTO}_{\mathrm{NIRA}}$ in der RCA sowie bei STEMI-Patienten mit einem CTO $_{\text {NIRA }}$ in einem proximalen Gefäßabschnitt jeweils eine besonders hohe Krankenhaussterblichkeit. Ein chronischer Verschluss in der proximalen RCA bei Rechtsversorgungstyp ging in der vorliegenden Untersuchung mit der weitaus höchsten Sterblichkeit (42,3 \%) einher und konnte als unabhängiger Prädiktor der Krankenhaussterblichkeit bei STEMI-Patienten mit $\mathrm{CTO}_{\text {NIRA }}$ identifiziert werden.

Darüber hinaus spielt auch die Kollateralversorgung bei STEMI-Patienten mit CTO $_{\text {NIRA }}$ eine prognostisch relevante Rolle. Besonderes Interesse galt dabei STEMI-Patienten mit einer Kollateralversorgung des CTO-Gebietes über das vom akuten Infarkt betroffene Koronargefäß als Donor-Arterie. Bemerkenswerterweise wiesen hierbei Patienten mit einem Kollateralabgang im Infarktgefäß proximal des akuten Verschlusses eine deutlich höhere Sterblichkeit auf als Patienten mit Kollateralabgang distal des akuten Verschlusses.

Bei STEMI-Patienten stellt somit ein $\mathrm{CTO}_{\text {NIRA }}$ ein erhebliches zusätzliches Sterblichkeitsrisiko dar. Nach den Ergebnissen dieser Arbeit wird dies jedoch nicht durch etablierte Risikoscores differenziert widergespiegelt. Trotz höchster Krankenhaussterblichkeit hatten Patienten mit $\mathrm{CTO}_{\text {NIRA }}$ in der proximalen RCA bei Rechtsversorgungstyp einen vergleichsweise niedrigen TIMI Risk Score. Künftig sollte bei STEMI-Patienten das Vorhandensein eines zusätzlich chronischen Verschlusses in einem Nicht-Infarkt-Gefäß bei der Risikobewertung berücksichtigt werden. Dabei ist die Erhebung des kompletten Koronarstatus inklusive detaillierter $\mathrm{CTO}_{\mathrm{NIRA}}$-Lokalisation unter Berücksichtigung von Koronarversorgungstyp und Kollateralstatus von immenser Bedeutung. Dies sollte auch bei der Risiko-Adjustierung in der gesetzlichen Qualitätssicherung Beachtung finden. 


\section{$7 \quad$ Anhang}

\subsection{FITT-STEMI-Dokumentationsbogen}

\section{FiTT-STEMI-Dokumentationsbogen}

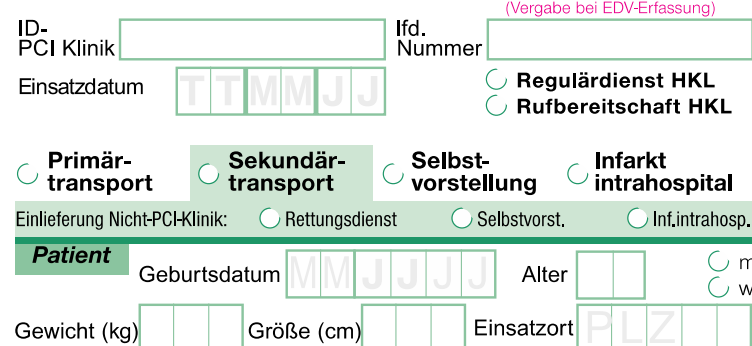

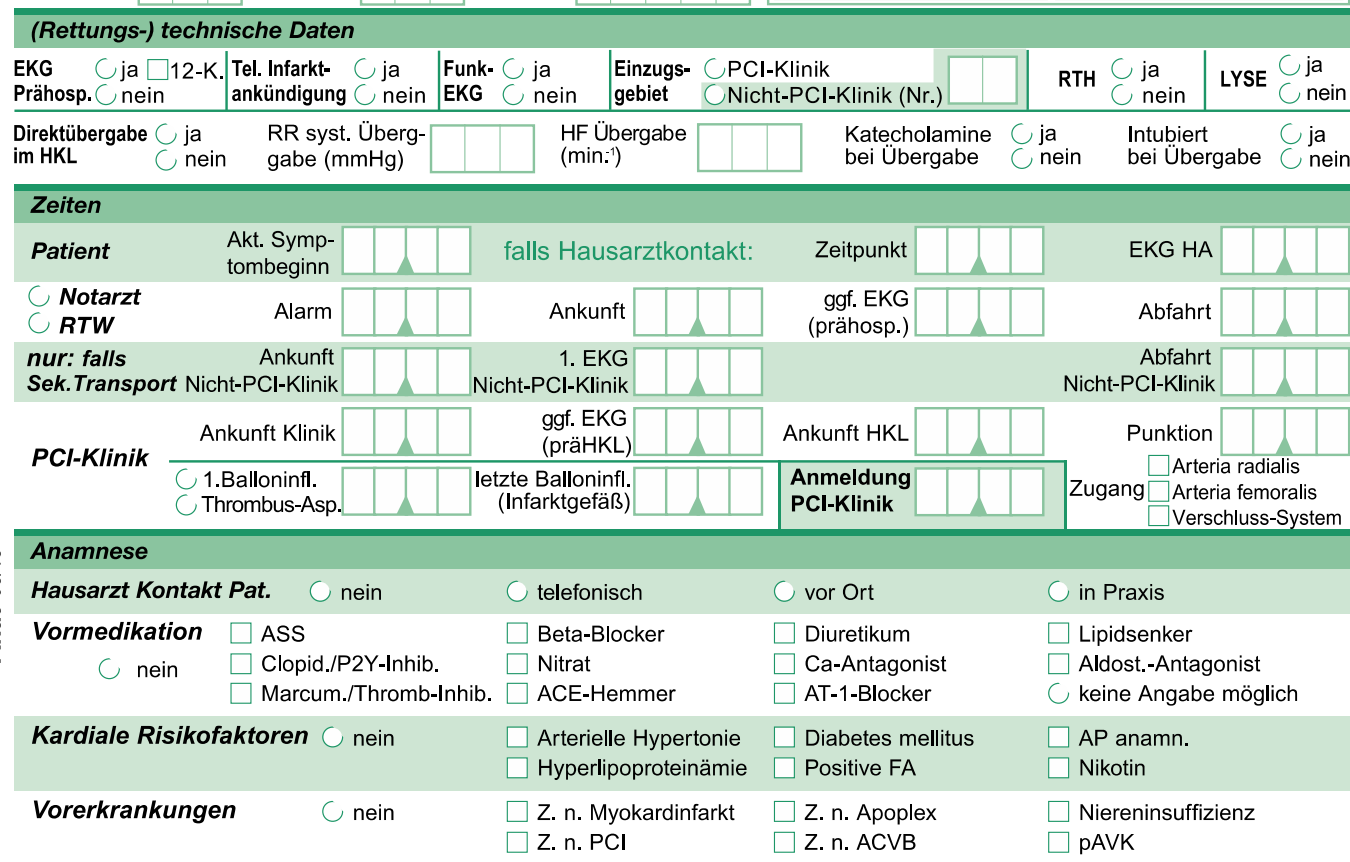

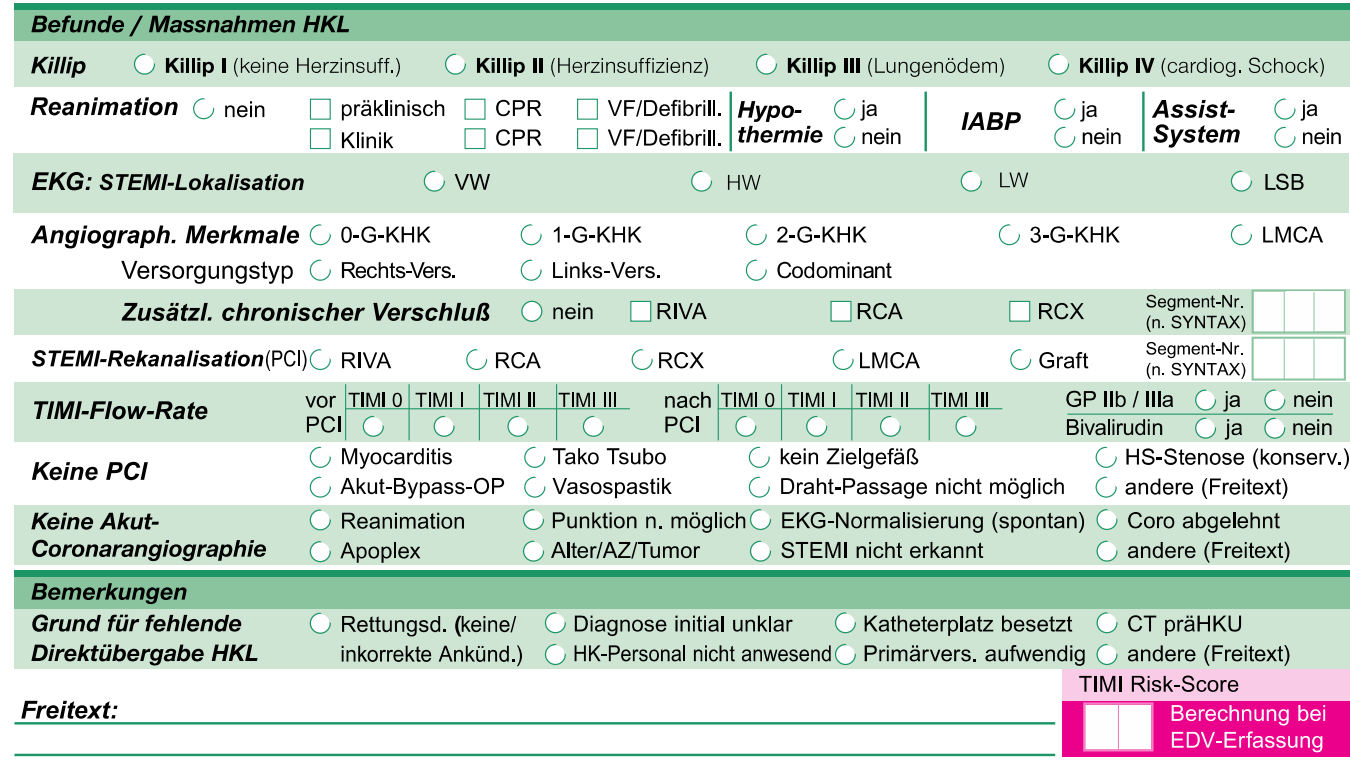

Abbildung 46: Aktueller FITT-STEMI-Dokumentationsbogen (Stand 10/2016, Seite 1/2; (Scholz et al. 2012)) 


\section{FiTT-STEMI-Dokumentationsbogen (Verlauf)}

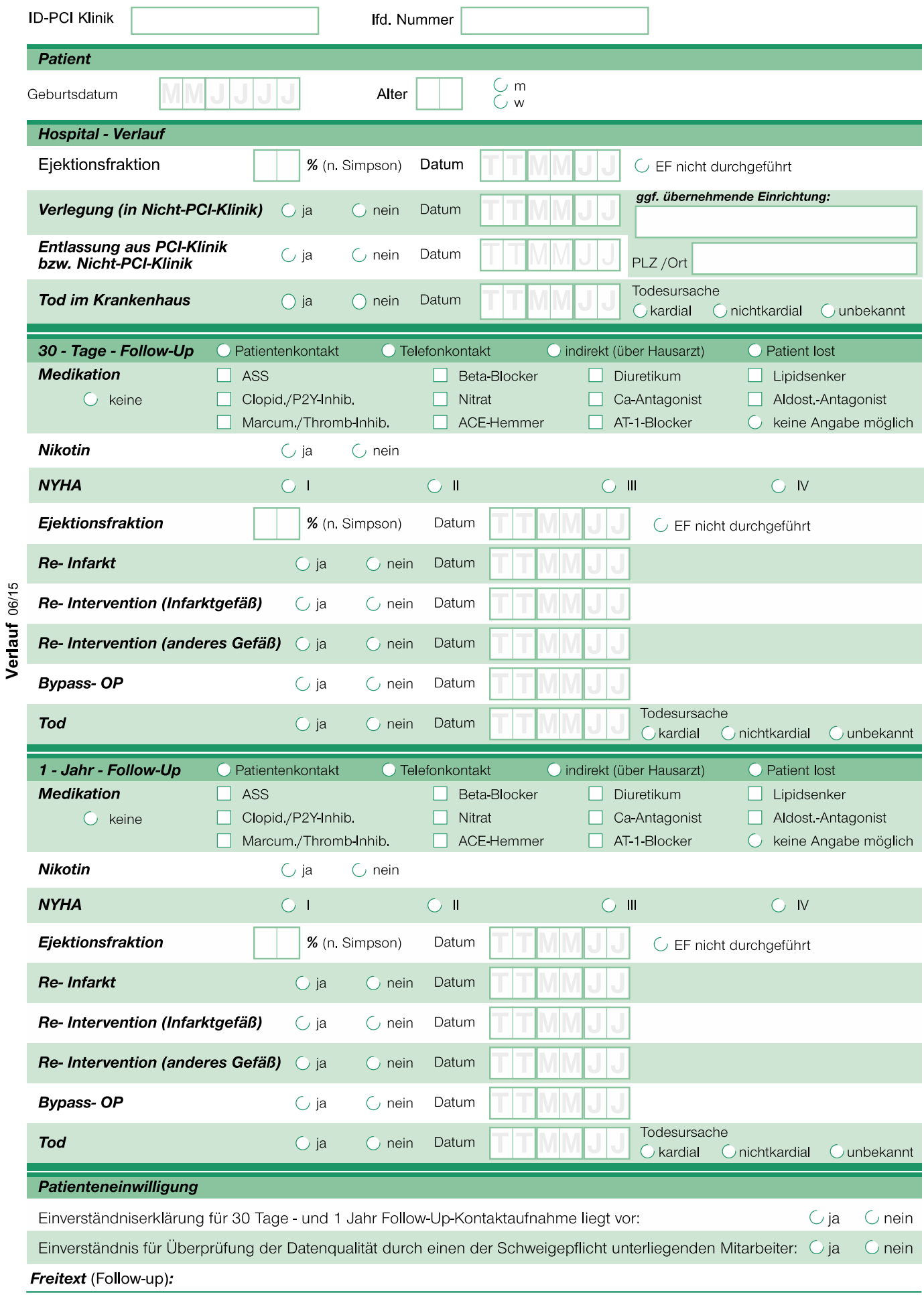

Abbildung 47: Aktueller FITT-STEMI-Dokumentationsbogen (Stand 10/2016, Seite 2/2; (Scholz et al. 2012)) 


\subsection{Angiographischer Beispielbefund}

Die folgenden Abbildungen zeigen beispielhaft einen Koronarangiographiebefund eines Patienten mit MVD $+1 \mathrm{CTO}_{\text {NIRA }}$. Dargestellt ist ein $\mathrm{CTO}_{\text {NIRA }}$ in Segment 1 der RCA mit Brückenkollateralen (Abb. 48) und ein akuter Verschluss in Segment 7 des RIVA (Abb. 49 und 50) bei Rechtsversorgungstyp. Zudem ist eine ausgeprägte interkoronare (septal und epikardial) Kollateralisierung zu erkennen (Rentrop Score 2, CC grade 2; Abb. 51).

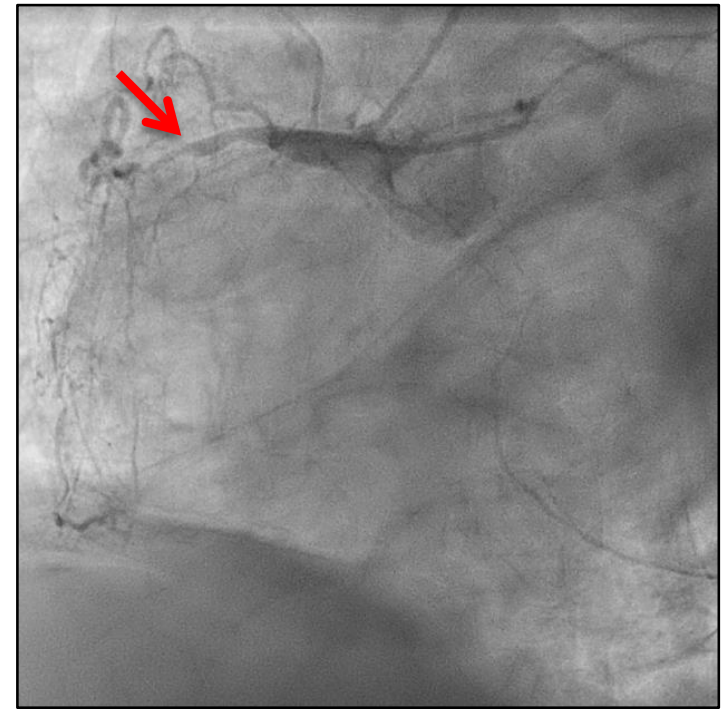

Abbildung 48: CTO in Segment 1 der RCA mit Brückenkollateralen (Projektion: $60^{\circ}$ LAO)

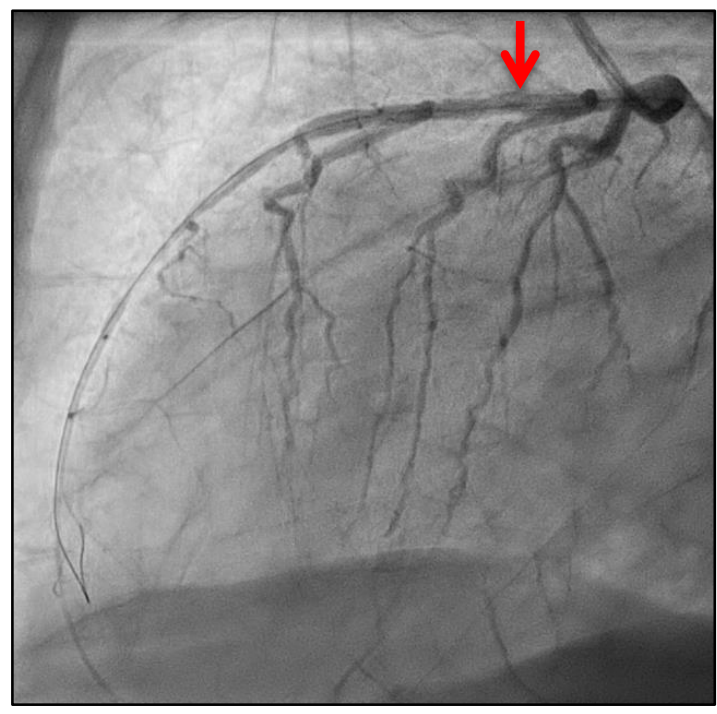

Abbildung 50: Akuter Verschluss in Abbildung 51: Interkoronare Kollaterale Segment 7 des RIVA nach DrahtRekanalisation (Projektion: $90^{\circ} \mathrm{LAO}$ )
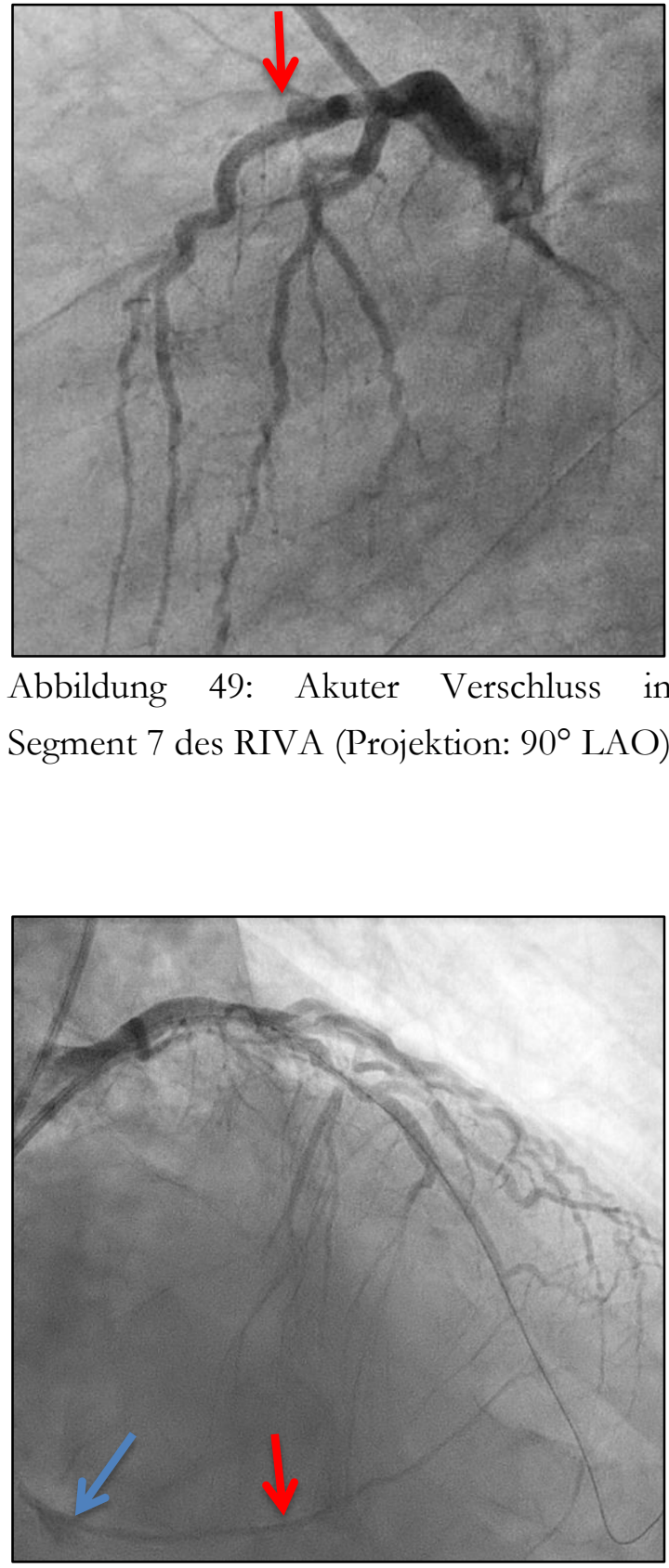

Segment 7 des RIVA (Projektion: 90 LAO) (roter Pfeil) via vormals verschlossenem RIVA nach Rekanalisation mit Darstellung der peripheren RCA (blauer Pfeil, Projektion: $20^{\circ} \mathrm{RAO}$, kranial gekippt) 


\subsection{Basischarakteristika der Gesamtgruppe}

Tabelle 16: Basischarakteristika der Gesamtgruppe und bei Ein- und Mehrgefäß-KHK mit und ohne $\mathrm{CTO}_{\text {NIRA }}$

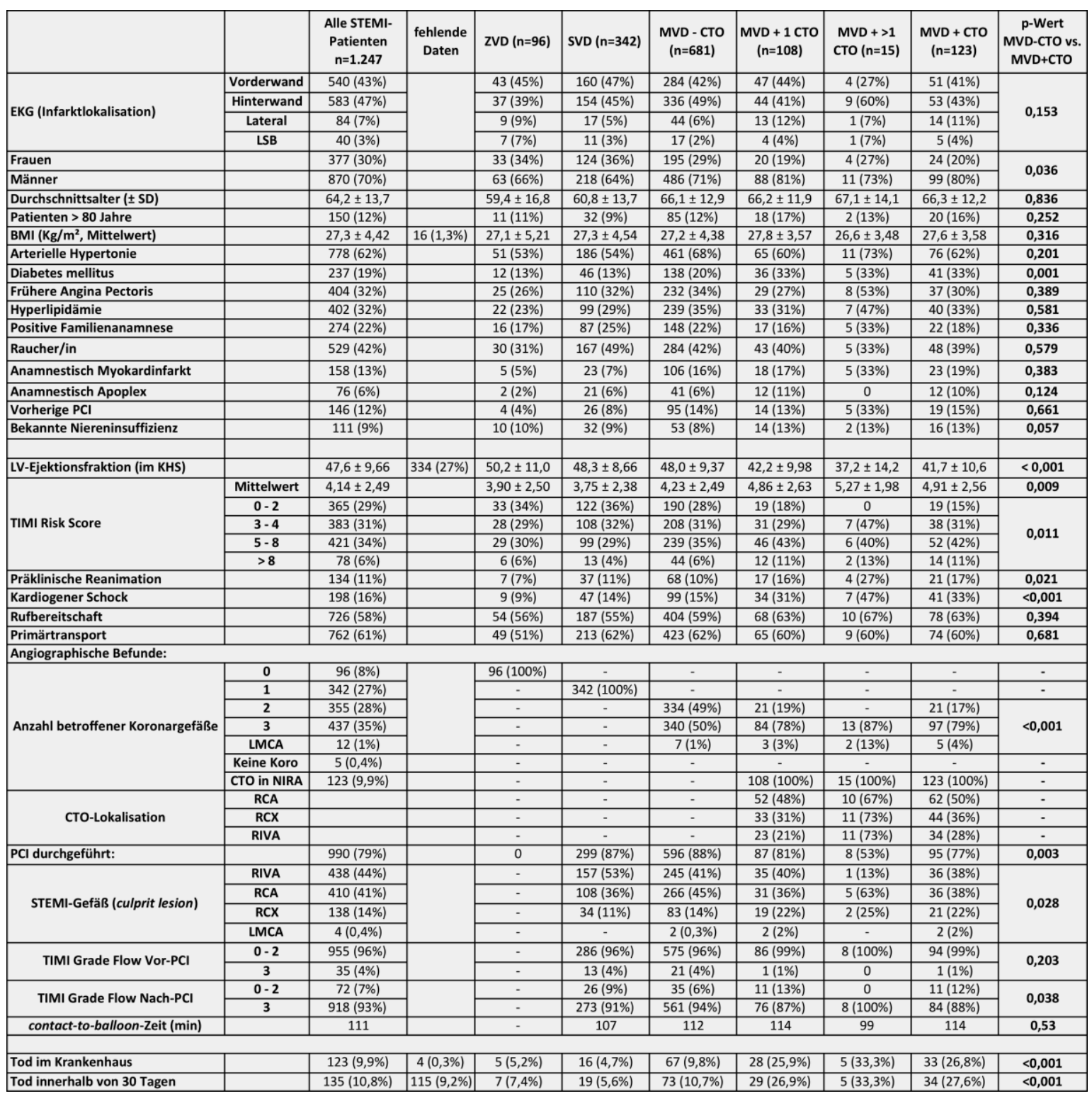




\subsection{Basischarakteristika nach CTO-Gefäßlokalisation}

Tabelle 17: Basischarakteristika aller STEMI-Patienten mit einem $\mathrm{CTO}_{\text {NIRA }}$ in der Gesamtgruppe und nach CTO-Gefäßlokalisation

\begin{tabular}{|c|c|c|c|c|c|c|}
\hline & & $\begin{array}{c}\text { MVD + 1 CTO } \\
(n=108)\end{array}$ & $\begin{array}{l}\text { CTO RCA } \\
(n=52)\end{array}$ & $\begin{array}{l}\text { CTO RIVA } \\
(n=23)\end{array}$ & $\begin{array}{c}\text { CTO RCX } \\
(n=33)\end{array}$ & p-Wert \\
\hline \multirow{4}{*}{ EKG (Infarktlokalisation) } & Vorderwand & $47(44 \%)$ & $30(58 \%)$ & $2(9 \%)$ & $15(45 \%)$ & \multirow{4}{*}{0,002} \\
\hline & Hinterwand & $44(41 \%)$ & $12(23 \%)$ & $16(70 \%)$ & $16(48 \%)$ & \\
\hline & Lateral & $13(12 \%)$ & $8(15 \%)$ & $4(17 \%)$ & $1(3 \%)$ & \\
\hline & LSB & $4(4 \%)$ & $2(4 \%)$ & $1(4 \%)$ & $1(3 \%)$ & \\
\hline Frauen & & $20(19 \%)$ & $5(10 \%)$ & $5(22 \%)$ & $10(30 \%)$ & \multirow{2}{*}{0,052} \\
\hline Männer & & $88(81 \%)$ & $47(90 \%)$ & $18(78 \%)$ & $23(70 \%)$ & \\
\hline Durchschnittsalter \pm Standardabweichung & & $66,2 \pm 11,9$ & $62,9 \pm 11,4$ & $67,1 \pm 11,5$ & $70,9 \pm 11,1$ & 0,002 \\
\hline Patienten $>80$ Jahre & & $18(17 \%)$ & $5(10 \%)$ & $4(17 \%)$ & $9(27 \%)$ & 0,103 \\
\hline BMI (Kg/m², Mittelwert) & & $27,8 \pm 3,57$ & $27,5 \pm 3,6$ & $27,7 \pm 3,7$ & $28,2 \pm 3,39$ & 0,416 \\
\hline Arterielle Hypertonie & & $65(60 \%)$ & $33(63 \%)$ & $13(57 \%)$ & $19(58 \%)$ & 0,796 \\
\hline Diabetes mellitus & & $36(33 \%)$ & $18(35 \%)$ & $5(22 \%)$ & $13(39 \%)$ & 0,372 \\
\hline Frühere Angina Pectoris & & $29(27 \%)$ & $15(29 \%)$ & $6(26 \%)$ & $8(24 \%)$ & 0,892 \\
\hline Hyperlipidämie & & $33(31 \%)$ & $13(25 \%)$ & $9(39 \%)$ & $11(33 \%)$ & 0,433 \\
\hline Positive Familienanamnese & & $17(16 \%)$ & $7(13 \%)$ & $4(17 \%)$ & $6(18 \%)$ & 0,819 \\
\hline Raucher/in & & $43(40 \%)$ & $25(48 \%)$ & $10(43 \%)$ & $8(24 \%)$ & 0,084 \\
\hline Anamnestisch Myokardinfarkt & & $18(17 \%)$ & $10(19 \%)$ & $3(13 \%)$ & $5(15 \%)$ & 0,772 \\
\hline Anamnestisch Apoplex & & $12(11 \%)$ & $4(8 \%)$ & $1(4 \%)$ & $7(21 \%)$ & 0,078 \\
\hline Vorherige $\mathrm{PCl}$ & & $14(13 \%)$ & $6(12 \%)$ & $4(17 \%)$ & $4(12 \%)$ & 0,773 \\
\hline Bekannte Niereninsuffizienz & & $14(13 \%)$ & $8(15 \%)$ & $1(4 \%)$ & $5(15 \%)$ & 0,382 \\
\hline LV-Ejektionsfraktion (im Krankenhaus) & & $42,2 \pm 10,0$ & $42,9 \pm 9,5$ & $42,4 \pm 10,4$ & $40,9 \pm 10.3$ & 0,668 \\
\hline \multirow{5}{*}{ TIMI Risk Score } & Mittelwert & $4,86 \pm 2,63$ & $4,9 \pm 2,59$ & $4,61 \pm 2,43$ & $4,97 \pm 2.8$ & 0,843 \\
\hline & $0-2$ & $19(18 \%)$ & $8(15 \%)$ & $5(22 \%)$ & $6(18 \%)$ & \multirow{4}{*}{0,628} \\
\hline & $3-4$ & $31(29 \%)$ & $17(33 \%)$ & $8(35 \%)$ & $6(18 \%)$ & \\
\hline & $5-8$ & $46(43 \%)$ & $20(38 \%)$ & $9(39 \%)$ & $17(52 \%)$ & \\
\hline & $>8$ & $12(11 \%)$ & $7(13 \%)$ & $1(4 \%)$ & $4(12 \%)$ & \\
\hline Präklinische Reanimation & & $17(16 \%)$ & $8(15 \%)$ & $3(13 \%)$ & $6(18 \%)$ & 0,87 \\
\hline Kardiogener Schock & & $34(31 \%)$ & $18(35 \%)$ & $7(30 \%)$ & $9(27 \%)$ & 0,771 \\
\hline Rufbereitschaft & & $68(63 \%)$ & $36(69 \%)$ & $9(39 \%)$ & $23(70 \%)$ & 0,028 \\
\hline Primärtransport & & $65(60 \%)$ & $30(58 \%)$ & $15(65 \%)$ & $20(61 \%)$ & 0,827 \\
\hline Angiographische Befunde: & & & & & & \\
\hline \multirow{5}{*}{ Anzahl betroffener Koronargefäße } & 0 & - & - & - & - & - \\
\hline & 1 & - & - & - & - & - \\
\hline & 2 & $21(19 \%)$ & $10(19 \%)$ & $5(22 \%)$ & $6(18 \%)$ & \multirow{3}{*}{0,364} \\
\hline & 3 & $84(78 \%)$ & $41(79 \%)$ & $16(70 \%)$ & $27(82 \%)$ & \\
\hline & LMCA & $3(3 \%)$ & $1(2 \%)$ & $2(9 \%)$ & 0 & \\
\hline \multirow{3}{*}{ Сто-Lokalisation } & RCA & $52(48 \%)$ & & & & - \\
\hline & RCX & $33(31 \%)$ & & & & - \\
\hline & RIVA & $23(21 \%)$ & & & & - \\
\hline Versoraungstun & Rechts & $97(90 \%)$ & $42(81 \%)$ & $22(96 \%)$ & $33(100 \%)$ & \multirow{2}{*}{0,01} \\
\hline Versorgungstyp & Links & $11(10 \%)$ & $10(19 \%)$ & $1(4 \%)$ & 0 & \\
\hline Kollateralen & & $92(85 \%)$ & $47(90 \%)$ & $20(87 \%)$ & $25(76 \%)$ & 0,174 \\
\hline $\mathrm{PCl}$ durchgeführt: & & $87(81 \%)$ & $39(75 \%)$ & $21(91 \%)$ & $27(82 \%)$ & 0,252 \\
\hline \multirow{4}{*}{ STEMI-Gefäß (culprit lesion) } & RIVA & $35(40 \%)$ & $23(59 \%)$ & - & $12(44 \%)$ & \multirow{4}{*}{$<0,001$} \\
\hline & RCA & $31(36 \%)$ & - & $16(76 \%)$ & $15(56 \%)$ & \\
\hline & RCX & $19(22 \%)$ & $15(38 \%)$ & $4(19 \%)$ & - & \\
\hline & LMCA & $2(2 \%)$ & $1(3 \%)$ & $1(5 \%)$ & 0 & \\
\hline \multirow{2}{*}{ TIMI Grade Flow Vor-PCI } & $0-2$ & $86(99 \%)$ & $38(97 \%)$ & $21(100 \%)$ & $27(100 \%)$ & \multirow{2}{*}{0,537} \\
\hline & 3 & $1(1 \%)$ & $1(3 \%)$ & 0 & 0 & \\
\hline \multirow{2}{*}{ TIMI Grade Flow Nach-PCI } & $0-2$ & $11(13 \%)$ & $6(15 \%)$ & 0 & $5(19 \%)$ & \multirow{2}{*}{0,126} \\
\hline & 3 & $76(87 \%)$ & $33(85 \%)$ & $21(100 \%)$ & $22(81 \%)$ & \\
\hline Tod im Krankenhaus & & $28(25,9 \%)$ & $16(30,8 \%)$ & $5(21,7 \%)$ & $7(21,2 \%)$ & 0,541 \\
\hline Tod innerhalb von 30 Tagen & & $29(26,9 \%)$ & $17(32,7 \%)$ & $5(21,7 \%)$ & $7(21,2 \%)$ & 0,418 \\
\hline
\end{tabular}


Tabelle 18: Basischarakteristika aller STEMI-Patienten mit einem $\mathrm{CTO}_{\text {NIRA }}$ : Lokalisation in Segment 1 der RCA bei Rechtsversorgungstyp vs. alle anderen STEMI-Patienten mit einem $\mathrm{CTO}_{\text {NIRA }}$

\begin{tabular}{|c|c|c|c|c|c|}
\hline & & $\begin{array}{c}\text { MVD + } 1 \text { Сто } \\
(n=108)\end{array}$ & $\begin{array}{c}\text { CTO RCA } \\
\text { Segment } 1 \text { bei } \\
\text { Rechtsver- } \\
\text { sorgungstyp } \\
(n=26)\end{array}$ & $\begin{array}{c}\text { Alle anderen } \\
\text { CTO } \\
\text { Lokalisationen } \\
(n=82)\end{array}$ & p-Wert \\
\hline \multirow{4}{*}{ EKG (Infarktlokalisation) } & Vorderwand & $47(44 \%)$ & $15(58 \%)$ & $32(39 \%)$ & \multirow{4}{*}{0,074} \\
\hline & Hinterwand & $44(41 \%)$ & $5(19 \%)$ & $39(48 \%)$ & \\
\hline & Lateral & $13(12 \%)$ & $5(19 \%)$ & $8(10 \%)$ & \\
\hline & LSB & $4(4 \%)$ & $1(4 \%)$ & $3(4 \%)$ & \\
\hline Frauen & & $20(19 \%)$ & $2(8 \%)$ & $18(22 \%)$ & \multirow{2}{*}{0,103} \\
\hline Männer & & $88(81 \%)$ & $24(92 \%)$ & $64(78 \%)$ & \\
\hline Alter \pm Standardabweichung & Mittelwert & $66,2 \pm 11,9$ & $60,9 \pm 11,2$ & $67,9 \pm 11,6$ & 0,009 \\
\hline Patienten > 80 Jahre & & $18(17 \%)$ & $3(12 \%)$ & $15(18 \%)$ & 0,421 \\
\hline BMI $\left(\mathrm{Kg} / \mathrm{m}^{2}\right)$ & Mittelwert & $27,8 \pm 3,57$ & $28,3 \pm 3,52$ & $27,6 \pm 3,57$ & 0,418 \\
\hline Arterielle Hypertonie & & $65(60 \%)$ & $15(58 \%)$ & $50(61 \%)$ & 0,766 \\
\hline Diabetes mellitus & & $36(33 \%)$ & $9(35 \%)$ & $27(33 \%)$ & 0,874 \\
\hline Frühere Angina Pectoris & & $29(27 \%)$ & $8(31 \%)$ & $21(26 \%)$ & 0,605 \\
\hline Hyperlipidämie & & $33(31 \%)$ & $6(23 \%)$ & $27(33 \%)$ & 0,342 \\
\hline Positive Familienanamnese & & $17(16 \%)$ & $3(12 \%)$ & $14(17 \%)$ & 0,5 \\
\hline Raucher/in & & $43(40 \%)$ & $12(46 \%)$ & $31(38 \%)$ & 0,449 \\
\hline Anamnestisch Myokardinfarkt & & $18(17 \%)$ & $4(15 \%)$ & $14(17 \%)$ & 0,84 \\
\hline Anamnestisch Apoplex & & $12(11 \%)$ & $3(12 \%)$ & $9(11 \%)$ & 0,937 \\
\hline Vorherige $\mathrm{PCl}$ & & $14(13 \%)$ & $3(12 \%)$ & $11(13 \%)$ & 0,804 \\
\hline Bekannte Niereninsuffizienz & & $14(13 \%)$ & $5(19 \%)$ & $9(11 \%)$ & 0,275 \\
\hline LV-Ejektionsfraktion (im Krankenhaus) & Mittelwert & $42,2 \pm 10,0$ & $43,5 \pm 8,4$ & $41,8 \pm 10,4$ & 0,792 \\
\hline \multirow{5}{*}{ TIMI Risk Score } & Mittelwert & $4,86 \pm 2,63$ & $4,69 \pm 1,86$ & $4,91 \pm 2,82$ & 0,966 \\
\hline & $0-2$ & $19(18 \%)$ & $3(12 \%)$ & $16(20 \%)$ & \multirow{4}{*}{0,362} \\
\hline & $3-4$ & $31(29 \%)$ & $9(35 \%)$ & $22(27 \%)$ & \\
\hline & $5-8$ & $46(43 \%)$ & $13(50 \%)$ & $33(40 \%)$ & \\
\hline & $>8$ & $12(11 \%)$ & $1(4 \%)$ & $11(13 \%)$ & \\
\hline Präklinische Reanimation & & $17(16 \%)$ & $6(23 \%)$ & $11(13 \%)$ & 0,238 \\
\hline Kardiogener Schock & & $34(31 \%)$ & $12(46 \%)$ & $22(27 \%)$ & 0,065 \\
\hline Rufbereitschaft & & $68(63 \%)$ & $15(58 \%)$ & $53(65 \%)$ & 0,523 \\
\hline Primärtransport & & $65(60 \%)$ & $16(62 \%)$ & $49(60 \%)$ & 0,871 \\
\hline \multicolumn{6}{|l|}{ Angiographische Befunde: } \\
\hline \multirow{5}{*}{ Anzahl betroffener Koronargefäße } & 0 & - & - & - & \multirow{5}{*}{0,215} \\
\hline & 1 & - & - & - & \\
\hline & 2 & $21(19 \%)$ & $2(8 \%)$ & $19(23 \%)$ & \\
\hline & 3 & $84(78 \%)$ & $23(88 \%)$ & $61(74 \%)$ & \\
\hline & LMCA & $3(3 \%)$ & $1(4 \%)$ & $2(2 \%)$ & \\
\hline \multirow{3}{*}{ Сто-Lokalisation } & RCA & $52(48 \%)$ & $26(100 \%)$ & $26(32 \%)$ & \multirow{3}{*}{ - } \\
\hline & RCX & $33(31 \%)$ & 0 & $33(40 \%)$ & \\
\hline & RIVA & $23(21 \%)$ & 0 & $23(28 \%)$ & \\
\hline \multirow{2}{*}{ Versorgungstyp } & Rechts & $97(90 \%)$ & $26(100 \%)$ & $71(87 \%)$ & \multirow[b]{2}{*}{ - } \\
\hline & Links & $11(10 \%)$ & 0 & $11(13 \%)$ & \\
\hline Kollateralen & & $92(85 \%)$ & $24(92 \%)$ & $68(83 \%)$ & 0,241 \\
\hline PCI durchgeführt: & & $87(81 \%)$ & $18(69 \%)$ & $69(84 \%)$ & 0,094 \\
\hline \multirow{4}{*}{ STEMI-Gefäß (culprit lesion) } & RIVA & $35(40 \%)$ & $11(61 \%)$ & $24(35 \%)$ & \multirow{4}{*}{0,005} \\
\hline & RCA & $31(36 \%)$ & - & $31(45 \%)$ & \\
\hline & RCX & $19(22 \%)$ & $6(33 \%)$ & $13(19 \%)$ & \\
\hline & LMCA & $2(2 \%)$ & $1(6 \%)$ & $1(1 \%)$ & \\
\hline \multirow{2}{*}{ TIMI Grade Flow Vor-PCI } & $0-2$ & $86(99 \%)$ & $18(100 \%)$ & $68(99 \%)$ & \multirow{2}{*}{0,607} \\
\hline & 3 & $1(1 \%)$ & 0 & $1(1 \%)$ & \\
\hline \multirow{2}{*}{ TIMI Grade Flow Nach-PCI } & $0-2$ & $11(13 \%)$ & $4(22 \%)$ & $7(10 \%)$ & \multirow{2}{*}{0,17} \\
\hline & 3 & $76(87 \%)$ & $14(78 \%)$ & $62(90 \%)$ & \\
\hline Tod im Krankenhaus & & $28(25,9 \%)$ & $11(42,3 \%)$ & $17(20,7 \%)$ & 0,029 \\
\hline Tod innerhalb von 30 Tagen & & $29(26,9 \%)$ & $12(46,2 \%)$ & $17(20,7 \%)$ & 0,011 \\
\hline
\end{tabular}


Tabelle 19: Endgültiges Ergebnis der backward stepwise-Modell-Konstruktion nach 19 Schritten; Übersicht über alle in diesem multivariaten Modell berücksichtigten Variablen

\begin{tabular}{|c|c|c|c|c|c|}
\hline Variable & Wald stat. & Wald $\mathrm{p}$ & Score stat. & Score $\mathrm{p}$ & Var. Status \\
\hline Raucher/in & 4,153897 & 0,041539 & & & In \\
\hline Alter & 4,016391 & 0,045060 & & & In \\
\hline Z. n. PCI & 6,205850 & 0,012733 & & & In \\
\hline Kardiogener Schock & 21,334431 & 0,000004 & & & In \\
\hline $\begin{array}{l}\text { CTO in Segment } 1 \text { der RCA bei } \\
\text { Rechtsversorgungstyp }\end{array}$ & 4,580534 & 0,032337 & & & In \\
\hline Arterielle Hypertonie & & & 2,788559 & 0,094940 & Out \\
\hline Präklinische Reanimation & & & 2,634740 & 0,104549 & Out \\
\hline EKG Infarktlokalisation: Hinterwand & & & 1,069899 & 0,300968 & Out \\
\hline Versorgungstyp & & & 1,758971 & 0,184753 & Out \\
\hline Q-Zacke & & & 0,287379 & 0,591905 & Out \\
\hline Niereninsuffizienz & & & 0,414569 & 0,519659 & Out \\
\hline Anamnestisch Myokardinfarkt & & & 0,381290 & 0,536914 & Out \\
\hline Hyperlipidämie & & & 0,272860 & 0,601420 & Out \\
\hline Frühere Angina Pectoris & & & 0,462779 & 0,496328 & Out \\
\hline Positive Familienanamnese & & & 0,022815 & 0,879938 & Out \\
\hline EKG Infarktlokalisation: Lateralwand & & & 0,004641 & 0,945685 & Out \\
\hline Geschlecht & & & 1,397556 & 0,237133 & Out \\
\hline 3-Gefäß-KHK und Hauptstammstenose & & & 0,048383 & 0,825901 & Out \\
\hline Kollateralen & & & 0,381867 & 0,536606 & Out \\
\hline Body Mass Index (BMI) & & & 0,007442 & 0,931256 & Out \\
\hline PCI durchgeführt & & & 0,110077 & 0,740057 & Out \\
\hline Diabetes mellitus & & & 0,601463 & 0,438020 & Out \\
\hline EKG Infarktlokalisation: Vorderwand & & & 0,780401 & 0,377019 & Out \\
\hline
\end{tabular}




\section{$8 \quad$ Literaturverzeichnis}

Abbott JD, Kip KE, Vlachos HA, Sawhney N, Srinivas VS, Jacobs AK, Holmes DR, Williams DO (2006): Recent trends in the percutaneous treatment of chronic total coronary occlusions. Am J Cardiol 97, 1691-1696

Alpert J, Thygesen K (2000): Myocardial infarction redefined - A consensus document of The Joint European Society of Cardiology/American College of Cardiology Committee for the redefinition of myocardial infarction. Eur Heart J 21, 1502-1513

Andersen HR, Nielsen TT, Rasmussen K, Thuesen L, Kelbaek H, Thayssen P, Abildgaard U, Pedersen F, Madsen JK, Grande P, et al. (2003): A comparison of coronary angioplasty with fibrinolytic therapy in acute myocardial infarction. N Engl J Med $\underline{349}, 733-742$

Angelini P, Velasco JA, Flamm S (2002): Coronary anomalies: incidence, pathophysiology, and clinical relevance. Circulation $\underline{105}, 2449-2454$

Antoniucci D, Valenti R, Moschi G, Migliorini A, Trapani M, Santoro GM, Bolognese L, Cerisano G, Buonamici P, Dovellini EV (2002): Relation between preintervention angiographic evidence of coronary collateral circulation and clinical and angiographic outcomes after primary angioplasty or stenting for acute myocardial infarction. Am J Cardiol $\underline{89}, 121-125$

AQUA - Institut für angewandte Qualitätsförderung und Forschung im Gesundheitswesen GmbH. Qualitätsreport 2013. Göttingen 2014

Ariza-Solé A, Teruel L, di Marco A, Lorente V, Sánchez-Salado JC, Sánchez-Elvira G, Romaguera R, Gómez-Lara J, Gómez-Hospital JA, Cequier A (2014): Prognostic impact of chronic total occlusion in a nonculprit artery in patients with acute myocardial infarction undergoing primary angioplasty. Rev Esp Cardiol Engl Ed 67, 359-366

Austen WG, Edwards JE, Frye RL, Gensini GG, Gott VL, Griffith LS, McGoon DC, Murphy ML, Roe BB (1975): A reporting system on patients evaluated for coronary artery disease. Report of the Ad Hoc Committee for Grading of Coronary Artery Disease, Council on Cardiovascular Surgery, American Heart Association. Circulation 1ㅗ, 5-40

Baer FM, Rosenkranz S: Koronare Herzkrankheit und akutes Koronarsyndrom. In: Erdmann E (Hrsg.): Klinische Kardiologie: Krankheiten des Herzens, des Kreislaufs und der herznahen Gefäße. 8. Auflage; Springer, Berlin, Heidelberg 2011, 13-72

Baks T, van Geuns R-J, Duncker DJ, Cademartiri F, Mollet NR, Krestin GP, Serruys PW, de Feyter PJ (2006): Prediction of left ventricular function after drug-eluting stent implantation for chronic total coronary occlusions. J Am Coll Cardiol 47, 721-725 
Bataille Y, Déry J-P, Larose É, Déry U, Costerousse O, Rodés-Cabau J, Gleeton O, Proulx G, Abdelaal E, Machaalany J, et al. (2012a): Deadly association of cardiogenic shock and chronic total occlusion in acute ST-elevation myocardial infarction. Am Heart J 164,509 515

Bataille Y, Déry J-P, Larose E, Déry U, Costerousse O, Rodés-Cabau J, Rinfret S, De Larochellière R, Abdelaal E, Machaalany J, et al. (2012b): Prevalence, predictors and clinical impact of unique and multiple chronic total occlusion in non-infarct-related artery in patients presenting with ST-elevation myocardial infarction. Heart $\underline{98}$, 1732-1737

Bataille Y, Déry J-P, Larose É, Abdelaal E, Machaalany J, Rodés-Cabau J, Rinfret S, Déry U, Costerousse O, Roy L, Betrand OF (2013): Incidence and clinical impact of concurrent chronic total occlusion according to gender in ST-elevation myocardial infarction. Catheter Cardiovasc Interv $\underline{82}, 19-26$

Bazzocchi G, Romagnoli A, Sperandio M, Simonetti G (2011): Evaluation with 64-slice CT of the prevalence of coronary artery variants and congenital anomalies: a retrospective study of 3,236 patients. Radiol Med (Torino) 116, 675-689

Berger PB, Ellis SG, Holmes DR, Granger CB, Criger DA, Betriu A, Topol EJ, Califf RM (1999): Relationship between delay in performing direct coronary angioplasty and early clinical outcome in patients with acute myocardial infarction: results from the global use of strategies to open occluded arteries in Acute Coronary Syndromes (GUSTO-IIb) trial. Circulation 100, 14-20

Bernard R, Corday E, Eliasch H, Gonin A, Hiait R, Nikolaeva LF, Oakley CM, Oliver MF, Pisa Z, Puddu V, et al. (1979): Nomenclature and criteria for diagnosis of ischemic heart disease. Report of the Joint International Society and Federation of Cardiology/World Health Organization task force on standardization of clinical nomenclature. Circulation $\underline{59}$, 607-609

Betriu A, Phillips H, Ellis S, Topol E, Califf R, Van der Werf F, Ardissino D, Armstrong PW, Aylward P, Bates E, et al. (1997): A Clinical Trial Comparing Primary Coronary Angioplasty with Tissue Plasminogen Activator for Acute Myocardial Infarction. N Engl J Med $\underline{336}, 1621-1628$

Bültmann BD, Mackensen-Haen S, Kirkpatrick CJ, Baba HA: Herz. In: Böcker W, Denk H, Heitz PU, Moch H (Hrsg.): Pathologie. 4. Auflage; Urban \& Fischer Verlag/Elsevier GmbH, München 2008, 450-494 
Carlino M, Magri CJ, Uretsky BF, Brilakis ES, Walsh S, Spratt JC, Hanratty C, Grantham JA, Rinfret S, Thompson CA, et al. (2015): Treatment of the chronic total occlusion: a call to action for the interventional community. Catheter Cardiovasc Interv $\underline{85}, 771-778$

Cavender MA, Milford-Beland S, Roe MT, Peterson ED, Weintraub WS, Rao SV (2009): Prevalence, predictors, and in-hospital outcomes of non-infarct artery intervention during primary percutaneous coronary intervention for ST-segment elevation myocardial infarction (from the National Cardiovascular Data Registry). Am J Cardiol 104, 507-513

Chesebro JH, Knatterud G, Roberts R, Borer J, Cohen LS, Dalen J, Dodge HT, Francis CK, Hillis D, Ludbrook P (1987): Thrombolysis in Myocardial Infarction (TIMI) Trial, Phase I: A comparison between intravenous tissue plasminogen activator and intravenous streptokinase. Clinical findings through hospital discharge. Circulation $\underline{76}$, 142-154

Christofferson RD, Lehmann KG, Martin GV, Every N, Caldwell JH, Kapadia SR (2005): Effect of chronic total coronary occlusion on treatment strategy. Am J Cardiol 모, 10881091

Claessen BE, van der Schaaf RJ, Verouden NJ, Stegenga NK, Engstrom AE, Sjauw KD, Kikkert WJ, Vis MM, Baan J, Koch KT, et al. (2009): Evaluation of the effect of a concurrent chronic total occlusion on long-term mortality and left ventricular function in patients after primary percutaneous coronary intervention. JACC Cardiovasc Interv $\underline{2}$, 1128-1134

Claessen BE, Hoebers LP, van der Schaaf RJ, Kikkert WJ, Engstrom AE, Vis MM, Baan J, Koch KT, Meuwissen M, van Royen N, et al. (2010): Prevalence and impact of a chronic total occlusion in a non-infarct-related artery on long-term mortality in diabetic patients with ST elevation myocardial infarction. Heart $\underline{96}, 1968-1972$

Claessen BE, Dangas GD, Weisz G, Witzenbichler B, Guagliumi G, Möckel M, Brener SJ, Xu K, Henriques JPS, Mehran R, Stone GW (2012): Prognostic impact of a chronic total occlusion in a non-infarct-related artery in patients with ST-segment elevation myocardial infarction: 3-year results from the HORIZONS-AMI trial. Eur Heart J $\underline{33}$, 768-775

Claessen BE, Dangas GD, Godino C, Henriques JPS, Leon MB, Park S-J, Stone GW, Moses JW, Colombo A, Mehran R (2013): Impact of target vessel on long-term survival after percutaneous coronary intervention for chronic total occlusions. Catheter Cardiovasc Interv $\underline{82}, 76-82$

Daves ML (1970): Cardiac roentgenology. Tho loop and circle approach. Radiology 95, $157-160$ 
Davies MJ (2000): The pathophysiology of acute coronary syndromes. Heart $\underline{83}$, 361-366

De Mulder M, Gitt A, van Domburg R, Hochadel M, Seabra-Gomes R, Serruys PW, Silber S, Weidinger F, Wijns W, Zeymer U, et al. (2011): EuroHeart score for the evaluation of inhospital mortality in patients undergoing percutaneous coronary intervention. Eur Heart J $\underline{32}, 1398-1408$

Di Mario C, Werner GS, Sianos G, Galassi AR, Büttner J, Dudek D, Chevalier B, Lefevre T, Schofer J, Koolen J, et al. (2007): European perspective in the recanalisation of Chronic Total Occlusions (CTO): consensus document from the EuroCTO Club. EuroIntervention $\underline{3}, 30-43$

Dill T, Brandt R, Hamm CW: Akutes Koronarsyndrom: Instabile Angina pectoris und Myokardinfarkt. In: Baenkler H-W, Arastéh K, Bieber C, Brandt R, Chatterjee TT (Hrsg.): Duale Reihe Innere Medizin. 2. Auflage; Thieme, Stuttgart 2013, 58-72

Drenckhahn D: Anatomie, Makroskopische Anatomie, Embryologie und Histologie des Menschen. Band 2: Herz-Kreislauf-System, Lymphatisches System, Endokrine Drüsen, Nervensystem, Sinnesorgane, Haut. 16. Auflage; Urban \& Fischer Verlag/Elsevier GmbH, München, Jena 2004

Elsman P, van 't Hof AWJ, de Boer MJ, Hoorntje JCA, Suryapranata H, Dambrink JHE, Zijlstra F (2004): Role of collateral circulation in the acute phase of ST-segment-elevation myocardial infarction treated with primary coronary intervention. Eur Heart J 25, 854-858

Emond M, Mock MB, Davis KB, Fisher LD, Holmes DR, Chaitman BR, Kaiser GC, Alderman E, Killip T (1994): Long-term survival of medically treated patients in the Coronary Artery Surgery Study (CASS) Registry. Circulation 90, 2645-2657

Falk E, Shah PK, Fuster V (1995): Coronary plaque disruption. Circulation 92, 657-671

Fefer P, Knudtson ML, Cheema AN, Galbraith PD, Osherov AB, Yalonetsky S, Gannot S, Samuel M, Weisbrod M, Bierstone D, et al. (2012): Current perspectives on coronary chronic total occlusions: the Canadian Multicenter Chronic Total Occlusions Registry. J Am Coll Cardiol 59, 991-997

Fox KAA, Dabbous OH, Goldberg RJ, Pieper KS, Eagle KA, Van de Werf F, Avezum A, Goodman SG, Flather MD, Anderson FA, Granger CB (2006): Prediction of risk of death and myocardial infarction in the six months after presentation with acute coronary syndrome: prospective multinational observational study (GRACE). BMJ 333, 1091

Fujii K, Ochiai M, Mintz GS, Kan Y, Awano K, Masutani M, Ashida K, Ohyanagi M, Ichikawa S, Ura S, et al. (2006): Procedural implications of intravascular ultrasound morphologic features of chronic total coronary occlusions. Am J Cardiol 구, 1455-1462 
Garg S, Sarno G, Serruys PW, Rodriguez AE, Bolognese L, Anselmi M, De Cesare N, Colangelo S, Moreno R, Gambetti S, et al. (2011): Prediction of 1-year clinical outcomes using the SYNTAX score in patients with acute ST-segment elevation myocardial infarction undergoing primary percutaneous coronary intervention: a substudy of the STRATEGY (Single High-Dose Bolus Tirofiban and Sirolimus-Eluting Stent Versus Abciximab and Bare-Metal Stent in Acute Myocardial Infarction) and MULTISTRATEGY (Multicenter Evaluation of Single High-Dose Bolus Tirofiban Versus Abciximab With Sirolimus-Eluting Stent or Bare-Metal Stent in Acute Myocardial Infarction Study) trials. JACC Cardiovasc Interv $\underline{4}, 66-75$

Gensini GG (1983): A more meaningful scoring system for determining the severity of coronary heart disease. Am J Cardiol $\underline{51}, 606$

Gersh BJ, Stone GW, White HD, Holmes DR (2005): Pharmacological facilitation of primary percutaneous coronary intervention for acute myocardial infarction: is the slope of the curve the shape of the future? JAMA $\underline{293}$, 979-986

Goldberg A, Southern DA, Galbraith PD, Traboulsi M, Knudtson ML, Ghali WA (2007): Coronary dominance and prognosis of patients with acute coronary syndrome. Am Heart J $\underline{154}, 1116-1122$

Gould K (1989): Coronary steal. Is it clinically important? Chest $\underline{96}, 227-228$

Grines C, Patel A, Zijlstra F, Weaver WD, Granger C, Simes RJ (2003): Primary coronary angioplasty compared with intravenous thrombolytic therapy for acute myocardial infarction: six-month follow up and analysis of individual patient data from randomized trials. Am Heart J $\underline{145}, 47-57$

Grines CL, Browne KF, Marco J, Rothbaum D, Stone GW, O'Keefe J, Overlie P, Donohue B, Chelliah N, Timmis GC (1993): A comparison of immediate angioplasty with thrombolytic therapy for acute myocardial infarction. The Primary Angioplasty in Myocardial Infarction Study Group. N Engl J Med $\underline{328}$, 673-679

Grines CL, Cox DA, Stone GW, Garcia E, Mattos LA, Giambartolomei A, Brodie BR, Madonna O, Eijgelshoven M, Lansky AJ, et al. (1999): Coronary angioplasty with or without stent implantation for acute myocardial infarction. Stent Primary Angioplasty in Myocardial Infarction Study Group. N Engl J Med 341, 1949-1956 
Grines CL, Westerhausen DR, Grines LL, Hanlon JT, Logemann TL, Niemela M, Weaver WD, Graham M, Boura J, O’Neill WW, Balestrini C (2002): A randomized trial of transfer for primary angioplasty versus on-site thrombolysis in patients with high-risk myocardial infarction: the Air Primary Angioplasty in Myocardial Infarction study. J Am Coll Cardiol $\underline{39}, 1713-1719$

Guo J, Maehara A, Mintz GS, Ashida K, Pu J, Shang Y, Leon MB, Stone GW, Moses JW, Ochiai M (2013): A virtual histology intravascular ultrasound analysis of coronary chronic total occlusions. Catheter Cardiovasc Interv $\underline{81}, 464-470$

Halkin A, Stone GW, Dixon SR, Grines CL, Tcheng JE, Cox DA, Garcia E, Brodie B, Stuckey TD, Mehran R, Lansky AJ (2005a): Impact and determinants of left ventricular function in patients undergoing primary percutaneous coronary intervention in acute myocardial infarction. Am J Cardiol $\underline{96}, 325-331$

Halkin A, Singh M, Nikolsky E, Grines CL, Tcheng JE, Garcia E, Cox DA, Turco M, Stuckey TD, Na Y, et al. (2005b): Prediction of mortality after primary percutaneous coronary intervention for acute myocardial infarction: the CADILLAC risk score. J Am Coll Cardiol 노, 1397-1405

Hamm C, Willems S: Koronare Herzerkrankung (KHK). In: Hamm C, Willems S (Hrsg.): Checkliste EKG. 4. Auflage; Thieme, Stuttgart u.a. 2014, 167-187

Hamm CW (2004a): Leitlinien: Akutes Koronarsyndrom (ACS) - Teil 1: ACS ohne persistierende ST-Hebung. Z Kardiol 93, 72-90

Hamm CW (2004b): Leitlinien: Akutes Koronarsyndrom (ACS) - Teil 2: ACS mit STHebung. Z Kardiol 93, 324-341

Hamm CW, Albrecht A, Bonzel T, Kelm M, Lange H, Schächinger V, Terres W, Voelker W (2008): Leitlinie: Diagnostische Herzkatheteruntersuchung. Clin Res Cardiol 97, 475512

Hanratty CG, Koyama Y, Rasmussen HH, Nelson GIC, Hansen PS, Ward MR (2002): Exaggeration of nonculprit stenosis severity during acute myocardial infarction: implications for immediate multivessel revascularization. J Am Coll Cardiol 40, 911-916

Henriques JPS, Hoebers LP, Råmunddal T, Laanmets P, Eriksen E, Bax M, Ioanes D, Suttorp MJ, Strauss BH, Barbato E, et al. (2016): Percutaneous Intervention for Concurrent Chronic Total Occlusions in Patients With STEMI. J Am Coll Cardiol 68, 1622-1632

Herold G: Innere Medizin 2015. Gerd Herold (Verlag), Köln 2014 
Hoebers LP, Vis MM, Claessen BE, van der Schaaf RJ, Kikkert WJ, Baan J, de Winter RJ, Piek JJ, Tijssen JGP, Dangas GD, Henriques JPS (2013): The impact of multivessel disease with and without a co-existing chronic total occlusion on short- and long-term mortality in ST-elevation myocardial infarction patients with and without cardiogenic shock. Eur J Heart Fail 15, 425-432

Hoebers LPC, Elias J, van Dongen IM, Ouweneel DM, Claessen BEPM, Piek JJ, Henriques JPS (2016): The impact of the location of a chronic total occlusion in a noninfarct-related artery on long-term mortality in ST-elevation myocardial infarction patients. EuroIntervention 12, 423-430

IQTIG - Institut für Qualitätssicherung und Transparenz im Gesundheitswesen. Qualitätsreport 2015. Berlin 2016

Irving J (2014): CTO pathophysiology: how does this affect management? Curr Cardiol Rev $10,99-107$

Ishihara M, Inoue I, Kawagoe T, Shimatani Y, Kurisu S, Hata T, Mitsuba N, Kisaka T, Nakama H, Kijima Y (2005): Comparison of the cardioprotective effect of prodromal angina pectoris and collateral circulation in patients with a first anterior wall acute myocardial infarction. Am J Cardiol 95, 622-625

Joyal D, Afilalo J, Rinfret S (2010): Effectiveness of recanalization of chronic total occlusions: a systematic review and meta-analysis. Am Heart J 160, 179-187

Katsuragawa M, Fujiwara H, Miyamae M, Sasayama S (1993): Histologic studies in percutaneous transluminal coronary angioplasty for chronic total occlusion: comparison of tapering and abrupt types of occlusion and short and long occluded segments. J Am Coll Cardiol 21, 604-611

Keeley EC, Boura JA, Grines CL (2003): Primary angioplasty versus intravenous thrombolytic therapy for acute myocardial infarction: a quantitative review of 23 randomised trials. Lancet $\underline{361}, 13-20$

Khan MF, Wendel CS, Thai HM, Movahed MR (2013): Effects of percutaneous revascularization of chronic total occlusions on clinical outcomes: a meta-analysis comparing successful versus failed percutaneous intervention for chronic total occlusion.

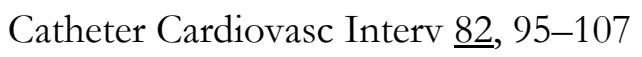

Killip T, Kimball JT (1967): Treatment of myocardial infarction in a coronary care unit. A two year experience with 250 patients. Am J Cardiol 20, 457-464 
Kirschbaum SW, Baks T, van den Ent M, Sianos G, Krestin GP, Serruys PW, de Feyter PJ, van Geuns R-JM (2008): Evaluation of left ventricular function three years after percutaneous recanalization of chronic total coronary occlusions. Am J Cardiol 101, 179_ 185

Kröger K, Libby P: Pathogenese, Prävention und Behandlung der Athersosklerose. In: Loscalzo J, Möckel M (Hrsg.): Harrisons Kardiologie. ABW Wissenschaftsverlag, Berlin 2011, 372-386

Kuno T, Numasawa Y, Miyata H, Takahashi T, Sueyoshi K, Ohki T, Negishi K, Kawamura A, Kohsaka S, Fukuda K (2013): Impact of coronary dominance on in-hospital outcomes after percutaneous coronary intervention in patients with acute coronary syndrome. PloS One $\underline{8}$, e 72672

Lapp H, Krakau I: Das Herzkatheterbuch: Diagnostische und interventionelle Kathetertechniken. 4. Auflage; Thieme, Stuttgart u.a. 2013

Leaman DM, Brower RW, Meester GT, Serruys P, van den Brand M (1981): Coronary artery atherosclerosis: severity of the disease, severity of angina pectoris and compromised left ventricular function. Circulation $\underline{63}, 285-299$

Lev EI, Kornowski R, Vaknin-Assa H, Porter A, Teplitsky I, Ben-Dor I, Brosh D, Fuchs S, Battler A, Assali A (2008): Comparison of the predictive value of four different risk scores for outcomes of patients with ST-elevation acute myocardial infarction undergoing primary percutaneous coronary intervention. Am J Cardiol 102, 6-11

Lexis CPH, van der Horst ICC, Rahel BM, Lexis MAS, Kampinga MA, Gu YL, de Smet BJGL, Zijlstra F (2011): Impact of chronic total occlusions on markers of reperfusion, infarct size, and long-term mortality: a substudy from the TAPAS-trial. Catheter Cardiovasc Interv 77, 484-491

Libby P (2002): Inflammation in atherosclerosis. Nature 420 , 868-874

Libby P, Theroux P (2005): Pathophysiology of coronary artery disease. Circulation 111, $3481-3488$

Mak KH, Moliterno DJ, Granger CB, Miller DP, White HD, Wilcox RG, Califf RM, Topol EJ (1997): Influence of diabetes mellitus on clinical outcome in the thrombolytic era of acute myocardial infarction. GUSTO-I Investigators. Global Utilization of Streptokinase and Tissue Plasminogen Activator for Occluded Coronary Arteries. J Am Coll Cardiol 30, $171-179$ 
Meier P, Hemingway H, Lansky AJ, Knapp G, Pitt B, Seiler C (2012): The impact of the coronary collateral circulation on mortality: a meta-analysis. Eur Heart J $\underline{33}, 614-621$

Mendis S, Thygesen K, Kuulasmaa K, Giampaoli S, Mähönen M, Ngu Blackett K, Lisheng L (2011): World Health Organization definition of myocardial infarction: 2008-09 revision. Int J Epidemiol 40, 139-146

Montalescot G, Sechtem U, Achenbach S, Andreotti F, Arden C, Budaj A, Bugiardini R, Crea F, Cuisset T, Di Mario C, et al. (2013): 2013 ESC guidelines on the management of stable coronary artery disease: the Task Force on the management of stable coronary artery disease of the European Society of Cardiology. Eur Heart J $\underline{34}$, 2949-3003

Moreno R, García E, Soriano J, Abeytua M, López de Sá E, Acosta J, Péerez de Isla L, Rubio R, López-Sendon JL (2001): Early coronary angioplasty for acute myocardial infarction: predictors of poor outcome in a non-selected population. J Invasive Cardiol $\underline{13}$, 202-210

Moreno R, Conde C, Perez-Vizcayno M-J, Villarreal S, Hernandez-Antolin R, Alfonso F, Bañuelos C, Angiolillo DJ, Escaned J, Fernandez-Ortiz A, Macaya C (2006): Prognostic impact of a chronic occlusion in a noninfarct vessel in patients with acute myocardial infarction and multivessel disease undergoing primary percutaneous coronary intervention. J Invasive Cardiol 18, 16-19

Morrow DA, Antman EM, Charlesworth A, Cairns R, Murphy SA, de Lemos JA, Giugliano RP, McCabe CH, Braunwald E (2000): TIMI risk score for ST-elevation myocardial infarction: A convenient, bedside, clinical score for risk assessment at presentation: An intravenous nPA for treatment of infarcting myocardium early II trial substudy. Circulation 102, 2031-2037

Mozid AM, Mohdnazri S, Mannakkara NN, Robinson NM, Jagathesan R, Sayer JW, Aggarwal RK, Clesham GJ, Gamma RA, Tang KH, et al. (2014): Impact of a chronic total occlusion in a non-infarct related artery on clinical outcomes following primary percutaneous intervention in acute ST-elevation myocardial infarction. J Invasive Cardiol $\underline{26}, 13-16$

Mukherjee D, Roffi M (2012): Chronic total occlusions in non-infarct-related arteries. Eur Heart J $\underline{33}, 695-697$

Neugebauer E, Bouillon B (1994): Was können Scoresysteme leisten? Unfallchirurg 97, $172-176$ 
Nienhuis MB, Ottervanger JP, Dambrink J-HE, de Boer M-J, Hoorntje JCA, Gosselink ATM, Suryapranata H, van 't Hof AWJ (2009): Comparative predictive value of infarct location, peak CK, and ejection fraction after primary PCI for ST elevation myocardial infarction. Coron Artery Dis 20, 9-14

Norris RM, Barnaby PF, Brandt PW, Geary GG, Whitlock RM, Wild CJ, Barratt-Boyes BG (1984): Prognosis after recovery from first acute myocardial infarction: determinants of reinfarction and sudden death. Am J Cardiol $\underline{53}, 408-413$

Nunn CM, O’Neill WW, Rothbaum D, Stone GW, O'Keefe J, Overlie P, Donohue B, Grines L, Browne KF, Vlietstra RE, et al. (1999): Long-term outcome after primary angioplasty: report from the primary angioplasty in myocardial infarction (PAMI-I) trial. J Am Coll Cardiol 33, 640-646

O’Connor SA, Garot P, Sanguineti F, Hoebers LP, Unterseeh T, Benamer H, Chevalier B, Hovasse T, Morice M-C, Lefèvre T, Louvard Y (2015): Meta-Analysis of the Impact on Mortality of Noninfarct-Related Artery Coronary Chronic Total Occlusion in Patients Presenting With ST-Segment Elevation Myocardial Infarction. Am J Cardiol 116, 8-14

Ohmann C, Gross-Weege W (1992): Scoring-Systeme auf der chirurgischen Intensivstation. I. Chirurg $\underline{63}, 1021-1028$

Ohmann C, Gross-Weege W (1993): Scoring-Systeme auf der chirurgischen Intensivstation. II. Chirurg $\underline{64}, 21-27$

Ohmann C, Lefering R (2002): Bedeutung von Scoringsystemen für die Chirurgie. Viszeralchirurgie $\underline{37}, 333-339$

Olivari Z, Rubartelli P, Piscione F, Ettori F, Fontanelli A, Salemme L, Giachero C, Di Mario C, Gabrielli G, Spedicato L, Bedogni F (2003): Immediate results and one-year clinical outcome after percutaneous coronary interventions in chronic total occlusions: data from a multicenter, prospective, observational study (TOAST-GISE). J Am Coll Cardiol $\underline{41}, 1672-1678$

O’Neill W, Timmis GC, Bourdillon PD, Lai P, Ganghadarhan V, Walton J, Ramos R, Laufer N, Gordon S, Schork MA (1986): A prospective randomized clinical trial of intracoronary streptokinase versus coronary angioplasty for acute myocardial infarction. $\mathrm{N}$ Engl J Med $\underline{314}, 812-818$

Pinger S: Repetitorium Kardiologie: Für Klinik, Praxis und Facharztprüfung. Deutscher Ärzte-Verlag, Köln 2010 
Rathore S, Matsuo H, Terashima M, Kinoshita Y, Kimura M, Tsuchikane E, Nasu K, Ehara M, Asakura Y, Katoh O, Suzuki T (2009): Procedural and in-hospital outcomes after percutaneous coronary intervention for chronic total occlusions of coronary arteries 2002 to 2008: impact of novel guidewire techniques. JACC Cardiovasc Interv 2, 489-497

Reimer KA, Jennings RB (1979): The „wavefront phenomenon“ of myocardial ischemic cell death. II. Transmural progression of necrosis within the framework of ischemic bed size (myocardium at risk) and collateral flow. Lab Invest $\underline{40}$, 633-644

Rentrop KP, Cohen M, Blanke H, Phillips RA (1985): Changes in collateral channel filling immediately after controlled coronary artery occlusion by an angioplasty balloon in human subjects. J Am Coll Cardiol $\underline{5}, 587-592$

Renz-Polster H, Krautzig S: Koronare Herzkrankheit. In: Renz-Polster H, Krautzig S (Hrsg.): Basislehrbuch Innere Medizin. 4. Auflage; Urban \& Fischer Verlag/Elsevier GmbH, München, Jena 2008, 63-66

Robert Koch-Institut (Hrsg.): Gesundheit in Deutschland. Gesundheitsberichterstattung des Bundes. Gemeinsam getragen von RKI und Destatis. Berlin 2015

Roffi M, Patrono C, Collet J-P, Mueller C, Valgimigli M, Andreotti F, Bax JJ, Borger MA, Brotons C, Chew DP, et al. (2016): 2015 ESC Guidelines for the management of acute coronary syndromes in patients presenting without persistent ST-segment elevation: Task Force for the Management of Acute Coronary Syndromes in Patients Presenting without Persistent ST-Segment Elevation of the European Society of Cardiology (ESC). Eur Heart $\mathrm{J} \underline{37}, 267-315$

Ross AM, Simoons LM, Lundergan C, Thompson M, Reiner J, Deychak Y, Rohrbeck S, Coyne K, Walker P, Cho S, et al. (1993): The effects of tissue plasminogen activator, streptokinase, or both on coronary-artery patency, ventricular function, and survival after acute myocardial infarction. The GUSTO Angiographic Investigators. N Engl J Med $\underline{329}$, $1615-1622$

Ryan TJ, Bauman WB, Kennedy JW, Kereiakes DJ, King SB, McCallister BD, Smith SC, Ullyot DJ (1993): Guidelines for percutaneous transluminal coronary angioplasty. A report of the American Heart Association/American College of Cardiology Task Force on Assessment of Diagnostic and Therapeutic Cardiovascular Procedures (Committee on Percutaneous Transluminal Coronary Angioplasty). Circulation 묘, 2987-3007

Sachdeva R, Uretsky BF (2011): The effect of CTO recanalization on FFR of the donor artery. Catheter Cardiovasc Interv Off J Soc Card Angiogr Interv 7, 367-369 
Sachdeva R, Agrawal M, Flynn SE, Werner GS, Uretsky BF (2013): Reversal of ischemia of donor artery myocardium after recanalization of a chronic total occlusion. Catheter Cardiovasc Interv Off J Soc Card Angiogr Interv 2, E453-458

Sachdeva R, Agrawal M, Flynn SE, Werner GS, Uretsky BF (2014): The myocardium supplied by a chronic total occlusion is a persistently ischemic zone. Catheter Cardiovasc Interv $\underline{83}, 9-16$

Schaper W (2009): Collateral circulation: past and present. Basic Res Cardiol 104, 5-21

Scholz KH, Maier SKG, Jung J, Fleischmann C, Werner GS, Olbrich HG, Ahlersmann D, Keating FK, Jacobshagen C, Moehlis H, et al. (2012): Reduction in treatment times through formalized data feedback: results from a prospective multicenter study of STsegment elevation myocardial infarction. JACC Cardiovasc Interv $\underline{5}, 848-857$

Şen Ö, Şen F, Topuz M, Allahverdiyev S, Baykan AO, Akkuş O, Sümbül Z, Çayli M (2016): Defining the prognosis of chronic total occlusions during primary percutaneous coronary intervention. Coron Artery Dis $\underline{27}, 207-212$

Shah PB (2011): Management of coronary chronic total occlusion. Circulation $\underline{123}, 1780$ 1784

Sianos G, Morel M-A, Kappetein AP, Morice M-C, Colombo A, Dawkins K, van den Brand M, Van Dyck N, Russell ME, Mohr FW, Serruys PW (2005): The SYNTAX Score: an angiographic tool grading the complexity of coronary artery disease. EuroIntervention $\underline{1}$, 219-227

Sianos G, Werner GS, Galassi AR, Papafaklis MI, Escaned J, Hildick-Smith D, Christiansen EH, Gershlick A, Carlino M, Karlas A, et al. (2012): Recanalisation of chronic total coronary occlusions: 2012 consensus document from the EuroCTO club. EuroIntervention $\underline{8}, 139-145$

Sorajja P, Gersh BJ, Cox DA, McLaughlin MG, Zimetbaum P, Costantini C, Stuckey T, Tcheng JE, Mehran R, Lansky AJ, et al. (2007): Impact of multivessel disease on reperfusion success and clinical outcomes in patients undergoing primary percutaneous coronary intervention for acute myocardial infarction. Eur Heart J $\underline{28}$, 1709-1716

Steg PG, James SK, Atar D, Badano LP, Blömstrom-Lundqvist C, Borger MA, Di Mario C, Dickstein K, Ducrocq G, Fernandez-Aviles F, et al. (2012): ESC Guidelines for the management of acute myocardial infarction in patients presenting with ST-segment elevation: The Task Force on the management of ST-segment elevation acute myocardial infarction of the European Society of Cardiology (ESC). Eur Heart J 33, 2569-2619 
Stone GW, Brodie BR, Griffin JJ, Grines L, Boura J, O’Neill WW, Grines CL (2000): Clinical and angiographic outcomes in patients with previous coronary artery bypass graft surgery treated with primary balloon angioplasty for acute myocardial infarction. Second Primary Angioplasty in Myocardial Infarction Trial (PAMI-2) Investigators. J Am Coll Cardiol $\underline{35}$, 605-611

Stone GW, Grines CL, Cox DA, Garcia E, Tcheng JE, Griffin JJ, Guagliumi G, Stuckey T, Turco M, Carroll JD, et al. (2002): Comparison of angioplasty with stenting, with or without abciximab, in acute myocardial infarction. N Engl J Med $\underline{346}$, 957-966

Stone GW, Kandzari DE, Mehran R, Colombo A, Schwartz RS, Bailey S, Moussa I, Teirstein PS, Dangas G, Baim DS, et al. (2005): Percutaneous recanalization of chronically occluded coronary arteries: a consensus document: part I. Circulation 112, 2364-2372

Stone PH, Raabe DS, Jaffe AS, Gustafson N, Muller JE, Turi ZG, Rutherford JD, Poole WK, Passamani E, Willerson JT (1988): Prognostic significance of location and type of myocardial infarction: independent adverse outcome associated with anterior location. J Am Coll Cardiol 11, 453-463

Suero JA, Marso SP, Jones PG, Laster SB, Huber KC, Giorgi LV, Johnson WL, Rutherford BD (2001): Procedural outcomes and long-term survival among patients undergoing percutaneous coronary intervention of a chronic total occlusion in native coronary arteries: a 20-year experience. J Am Coll Cardiol $\underline{38}$, 409-414

Suzuki S, Furui S, Kohtake H, Yokoyama N, Kozuma K, Yamamoto Y, Isshiki T (2006): Radiation exposure to patient's skin during percutaneous coronary intervention for various lesions, including chronic total occlusion. Circ J $\underline{70}, 44-48$

Tajstra M, Gasior M, Gierlotka M, Pres D, Hawranek M, Trzeciak P, Lekston A, Polonski L, Zembala M (2012): Comparison of five-year outcomes of patients with and without chronic total occlusion of noninfarct coronary artery after primary coronary intervention for ST-segment elevation acute myocardial infarction. Am J Cardiol 109, 208-213

Thompson CA, Jayne JE, Robb JF, Friedman BJ, Kaplan AV, Hettleman BD, Niles NW, Lombardi WL (2009): Retrograde techniques and the impact of operator volume on percutaneous intervention for coronary chronic total occlusions an early U.S. experience. JACC Cardiovasc Interv $\underline{2}, 834-842$ 
Thygesen K, Alpert JS, White HD, Jaffe AS, Apple FS, Galvani M, Katus HA, Newby LK, Ravkilde J, Chaitman B, et al. (2007): Universal definition of myocardial infarction: Kristian Thygesen, Joseph S. Alpert and Harvey D. White on behalf of the Joint ESC/ACCF/AHA/WHF Task Force for the Redefinition of Myocardial Infarction. Circulation 116, 2634-2653

Thygesen K, Alpert JS, Jaffe AS, Simoons ML, Chaitman BR, White HD, Katus HA, Apple FS, Lindahl B, Morrow DA, et al. (2012): Third universal definition of myocardial infarction. J Am Coll Cardiol 60, 1581-1598

Valenti R, Marrani M, Cantini G, Migliorini A, Carrabba N, Vergara R, Cerisano G, Parodi G, Antoniucci D (2014): Impact of chronic total occlusion revascularization in patients with acute myocardial infarction treated by primary percutaneous coronary intervention. Am J Cardiol 114, 1794-1800

Van der Schaaf RJ, Vis MM, Sjauw KD, Koch KT, Baan J, Tijssen JGP, de Winter RJ, Piek JJ, Henriques JPS (2006): Impact of multivessel coronary disease on long-term mortality in patients with ST-elevation myocardial infarction is due to the presence of a chronic total occlusion. Am J Cardiol 98, 1165-1169

Van der Schaaf RJ, Claessen BE, Vis MM, Hoebers LP, Koch KT, Baan J, Meuwissen M, Engstrom AE, Kikkert WJ, Tijssen JGP, et al. (2010): Effect of multivessel coronary disease with or without concurrent chronic total occlusion on one-year mortality in patients treated with primary percutaneous coronary intervention for cardiogenic shock. Am J Cardiol 105, 955-959

Veltman CE, van der Hoeven BL, Hoogslag GE, Boden H, Kharbanda RK, de Graaf MA, Delgado V, van Zwet EW, Schalij MJ, Bax JJ, Scholte AJHA (2015): Influence of coronary vessel dominance on short- and long-term outcome in patients after ST-segment elevation myocardial infarction. Eur Heart J $\underline{36}$, 1023-1030

Vermeer F, Oude Ophuis AJ, vd Berg EJ, Brunninkhuis LG, Werter CJ, Boehmer AG, Lousberg AH, Dassen WR, Bär FW (1999): Prospective randomised comparison between thrombolysis, rescue PTCA, and primary PTCA in patients with extensive myocardial infarction admitted to a hospital without PTCA facilities: a safety and feasibility study. Heart $\underline{\text { 82, }}$ 426-431

Wald DS, Morris JK, Wald NJ, Chase AJ, Edwards RJ, Hughes LO, Berry C, Oldroyd KG (2013): Randomized trial of preventive angioplasty in myocardial infarction. N Engl J Med $\underline{369}, 1115-1123$ 
Waldecker B, Waas W, Haberbosch W, Voss R, Tillmanns H (1997): Früh- und Langzeitergebnisse der direkten PTCA bei Patienten mit akutem Myokardinfarkt. Z Kardiol 무, 703-711

Weaver WD, Simes RJ, Betriu A, Grines CL, Zijlstra F, Garcia E, Grinfeld L, Gibbons RJ, Ribeiro EE, DeWood MA, Ribichini F (1997): Comparison of primary coronary angioplasty and intravenous thrombolytic therapy for acute myocardial infarction: a quantitative review. JAMA $\underline{278}, 2093-2098$

Werner GS (2014): The role of coronary collaterals in chronic total occlusions. Curr Cardiol Rev $\underline{10}, 57-64$

Werner GS, Ferrari M, Heinke S, Kuethe F, Surber R, Richartz BM, Figulla HR (2003): Angiographic assessment of collateral connections in comparison with invasively determined collateral function in chronic coronary occlusions. Circulation 107, 1972-1977

Werner GS, Surber R, Ferrari M, Fritzenwanger M, Figulla HR (2006): The functional reserve of collaterals supplying long-term chronic total coronary occlusions in patients without prior myocardial infarction. Eur Heart J 27, 2406-2412

Whitlow PL, Burke MN, Lombardi WL, Wyman RM, Moses JW, Brilakis ES, Heuser RR, Rihal CS, Lansky AJ, Thompson CA (2012): Use of a novel crossing and re-entry system in coronary chronic total occlusions that have failed standard crossing techniques: results of the FAST-CTOs (Facilitated Antegrade Steering Technique in Chronic Total Occlusions) trial. JACC Cardiovasc Interv $\underline{5}, 393-401$

WHO Consultation on Obesity (2000): Obesity: preventing and managing the global epidemic. Report of a WHO consultation. World Health Organ Tech Rep Ser $\underline{894}$, i-xii, 1 253

Widimský P, Budesínský T, Vorác D, Groch L, Zelízko M, Aschermann M, Branny M, St’ásek J, Formánek P (2003): Long distance transport for primary angioplasty vs immediate thrombolysis in acute myocardial infarction. Final results of the randomized national multicentre trial--PRAGUE-2. Eur Heart J 24, 94-104

Windecker S, Kolh P, Alfonso F, Collet J-P, Cremer J, Falk V, Filippatos G, Hamm C, Head SJ, Jüni P (2014): 2014 ESC/EACTS Guidelines on myocardial revascularization: The Task Force on Myocardial Revascularization of the European Society of Cardiology (ESC) and the European Association for Cardio-Thoracic Surgery (EACTS) Developed with the special contribution of the European Association of Percutaneous Cardiovascular Interventions (EAPCI). Eur Heart J 35, 2541-2619 
Wustmann K, Zbinden S, Windecker S, Meier B, Seiler C (2003): Is there functional collateral flow during vascular occlusion in angiographically normal coronary arteries? Circulation 107, 2213-2220

Yamamoto E, Natsuaki M, Morimoto T, Furukawa Y, Nakagawa Y, Ono K, Mitsudo K, Nobuyoshi M, Doi O, Tamura T, et al. (2013): Long-term outcomes after percutaneous coronary intervention for chronic total occlusion (from the CREDO-Kyoto registry cohort-2). Am J Cardiol 112, 767-774

Yang ZK, Zhang RY, Hu J, Zhang Q, Ding FH, Shen WF (2013): Impact of successful staged revascularization of a chronic total occlusion in the non-infarct-related artery on long-term outcome in patients with acute ST-segment elevation myocardial infarction. Int J Cardiol $\underline{165}, 76-79$

Zijlstra F, de Boer MJ, Hoorntje JC, Reiffers S, Reiber JH, Suryapranata H (1993): A comparison of immediate coronary angioplasty with intravenous streptokinase in acute myocardial infarction. N Engl J Med $\underline{328}$, 680-684

Zijlstra F, Hoorntje JC, de Boer MJ, Reiffers S, Miedema K, Ottervanger JP, van 't Hof AW, Suryapranata H (1999): Long-term benefit of primary angioplasty as compared with thrombolytic therapy for acute myocardial infarction. N Engl J Med $\underline{341}, 1413-1419$ 


\section{Danksagung}

Mein herzlicher Dank gilt ganz besonders Herrn Prof. Dr. Lars S. Maier für die Überlassung dieses spannenden und klinisch höchst relevanten Themas und für die außerordentliche Betreuung während der kompletten Promotionszeit. Nur durch seine Anregungen und Motivation konnte diese Arbeit vollendet werden.

Ich bedanke mich auch bei Frau Kristina Schröder und Frau Swetlana Hartmann aus der Studienambulanz der Klinik für Kardiologie und Pneumologie der Universitätsmedizin Göttingen für die großartige Mithilfe bei der Datenakquisition. Ohne ihren Einsatz wäre eine so vollständige Datenerfassung nicht möglich gewesen.

Dem ganzen Herzkatheterteam der Klinik für Kardiologie und Pneumologie der Universitätsmedizin Göttingen danke ich für die stets freundliche Hilfestellung bei der Einsicht in die benötigten koronarangiographischen Aufnahmen.

Herrn Achim Radtke danke ich ganz herzlich für die äußerst geduldige Unterstützung bei der Erstellung der Patientendatenbank und der Auswertung der erfassten Daten.

Ich danke zudem den Mitarbeitern des Instituts für Medizinische Statistik der Universitätsmedizin Göttingen (Direktor: Prof. Dr. T. Friede), die mich im Rahmen der Kurzberatung und des betreuten Arbeitens tatkräftig unterstützt haben. Ohne diese motivierte Betreuung wäre eine reibungslose statistische Datenauswertung nicht realisierbar gewesen. 